Modelo funcional heterocedástico com erro nas variáveis: uma abordagem para medidas repetidas

\author{
Alexandre Galvão Patriota \\ DISSERTAÇÃO APRESENTADA \\ $\mathrm{AO}$ \\ INSTITUTO DE MATEMÁTICA E ESTATÍSTICA \\ DA \\ UNIVERSIDADE DE SÃO PAULO \\ PARA \\ OBTENÇÃO DO GRAU DE MESTRE \\ EM \\ CIÊNCIAS. \\ Área de Concentração: Estatística \\ Orientador: Heleno Bolfarine
}

Durante a elaboração deste trabalho o autor recebeu apoio financeiro do $C N P q$

- São Paulo, Setembro de 2006 - 


\title{
Modelo Funcional Heterocedástico com Erro nas Variáveis uma Abordagem para Medidas Repetidas
}

\author{
Este exemplar corresponde à redação \\ final da dissertação devidamente corrigida e \\ defendida por Alexandre Galvão Patriota \\ e aprovada pela comissão julgadora.
}

São Paulo, Setembro de 2006

Comissão Julgadora:

- Prof. Dr. Heleno Bolfarine (Orientador) - IME/USP

- Prof. Dra. Denise Aparecida Botter - IME/USP

- Prof. Dr. Dorival Leão Pinto Júnior - ICMC/USP 


\section{AGRADECIMENTOS}

À minha mãe Leila Regina Patriota, pois foi a pessoa que mais me ajudou durante todos os anos da minha vida, me dando educação, apoio, incentivo e se dedicando em tempo integral para cuidar de todos os filhos. Eu me espelho na senhora e me espelharei durante toda a minha vida.

Ao meu irmão Marcelo e sua esposa Eulina pela companhia, conselhos e momentos de descontração. À minha vó Isolde, tia Lore e tia Rosana por toda a dedicação durante toda a minha adolescência. À minha vó Eny e meu vô Izaurino pelos sábios conselhos. Ao meu pai Edson pelos ensinamentos que carregarrei por toda a vida. Sinto muitas saudades de todos.

Ao meu grande professor João Maurício que sempre me ajudou a entender os teoremas de probabilidade e inferência. Agradeço pelo estímulo dado durante todos os anos. À professora Silvia Maria que me ajudou muito durante a graduação. Aos professores e funcionários do Departamento de Estatística e Matemática Aplicada que auxiliaram na minha formação.

Ao meu amigo Eric Gustavo pelas conversas e conselhos, pela amizade e companheirismo. Aos meus amigos Rafael Bráz (pela grande ajuda durante todo o mestrado e pela revisão da dissertação), Fabienne Rodriguez, Eveliny Barraso, Ênio Lopes, Chagas Almeida por todas as brincadeiras, conversas e dicas durante toda a graduação. Aos colegas Márcio, Juvêncio e Jaqueline pela hospedagem durante os primeiros seis meses do mestrado, Caio e Iesus pelas conversas, empréstimos de livros, discussões e risadas no período do mestrado. Aos camaradas Michel e Italo pelas conversas e momentos de descontração. Ao meu amigo Fernando Ferraz pela grande ajuda durante o mestrado. À Andreia Gouveia pela disposição e pela amizade. À todos os meus amigos de Curitiba, Fortaleza e São Paulo que de alguma forma contribuíram para esse momento.

Ao professor Heleno Bolfarine pelo apoio e orientação no mestrado. Aos professores Gilberto Avarenga, Mônica Sandoval, Nicolai Kolev, Antônio Carlos e Julia 
Pavan pelos cursos ministrados. À professora Denise Botter que me auxiliou na escolha do orientador. Ao professor Mário de Castro pela atenção. Aos professores Denise Botter e Dorival Leão por aceitarem participar da banca julgadora e pelas sugestões que foram fundamentais neste trabalho. Ao CNPq pelo auxílio financeiro. 


\section{SUMÁRIO}

Página

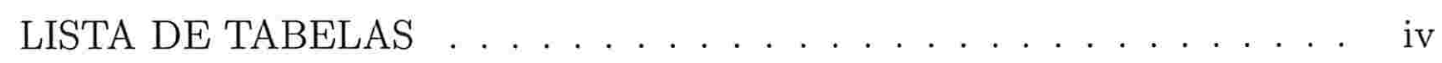

LISTA DE FIGURAS . . . . . . . . . . . . . . . . . vi vi

RESUMO ........................................

ABSTRACT ................. ix

1 Introdução 1

1.1 Formulação do problema . . . . . . . . . . . . . . . . 1

1.2 Apresentação dos capítulos . . . . . . . . . . . . . . . 3

1.3 Revisão da literatura . . . . . . . . . . . . . . . . . . . . . . . 4

2 Modelos com erro nas variáveis $\quad 8$

2.1 Estimação na presença de parâmetros de perturbação . . . . . . . . 8

2.2 Modelo funcional homocedástico com erro nas variáveis . . . . . . . . 10

2.3 Modelo funcional heterocedástico com erro nas variáveis . . . . . . 16

2.4 Propagação da incerteza dos métodos de medição . . . . . . . . . . 20

2.5 Modelo funcional para dados com réplicas . . . . . . . . . . . . 22

2.5.1 Repetições independentes . . . . . . . . . . . . 25

2.5.2 Repetições dependentes . . . . . . . . . . . . . . . 34

2.5.3 Extensão para comparação de várias retas . . . . . . . . . . 41

2.6 Resumo do capítulo . . . . . . . . . . . . . . . . . . . . . . 48 
3 Simulação $\quad 50$

3.1 Réplicas independentes . . . . . . . . . . . . . . . . . . . 51

3.2 Réplicas dependentes . . . . . . . . . . . . . . . . . . . . . . 79

4 Aplicação $\quad 90$

4.1 Análise de cobalto em jazidas . . . . . . . . . . . . . . . . 90

4.2 Análise descritiva . . . . . . . . . . . . . . . . . . . . . . . . . . . 94

4.3 Análise inferencial . . . . . . . . . . . . . . . . . . . . . . . . 100

5 Conclusões e comentários finais $\quad 103$

5.1 Conclusões . . . . . . . . . . . . . . . . . . . . . . . 103

5.2 Pesquisas futuras . . . . . . . . . . . . . . . . . 103

$\begin{array}{ll}\text { A Resultados } & 105\end{array}$

A.1 Dois métodos . . . . . . . . . . . . . . . . . . . . . 105

A.2 Vários métodos . . . . . . . . . . . . . . . . . . . . 116

$\begin{array}{lr}\text { Referências Bibliográficas } & 128\end{array}$ 


\section{LISTA DE TABELAS}

Página

2.1 Repetições feitas para a $i$-ésima concentração, para o modelo sem correlação . . . . . . . . . . . . . . . . . . . . 26

3.1 EQM e Viés estimados (concentração simétrica) . . . . . . . . . . . . 52

3.2 EQM e Viés estimados (concentração simétrica) . . . . . . . . . . . 53

3.3 Nível de significância estimado (concentração simétrica) . . . . . . . . 55

3.4 EQM e Viés estimados, usando o modelo com variâncias corrigidas (concentração simétrica), para $n=10,15 \ldots \ldots$. . . . . . 58

3.5 EQM e Viés estimados, usando o modelo com variâncias corrigidas (concentração simétrica), para $n=50 \ldots \ldots$. . . . . . . . 59

3.6 Nível de significância estimado, usando o modelo com variâncias corrigidas (concentração simétrica) . . . . . . . . . . . . . 61

3.7 Probabilidade de ocorrer o Erro tipo II nos Modelos 1 e 2, para $n=$ 10, 15, 50 e $r=15,25$ (concentração simétrica) . . . . . . . . . . 63

3.8 Probabilidade de ocorrer o Erro tipo II nos Modelos 1 e 2, para $n=$ $10,15,50$ e $r=50,100$ (concentração simétrica) . . . . . . . . 64

3.9 Probabilidade de ocorrer o Erro tipo II nos Modelos 1 e 2, para $n=$ $10,15,50$ e $r=150$ (concentração simétrica) . . . . . . . . 65

3.10 EQM e Viés estimados (concentração assimétrica) . . . . . . . . . . 69 
3.11 EQM e Viés estimados (concentração assimétrica) . . . . . . . . . . 70

3.12 Nível de significância estimado (concentração assimétrica) . . . . . . . 72

3.13 Probabilidade de ocorrer o Erro tipo II nos modelos 1 e 2, para $n=$ $10,15,50$ e $r=15,25$ (concentração assimétrica) . . . . . . . . 74

3.14 Probabilidade de ocorrer o Erro tipo II nos modelos 1 e 2, para $n=$ $10,15,50$ e $r=50,100$ (concentração assimétrica) . . . . . . . 75

3.15 Probabilidade de ocorrer o Erro tipo II nos modelos 1 e 2, para $n=$ $10,15,50$ e $r=150$ (concentração assimétrica) . . . . . . . . 76

3.16 EQM e Viés estimados para os Modelos 1, 2 e 3 (concentração simétrica) 80

3.17 EQM e Viés estimados para os Modelos 1, 2 e 3 (concentração simétrica) 81

3.18 Nível de significância estimado para os Modelos 1, 2 e 3 (concentração simétrica) . . . . . . . . . . . . . . . . . . 83

3.19 EQM e Viés estimados para os Modelos 1, 2 e 3 (concentração assimétrica) ........................... 85

3.20 EQM e Viés estimados para os Modelos 1, 2 e 3 (concentração assimétrica) . . . . . . . . . . . . . . . . . 86

3.21 Nível de significância estimado para os Modelos 1, 2 e 3 (concentração assimétrica) . . . . . . . . . . . . . . . . . . . . 88

4.1 Médias em cada log-concentração . . . . . . . . . . . . . . . . . . . . 94

4.2 Variâncias em cada log-concentração . . . . . . . . . . . . . . . 95

4.3 Estimativas (e erro-padrão) dos vieses para cada modelo . . . . . . . 101

4.4 Estimativas das variâncias e concentrações para cada modelo . . . . . 102 


\section{LISTA DE FIGURAS}

Página

2.1 Diagrama de dispersão de dois instrumentos de medição, para o caso homocedástico . . . . . . . . . . . . . . . . . . . 12

2.2 Diagrama de dispersão de dois instrumentos de medição, para o caso heterocedástico . . . . . . . . . . . . . . . . . . 17

2.3 Matriz de diagramas de dispersão de dois instrumentos de medição, para o caso em que existe repetições . . . . . . . . . . . . . . . . . 24

2.4 Gráfico das Médias . . . . . . . . . . . . . . . . . . . . . . 25

2.5 Gráfico do terreno dividido em dois blocos . . . . . . . . . . . . . . 32

3.1 Gráfico do viés dos parâmetros dos Modelos 1 e 2 (concentração simétrica) . . . . . . . . . . . . . . . . . . . . 54

3.2 Gráfico do nível de significância estimado dos modelos 1 e 2 (concentração simétrica) . . . . . . . . . . . . . . . . 56 56

3.3 Gráfico do viés dos parâmetros dos Modelos 1 e 2, usando o modelo com variância corrigida . . . . . . . . . . . . . . 60

3.4 Gráfico do nível de significância estimado dos modelos 1 e 2, usando modelo com variância corrigida (concentração simétrica) . . . . . . 62

3.5 Gráfico do poder do teste dos Modelos 1 e 2, usando modelo com variância corrigida . . . . . . . . . . . . . . 66 66 
3.6 Gráfico do poder do teste dos Modelos 1 e 2, usando modelo com variância corrigida . . . . . . . . . . . . . . 67

3.7 Gráfico do viés dos parâmetros dos Modelos 1 e 2, usando o modelo com variância corrigida (concentração assimétrica) . . . . . . . . . . . 71

3.8 Gráfico do nível de significância estimado dos modelos 1 e 2, usando modelo com variância corrigida (concentração assimétrica) . . . . . 73

3.9 Gráfico do poder do teste dos modelos 1 e 2, (concentração assimétrica) 77

3.10 Gráfico do poder do teste dos modelos 1 e 2, (concentração assimétrica) 78

3.11 Gráfico do viés dos parâmetros dos Modelos 1, 2 e 3 (concentração simétrica) . . . . . . . . . . . . . . . . . . 82

3.12 Gráfico do nível de significância estimado dos modelos 1, 2 e 3 (concentração simétrica) . . . . . . . . . . . . . . . . . . . . 84

3.13 Gráfico do viés dos parâmetros dos Modelos 1, 2 e 3 (concentração assimétrica $\ldots \ldots \ldots \ldots$. . . . . . . . . . . . . . . . . 87

3.14 Gráfico do nível de significância estimado dos modelos 1, 2 e 3 (concentração assimétrica $) \quad \ldots \ldots$. . . . . . . . . . . . . . . . . . . 89

4.1 Boxplot para cada concentração medida por PLAA . . . . . . . . . . 95

4.2 Boxplot para cada concentração medida por GQ6 . . . . . . . . . 96

4.3 Gráficos de Autocorrelações para PLAA . . . . . . . . . . . . . . 97

4.4 Gráficos de Autocorrelações parciais para PLAA . . . . . . . . . . 97

4.5 Gráficos de Autocorrelações para GQ6 . . . . . . . . . . . . . . . 98

4.6 Gráficos de Autocorrelações parciais para GQ6 . . . . . . . . . . . . 98

4.7 Gráficos das observações . . . . . . . . . . . . . . . . . . . . . . . . . 99

4.8 Gráfico das Médias . . . . . . . . . . . . . . . . . . . . . . . . 100 


\section{RESUMO}

Os modelos funcionais heterocedásticos com erro nas variáveis, quando não existem repetições, assumem que as variâncias são conhecidas. Na prática esta suposição nem sempre é satisfeita e repetições são feitas apenas para obter os valores das variâncias. Assim, depois de obtidas as variâncias amostrais, assume-se que as variâncias populacionais são conhecidas. Neste trabalho propomos um modelo que incorpora as repetições (correlacionadas ou não) no processo de estimação, considerando variâncias desconhecidas. As inferências propostas são baseadas na teoria de máxima verossimilhança (MV) e um algoritmo do tipo EM é utilizado para calcular as estimativas. A estatística de Wald é proposta para testar as hipóteses de interesse e um estudo de simulação é feito para descrever o comportamento dessa estatística em determinados tamanhos amostrais e parâmetros fixados a priori. A técnica proposta é aplicada na validação de métodos de medição, utilizando medidas da quantidade de cobalto feitas pela companhia Vale do Rio Doce. Os algoritmos foram implementados no programa estatístico R Development Core Team (2006). 


\begin{abstract}
The heteroscedastic functional error-in-variables model, when there are no replications, assumes known variances. In practice, this assumption is not always satisfied, and replicated measures are obtained in order to assess estimates for the variances. After the sample variances are obtained, it is assumed that the variances are known. In this work we propose a model which incorporates the repetitions (correlated or not) in the process of estimation, assuming unknown variances. The proposed inferential approaches are based on the maximum likelihood theory (ML) and an EM algorithm is used to compute the estimates. The Wald statistic is proposed to test the hypothesis of interest and a simulation study was carried out to describe the behavior of this statistic in certain sample sizes and a priori fixed parameters. The proposed approach is applied to the area of validation of methods of measurement, utilizing cobalt measures obtained by the Vale do Rio Doce company. The algorithms were implemented on the statistical environment $\mathrm{R}$ Development Core Team (2006).
\end{abstract}




\section{Capítulo 1}

\section{Introdução}

\subsection{Formulação do problema}

O problema prático que inspirou essa dissertação está presente em diversas áreas, como por exemplo, geologia, química, física, biologia, entre outras. De forma geral, o problema consiste em estimar os parâmetros de um modelo de regressão (heterocedástico) linear simples, quando a covariável não pode ser observada diretamente.

Estudar as relações entre: a quantidade de proteína na urina e a dosagem de uma determinada droga (Barnett, 1970), peso e o diâmetro de um tipo específico de concha do mar (Barnett, 1970), o rendimento na produção de um certo cereal e a verdadeira concentração de hidrogênio no solo (Fuller, 1987) e dois instrumentos de medição são problemas práticos onde a cováriavel é observada com erro de medida. E, como conseqüência, poderão ser tratados usando os modelos estudados nesta dissertação. Em particular, o problema de validação de métodos de medição é uma das aplicações. A validação de métodos tem o objetivo de comparar métodos de medição e concluir se um método pode ser considerado confiável ou não. Podemos definir medição como um conjunto de operações que se destina a mensurar algo de acordo com uma métrica definida previamente. Na prática a indústria (ou laboratório) utiliza 
um método, já consagrado (padrão), para medir a concentração de alguma substância. Assim, é natural surgir(em) um (vários) método(s) de medição, que tenha(m) algum atributo que não está presente no método (instrumento) consagrado. O método consagrado é o instrumento que mede sem tendência a concentração em questão, tendo geralmente altos custos, tempo de treinamento elevado para manipular o instrumento, mão de obra especializada e etc. Neste contexto, o interesse principal é testar a hipótese de que o(s) método(s) novo(s) não difere(m) de forma significativa do método consagrado. Duas características importantes deste problema, e que devem ser levadas em consideração, é que a variância depende da concentração, ou seja, para baixas concentrações espera-se variâncias pequenas e para altas concentrações variâncias grandes, e os métodos de medição mensuram com erro a concentração de interesse. As metodologias encontradas na literatura consideram um modelo funcional heterocedástico com erro nas variáveis supondo variâncias conhecidas, contudo, na prática, as variâncias não são conhecidas.

Testar a exatidão do(s) novo(s) método(s) é uma fase muito importante no processo de validação, afetando de forma significativa os custos de uma indústria de minério, por exemplo. No caso em que as variáveis externas não influenciam no processo de medição, repetições podem ser feitas com o objetivo de capturar a variabilidade do método utilizado. Neste tipo de problema as metodologias para validação de métodos disponíveis na literatura consideram que as variabilidades dos mesmos são conhecidas, e não incorporam no modelo a presença de medidas repetidas. Não é objetivo deste trabalho detalhar aspectos metrológicos, a validação de métodos servirá apenas como aplicação dos modelos que foram formulados.

Exemplos reais para o problema de validação de métodos podem ser encontrados em Castilho (2004) pg.278, 287, que descreve e compara alguns métodos para determinar a quantidade de ouro em um depósito de cobre-ouro. Riu e Rius (1996) comparam métodos para analisar a quantidade de sódio em amostras de águas, a resistência da densidade (em micrômetros) de várias películas, as concentrações do hidrocarboneto policíclico aromático, entre outros. Galea-Rojas et al. (2005) apre- 
sentam uma aplicação em uma mineradora de ferro.

Os objetivos gerais deste trabalho são: (1) revisar, descrever e comentar os modelos funcionais com erro nas variáveis, (2) propor extensões para os estimadores na presença de medidas repetidas, correlacionadas ou não e (3) aplicar os modelos estudados na validação de métodos.

Os objetivos específicos deste trabalho são: (1) comparar os modelos funcionais heterocedásticos com erro nas variáveis, usando duas abordagens diferentes na estimação das variâncias. As duas abordagens utilizam réplicas para estimar as variâncias, a primeira estima via método dos momentos (MM) e a segunda via máxima verossimilhança (MV). (2) Considerar, na estimação dos parâmetros, uma possível dependência das repetições. (3) Propor estimadores e testes, quando o interesse é comparar várias retas, que consideram a repetição dos dados. A comparação de vários métodos de medição com um método consagrado é uma das aplicações do item (3).

Reservaremos a palavra método(s) para identificar o(s) procedimento(s) que os laboratórios utilizam para mensurar as concentrações que se tem o interesse de comparar.

\subsection{Apresentação dos capítulos}

No Capítulo 2 descrevemos os modelos funcionais com erro nas variáveis, com ênfase na comparação de métodos de medição. No Capítulo 3 apresentamos algumas comparações dos procedimentos tratados no Capítulo 2 utilizando simulações. No Capítulo 4 utilizamos um conjunto de dados reais apenas para comparar as metodologias propostas. No Capítulo 5 apresentamos conclusões, comentários e possíveis extensões do trabalho realizado. E no apêndice A são apresentadas as demonstrações. 


\subsection{Revisão da literatura}

Os modelos clássicos de regressão consideram que as covariáveis do estudo são medidas sem erro. No entanto, é comum encontrar problemas onde as covariáveis são medidas com erro. Nestes casos a metodologia clássica de modelos de regressão não pode ser aplicada, pois algumas condições de regularidade não são satisfeitas, levando a estimadores não consistentes para os parâmetros do modelo.

A mais de um século, Adcock (1878) propôs o método dos mínimos quadrados ortogonais. Tal metodologia incorpora na estimação dos parâmetros o erro na medida da covariável. Para ilustrar a idéia de Adcock, suponha a relação $y_{i}=\alpha+\beta x_{i}$ para $i=1,2, \ldots, n$. No método dos mínimos quadrados ordinários (MQO) encontramos os valores de $\alpha$ e $\beta$ que minimizam $\sum\left(y_{i}-\alpha-\beta x_{i}\right)^{2}$. No caso em que a variável $x_{i}$ é medida com erro o estimador de MQ para $\beta$ não é consistente, ver Fuller (1987). No método dos mínimos quadrados ortogonais encontramos os valores de $\alpha$ e $\beta$ que minimizam $\frac{1}{1-\beta^{2}} \sum\left(y_{i}-\alpha-\beta x_{i}\right)^{2}$, corrigindo a inclinação $(\beta)$ e levando a estimadores consistentes para os parâmetros em questão (Fuller, 1987). Desde então, vários pesquisadores sugerem extensões para o modelo de Adcock que dependem das suposições feitas para as variáveis não observadas. Existem duas abordagens para tratar o problema de mensuração de covariáveis com erro, a primeira é conhecida como estrutural e considera uma distribuição para as covariáveis não observadas, a segunda é conhecida como funcional e trata as covariáveis não observadas como parâmetros incidentais. Ambas as abordagens trazem problemas teóricos, na primeira ocorre um problema de identificabilidade e na segunda ocorre o problema de verossimilhança ilimitada sendo necessário supor, em ambas as abordagens, que alguns parâmetros são conhecidos. Neste trabalho consideramos apenas o caso funcional por ser menos restritivo em relação a distribuição verdadeira das covariáveis não observadas, podendo a mesma ser assimétrica.

Barnett (1970) estudou o modelo funcional linear com medidas repetidas inde- 
pendentes, com a seguinte relação entre as variáveis observadas: $Y_{i j}=\alpha+\beta x_{i}+e_{i j} \mathrm{e}$ $X_{i j}=x_{i}+u_{i j}$, para $j=1, \ldots, n_{i}$ e os erros $e_{i j}$ e $u_{i j}$ não correlacionados, derivando estimadores de MV para $\alpha$ e $\beta$ e a respectiva matriz de covariâncias assintótica. O autor também comenta algumas aplicações nas áreas médica, biológica e geológica. Dolby e Lipton (1972) estudaram modelos não lineares com medidas repetidas independentes e erros correlacionados, neste artigo os autores consideram que $\operatorname{Corr}\left(e_{i j}, u_{i j}\right)=\rho_{i}$ e $\operatorname{Corr}\left(e_{i j}, u_{k l}\right)=0$ para $k \neq i$ ou $j \neq l$. Estes autores derivam os estimadores de máxima verossimilhança e a matriz de covariância para os parâmetros do modelo. Dolby et al. (1987) derivam estimadores de MV considerando o mesmo modelo de Barnett (1970), apenas flexibilizam as repetições para cada variável ( $X$ e $Y$ ), ou seja, $Y_{i j}$ para $j=1,2, \ldots, n_{i}$ e $X_{i l}$ para $l=1, \ldots, m_{i}$.

Os modelos, aditivos ou multiplicativos, homocedásticos ou heterocedásticos, de regressão múltipla ou polinomial com erro nas variáveis podem ser vistos em Fuller (1987). Uma revisão interessante sobre os modelos com erro nas variáveis é feita por Stefanski (2000), onde o autor também faz alguns comentários sobre as tendências das pesquisas nessa área.

No problema de validação de métodos, diversas abordagens foram propostas no decorrer dos últimos anos. No caso de comparações de dois métodos Barnett (1965) sugeriu utilizar o teste $t$-Student pareado. Westgard e Hunt (1973) fizeram uma comparação entre os testes $t$ pareado, $F$ e MQO (mínimos quadrados ordinários) perturbando os vieses. Os autores aconselham usar o MQO, dentre as metodologias comparadas, juntamente com técnicas gráficas para verificar se a relação linear entre os métodos é satisfeita.

Ripley e Thompson (1987) generalizam o modelo funcional com erro nas variáveis sugerido por Adcock (1878). Tal modelo considera o erro em ambos os métodos e leva em conta a heterocedasticidade dos dados quando a mesma é conhecida. Os autores ainda propuseram uma metodologia para comparar separadamente o intercepto e a inclinação, não levando em conta a covariância entre os estimadores obtidos. Adiante definiremos com mais clareza o modelo. Alguns procedimentos para detectar o 
viés são revisados em Riu e Rius (1996). Estes autores comparam três metodologias, a saber: MQO, mínimos quadrados ponderados (MQP) e mínimos quadrados bivariado (MQB). Na abordagem bivariada (MQB) é considerado que ambas as medidas são mensuradas com erro, e pode-se obter um intervalo de confiança conjunto para o intercepto e inclinação, apesar da matriz de covariâncias para os parâmetros não ser consistente, ver Lehmann (1998). Castilho (2004) faz uma revisão na literatura e compara via simulação de Monte Carlo as metodologias MQO, MQP, MQB, o teste de Wald, o modelo sugerido por Ripley e Thompson (1987) e alguns de seus casos particulares. A simulação é feita considerando 16 estruturas diferentes. O autor considera o modelo sem viés e com viés (de $5 \%$ ), coeficiente de variação de $8 \%$ e $0.8 \%$, distribuição uniforme e log-normal para a verdadeira concentração. O autor conclui que os modelos sugeridos por Ripley e Thompson (1987), York (1966), Williamson (1968) (York e Williamson sugerem mínimos quadrados usando a mesma ponderação que Ripley e Thompson 1987) e Riu e Rius (1996) produzem nível descritivo aceitável e poder bastante satisfatório.

Gimenez e Bolfarine (1997) derivam um estimador para a matriz de covariâncias no modelo sugerido por Ripley e Thompson (1987) obtido a partir da metodologia do escore corrigido, além disso os autores propuseram uma estatística Wald para testar o viés analítico conjunto (testar o intercepto e inclinação conjuntamente) com distribuição qui-quadrado assintótica. Galea-Rojas et al. (2003) consideram o modelo proposto por Ripley e Thompson (1987) e derivam estimadores consistentes para os parâmetros e para a matriz de covariâncias dos parâmetros a partir da logverossimilhança perfilada, uma estatística de Wald é proposta para testar o viés. Os autores concluem via simulação que o teste obtido pela estatística Wald produz nível de significância empírico próximo do nível de significância teórico, mesmo quando o tamanho da amostra é pequeno.

Em todas as propostas acima foi considerado apenas que dois métodos estavam sendo testados. Galea-Rojas et al. (2005) estendem o modelo proposto por Ripley e Thompson (1987), aumentando o número de novos métodos a serem validados para $r$ 
(excluindo o método antigo). Este modelo é ideal para verificar se vários laboratórios estão calibrados com um laboratório de referência. Os parâmetros são estimados via $\mathrm{MV}$, utilizando a verossimilhança perfilada. Os autores também derivam um estimador consistente para a matriz de covariância dos parâmetros e uma estatística Wald para testar o viés dos métodos em questão. Um estudo de simulação e uma aplicação a dados reais são feitas no artigo.

Castro et al. (2004) derivam estimadores consistentes para os parâmetros e sua matriz de covariâncias no modelo proposto por Ripley e Thompson (1987) e estendem os resultados para o caso de vários métodos, utilizando a metodologia da correção de Score. Os autores também propuseram uma estatística de Wald corrigida. 


\section{Capítulo 2}

\section{Modelos com erro nas variáveis}

Na primeira seção damos uma breve introdução à estimação na presença de parâmetros de perturbação; na segunda seção apresentamos o modelo funcional homocedástico com erro nas variáveis; na terceira seção deste capítulo descrevemos o modelo funcional heterocedástico com erro nas variáveis; na quarta seção propomos o modelo funcional heterocedástico com erro nas variáveis para dados com medidas repetidas em vários casos específicos (sem correlações e com correlação entre as repetições) e uma extensão desses modelos para o caso em que existem mais de dois métodos.

\subsection{Estimação na presença de parâmetros de per- turbação}

As seções deste capítulo tratam de modelos com parâmetros incidentais, ou seja, o número de parâmetros cresce conforme o tamanho da amostra cresce. Por este motivo apresentamos alguns resultados que podem ser vistos com mais detalhes em Mak (1982).

Sejam $\boldsymbol{Z}_{1}, \ldots, \boldsymbol{Z}_{n}$ vetores aleatórios (de dimensão $s$ ) independentes com função 
densidade $f_{i}\left(Z_{i}, \boldsymbol{\theta}, \lambda_{i}\right)$, sendo $\theta$ o vetor (de dimensão $s$ ) de parâmetros de interesse e $\lambda_{i}$ os parâmetros de perturbação. Assim, o logaritmo natural da verossimilhança é dado por

$$
\ell=\sum_{i=1}^{n} \log f_{i}\left(Z_{i}, \theta, \lambda_{i}\right)
$$

Uma forma de eliminar os parâmetros de perturbação da estimação é maximizar (2.1) em relação a $\lambda_{i}$ para todo $i=1, \ldots, n$ e $\boldsymbol{\theta}$ fixado. Desta forma, obtemos $\hat{\lambda}_{i}$ que maximiza (2.1) e é função apenas de $\boldsymbol{Z}_{i}$ e $\boldsymbol{\theta}$. As inferências sobre $\boldsymbol{\theta}$ são baseadas no logaritmo da verossimilhança perfilada, que não depende de $\lambda_{i}$, dado por

$$
\ell^{p}=\sum_{i=1}^{n} \log f_{i}\left(\boldsymbol{Z}_{i}, \boldsymbol{\theta}, \hat{\lambda}_{i}\right)=\sum_{i=1}^{n} g_{i}\left(\boldsymbol{Z}_{i}, \boldsymbol{\theta}\right)
$$

Assumindo que as duas primeiras derivadas de $g_{i}$ em relação a $\boldsymbol{\theta}$ existem, definimos as seguintes matrizes

$$
\boldsymbol{V}(\boldsymbol{\theta})=\frac{1}{n} \operatorname{Var}\left(\frac{\partial \ell^{p}}{\partial \boldsymbol{\theta}}\right) \quad \text { e } \quad \boldsymbol{A}(\boldsymbol{\theta})=\frac{1}{n} \mathrm{E}\left(\frac{\partial^{2} \ell^{p}}{\partial \boldsymbol{\theta} \partial \boldsymbol{\theta}^{\top}}\right) .
$$

Mak (1982) estudou algumas condições necessárias para que exista um $\hat{\theta}$ que maximiza $\ell^{p}$. É preciso assumir que

$$
\lim _{n \rightarrow \infty} \frac{1}{n} \sum_{i=1}^{n} \lambda_{i} \leq \infty \quad, \quad \lim _{n \rightarrow \infty} \frac{1}{n} \sum_{i=1}^{n}\left(\lambda_{i}-\bar{\lambda}\right)^{2} \leq \infty
$$

e para utilizar o teorema do limite central de Liapounov a condição abaixo é necessária

$$
\lim _{n \rightarrow \infty} \frac{1}{n^{1+\delta / 2}} \sum_{i=1}^{n}\left|\lambda_{i}\right|^{2+\delta}=0
$$

Se as condições acima estão satisfeitas, então Mak mostra que

$$
n^{1 / 2} \boldsymbol{V}(\boldsymbol{\theta})^{-1 / 2} \boldsymbol{A}(\boldsymbol{\theta})(\hat{\boldsymbol{\theta}}-\boldsymbol{\theta}) \stackrel{D}{\longrightarrow} N_{s}(\mathbf{0}, \boldsymbol{I})
$$

e por conseqüência, 


$$
n(\hat{\boldsymbol{\theta}}-\boldsymbol{\theta})^{\top} \boldsymbol{A}(\boldsymbol{\theta}) \boldsymbol{V}(\boldsymbol{\theta})^{-1} \boldsymbol{A}(\boldsymbol{\theta})(\hat{\boldsymbol{\theta}}-\boldsymbol{\theta}) \stackrel{D}{\longrightarrow} \chi^{2}(s)
$$

onde $\stackrel{D}{\longrightarrow}$ ' significa convergência em distribuição.

\subsection{Modelo funcional homocedástico com erro nas variáveis}

Existem dois enfoques em modelos com erro nas variáveis, o primeiro não supõe uma distribuição para a verdadeira concentração conhecido por modelo funcional, e o segundo considera uma distribuição para a verdadeira concentração conhecido por modelo estrutural. Neste trabalho consideramos apenas o modelo funcional por ser menos restritivo em relação a distribuição da concentração, podendo a mesma ser assimétrica. Essa abordagem gera alguns problemas na consistência dos estimadores pois algumas condições de regularidade não estão satisfeitas. Uma dessas condições é que o número de parâmetros cresce com o tamanho da amostra, ou seja, o modelo considera que as concentrações $x_{1}, \ldots, x_{n}$ são parâmetros incidentais. Considerando que os erros de medição seguem uma distribuição normal, o modelo funcional homocedástico é dado por

$$
Y_{i} \stackrel{i i d}{\sim} N\left(\alpha+\beta x_{i}, \lambda\right)
$$

$\mathrm{e}$

$$
X_{i} \stackrel{i i d}{\sim} N\left(x_{i}, \kappa\right)
$$

de modo que a distribuição conjunta de $\left(Y_{i}, X_{i}\right)$ é dada por

$$
\left(\begin{array}{c}
Y_{i} \\
X_{i}
\end{array}\right) \sim N_{2}\left(\left(\begin{array}{c}
\alpha+\beta x_{i} \\
x_{i}
\end{array}\right) ;\left[\begin{array}{cc}
\lambda & 0 \\
\cdot & \kappa
\end{array}\right]\right),
$$


para $i \in I=\{1,2, \ldots, n\}$, sendo

$Y_{i}$ : Valor observado da $i$-ésima variável dependente;

$X_{i}$ : Valor observado da $i$-ésima covariável;

$x_{i}$ : Valor verdadeiro da $i$-ésima covariável (não observado);

$\mathrm{Na}$ validação de métodos podemos interpretar $Y_{i}$ como o valor da $i$-ésima concentração observada pelo instrumento novo que pode possuir alguma vantagem em relação ao instrumento padrão, como por exemplo, custo mais baixo, maior facilidade na manipulação do equipamento (métodos, instrumentos) de medição, menor tempo de treinamento para que uma pessoa o opere, entre outros. A variável $X_{i}$ é o valor observado da $i$-ésima concentração medida pelo método padrão, o instrumento padrão mensura a concentração sem viés, mas com algum custo operacional não desejado. Os parâmetros $\alpha$ e $\beta$ são respectivamente o viés aditivo e multiplicativo, ou seja, eles indicam a tendência do método novo em relação ao de referência. O interesse principal é verificar se o método novo consegue mensurar sem viés a concentração desejada. A figura abaixo mostra o diagrama de dispersão entre dois instrumentos de medição quando as variâncias não dependem da concentração analisada. 
Figura 2.1: Diagrama de dispersão de dois instrumentos de medição, para o caso homocedástico

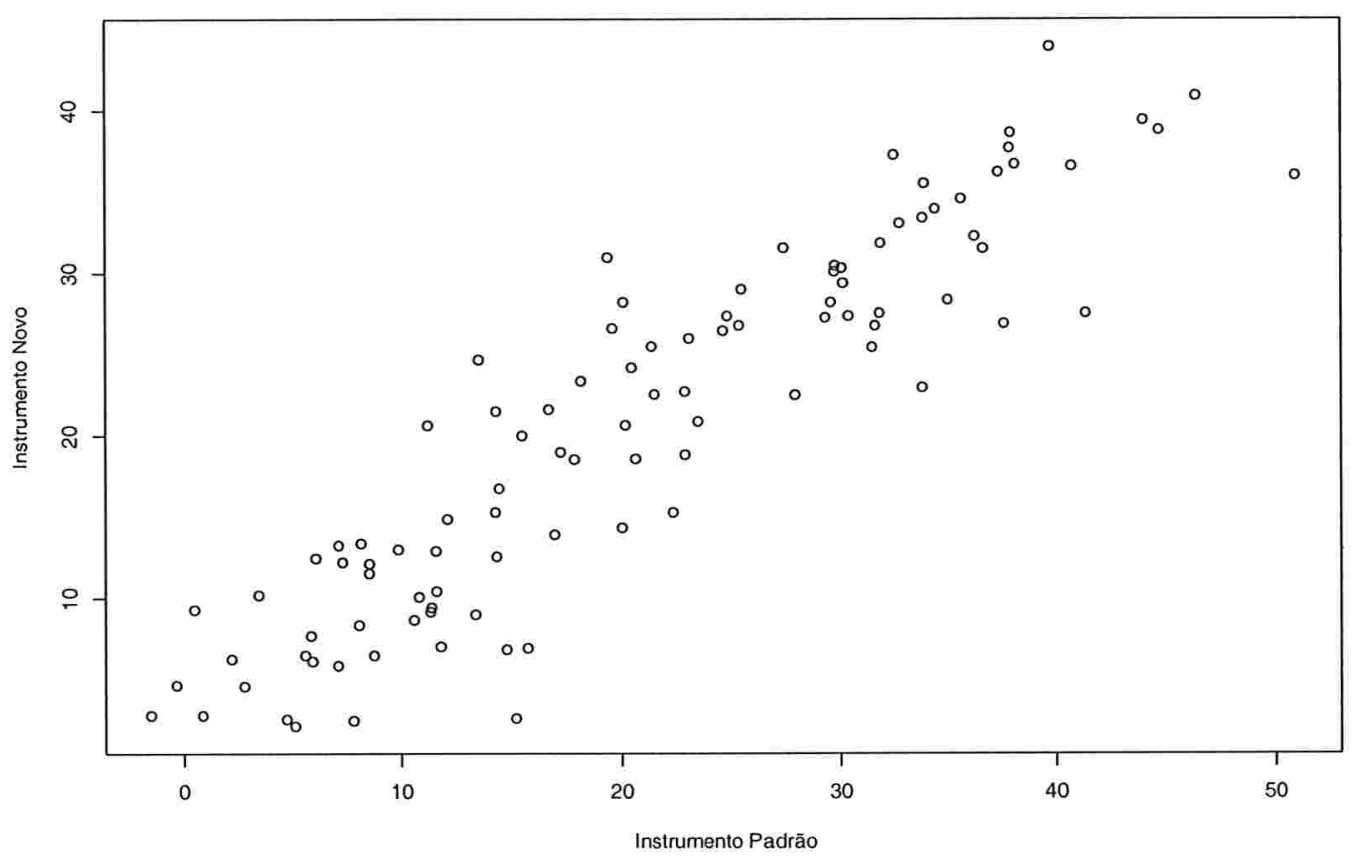

Os estimadores são derivados a partir da teoria da máxima verossimilhança, sendo assim o logaritmo da função de verossimilhança é dado por

$$
\ell(\theta)=\sum_{i \in I} \ell_{i}(\theta)
$$

sendo

$$
\ell_{i}(\theta)=-\log (2 \pi)-\frac{1}{2} \log (\kappa)-\frac{1}{2} \log (\lambda)-\frac{\left(y_{i}-\alpha-\beta x_{i}\right)^{2}}{2 \lambda}-\frac{\left(X_{i}-x_{i}\right)^{2}}{2 \kappa},
$$

e o parâmetro $\boldsymbol{\theta}=\left(\boldsymbol{\beta}^{\top}, \lambda, \kappa, \boldsymbol{x}^{\top}\right)^{\top}, \boldsymbol{\beta}=(\alpha, \beta)^{\top}$ e $\boldsymbol{x}=\left(x_{1}, \ldots, x_{n}\right)^{\top}$. Portanto, temos $n+4$ parâmetros para serem estimados. Uma das formas de obter estimadores consistentes para o viés aditivo $\alpha$ e para o viés multiplicativo $\beta$ é maximizar a logverossimilhança perfilada, ou seja, substituir em (2.3), $x_{i}$ pelo seu estimador de 
máxima verossimilhança (MV) dado por $\hat{x}_{i}$ obtendo

$$
\ell^{p}(\boldsymbol{\theta})=\sum_{i \in I} \ell_{i}^{p}(\boldsymbol{\theta})
$$

sendo

$$
\ell_{i}^{p}(\boldsymbol{\theta})=-\log (2 \pi)-\frac{1}{2} \log (\kappa)-\frac{1}{2} \log (\lambda)-\frac{\left(y_{i}-\alpha-\beta \hat{x}_{i}\right)^{2}}{2 \lambda}-\frac{\left(X_{i}-\hat{x}_{i}\right)^{2}}{2 \kappa}
$$

e $\hat{x}_{i}=\frac{\kappa \beta\left(Y_{i}-\alpha\right)+\lambda X_{i}}{\beta^{2} \kappa+\lambda}$. Além desse problema verifica-se que a verossimilhança acima é ilimitada, gerando problemas de maximização, quando $X_{i}=\hat{x}_{i}$ e $\kappa \rightarrow 0$. Uma das formas de contornar este problema é considerar conhecidos alguns parâmetros. Quando consideramos $\sigma=\lambda / \kappa$ conhecido, (substituindo $\lambda$ por $\sigma \kappa$ ) a log-verossimilhança se torna limitada, porém o estimador de MV para $\kappa$ não é consistente, ver Patefield (1978), um estimador consistente para $\kappa$ é dado por $2 \hat{\kappa}$ sendo que $\hat{\kappa}$ é o estimador de MV. Patefield (1978) mostra que os estimadores para $\alpha, \beta$ e $\kappa$ para o caso em que $\sigma$ é conhecido são dados por

$$
\begin{gathered}
\hat{\alpha}=\bar{Y}-\hat{\beta} \bar{X} \quad, \quad \hat{\beta}=\frac{S_{Y}^{2}-\sigma S_{X}^{2}+\sqrt{\left(S_{Y}^{2}-\sigma S_{X}^{2}\right)^{2}-4 \sigma S_{Y X}^{2}}}{2 S_{Y X}} \mathrm{e} \\
\hat{\kappa}=\sum_{i=1}^{n} \frac{\left(Y_{i}-\hat{\alpha}-\hat{\beta} X_{i}\right)^{2}}{n\left(\hat{\beta}^{2}+\sigma\right)},
\end{gathered}
$$

os quais serão consistentes se

$$
\lim _{n \rightarrow \infty} \sum_{i=1}^{n} x_{i} / n \rightarrow \mu_{*} \quad \text { e } \quad \lim _{n \rightarrow \infty} \sum_{i=1}^{n}\left(x_{i}-\mu_{*}\right)^{2} / n \rightarrow \nu_{*},
$$

sendo $\mu_{*}$ e $\nu_{*}$ constantes. Patefield (1977) deriva as variâncias assintóticas dos estimadores $\hat{\alpha}$ e $\hat{\beta}$ no caso ultraestrutural, ou seja, quando a verdadeira concentração $x_{i}$ tem distribuição normal com média $\mu_{x_{i}}$ e variância $\sigma_{x}^{2}$. Fazendo $\sigma_{x}^{2}=0$, temos como caso particular o modelo funcional. O estimador consistente para a matriz de covariâncias é dado abaixo

$$
\hat{\operatorname{Var}}(\hat{\boldsymbol{\beta}})=\frac{\left(\sigma+\hat{\beta}^{2}\right) \kappa \hat{\beta}}{n S_{Y X}}\left[\begin{array}{cc}
\bar{X}(1+\hat{\tau})+S_{Y X} / \hat{\beta} & -\bar{X}(1+\hat{\tau}) \\
-\bar{X}(1+\hat{\tau}) & (1+\hat{\tau})
\end{array}\right]
$$


sendo $\hat{\tau}=\frac{\sigma \hat{\kappa} \hat{\beta}}{\left(\sigma+\hat{\beta}^{2}\right) S_{Y X}}, S_{X Y}=1 / n \sum_{i=1}^{n}\left(X_{i}-\bar{X}\right) Y_{i}$ e $S_{X}^{2}=1 / n \sum_{i=1}^{n}\left(X_{i}-\bar{X}\right)^{2}$.

Assim, é possível utilizar a estatística de Wald para testar

$$
H_{0}:(\alpha, \beta)^{\top}=\left(\alpha_{0}, \beta_{0}\right)^{\top}
$$

contra $H_{1}:(\alpha, \beta)^{\top} \neq\left(\alpha_{0}, \beta_{0}\right)^{\top}$. A estatística Wald para este caso é dada por

$$
\xi_{W}=\frac{n S_{Y X}}{\left(\sigma+\hat{\beta}^{2}\right) \kappa \hat{\beta}}\left(\begin{array}{c}
\hat{\alpha}-\alpha_{0} \\
\hat{\beta}-\beta_{0}
\end{array}\right)^{\top}\left[\begin{array}{cc}
\bar{X}(1+\hat{\tau})+S_{Y X} / \hat{\beta} & -\bar{X}(1+\hat{\tau}) \\
-\bar{X}(1+\hat{\tau}) & (1+\hat{\tau})
\end{array}\right]^{-1}\left(\begin{array}{c}
\hat{\alpha}-\alpha_{0} \\
\hat{\beta}-\beta_{0}
\end{array}\right)
$$

Se $\alpha_{0}=0$ e $\beta_{0}=1$ o teste acima é equivalente, na validação de métodos, a testar se não existe viés aditivo $(\alpha=0)$ e multiplicativo $(\beta=1)$ na medição do instrumento novo.

Pelas propriedades dos estimadores obtidos maximizando a log-verossimilhança perfilada, ver Mak (1982), pode-se mostrar que a distribuição assintótica de $\xi_{W}$ é quiquadrado com 2 graus de liberdade. Outro procedimento pode ser adotado para contornar o problema de verossimilhança ilimitada. Podemos considerar $\kappa$ conhecido, o que torna a log-verossimilhança perfilada limitada. No entanto o estimador de MV para $\beta$ não é consistente, ver Fuller (1987), o que leva ao uso do estimador de MV para o caso estrutural é usado para estimar $\beta$, ver Cheng e Van Ness (1991). Os estimadores consistentes para $\alpha, \beta$ e $\lambda$, quando $\kappa$ é conhecido, são dados por

$$
\begin{gathered}
\hat{\alpha}=\bar{Y}-\hat{\beta} \bar{X} \quad, \quad \hat{\beta}_{e}=\frac{S_{X Y}}{S_{X}^{2}-\kappa} \mathrm{e} \\
\hat{\lambda}=S_{Y}^{2}-\hat{\beta} S_{Y X} .
\end{gathered}
$$

Cheng e Van Ness (1991) encontram a matriz de covariâncias assintótica de $\hat{\boldsymbol{\beta}}$ que é dada por 


$$
\hat{\operatorname{Var}}(\hat{\boldsymbol{\beta}})=1 / n\left[\begin{array}{cc}
\hat{\lambda}+\hat{\beta}^{2} \kappa+\bar{X}^{2} \hat{\psi} & -\bar{X} \hat{\psi} \\
-\bar{X} \hat{\psi} & \hat{\psi}
\end{array}\right]
$$

sendo $\hat{\psi}=\frac{\left\{\left(\hat{\nu}_{*}+\kappa\right)\left(\hat{\lambda}+\hat{\beta}^{2} \kappa\right)+\hat{\beta}^{2} \kappa^{2}\right\}}{\hat{\nu}_{*}^{2}}$ e $\hat{\nu}_{*}=S_{X}^{2}-\kappa$. Desta forma para testar os parâmetros do modelo podemos utilizar a estatística de Wald de forma análoga ao caso em que $\sigma=\lambda / \kappa$ é conhecido. A estatística é dada por

$$
\xi_{W}=n\left(\begin{array}{c}
\hat{\alpha}-\alpha_{0} \\
\hat{\beta}-\beta_{0}
\end{array}\right)^{\top}\left[\begin{array}{cc}
\hat{\lambda}+\hat{\beta}^{2} \kappa+\bar{X}^{2} \hat{\psi} & -\bar{X} \hat{\psi} \\
-\bar{X} \hat{\psi} & \hat{\psi}
\end{array}\right]^{-1}\left(\begin{array}{c}
\hat{\alpha}-\alpha_{0} \\
\hat{\beta}-\beta_{0}
\end{array}\right)
$$

Podemos também considerar $\lambda$ e $\kappa$ conhecidos, assim os parâmetros $\alpha$ e $\beta$ são estimados consistentemente, fazendo $\sigma=\lambda / \kappa$. Os estimadores encontrados usando a verossimilhança perfilada dos parâmetros são dados por

$$
\hat{\alpha}=\bar{Y}-\hat{\beta} \bar{X} \quad, \quad \hat{\beta}=\frac{S_{Y}^{2}-\sigma S_{X}^{2}+\sqrt{\left(S_{Y}^{2}-\sigma S_{X}^{2}\right)^{2}-4 \sigma S_{Y X}^{2}}}{2 S_{Y X}}
$$

respectivamente, que coincidem com o caso em que apenas $\sigma$ é conhecido. A matriz de covariâncias assintótica de $\hat{\boldsymbol{\beta}}$ no caso em que ambas as variâncias são conhecidas é

$$
\hat{\operatorname{Var}}(\hat{\boldsymbol{\beta}})=1 / n\left[\begin{array}{cc}
\lambda+\hat{\beta}^{2} \kappa+\bar{X}^{2} \hat{\psi} & -\bar{X} \hat{\psi} \\
-\bar{X} \hat{\psi} & \hat{\psi}
\end{array}\right]
$$

sendo $\hat{\psi}=\frac{\left\{\left(\hat{\nu}_{*}+\kappa\right)\left(\lambda+\hat{\beta}^{2} \kappa\right)+\hat{\beta}^{2} \kappa^{2}\right\}}{\hat{\nu}_{*}^{2}}$ e $\hat{\nu}_{*}=S_{X}^{2}-\kappa$. A estatística Wald para testar a hipótese formulada em (2.6) é dada abaixo

$$
\xi_{W}=n\left(\begin{array}{c}
\hat{\alpha}-\alpha_{0} \\
\hat{\beta}-\beta_{0}
\end{array}\right)^{\top}\left[\begin{array}{cc}
\lambda+\hat{\beta}^{2} \kappa+\bar{X}^{2} \hat{\psi} & -\bar{X} \hat{\psi} \\
-\bar{X} \hat{\psi} & \hat{\psi}
\end{array}\right]^{-1}\left(\begin{array}{c}
\hat{\alpha}-\alpha_{0} \\
\hat{\beta}-\beta_{0}
\end{array}\right) .
$$

De acordo com as propriedades dos estimadores obtidos maximizando a verossimilhança perfilada, estudadas por Mak em 1982, a distribuição das estatísticas acima é quiquadrado com 2 graus de liberdade. 


\subsection{Modelo funcional heterocedástico com erro nas variáveis}

A suposição de homocedasticidade não é satisfeita em diversos problemas reais. Por exemplo, em medidas de concentrações, áreas, pesos e diâmetros espera-se que a variância cresça junto com a média. Na validação de instrumentos de medição, as variâncias dependem da verdadeira concentração não observada. Para que um método de medição seja considerado confiável, é natural testa-lo em várias concentrações diferentes (baixas, médias e altas). A variedade das concentrações ocasiona a heterocedasticidade dos dados observados em ambos os instrumentos.

Barnett (1970) discutiu a aplicação de modelos funcionais heterocedásticos na avaliação da quantidade de proteína na urina em função da dosagem de uma determinada droga. Ripley e Thompson (1987), Riu e Rius (1996), Gimenez e Bolfarine (1997), Galea-Rojas et al. (2003), Castro et al. (2004), Castilho (2004), Galea-Rojas et al. (2005) e Castro et al. (2005) aplicam o modelo heterocedástico na validação de métodos de medição.

A Figura 2.2 descreve um possível comportamento de dois instrumentos de medição. As observações do gráfico foram simuladas supondo que o instrumento novo mede sem tendência. 
Figura 2.2: Diagrama de dispersão de dois instrumentos de medição, para o caso heterocedástico

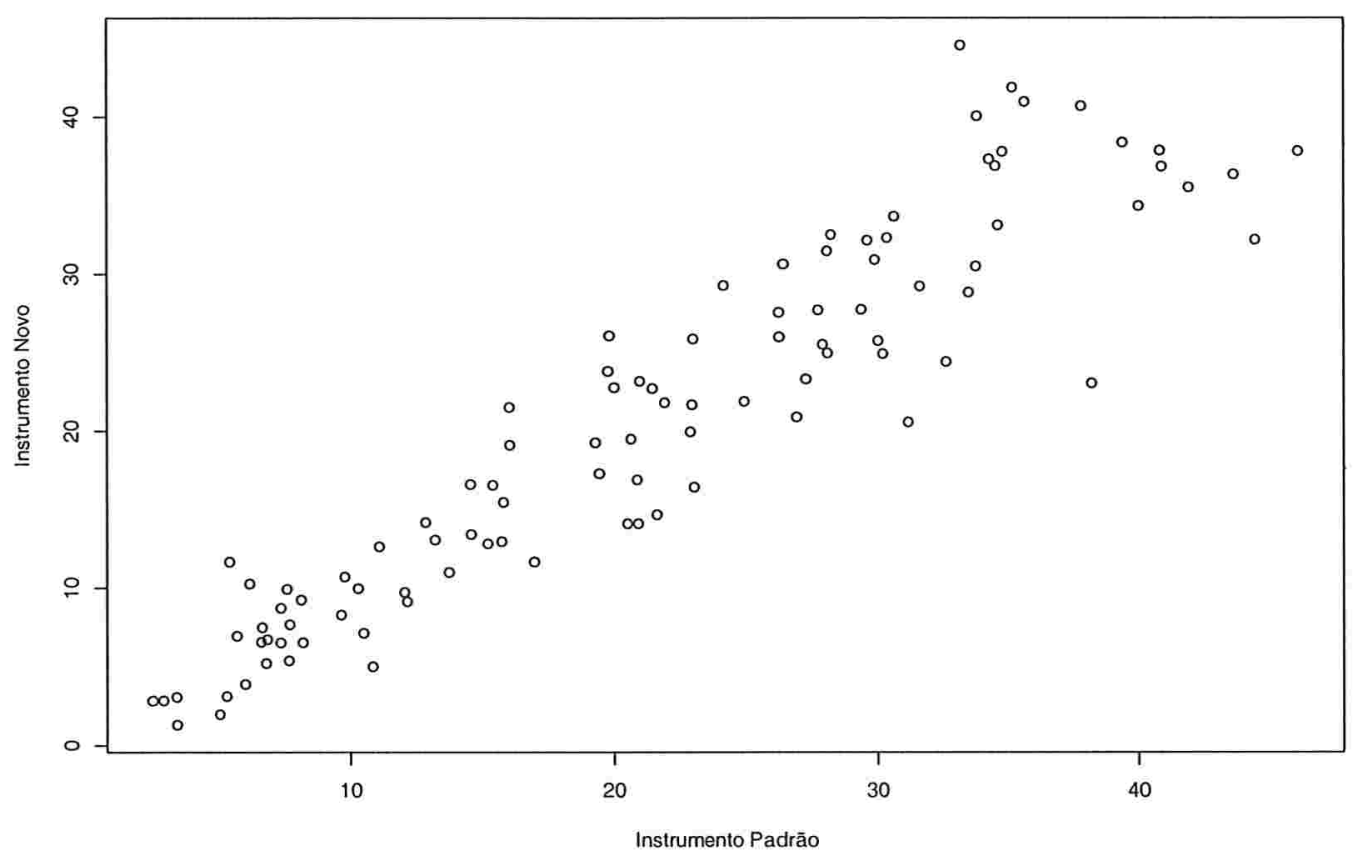

A Figura 2.2 descreve bem o comportamento heterocedástico de ambos os instrumentos. Percebe-se que quando a concentração aumenta a variabilidade aumenta. Nesta seção estudaremos o modelo funcional heterocedástico com erro nas variáveis, descrevendo as principais características do modelo e quais suposições são necessárias para utilizar tal modelo. A distribuição conjunta de $\left(Y_{i}, X_{i}\right)$, supondo normalidade dos erros, é dada por

$$
\left(\begin{array}{c}
Y_{i} \\
X_{i}
\end{array}\right) \sim N_{2}\left(\left(\begin{array}{c}
\alpha+\beta x_{i} \\
x_{i}
\end{array}\right) ;\left[\begin{array}{cc}
\lambda_{i} & 0 \\
\cdot & \kappa_{i}
\end{array}\right]\right)
$$

as variáveis $Y_{i}$ e $X_{i}$ e os parâmetros $\alpha$ e $\beta$ têm a mesma interpretação que no caso homocedástico, $\lambda_{i}$ e $\kappa_{i}$ são variâncias conhecidas de $Y_{i}$ e $X_{i}$ para $i=1, \ldots, n$ respec- 
tivamente. Assim, o logaritmo da função de verossimilhança é dada por

$$
\ell(\theta)=\sum_{i \in I} \ell_{i}(\theta)
$$

sendo

$$
\ell_{i}(\theta)=-\log (2 \pi)-\frac{1}{2} \log \left(\kappa_{i}\right)-\frac{1}{2} \log \left(\lambda_{i}\right)-\frac{\left(y_{i}-\alpha-\beta x_{i}\right)^{2}}{2 \lambda_{i}}-\frac{\left(X_{i}-x_{i}\right)^{2}}{2 \kappa_{i}},
$$

e o vetor de parâmetros $\boldsymbol{\theta}=(\boldsymbol{\beta}, \boldsymbol{x})^{\top}, \boldsymbol{\beta}=(\alpha, \beta)^{\top}$ e $\boldsymbol{x}=\left(x_{1}, \ldots, x_{n}\right)^{\top}$. A logverossimilhança (2.8) é limitada, pois as variâncias são conhecidas. Ripley e Thompson (1987) consideram o modelo heterocedástico acima e derivam os estimadores para $\alpha$ e $\beta$, a partir da verossimilhança perfilada que se obtém substituindo $x_{i}$ por $\hat{x}_{i}=\frac{\kappa_{i} \beta\left(Y_{i}-\alpha\right)+\lambda_{i} X_{i}}{\beta^{2} \kappa_{i}+\lambda_{i}}$, pois $\hat{x}_{i}$ maximiza (2.8) em relação a $x_{i}$. Os autores também propuseram testes marginais para $\alpha$ e $\beta$, sendo que as variâncias usadas para o teste são obtidas por analogia a metodologia de mínimos quadrados, que não são consistentes. Galea-Rojas et al. (2003) encontram estimativas consistentes para a matriz de covariâncias de $\hat{\alpha}$ e $\hat{\beta}$ baseadas na teoria de estimação para parâmetros incidentais usando a verossimilhança perfilada, ver Mak (1982). Estes autores demonstram que os estimadores para $\alpha$ e $\beta$ são

$$
\hat{\beta}=\frac{\sum_{i \in I} \omega_{i} \hat{x}_{i}\left(Y_{i}-\bar{Y}_{\omega}\right)}{\sum_{i \in I} \omega_{i} \hat{x}_{i}\left(X_{i}-\bar{X}_{\omega}\right)}, \hat{\alpha}=\bar{Y}_{\omega}-\hat{\beta} \bar{X}_{\omega}
$$

sendo que

$$
\bar{Y}_{\omega}=\sum_{i \in I} \frac{\omega_{i} \bar{Y}_{i}}{\sum \omega_{i}}, \bar{X}_{\omega}=\sum_{i \in I} \frac{\omega_{i} \hat{X}_{i}}{\sum \omega_{i}}
$$

$\mathrm{e}$

$$
\omega_{i}=\frac{1}{\lambda_{i}+\beta^{2} \kappa_{i}}, i=\{1,2, \ldots, n\}
$$

Para obter as estimativas de máxima verossimilhança faz-se necessário um método iterativo. O algoritmo proposto pelos autores segue os seguintes passos: (1) 
Inicia-se o procedimento iterativo com $v=0$ e os valores iniciais $\alpha^{(0)}=0, \beta^{(0)}=1$, (2) calcule $\hat{x}_{i}^{(v)},(3)$ calcule $\hat{\beta}^{(v)}$ e $\hat{\alpha}^{(v)}$, (4) incremente uma unidade a $v$, (5) repita os passos 2, 3 e 4 até a convergência. A matriz de covariâncias assintótica para os estimadores é obtida calculando

$$
\operatorname{Var}(\hat{\boldsymbol{\beta}})=(1 / n) W^{-1} V W^{-1}=\left[\begin{array}{cc}
\sigma_{\alpha \alpha} & \sigma_{\alpha \beta} \\
\sigma_{\beta \alpha} & \sigma_{\beta \beta}
\end{array}\right]
$$

sendo que

$$
W=(1 / n) \sum_{i=1}^{n} E\left\{\frac{\partial^{2} \ell^{p}(\boldsymbol{\theta})}{\partial \boldsymbol{\beta}^{2}}\right\} \quad \text { e } \quad V=(1 / n) \sum_{i=1}^{n} E\left\{\left(\frac{\partial \ell^{p}(\boldsymbol{\theta})}{\partial \boldsymbol{\beta}}\right)\left(\frac{\partial \ell^{p}(\boldsymbol{\theta})}{\partial \boldsymbol{\beta}}\right)^{\top}\right\}
$$

Galea-Rojas et al. (2003) mostram que os elementos da matriz de covariâncias assintótica de $\hat{\boldsymbol{\beta}}$ são

$$
\begin{gathered}
\sigma_{\alpha \alpha}=\frac{1}{\sum \omega_{i}}+\frac{\bar{x}_{\omega}^{2}}{S S_{\omega}}+n k\left(\frac{\bar{x}_{\omega}}{S S_{\omega}}\right)^{2}, \\
\sigma_{\alpha \beta}=\sigma_{\beta \alpha}=-\frac{\bar{x}_{\omega}}{S S_{\omega}}\left(1+\frac{n k}{S S_{\omega}}\right) \mathrm{e} \\
\sigma_{\beta \beta}=\frac{1}{S S_{\omega}}\left(1+\frac{n k}{S S_{\omega}}\right)
\end{gathered}
$$

sendo que

$$
S S_{\omega}=\sum \omega_{i}\left(x_{i}-\bar{x}_{\omega}\right)^{2} \quad, \quad \bar{x}_{\omega}=\frac{\sum \omega_{i} x_{i}}{\sum \omega_{i}} \quad \text { e } \quad k=1 / n \sum \omega_{i}^{2} \lambda_{i} \kappa_{i} .
$$

Para estimar $\operatorname{Var}(\hat{\boldsymbol{\beta}})$ consistentemente, basta substituir $\alpha, \beta, x_{i}$ e $x_{i}^{2}$ por $\hat{\alpha}, \hat{\beta}$, $\hat{x}_{i}$ e $\left(\hat{x}_{i}^{2}-\lambda_{i} \kappa_{i} \hat{\omega}_{i}\right)$, respectivamente que são as estimativas obtidas no último passo do processo iterativo. O estimador para $\omega_{i}$ é dado por $\hat{\omega}_{i}=\frac{1}{\lambda_{i}+\hat{\beta}^{2} \kappa_{i}}$. De forma análoga ao caso homocedástico, é possível usar a estatística de Wald para testar os parâmetros de interesse 


$$
\xi_{W}=\left(\begin{array}{c}
\hat{\alpha}-\alpha_{0} \\
\hat{\beta}-\beta_{0}
\end{array}\right)^{\top}\left[\begin{array}{cc}
\sigma_{\alpha \alpha} & \sigma_{\alpha \beta} \\
\sigma_{\beta \alpha} & \sigma_{\beta \beta}
\end{array}\right]^{-1}\left(\begin{array}{c}
\hat{\alpha}-\alpha_{0} \\
\hat{\beta}-\beta_{0}
\end{array}\right) \stackrel{a}{\sim} \chi^{2}(2)
$$

Galea-Rojas et al. (2003) concluíram através de um estudo de simulação que a estatística (2.9) é confiável mesmo quando o tamanho de amostra é pequeno. Esse resultado é válido apenas quando as variâncias são conhecidas. No Capítulo 3 faremos um estudo de simulação para descrever o comportamento dos estimadores e da estatística (2.9), quando as variâncias não são conhecidas e são estimadas usando réplicas.

Os modelos acima podem ser estendidos para o caso em que existem vários métodos a serem validados. Castro et al. (2005) derivaram os estimadores para este caso, usando a verossimilhança perfilada, e propuseram uma estatística de Wald para testar a tendência dos métodos. E, no apêncice do artigo, uma revisão sobre estimação na presença de parâmetros de perturbação é feita.

\subsection{Propagação da incerteza dos métodos de me- dição}

Quando estamos fazendo referência a métodos de medição, nós usamos as letras $X$ e $Y$ para identificar o instrumento padrão e o instrumento que temos interesse em validar respectivamente. Em algumas situações as variáveis $X$ e $Y$ não são observadas diretamente, mas são determinadas através de outras $p$ variáveis. Por exemplo, para a $i$-ésima medição teremos

$$
Y_{i}=\sum_{j=1}^{p_{1}} c_{1 i j} W_{1 i j}=c_{1 i}^{\top} \boldsymbol{w}_{1 i} \quad \text { e } \quad X_{i}=\sum_{j=1}^{p_{2}} c_{2 i j} W_{2 i j}=\boldsymbol{c}_{2 i}^{\top} \boldsymbol{w}_{2 i}
$$

sendo que $W_{1 i j}$ e $W_{2 i j}$ são variáveis de entrada observadas com distribuições $F_{W_{1 i j}}\left(w_{1}\right)$ e $F_{W_{2 i j}}\left(w_{2}\right)$ respectivamente. Dessa forma o teorema do limite central garante que, se forem somas de variáveis independentes com os quatro primeiros momentos finitos, 
$Y_{i}$ e $X_{i}$ têm distribuição normal. Quando $p_{1}=p_{2}=1$ as variáveis $X_{i j}$ e $Y_{i j}$ são observadas diretamente.

Na prática, assume-se que as variâncias de $W_{1 i j}$ e $W_{2 i j}$ são conhecidas para todo o $i$ e $j$. Assim, as variâncias de $Y_{i}$ e $X_{i}$ são calculadas da seguinte forma

$$
\lambda_{i}=\sum_{j=1}^{p_{1}} c_{1 i}^{2} \operatorname{Var}\left(W_{1 i j}\right) \quad \text { e } \quad \kappa_{i}=\sum_{j=1}^{p_{2}} c_{2 i}^{2} \operatorname{Var}\left(W_{2 i j}\right)
$$

e o modelo da Seção 2.7 pode ser usado. Se a distribuição de $W_{k i j}$, para $k=1,2$ for mal especificada, então as variâncias $\lambda_{i}$ e $\kappa_{i}$ podem ser subestimadas ou sobre estimadas. Acreditamos que, quando as variâncias são subestimadas, a estatística (2.9) rejeitará mais vezes a hipótese de validação do método novo do que o esperado. E quando as variâncias são sobre estimadas, a tendência é que estatística do teste seja mais conservadora, ou seja, aceite que as medidas do método novo seja confiável mais vezes do que o esperado. Ainda não existem estudos para analisar a sensibilidade da estatística (2.9) quando as variâncias são mal especificadas.

Suponha que as variâncias de $W_{k i j}$ para $k=1,2$ não são conhecidas. Uma das formas para estimar $\lambda_{i}$ e $\kappa_{i}$ é fazendo repetições em cada unidade experimental. Fazendo as réplicas então teremos

$$
Y_{i j}=c_{1 i j}^{\top} w_{1 i} \quad \text { e } \quad X_{i j}=c_{2 i j}^{\top} w_{2 i}
$$

sendo $\boldsymbol{w}_{1 i}$ e $\boldsymbol{w}_{2 i}$ vetores aleatórios independentes de dimensões $p_{1}$ e $p_{2}$ com matrizes de covariâncias $\Sigma_{1 i}$ e $\Sigma_{2 i}$ respectivamente, $c_{1 i j}$ e $c_{2 i j}$ são vetores de constantes de dimensões $p_{1}$ e $p_{2}$ respectivamente. Assim, na Seção 2.5 .1 consideramos que $\Sigma_{1 i}=$ $\lambda_{i} \boldsymbol{I}, \Sigma_{2 i}=\kappa_{i} \boldsymbol{I}$, para todo $i=1, \ldots, n ; \boldsymbol{c}_{k i j}^{\top} \boldsymbol{c}_{k i j}=1$ e $\boldsymbol{c}_{k i j}^{\top} c_{k l s}=0$ para todo $i \neq l$ ou $j \neq s$. Dessa forma, as variáveis são independentes para todo $i$ e $j$, e as variâncias são dadas por $\operatorname{Var}\left(Y_{i j}\right)=\lambda_{i}$ e $\operatorname{Var}\left(X_{i j}\right)=\kappa_{i}$.

No modelo da Seção (2.5.2) consideramos que $\Sigma_{1 i}=\lambda_{i} D_{1}$ e $\Sigma_{2 i}=\kappa_{i} \boldsymbol{D}_{2}$. Fazendo $C_{k i}^{\top}=\left(c_{k i 1}, c_{k i 2}, \ldots c_{k 1 m}\right)$ teremos que 


$$
\operatorname{Var}\left(\boldsymbol{Y}_{i}\right)=\lambda_{i} \boldsymbol{C}_{1 i} \boldsymbol{D}_{1} \boldsymbol{C}_{1 i}^{\top} \text { e } \operatorname{Var}\left(\boldsymbol{X}_{i}\right)=\kappa_{i} \boldsymbol{C}_{2 i} \boldsymbol{D}_{2} \boldsymbol{C}_{2 i}^{\top}
$$

com a seguinte restrição

$$
\frac{c_{k i j}^{\top} D_{k} c_{k i l}}{\sqrt{\boldsymbol{c}_{k i j}^{\top} \boldsymbol{D}_{k} \boldsymbol{c}_{k i j}} \sqrt{\boldsymbol{c}_{k i l}^{\top} \boldsymbol{D}_{k} \boldsymbol{c}_{k i l}}} \longrightarrow 0 \text { quando }|j-l| \rightarrow \infty
$$

para todo $k=1,2$ e para todo $i=1, \ldots, n$. Como as matrizes $\boldsymbol{D}_{1}$ e $\boldsymbol{D}_{2}$ dependem de parâmetros desconhecidos, sem perda de generalidade, podemos escrever

$$
\operatorname{Var}\left(\boldsymbol{Y}_{i}\right)=\lambda_{i} \boldsymbol{\Omega}_{i}\left(\boldsymbol{\rho}_{1}\right) \text { e } \operatorname{Var}\left(\boldsymbol{X}_{i}\right)=\kappa_{i} \boldsymbol{\Omega}_{i}\left(\boldsymbol{\rho}_{2}\right)
$$

com $\rho_{k}=\left(\rho_{k 1}, \ldots, \rho_{k p_{k}}\right)$. Geralmente assume-se que $D_{k}=\operatorname{Diag}\left(\rho_{k 1}, \ldots, \rho_{k p_{k}}\right)$, ou seja, o vetor de variáveis de entrada $\boldsymbol{w}_{k i}$ tem componentes não correlacionadas, e

$$
\operatorname{Cov}\left(Y_{i j}, Y_{i l}\right)=\lambda_{i} \sum_{t=1}^{p_{1}} \rho_{1 t} c_{1 i j t} c_{1 i l t} \quad \text { e } \operatorname{Cov}\left(X_{i j}, X_{i l}\right)=\kappa_{i} \sum_{t=1}^{p_{2}} \rho_{2 t} c_{2 i j t} c_{2 i l t}
$$

Isso indica que é razoável considerar repetições correlacionadas. Se a estrutura de covariâncias de $\boldsymbol{Y}_{i}$ e $\boldsymbol{X}_{i}$ for definida como em (2.12) tal que a restrição (2.11) seja satisfeita, então é possível estimar o vetor de parâmetros $\rho_{k}$ via MV.

\subsection{Modelo funcional para dados com réplicas}

O modelo heterocedástico apresentado na seção anterior considera que as variâncias das variáveis observadas são conhecidas ou por estudos passados ou por réplicas feitas em cada unidade experimental (u.e.). Teremos no segundo caso que as observações para a $i$-ésima u.e. são dadas por $\left(Y_{i 1}^{*}, Y_{i 2}^{*}, \ldots Y_{i m}^{*}\right)$, assim para usar os modelos da seção anterior fazemos $Y_{i}=\bar{Y}_{i}^{*}=\frac{\sum Y_{i j}^{*}}{m}$ e $\lambda_{i}=(1 / m) S_{i}^{2}=\frac{\sum\left(Y_{i j}^{*}-\bar{Y}_{i}^{*}\right)^{2}}{m^{2}}$, ou seja, a variância de $Y_{i}$ é estimada pelo método dos momentos. Dividimos $S_{i}^{2}$ por $m$ pois como $Y_{i}$ é uma média de $m$ observações espera-se que sua variância diminua 
conforme o número de observações aumente. De forma análoga obtemos a variância de $X_{i}$. Neste caso os erros na estimação de $\lambda_{i}$ e $\kappa_{i}$ não são levados em conta, pois os valores obtidos $\left(\hat{\lambda}_{i}\right.$ e $\left.\hat{\kappa}_{i}\right)$ são tratados, no processo de estimação de $\alpha$ e $\beta$, como se fossem populacionais. Uma questão que surge é: Qual o impacto nas estimativas dos parâmetros e erros-padrão, quando as variâncias são desconhecidas, num modelo funcional heterocedástico? Para responder essa questão, vamos derivar os estimadores e testes de hipóteses, considerando as repetições no processo de estimação e, por conseguinte, fazer um estudo de simulação para tentar avaliar a qualidade das metodologias.

Nesta seção definiremos um modelo que considera as réplicas geralmente feitas na prática e descartaremos a suposição de variâncias conhecidas. A estimação das variâncias é feita usando a metodologia da máxima verossimilhança. Dolby e Lipton (1972) estudaram modelos com erro nas variáveis não lineares em que existem repetições não correlacionadas e erros correlacionados. Dolby et al. (1987) consideram vários casos em que existe heterocedasticidade irrestrita $\left(\lambda_{i}, \kappa_{i}\right)$, proporcional $\left(\lambda_{i}, c \lambda_{i}\right)$ sendo $c$ uma constante conhecida e o caso homogêneo $(\lambda, \kappa)$. Neste trabalho, consideramos apenas heterocedasticidade irrestrita.

Em validação de métodos de medição, se a precisão do instrumento utilizado já for conhecida, então as repetições não serão necessárias e o modelo (2.7) pode ser usado sem maiores problemas. Se a precisão do instrumento ou método de medição não for conhecida, então as repetições são necessárias para estimar a variabilidade dos instrumentos. O problema em fazer repetições para estimar a variabilidade é que variáveis externas podem interferir no processo de medição, e a estimativa da precisão pode não ser realística. Uma forma de contornar esse problema seria considerar um modelo misto para modelar esta sobredispersão. Neste trabalho estamos assumindo que nenhuma variável externa influencia o processo de medição.

A Figura 2.3 mostra uma matriz de diagramas de dispersão para nove concentrações medidas por dois instrumentos (bola: instrumento padrão, triângulo: instrumento novo), sendo que cada instrumento replicou cem vezes a mesma concentração. 
Figura 2.3: Matriz de diagramas de dispersão de dois instrumentos de medição, para o caso em que existe repetições
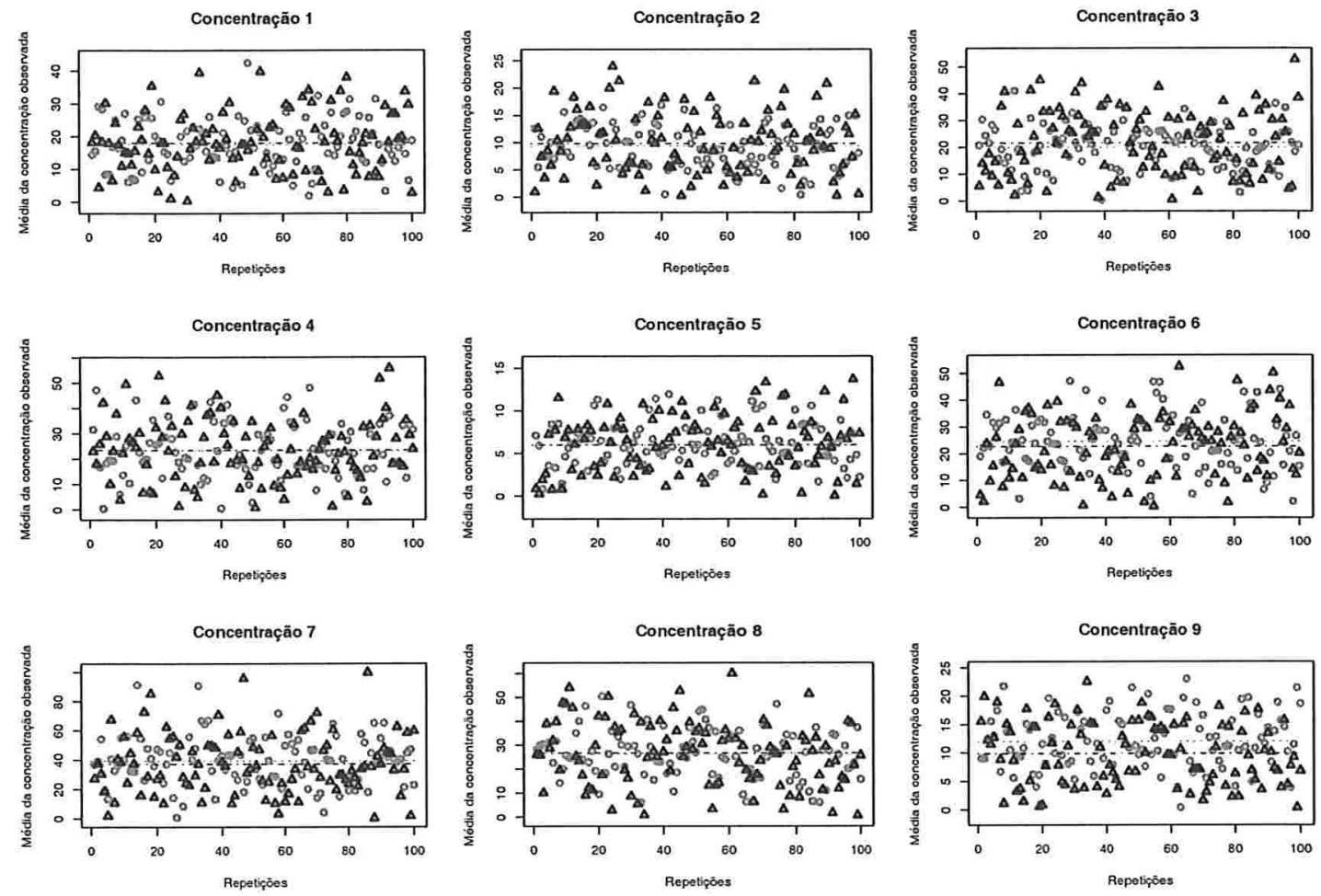

O gráfico acima foi obtido por meio de simulação sob a hipótese de que não existe viés entre os instrumentos de medição. Utilizamos a parametrização dada no Capítulo 3 na Seção (3.1), considerando concentrações simétricas - uniformes. Não é fácil perceber que $\alpha=0$ e $\beta=1$ pela Figura 2.3. Na Figura 2.4, percebemos com mais clareza que o intrumento novo não é tendencioso (em relação ao intrumento padrão). Abaixo mostramos o gráfico das médias de cada concentração. 
Figura 2.4: Gráfico das Médias

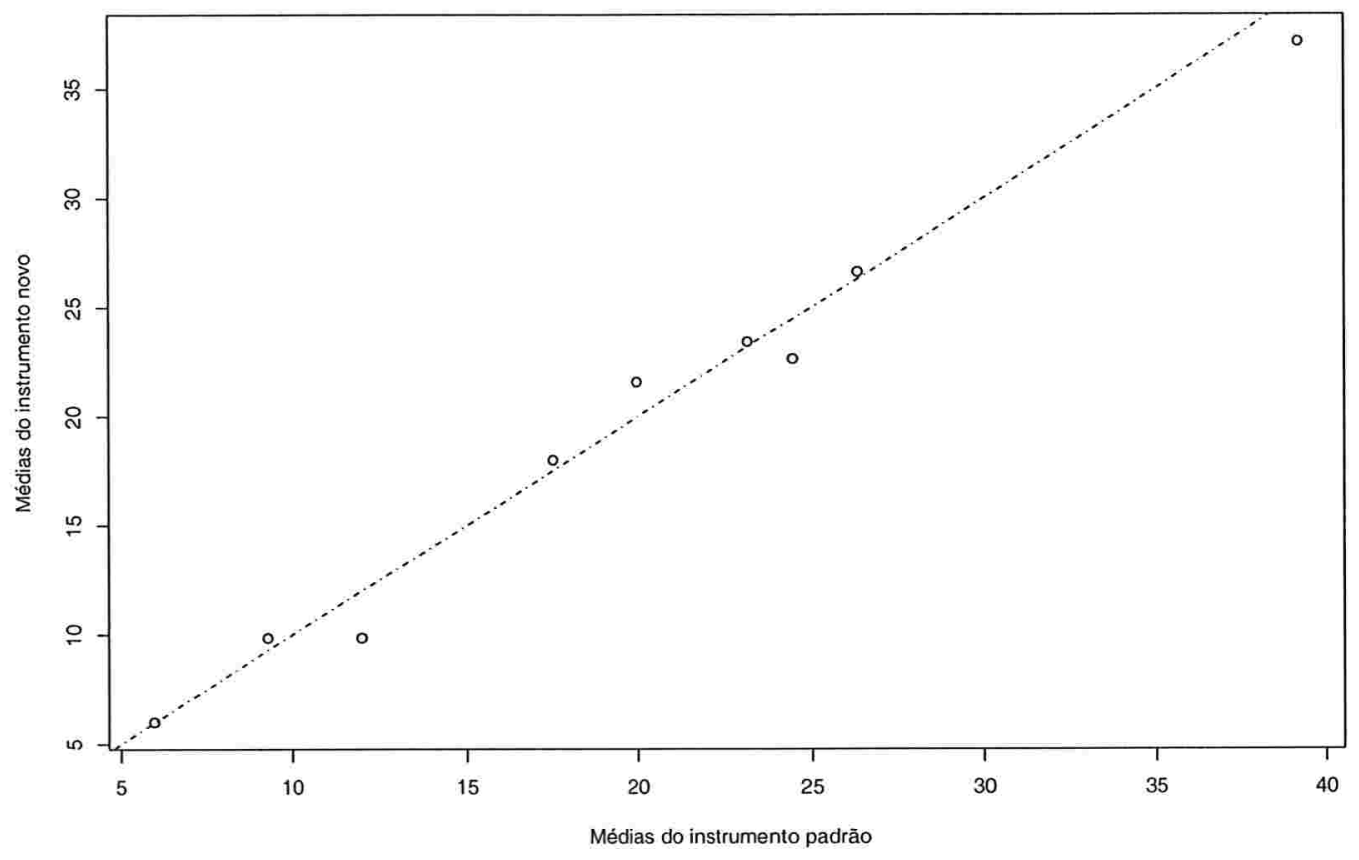

As Figuras 2.3 e 2.4 nos mostram uma situação ideal. Se não existe tendência no instrumento a ser testado, então esperamos que os gráficos tenham o mesmo comportamento dos gráficos apresentados acima. Na Figura 2.4 percebe-se que as médias dos instrumentos se distribuem aleatóriamente em torno da reta ideal.

\subsubsection{Repetições independentes}

Nesta Seção consideramos que as repetições feitas dentro de cada unidade experimental são independentes, derivamos os estimadores de MV para alguns casos específicos e contruímos uma estatística Wald para testar os parâmetros de interesse. Dolby e Lipton (1972) e Dolby et al. (1987) derivaram os estimadores de MV para os parâmetros de interesse, mas não contruíram estatísticas para testar hipóteses com 
respeito aos parâmetros.

No caso de validação de métodos o problema prático que sustenta este modelo é enunciado a seguir: Suponha que o interesse é comparar um instrumento novo $(Y)$ com o instrumento padrão $(X)$ em $n$ concentrações diferentes, e as observações de $X$ e $Y$ podem ser observadas diretamente. Suponha adicionalmente que cada concentração pode ser dividida homogeneamente em $2 m$ subamostras distintas, ou seja, se $x_{i}$ é o valor da $i$-ésima concentração teremos $(\underbrace{x_{i}, x_{i}, \ldots, x_{i}}_{2 m \text { vezes }})$ que serão divididas em dois blocos de $m$. O instrumento novo medirá o primeiro bloco e o instrumento padrão medirá o segundo. A Tabela 2.1 ilustra uma possível forma de medir as concentrações.

Tabela 2.1: Repetições feitas para a $i$-ésima concentração, para o modelo sem correlação

\begin{tabular}{l|cccc|cccc}
\hline Instrumento & \multicolumn{4}{|c}{ Novo } & \multicolumn{5}{c}{ Padrão } \\
\hline subamostra & 1 & 2 & $\ldots$ & $\mathrm{m}$ & $\mathrm{m}+1$ & $\mathrm{~m}+2$ & $\ldots$ & $2 \mathrm{~m}$ \\
\hline Verdadeira & $x_{i}$ & $x_{i}$ & $\ldots$ & $x_{i}$ & $x_{i}$ & $x_{i}$ & $\ldots$ & $x_{i}$ \\
Observado & $Y_{i 1}$ & $Y_{i 2}$ & $\ldots$ & $Y_{i m}$ & $X_{i 1}$ & $X_{i 2}$ & $\ldots$ & $X_{i m}$ \\
\hline
\end{tabular}

Da forma que o enunciado é construído, os dois métodos podem medir as concentrações de forma independente e as réplicas feitas por cada um deles também podem ser independentes. Assim, o modelo funcional neste caso pode ser escrito como

$$
\begin{aligned}
& Y_{i j}=\alpha+\beta x_{i}+e_{i j} \\
& X_{i j}=x_{i}+u_{i j}
\end{aligned}
$$

sendo $u_{i j}$ e $e_{i j}$ variáveis aleatórias independentes para todo $i \in I=\{1, \ldots, n\}$ e $j \in$ $J=\{1, \ldots, k\}$, supondo que se distribuem normalmente com média zero e variâncias 
$\kappa_{i}$ e $\lambda_{i}$, respectivamente, para todo $j \in J$. O modelo (2.13)-(2.14) pode ser reescrito da seguinte forma:

$$
Z_{i j}=\left(\begin{array}{c}
Y_{i j} \\
X_{i j}
\end{array}\right)=\left(\begin{array}{c}
\alpha \\
0
\end{array}\right)+\left(\begin{array}{c}
\beta \\
1
\end{array}\right) x_{i}+\left(\begin{array}{c}
e_{i j} \\
u_{i j}
\end{array}\right) .
$$

Vale lembrar que para utilizar o Modelo (2.15), uma condição necessária é assegurar que as variáveis externas não influenciam no processo de medição. Assim, pela suposição de normalidade dos erros, segue que a distribuição de $Z_{i j}$ é uma normal bivariada dada abaixo

$$
Z_{i j} \sim N_{2}\left[\left(\begin{array}{c}
\alpha+\beta x_{i} \\
x_{i}
\end{array}\right) ;\left(\begin{array}{cc}
\lambda_{i} & 0 \\
0 & \kappa_{i}
\end{array}\right)\right] \forall i \in I, j \in J .
$$

Como vimos no Capítulo 1 o modelo acima é conhecido na literatura como modelo funcional, porque não estamos considerando uma distribuição para a verdadeira concentração $\left(x_{i}\right)$, o modelo funcional permite uma grande flexibilidade na forma da distribuição da verdadeira covariável não observada, podendo a mesma ser assimétrica, bimodal, ter caudas muito pesadas ou muito leves, portanto é razoável supor que os erros de medidas $\left(e_{i j}\right.$ e $\left.u_{i j}\right)$ têm distribuição normal. No problema de verificação do viés nos métodos não estamos interessados diretamente nos valores da verdadeira concentração. O principal interesse, neste contexto, são os parâmetros $\alpha$ e $\beta$, pois baseado nas inferências feitas sobre eles poderemos encontrar alguma evidência de viés nos métodos. Definiremos $\boldsymbol{\theta}=(\alpha, \beta, \boldsymbol{\kappa}, \boldsymbol{\lambda}, \boldsymbol{x})$, sendo $\boldsymbol{x}=\left(x_{1}, \ldots, x_{n}\right)$, $\kappa=\left(\kappa_{1}, \ldots, \kappa_{n}\right)$ e $\lambda=\left(\lambda_{1}, \ldots, \lambda_{n}\right)$, o vetor de parâmetros desconhecidos que estimaremos via MV. Assim o logaritmo da função de verossimilhança para o modelo (2.15) é dado por

$$
\ell(\theta)=\sum_{i \in I} \sum_{j \in J} \ell_{i j}(\theta)
$$

sendo 


$$
\ell_{i j}(\boldsymbol{\theta})=-\log (2 \pi)-\frac{1}{2} \log \left(\kappa_{i}\right)-\frac{1}{2} \log \left(\lambda_{i}\right)-\frac{\left(y_{i j}-\alpha-\beta x_{i}\right)^{2}}{2 \lambda_{i}}-\frac{\left(X_{i j}-x_{i}\right)^{2}}{2 \kappa_{i}} .
$$

Para maximizar (2.16) em relação a $\theta$ precisamos encontrar as derivadas parciais em relação aos parâmetros e iguala-los a zero. No Apêndice (A.1) encontramos as derivadas de primeira ordem, que são necessárias para encontrar os estimadores abaixo

$$
\hat{x}_{i}=\frac{\kappa_{i} \beta\left(\bar{Y}_{i}-\alpha\right)+\lambda_{i} \bar{X}_{i}}{\lambda_{i}+\beta^{2} \kappa_{i}}, i \in I
$$

Nota-se que a expressão para o estimador de MV de $x_{i}$ é idêntica ao caso heterocedástico em que as variâncias $\lambda_{i}$ e $\kappa_{i}$ são conhecidas. Entretanto o valor da estimativa $\hat{x}_{i}$ será diferente nos dois casos, pois em (2.17), $\lambda_{i}$ e $\kappa_{i}$ são estimados via MV. Percebe-se também que neste caso o estimador para $x_{i}$ é uma média ponderada pelas variâncias de cada método e corrigida pelo viés do método novo.

$$
\hat{\beta}=\frac{\sum_{i \in I} \omega_{i} \hat{x}_{i}\left(\bar{Y}_{i}-\bar{Y}_{\omega}\right)}{\sum_{i \in I} \omega_{i} \hat{x}_{i}\left(\hat{x}_{i}-\hat{\bar{x}}_{\omega}\right)}, \hat{\alpha}=\bar{Y}_{\omega}-\hat{\beta} \hat{\bar{x}}_{\omega}
$$

sendo que

$$
\bar{Y}_{\omega}=\sum_{i \in I} \omega_{i} \bar{Y}_{i}, \hat{\bar{x}}_{\omega}=\sum_{i \in I} \omega_{i} \hat{x}_{i}
$$

e

$$
\omega_{i}=\frac{1}{\lambda_{i} \sum_{i \in I}\left(1 / \lambda_{i}\right)} .
$$

Os estimadores para $\alpha$ e $\beta$ também têm semelhanças com os estimadores encontrados para o modelo heterocedástico sem repetição, a diferença mais evidente é na ponderação $\omega_{i}$, que se dá pelo fato que, neste contexto, estamos usando a verossimilhança genuína e no caso em que não existem repetições, derivamos os estimadores via verossimilhança perfilada. Os estimadores para $\lambda_{i}$ e $\kappa_{i}$ são dados por 


$$
\hat{\lambda}_{i}=\frac{\sum_{j \in J}\left(Y_{i j}-\hat{\alpha}-\hat{\beta} \hat{x}_{i}\right)^{2}}{m} \text { e } \hat{\kappa}_{i}=\frac{\sum_{j \in J}\left(X_{i j}-\hat{x}_{i}\right)^{2}}{m}
$$

Notamos no Capítulo 3, na seção (3.1), que as estatísticas, formuladas mais adiante, para testar os parâmetros $\alpha$ e $\beta$ serão mais confiáveis se corrigirmos os estimadores acima por

$$
\hat{\lambda}_{i}=\frac{\sum_{j \in J}\left(Y_{i j}-\hat{\alpha}-\hat{\beta} \hat{x}_{i}\right)^{2}}{m-3} \text { e } \hat{\kappa}_{i}=\frac{\sum_{j \in J}\left(X_{i j}-\hat{x}_{i}\right)^{2}}{m-1}
$$

Para obter as estimativas de máxima verossimilhança se faz necessário um método iterativo. $\mathrm{O}$ algoritmo que utilizamos neste trabalho é do tipo-EM, ver Kimura (1992). Os passos são dados a seguir

1. Inicie o procedimento iterativo com $v=0$ e considere os valores iniciais abaixo

$$
\begin{gathered}
\alpha^{(0)}=0, \beta^{(0)}=1 \\
\lambda_{i}^{(0)}=\frac{\sum_{j \in J}\left(Y_{i j}-\bar{Y}_{i}\right)^{2}}{m} \text { e } \kappa_{i}^{(0)}=\frac{\sum_{j \in J}\left(X_{i j}-\bar{X}_{i}\right)^{2}}{m}
\end{gathered}
$$

para todo $i=\{1, \ldots, n\}$.

2. Calcule $\hat{x}_{i}^{(v)}$ em (2.17) .

3. Calcule $\hat{\beta}^{(v)}, \hat{\alpha}^{(v)}, \hat{\lambda}_{i}^{(v)}$ e $\hat{\kappa}_{i}^{(v)}$ para todo $i=\{1, \ldots, n\}$ em $(2.18)$ e $(2.19)$.

4. Incremente uma unidade a $v$.

5. Repita os passos 2, 3 e 4 até a convergência.

O critério de parada é baseado nas estimativas dos vieses e das variâncias, dado por 


$$
\epsilon^{(v)}=\max _{i \in I}\left\{\left|\frac{\hat{\alpha}^{(v)}-\hat{\alpha}^{(v-1)}}{\hat{\alpha}^{(v-1)}}\right|,\left|\frac{\hat{\beta}^{(v)}-\hat{\beta}^{(v-1)}}{\hat{\beta}^{(v-1)}}\right|,\left|\frac{\hat{\kappa}_{i}^{(v)}-\hat{\kappa}_{i}^{(v-1)}}{\hat{\kappa}_{i}^{(v-1)}}\right|,\left|\frac{\hat{\lambda}_{i}^{(v)}-\hat{\lambda}_{i}^{(v-1)}}{\hat{\lambda}_{i}^{(v-1)}}\right|\right\} .
$$

A convergência é obtida quando o valor de $\epsilon^{(v)}$ é suficientemente pequeno.

No Apêndice (A.1) mostramos que a matriz de covariâncias assintótica para os estimadores de $\alpha$ e $\beta$ é

$$
\operatorname{Cov}(\hat{\alpha}, \hat{\beta})=\left[\begin{array}{cc}
\sum_{i \in I}\left(c_{i} / \lambda_{i}\right) & \sum_{i \in I}\left(c_{i} x_{i} / \lambda_{i}\right) \\
\cdot & \sum_{i \in I}\left(c_{i} x_{i}^{2} / \lambda_{i}\right)
\end{array}\right]^{-1},
$$

sendo $c_{i}=m\left(1-\frac{\beta^{2} \kappa_{i}}{\beta^{2} \kappa_{i}+\lambda_{i}}\right)$, ver (A.14). No modelo proposto, geralmente, estamos interessados em testar a hipótese (2.6). A estatística de Wald para este teste é dada por

$$
\xi_{W_{\alpha \beta}}=\left(\begin{array}{c}
\hat{\alpha}-\alpha_{0} \\
\hat{\beta}-\beta_{0}
\end{array}\right)^{\top}\left[\begin{array}{cc}
\sum_{i \in I}\left(c_{i} / \lambda_{i}\right) & \sum_{i \in I}\left(c_{i} x_{i} / \lambda_{i}\right) \\
. & \sum_{i \in I}\left(c_{i} x_{i}^{2} / \lambda_{i}\right)
\end{array}\right]\left(\begin{array}{c}
\hat{\alpha}-\alpha_{0} \\
\hat{\beta}-\beta_{0}
\end{array}\right) .
$$

A matriz de covariâncias assintótica depende de parâmetros desconhecidos, basta usar os estimadores de MV já derivados nesta seção para estima-la consistentemente. Quando consideramos as repetições no processo de estimação, a presença de parâmetros incidentais deixa de existir, pois estamos supondo que o número de repetições $(m)$ cresce e número de concentrações $(n)$ permanece finito. Assim, poderemos reescrever o modelo da seguinte forma

$$
W_{j} \stackrel{i i d}{\sim} N_{2 n}\left(\mu^{*}, \Sigma\right) \quad \forall j=1,2, \ldots, m
$$

sendo $\boldsymbol{W}_{j}=\left(\boldsymbol{Z}_{1 j}^{\top}, Z_{2 j}^{\top} \ldots, Z_{n j}^{\top}\right)^{\top}, \boldsymbol{\mu}^{*}=\left(\boldsymbol{\mu}_{1}^{\top}, \boldsymbol{\mu}_{2}^{\top}, \ldots, \boldsymbol{\mu}_{n}^{\top}\right)^{\top}, \boldsymbol{\mu}_{i}=\left(\alpha+\beta x_{i}, x_{i}\right)^{\top}$, 


$$
\Sigma=\left(\begin{array}{cccc}
\Lambda_{1} & 0 & \ldots & 0 \\
0^{\top} & \Lambda_{2} & \ldots & 0 \\
\vdots & \vdots & \ddots & \vdots \\
0^{\top} & 0^{\top} & \ldots & \Lambda_{n}
\end{array}\right) \quad \text { e } \Lambda_{i}=\left(\begin{array}{cc}
\lambda_{i} & 0 \\
0 & \kappa_{i}
\end{array}\right)
$$

Portanto, poderemos usar o teorema do limite central multivariado e concluímos que os estimadores de MV para $\alpha$ e $\beta$ têm distribuição conjunta normal bivariada e, por conseqüência, $\xi_{W_{\alpha \beta}} \stackrel{d}{\longrightarrow} \chi^{2}(2)$. Assim, rejeita-se $H_{0}$ se o valor da estatística exceder o quantil de uma $\chi^{2}(2)$. No Capítulo 3 faremos um estudo de simulação para analisar e comparar o comportamento dos estimadores e dos testes desta seção com os da Seção (2.3).

Uma observação importante que deve ser feita é que o modelo desta seção só é válido quando as verdadeiras covariáveis podem ser divididas em blocos homogêneos e que nehuma variável externa influencia no processo de medição. Suponha que não é possível dividir as verdadeiras covariáveis em blocos homogêneos. $\mathrm{Na}$ validação de métodos as verdadeiras concentrações, do $i$-ésimo bloco, que serão medidas pelo instrumento novo são $\left(x_{y i 1}, x_{y i 2}, \ldots, x_{y i m}\right)$ e pelo instrumento padrão são $\left(x_{i 1}, x_{i 2}, \ldots, x_{i m}\right)$. Se a fonte que gera a heterogeneidade é conhecida, então assumiremos que $x_{y i j}=a_{i j} x_{i}$ e $x_{i j}=b_{i j} x_{i}$, sendo $a_{i j}$ e $b_{i j}$ constantes conhecidas para todo $i=1,2 \ldots, n$ e $j=1,2, \ldots, m$. Para ilustrar o problema consideramos que uma. indústria deseja medir a concentração de um determinando metal em $n$ terrenos, cada terreno terá uma concentração $x$. Podem existir casos em que a concentração em cada terreno não seja distribuída de forma homogênea, mas a forma como está distribuída a concentração em cada terreno é conhecida. Para comparar dois métodos de medição pode-se dividir cada terreno em dois blocos, cada bloco será medido por apenas um método e deverá ser dividido em $m$ subamostras. A figura abaixo mostra graficamente como um terreno heterogêneo com concentração $x_{1}$ pode ser dividido em dois blocos, onde cada bloco é dividio em 8 subamostras. 
Figura 2.5: Gráfico do terreno dividido em dois blocos

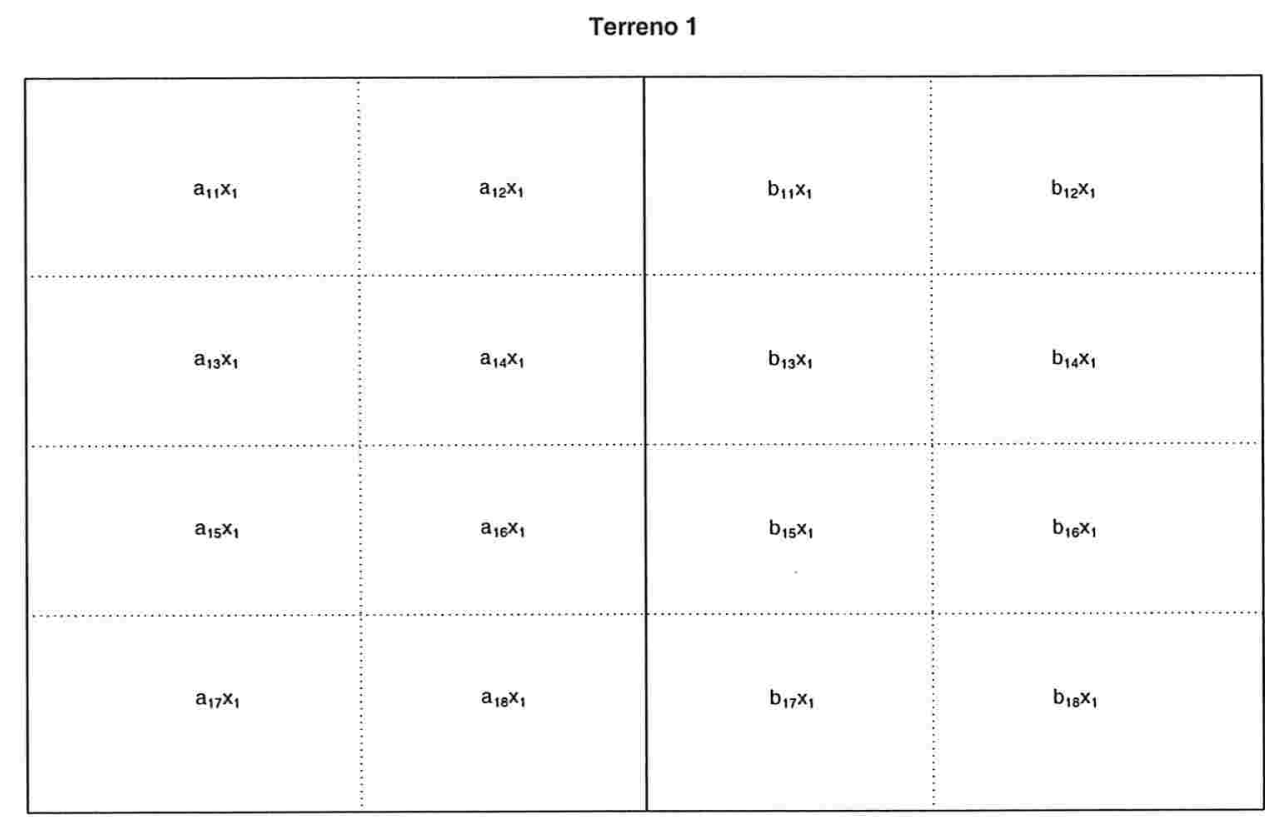

É importante notar que, se as medidas forem concentrações, deveremos ter $\sum_{j=1}^{m} a_{i j}=\sum_{j=1}^{m} b_{i j}=m$ para todo $i=1,2, \ldots, n$. Se o terreno for homogêneo então $a_{i j}=b_{i j}=1$ para todo $i$ e $j$.

Suponha que é conhecida uma relação entre $x$ e $h$, por exemplo, $x=\alpha_{0}+\beta_{0} h$, e que as quantidades $h$ podem ser observadas em cada subamostra. Seja $h_{i j}^{(1)}$ e $h_{i j}^{(2)}$ os valores de $h$ observados em cada subamostra do primeiro e segundo bloco respectivamente. Então, podemos definir $a_{i j}=\frac{1+c_{0} h_{i j}^{(1)}}{1+c_{0} \bar{h}_{i}^{(1)}}$ e $b_{i j}=\frac{1+c_{0} h_{i j}^{(2)}}{1+c_{0} \bar{h}_{i}^{(2)}}$ tal que $c_{0}=\beta_{0} / \alpha_{0}$ seja conhecido. Assim, o modelo pode ser reescrito da seguinte forma

$$
\begin{aligned}
Y_{i j} & =\alpha+\beta a_{i j} x_{i}+e_{i j} \\
X_{i j} & =b_{i j} x_{i}+u_{i j}
\end{aligned}
$$


e os estimadores obtidos nesta seção precisam ser corrigidos por

$$
\tilde{x}_{i}=\frac{\kappa_{i} \beta\left(\bar{Y}_{a i}-\bar{a}_{i} \alpha\right)+\lambda_{i} \bar{X}_{b i}}{\lambda_{i} f_{i}+\beta^{2} \kappa_{i} d_{i}}, i \in I
$$

sendo

$$
\begin{gathered}
\bar{Y}_{a i}=\sum_{j \in J} \frac{a_{i j} Y_{i j}}{m}, \bar{a}_{i}=\sum_{j \in J} \frac{a_{i j}}{m}, \bar{X}_{b i}=\sum_{j \in J} \frac{b_{i j} X_{i j}}{m}, d_{i}=\sum_{j \in J} \frac{a_{i j}^{2}}{m} \text { e } f_{i}=\sum_{j \in J} \frac{b_{i j}^{2}}{m} . \\
\tilde{\beta}=\frac{\sum_{i \in I} \omega_{i} \tilde{x}_{i}\left(\bar{Y}_{a i}-\bar{Y}_{a \omega}\right)}{\sum_{i \in I} \omega_{i} \tilde{x}_{i}\left(d_{i} \tilde{x}_{i}-\tilde{\bar{x}}_{a \omega}\right)}, \tilde{\alpha}=\bar{Y}_{a \omega}-\tilde{\beta} \tilde{\bar{x}}_{a \omega},
\end{gathered}
$$

sendo que

$$
\begin{gathered}
\bar{Y}_{a \omega}=\sum_{i \in I} \omega_{i} \bar{Y}_{a i}, \hat{\tilde{x}}_{a \omega}=\sum_{i \in I} \omega_{i} \bar{a}_{i} \tilde{x}_{i} \\
\tilde{\lambda}_{i}=\frac{\sum_{j \in J}\left(Y_{i j}-\tilde{\alpha}-\tilde{\beta} a_{i j} \tilde{x}_{i}\right)^{2}}{m} \text { e } \quad \tilde{\kappa}_{i}=\frac{\sum_{j \in J}\left(X_{i j}-b_{i j} \tilde{x}_{i}\right)^{2}}{m}
\end{gathered}
$$

A matriz de covariâncias assintótica precisa ser corrigida da seguinte forma

$$
\operatorname{Cov}(\tilde{\alpha}, \tilde{\beta})=\left[\begin{array}{cc}
\sum_{i \in I} \frac{\zeta_{i}\left(\mathbf{1}, \mathbf{1}, \boldsymbol{a}_{i}, \boldsymbol{b}_{i}\right)}{\lambda_{i}} & \sum_{i \in I} \frac{x_{i} \zeta_{i}\left(\mathbf{1}, \boldsymbol{a}_{i}, \boldsymbol{a}_{i}, \boldsymbol{b}_{i}\right)}{\lambda_{i}} \\
\sum_{i \in I} \frac{x_{i} \zeta_{i}\left(\mathbf{1}, \boldsymbol{a}_{i}, \boldsymbol{a}_{i}, \boldsymbol{b}_{i}\right)}{\lambda_{i}} & \sum_{i \in I} \frac{x_{i}^{2} \zeta_{i}\left(\boldsymbol{a}_{i}, \boldsymbol{a}_{i}, \boldsymbol{a}_{i}, \boldsymbol{b}_{i}\right)}{\lambda_{i}}
\end{array}\right]^{-1}
$$

sendo $\zeta_{i}\left(\boldsymbol{q}_{1}, \boldsymbol{q}_{2}, \boldsymbol{q}_{3}, \boldsymbol{q}_{4}\right)=\boldsymbol{q}_{1}^{\top} \boldsymbol{q}_{2}-\frac{\beta^{2} \kappa_{i}\left(\boldsymbol{q}_{3}^{\top} \boldsymbol{q}_{1}\right)\left(\boldsymbol{q}_{3}^{\top} \boldsymbol{q}_{2}\right)}{\beta^{2} \kappa_{i} \boldsymbol{q}_{3}^{\top} \boldsymbol{q}_{3}+\lambda_{i} \boldsymbol{q}_{4}^{\top} \boldsymbol{q}_{4}}$ uma função de $\boldsymbol{q}_{1}, \boldsymbol{q}_{2}, \boldsymbol{q}_{3}$ e $\boldsymbol{q}_{4}$ que são vetores de dimensão $m \times 1, \boldsymbol{a}_{i}^{\top}=\left(a_{i 1}, \ldots, a_{i m}\right)$ e $\boldsymbol{b}_{i}^{\top}=\left(b_{i 1}, \ldots, b_{i m}\right)$. A correção é baseada na formula geral (A.13). Para mais detalhes da correção veja o Apêndice (A.1).

A estatística de Wald para testar a hipótese (2.6) no caso em que não é possível dividir a verdadeira concentração homogeneamente pode ser reformulada substituindo os estimadores e a matriz de covariâncias assintótica pelo seus respectivos 
valores corrigidos. É interessante notar que se $a_{i j}=b_{i j}=1$ para todo $i, j$ voltamos ao caso mais simples em que a verdadeira concentração é dividida homogeneamente em todas as subamostras.

\subsubsection{Repetições dependentes}

Nesta seção propomos um modelo que controla a correlação entre as repetições, tais modelos não foram encontrados na literatura. A justificativa de repetições correlacionadas, na abordagem de validação de métodos, é dada na Seção 2.4 .

Abaixo apresentamos o modelo para repetições correlacionadas

$$
Z_{i}=\left(\begin{array}{c}
\boldsymbol{Y}_{i} \\
\boldsymbol{X}_{i}
\end{array}\right) \sim N_{2 m}\left[\left(\begin{array}{c}
\left(\alpha+\beta x_{i}\right) 1 \\
x_{i} \mathbf{1}
\end{array}\right) ;\left(\begin{array}{cc}
\lambda_{i} \Omega_{i}\left(\rho_{1}\right) & 0 \\
0^{\top} & \kappa_{i} \Omega_{i}\left(\rho_{2}\right)
\end{array}\right)\right] \forall i \in I,
$$

sendo que 1 é um vetor de dimensão $m$ com todos os elementos iguais a 1 , $\lambda_{i} \Omega_{i}\left(\rho_{1}\right)$ e $\kappa_{i} \Omega_{i}\left(\rho_{2}\right)$ as matrizes de covariâncias de $\boldsymbol{Y}_{i}$ e $\boldsymbol{X}_{i}$ respectivamente e $\rho_{k}=\left(\rho_{1 k}, \ldots, \rho_{p k}\right)^{\top}$ para $k=1,2$. No modelo (2.21) estamos supondo uma estrutura de correlação não muito complexa, tal que a sua derivada em relação aos parâmetros de correlação seja facilmente obtida, por exemplo, as matrizes autoregressivas e de médias móveis. Em validação de métodos, vimos que as matrizes em (2.10) podem ser usadas.

O vetor de parâmetros $\boldsymbol{\theta}=\left(\alpha, \beta, \lambda, \kappa, \boldsymbol{\rho}_{1}, \boldsymbol{\rho}_{2}, \boldsymbol{x}\right)$ é estimado via MV, ou seja, maximizando o logaritmo da função de verossimilhança para o modelo (2.21). O logaritmo natural da verossimilhança é dado por

$$
\ell(\theta)=\sum_{i \in I} \ell_{i}(\theta)
$$

com 
$\ell_{i}(\theta)=-m \log (2 \pi)-\frac{m}{2} \log \left(\kappa_{i}\right)-\frac{m}{2} \log \left(\lambda_{i}\right)-\frac{1}{2} \log \left|\Omega_{i}\left(\rho_{1}\right)\right|-\frac{1}{2} \log \left|\Omega_{i}\left(\rho_{2}\right)\right|-\frac{1}{2} A_{i}$

e

$$
A_{i}=\frac{\left(\boldsymbol{Y}_{i}-\left(\alpha+\beta x_{i}\right) \mathbf{1}\right)^{\top} \Omega_{i}\left(\rho_{1}\right)^{-1}\left(\boldsymbol{Y}_{i}-\left(\alpha+\beta x_{i}\right) \mathbf{1}\right)}{\lambda_{i}}+\frac{\left(\boldsymbol{X}_{i}-x_{i} \mathbf{1}\right)^{\top} \boldsymbol{\Omega}_{i}\left(\boldsymbol{\rho}_{2}\right)^{-1}\left(\boldsymbol{X}_{i}-x_{i} \mathbf{1}\right)}{\kappa_{i}}
$$

No apêndice (A.1) encontramos as derivadas de primeira ordem necessárias para obter os estimadores dos parâmetros em $\boldsymbol{\theta}$.

Temos,

$$
\hat{x}_{i}=\frac{\beta \kappa_{i} \mathbf{1}^{\top} \boldsymbol{\Omega}_{i}\left(\rho_{1}\right)^{-1}\left(\boldsymbol{Y}_{i}-\alpha \mathbf{1}\right)+\lambda_{i} \mathbf{1}^{\top} \Omega_{i}\left(\rho_{2}\right)^{-1} \boldsymbol{X}_{i}}{\beta^{2} \kappa_{i} \mathbf{1}^{\top} \Omega_{i}\left(\rho_{1}\right)^{-1} \mathbf{1}+\lambda_{i} \mathbf{1}^{\top} \Omega_{i}\left(\rho_{2}\right)^{-1} \mathbf{1}}, \quad i=1,2, \ldots, n .
$$

O estimador para a $i$-ésima concentração também é uma média ponderada (e corrigida pelo viés) das concentrações observadas pelos métodos de medição.

Temos, também,

$$
\hat{\beta}=\frac{\sum_{i \in I} \boldsymbol{\omega}_{i} \hat{x}_{i}\left(\boldsymbol{Y}_{i}-\bar{Y}_{\boldsymbol{\omega}} 1\right)}{\sum_{i \in I} \boldsymbol{\omega}_{i} \hat{x}_{i}\left(\hat{x}_{i}-\hat{\bar{x}}_{\boldsymbol{\omega}}\right) 1} \quad, \hat{\alpha}=\bar{Y}_{\boldsymbol{\omega}}-\hat{\beta} \hat{\bar{x}} \boldsymbol{\omega}
$$

sendo que

$$
\bar{Y}_{\omega}=\sum_{i \in I} \omega_{i} Y_{i}, \hat{\bar{x}}_{\omega}=\sum_{i \in I} \hat{x}_{i} \omega_{i} \mathbf{1}
$$

e

$$
\omega_{i}=\frac{1^{\top} \Omega_{i}\left(\rho_{1}\right)^{-1}}{\lambda_{i} \sum_{i \in I}\left(1^{\top} \Omega_{i}\left(\rho_{1}\right)^{-1} 1 / \lambda_{i}\right)} .
$$

Os estimadores para $\lambda_{i}$ e $\kappa_{i}$ são dados por

$$
\hat{\lambda}_{i}=\frac{\hat{\boldsymbol{e}}_{i}^{\top} \boldsymbol{\Omega}_{i}\left(\hat{\boldsymbol{\rho}}_{1}\right)^{-1} \hat{\boldsymbol{e}}_{i}}{m} \text { e } \hat{\kappa}_{i}=\frac{\hat{\boldsymbol{u}}_{i}^{\top} \boldsymbol{\Omega}_{i}\left(\hat{\boldsymbol{\rho}}_{2}\right)^{-1} \hat{\boldsymbol{u}}_{i}}{m}
$$


sendo $\hat{\boldsymbol{e}}_{i}=\boldsymbol{Y}_{i}-\left(\hat{\alpha}+\hat{\beta} \hat{x}_{i}\right) \mathbf{1}$ e $\hat{\boldsymbol{u}}_{i}=\boldsymbol{X}_{i}-\hat{x}_{i} \mathbf{1}$. Nota-se que os estimadores (2.23) - (2.25) são generalizações dos estimadores (2.17) - (2.19), basta tomar $\Omega_{i}\left(\rho_{1}\right)=\Omega_{i}\left(\rho_{2}\right)=\boldsymbol{I}$ (matriz identidade) e teremos a igualdade dos estimadores.

Os estimadores para $\rho_{1}$ e $\rho_{2}$ são obtidos de forma iterativa, usando o algoritmo Escore de Fisher, sendo dados por

$$
\hat{\boldsymbol{\rho}}_{k}^{(v+1)}=\hat{\boldsymbol{\rho}}_{k}^{(v)}+\left.\mathrm{E}\left[\boldsymbol{I}\left(\hat{\boldsymbol{\rho}}_{k}^{(v)}\right)\right]^{-1} \frac{\partial \ell}{\partial \rho_{k}}\right|_{\rho_{k}=\hat{\boldsymbol{\rho}}_{k}^{(v)}}
$$

os elementos de $\frac{\partial \ell}{\partial \rho_{k}}$ para $k=1,2$ são dados em (A.6) e (A.7) respectivamente; o elemento $(j, l)$ da matriz esperada de Fisher em (2.26) é dado por

$$
(1 / 2) \sum_{i=1}^{n} \operatorname{tr}\left\{\Omega_{i}\left(\rho_{k}\right)^{-1} J_{i}\left(\rho_{k}\right)_{j} \Omega_{i}\left(\rho_{k}\right)^{-1} J_{i}\left(\rho_{k}\right)_{l}\right\}, \quad \text { para } k=1,2 .
$$

A matriz $\boldsymbol{J}_{i}\left(\rho_{k}\right)_{j}$ é a matriz de derivadas de $\boldsymbol{\Omega}_{i}\left(\boldsymbol{\rho}_{k}\right)$ em relação a $\rho_{k j}$ para $k=1,2$. Um exemplo de matriz de correlação que pode ocorrer na prática é a autoregressiva de primeira ordem. Assim $\rho_{k}$ é um escalar e as matrizes definidas acima são

$$
\Omega_{i}\left(\rho_{k}\right)=\left[\begin{array}{ccccc}
1 & \rho_{k} & \rho_{k}^{2} & \ldots & \rho_{k}^{m-1} \\
\rho_{k} & 1 & \rho_{k} & \ldots & \rho_{k}^{m-2} \\
\rho_{k}^{2} & \rho_{k} & 1 & \ldots & \rho_{k}^{m-3} \\
\vdots & \vdots & \vdots & \ddots & \vdots \\
\rho_{k}^{m-1} & \rho_{k}^{m-2} & \rho^{m-3} & \ldots & 1
\end{array}\right]
$$

e

$$
\frac{\partial \Omega_{i}\left(\rho_{k}\right)}{\partial \rho_{k}}=J_{i}\left(\rho_{k}\right)=\left[\begin{array}{ccccc}
0 & 1 & 2 \rho_{k} & \ldots & (m-1) \rho_{k}^{m-2} \\
1 & 0 & 1 & \ldots & (m-2) \rho_{k}^{m-3} \\
2 \rho_{k} & 1 & 0 & \ldots & (m-3) \rho_{k}^{m-4} \\
\vdots & \vdots & \vdots & \ddots & \vdots \\
(m-1) \rho_{k}^{m-2} & (m-2) \rho_{k}^{m-3} & (m-3) \rho^{m-4} & \ldots & 0
\end{array}\right]
$$


Para encontrar as estimativas de MV dos parâmetros em questão, é suficiente saber a estrutura de correlação das repetições em cada concentração. Desta forma o processo iterativo se completa usando o tipo-EM seguindo os passos abaixo:

1. Inicie o procedimento iterativo $\operatorname{com} v=0$ e considere os valores iniciais abaixo

$$
\begin{gathered}
\alpha^{(0)}=0, \beta^{(0)}=1, \\
\lambda_{i}^{(0)}=\frac{\sum_{j \in J}\left(Y_{i j}-\bar{Y}_{i}\right)^{2}}{m}, \kappa_{i}^{(0)}=\frac{\sum_{j \in J}\left(X_{i j}-\bar{X}_{i}\right)^{2}}{m} \mathrm{e} \\
\Omega_{i}\left(\rho_{1}^{(0)}\right)=\Omega_{i}\left(\rho_{2}^{(0)}\right)=\boldsymbol{I}
\end{gathered}
$$

para todo $i=\{1, \ldots, n\}$.

2. Calcule $\hat{x}_{i}^{(v)}$ em (2.23) .

3. Calcule $\hat{\beta}^{(v)}, \hat{\alpha}^{(v)}, \hat{\lambda}_{i}^{(v)}, \hat{\kappa}_{i}^{(v)}, \hat{\rho}_{1}^{(v)}$ e ${\hat{\rho_{2}}}^{(v)}$ para todo $i=\{1, \ldots, n\}$ em $(2.24)$ (2.26).

4. Incremente uma unidade a $v$.

5. Repita os passos 2, 3 e 4 até a convergência.

O critério de parada é parecido com 2.20. Neste caso, a diferença é que o critério de parada depende também das estimativas de correlações.

No apêndice (A.1) mostramos que a matriz de covariâncias assintótica para os estimadores de $\alpha$ e $\beta$ é

$$
\operatorname{Cov}(\hat{\alpha}, \hat{\beta})=\left[\begin{array}{cc}
\sum_{i \in I}\left(c_{i}^{*} / \lambda_{i}\right) & \sum_{i \in I}\left(c_{i}^{*} x_{i} / \lambda_{i}\right) \\
\cdot & \sum_{i \in I}\left(c_{i}^{*} x_{i}^{2} / \lambda_{i}\right)
\end{array}\right]^{-1}
$$


sendo $c_{i}^{*}=\left(1^{\top} \Omega_{i}\left(\rho_{1}\right)^{-1} 1\right)\left\{1-\frac{\beta^{2} 1^{\top} \Omega_{i}\left(\rho_{1}\right)^{-1} 1 \kappa_{i}}{\beta^{2} 1^{\top} \Omega_{i}\left(\rho_{1}\right)^{-1} 1 \kappa_{i}+1^{\top} \Omega_{i}\left(\rho_{2}\right)^{-1} 1 \lambda_{i}}\right\}$, ver (A.15). Para testar a hipótese (2.6) podemos definir a estatística de Wald de forma análoga ao caso em que não temos dependência entre as repetições. Dessa forma a estatística do teste é

$$
\xi_{W_{\alpha \beta_{d}}}=\left(\begin{array}{c}
\hat{\alpha}-\alpha_{0} \\
\hat{\beta}-\beta_{0}
\end{array}\right)^{\top}\left[\begin{array}{cc}
\sum_{i \in I}\left(c_{i}^{*} / \lambda_{i}\right) & \sum_{i \in I}\left(c_{i}^{*} x_{i} / \lambda_{i}\right) \\
\cdot & \sum_{i \in I}\left(c_{i}^{*} x_{i}^{2} / \lambda_{i}\right)
\end{array}\right]\left(\begin{array}{c}
\hat{\alpha}-\alpha_{0} \\
\hat{\beta}-\beta_{0}
\end{array}\right) .
$$

Para estimar a matriz de covariâncias assintótica consistentemente basta usar os estimadores de MV já derivados. Para justificar a distribuição assintótica de $\xi_{W_{\alpha \beta_{d}}}$, reescrevemos o modelo 2.21 da seguinte forma

$$
W \sim N_{2(n+m)}(\boldsymbol{\mu}, \mathbf{\Sigma})
$$

onde, $\boldsymbol{W}=\left(Z_{1}, \ldots, Z_{n}\right), \boldsymbol{\mu}=\left(\mu_{1}, \ldots, \mu_{n}\right), \mu_{i}=\left(\alpha+\beta x_{i}, x_{i}\right)^{\top} \otimes 1$,

$$
\Sigma=\left[\begin{array}{cccc}
\Lambda_{1} & 0 & \ldots & 0 \\
0^{\top} & \Lambda_{2} & \ldots & 0 \\
\vdots & \vdots & \ddots & \vdots \\
0^{\top} & 0^{\top} & \ldots & \Lambda_{n}
\end{array}\right] \text { e } \Lambda_{i}=\left[\begin{array}{cc}
\lambda_{i} \Omega_{i}\left(\rho_{1}\right) & 0 \\
0^{\top} & \kappa_{i} \Omega_{i}\left(\rho_{2}\right)
\end{array}\right]
$$

Supondo que a estrutura escolhida para $\Omega_{i}($.$) é tal que, \operatorname{Cov}\left(e_{i j}, e_{i(j+g)}\right)$ e $\operatorname{Cov}\left(u_{i j}, u_{i(j+g)}\right)$ tendem a zero na ordem de $\exp (-|j-g|)$ quando $g$ tende ao infinto, podemos usar o resultado de Lehmann (1998, p. 106) que mostra o teorema do limite central para variáveis dependentes. Assim está justificado que $\xi_{W_{\alpha \beta_{d}}} \stackrel{d}{\longrightarrow} \chi^{2}(2)$, portanto rejeita-se $H_{0}$ se os valores das estatísticas excederem o quantil de uma $\chi^{2}(2)$.

O modelo proposto nesta seção pode ser utilizado quando o pesquisador suspeita que exista uma possível correlação entre as repetições. Neste caso, a estrutura de dependência deve ser fixada a priori. Portanto, a hipótese de interesse é $H_{0}: \Omega\left(\rho_{k}\right)=I$ (matriz identidade) contra $H_{1}: \Omega\left(\rho_{k}\right) \neq \boldsymbol{I}$ para todo $k=\{1,2\}$, ou 
seja, testar se não existe correlação entre as repetições; caso a hipótese $H_{0}$ seja verdadeira voltamos ao caso mais simples onde não existe correlação. Assim definimos o vetor $d$ tal que $\Omega(d)=I$. Dessa forma, testar se $\rho_{k}=d$ para todo o $k=1,2$ é equivalente a testar a não correlação entre as repetições, que para o modelo normal significa verificar se as repetições são independentes. A estatística de Wald para testar a independência das repetições é dada por

$$
\xi_{W \rho}=\left(\hat{\boldsymbol{\rho}}_{1}-\boldsymbol{d}\right)^{\top} \mathrm{E}\left[\boldsymbol{I}\left(\hat{\boldsymbol{\rho}}_{1}\right)\right]\left(\hat{\boldsymbol{\rho}}_{1}-\boldsymbol{d}\right)+\left(\hat{\boldsymbol{\rho}}_{2}-\boldsymbol{d}\right)^{\top} \mathrm{E}\left[\boldsymbol{I}\left(\hat{\boldsymbol{\rho}}_{2}\right)\right]\left(\hat{\boldsymbol{\rho}}_{2}-\boldsymbol{d}\right)
$$

pois $\hat{\boldsymbol{\rho}}_{1}$ e $\hat{\boldsymbol{\rho}}_{2}$ são independentes assintoticamente, ver o Apêndice (A.1). As matrizes $\mathrm{E}\left[\boldsymbol{I}\left(\hat{\boldsymbol{\rho}}_{1}\right)\right]$ e $\mathrm{E}\left[\boldsymbol{I}\left(\hat{\boldsymbol{\rho}}_{2}\right)\right]$ são as informações de Fisher esperadas para os estimadores $\hat{\rho}_{1}$ e $\hat{\rho}_{2}$ respectivamente que encontram-se em (A.1). A distribuição assintótica de $\xi_{W \rho}$ é quiquadrado com $2 p$ graus de liberdade. Se a correlação entre as repetições não for significativa, o número de parâmetros se reduz, aumentando a precisão das estimativas dos parâmetros restantes do modelo. No Capítulo 3 é feito um estudo de simulação das estatísticas $\xi_{W_{\alpha \beta_{d}}}$ para verificar seu comportamento para algumas combinações de número de concentrações e repetições.

Suponha que $\boldsymbol{Y}_{i}=\left(\alpha+\beta x_{1 i}\right) \mathbf{1}+\boldsymbol{e}_{i}$ e $\boldsymbol{X}_{i}=x_{2 i} \mathbf{1}+\boldsymbol{u}_{i}$, tal que $x_{i 1}=a_{i} x_{i}$ e $x_{i 2}=b_{i} x_{i}$, sendo $a_{i}$ e $b_{i}$ constantes conhecidas para todo $i=1,2 \ldots, n$, então os estimadores derivados nesta seção necessitam ser corrigidos por

$$
\begin{gathered}
\tilde{x}_{i}=\frac{\beta \kappa_{i} a_{i} \mathbf{1}^{\top} \boldsymbol{\Omega}_{i}\left(\rho_{1}\right)^{-1}\left(\boldsymbol{Y}_{i}-\alpha \mathbf{1}\right)+\lambda_{i} b_{i} \mathbf{1}^{\top} \boldsymbol{\Omega}_{i}\left(\rho_{2}\right)^{-1} \boldsymbol{X}_{i}}{\beta^{2} \kappa_{i} a_{i}^{2} \mathbf{1}^{\top} \boldsymbol{\Omega}_{i}\left(\rho_{1}\right)^{-1} \mathbf{1}+\lambda_{i} b_{i}^{2} \mathbf{1}^{\top} \boldsymbol{\Omega}_{i}\left(\rho_{2}\right)^{-1} \mathbf{1}}, \quad i=1,2, \ldots, n \\
\tilde{\beta}=\frac{\sum_{i \in I} \boldsymbol{\omega}_{i}\left(a_{i} \mathbf{1}\right) \tilde{x}_{i}\left(\boldsymbol{Y}_{i}-\mathbf{1} \bar{Y}_{\boldsymbol{\omega}(\mathbf{1})}\right)}{\sum_{i \in I} \boldsymbol{\omega}_{i}\left(a_{i} \mathbf{1}\right) \tilde{x}_{i}\left(a_{i} \mathbf{1} \tilde{x}_{i}-\mathbf{1} \tilde{\bar{x}} \boldsymbol{\omega}(\boldsymbol{a})\right)}, \tilde{\alpha}=\bar{Y}_{\boldsymbol{\omega}(\mathbf{1})}-\tilde{\beta} \tilde{\bar{x}} \boldsymbol{\omega}(\boldsymbol{a})
\end{gathered}
$$

sendo que

$$
\bar{Y}_{\boldsymbol{\omega}(1)}=\sum_{i \in I} \omega_{i}(1) Y_{i}, \tilde{\bar{x}}_{\boldsymbol{\omega}(\boldsymbol{a})}=\sum_{i \in I} \tilde{x}_{i} \boldsymbol{\omega}_{i}\left(a_{i} \mathbf{1}\right)
$$




$$
\omega_{i}\left(a_{i} \mathbf{1}\right)=a_{i} \frac{1^{\top} \Omega_{i}\left(\rho_{1}\right)^{-1}}{\lambda_{i} \sum_{i \in I}\left(1^{\top} \Omega_{i}\left(\rho_{1}\right)^{-1} 1 / \lambda_{i}\right)} .
$$

Os estimadores corrigidos para $\lambda_{i}$ e $\kappa_{i}$ são dados por

$$
\tilde{\lambda}_{i}=\frac{\tilde{\boldsymbol{e}}_{i}^{\top} \boldsymbol{\Omega}_{i}\left(\tilde{\boldsymbol{\rho}}_{1}\right)^{-1} \tilde{\boldsymbol{e}}_{i}}{m} \text { e } \tilde{\kappa}_{i}=\frac{\tilde{\boldsymbol{u}}_{i}^{\top} \boldsymbol{\Omega}_{i}\left(\tilde{\boldsymbol{\rho}}_{2}\right)^{-1} \tilde{\boldsymbol{u}}_{i}}{m}
$$

sendo $\tilde{\boldsymbol{e}}_{i}=\boldsymbol{Y}_{i}-\left(\tilde{\alpha}+\tilde{\beta} a_{i} \tilde{x}_{i}\right) \mathbf{1}$ e $\tilde{\boldsymbol{u}}_{i}=\boldsymbol{X}_{\boldsymbol{i}}-b_{i} \tilde{x}_{i} \mathbf{1}$. Os estimadores $\tilde{\boldsymbol{\rho}}_{1}$ e $\tilde{\boldsymbol{\rho}}_{2}$ são obtidos iterativamente usando as derivadas corrigidas em (A.1). A correção na matriz de covariâncias de $(\tilde{\alpha}, \tilde{\beta})$, ver (A.16) é dada por

$$
\operatorname{Cov}(\tilde{\alpha}, \tilde{\beta})=\left[\begin{array}{ll}
\sum_{i \in I} \frac{\zeta_{i}^{*}\left(\mathbf{1}, \mathbf{1}, a_{i} \mathbf{1}, b_{i} \mathbf{1}\right)}{\lambda_{i}} & \sum_{i \in I} \frac{x_{i} \zeta_{i}^{*}\left(\mathbf{1}, a_{i} \mathbf{1}, a_{i} \mathbf{1}, b_{i} \mathbf{1}\right)}{\lambda_{i}} \\
\sum_{i \in I} \frac{x_{i} \zeta_{i}^{*}\left(\mathbf{1}, a_{i} \mathbf{1}, a_{i} \mathbf{1}, b_{i} \mathbf{1}\right)}{\lambda_{i}} & \sum_{i \in I} \frac{x_{i}^{2} \zeta_{i}^{*}\left(a_{i} \mathbf{1}, a_{i} \mathbf{1}, a_{i} \mathbf{1}, b_{i} \mathbf{1}\right)}{\lambda_{i}}
\end{array}\right]^{-1}
$$

sendo $\zeta_{i}^{*}\left(\boldsymbol{q}_{1}, \boldsymbol{q}_{2}, \boldsymbol{q}_{3}, \boldsymbol{q}_{4}\right)=\boldsymbol{q}_{1}^{\top} \Omega_{i}\left(\rho_{1}\right)^{-1} \boldsymbol{q}_{2}-\frac{\beta^{2} \kappa_{i}\left(\boldsymbol{q}_{3}^{\top} \Omega_{i}\left(\rho_{1}\right)^{-1} \boldsymbol{q}_{1}\right)\left(\boldsymbol{q}_{3}^{\top} \Omega_{i}\left(\rho_{1}\right)^{-1} \boldsymbol{q}_{2}\right)}{\beta^{2} \kappa_{i} \boldsymbol{q}_{3}^{\top} \Omega_{i}\left(\rho_{1}\right)^{-1} \boldsymbol{q}_{3}+\lambda_{i} \boldsymbol{q}_{4}^{\top} \Omega_{i}\left(\rho_{2}\right)^{-1} \boldsymbol{q}_{4}}$, ver (A.13).

Suponha agora que $\boldsymbol{Y}_{i}=\alpha \mathbf{1}+\beta \boldsymbol{x}_{1 i}+\boldsymbol{e}_{i}$ e $\boldsymbol{X}_{i}=\boldsymbol{x}_{2 i}+\boldsymbol{u}_{i}$, tal que $\boldsymbol{x}_{i 1}=\boldsymbol{a}_{i} x_{i}$ e $\boldsymbol{x}_{i 2}=\boldsymbol{b}_{i} x_{i}$, sendo $\boldsymbol{a}_{i}=\left(a_{i 1}, \ldots, a_{i m}\right)^{\top}$ e $\boldsymbol{b}_{i}=\left(b_{i 1}, \ldots, b_{i m}\right)^{\top}$ vetores de constantes conhecidas para todo $i=1,2 \ldots, n$. Então a correção deve ser feita por

$$
\begin{gathered}
\tilde{x}_{i}=\frac{\beta \kappa_{i} \boldsymbol{a}_{i}^{\top} \Omega_{i}\left(\rho_{1}\right)^{-1}\left(Y_{i}-\alpha 1\right)+\lambda_{i} b_{i}^{\top} \Omega_{i}\left(\rho_{2}\right)^{-1} \boldsymbol{X}_{i}}{\beta^{2} \kappa_{i} \boldsymbol{a}_{i}^{\top} \Omega_{i}\left(\rho_{1}\right)^{-1} \boldsymbol{a}_{i}+\lambda_{i} \boldsymbol{b}_{i}^{\top} \Omega_{i}\left(\rho_{2}\right)^{-1} b_{i}}, \quad i=1,2, \ldots, n \\
\tilde{\beta}=\frac{\sum_{i \in I} \boldsymbol{\omega}_{i}\left(\boldsymbol{a}_{i}\right) \tilde{x}_{i}\left(Y_{i}-1 \bar{Y}_{\boldsymbol{\omega}(\mathbf{1})}\right)}{\sum_{i \in I} \boldsymbol{\omega}_{i}\left(\boldsymbol{a}_{i}\right) \tilde{x}_{i}\left(\boldsymbol{a}_{i} \tilde{x}_{i}-\mathbf{1} \tilde{\bar{x}}_{\boldsymbol{\omega}(\boldsymbol{a})}\right)}, \tilde{\alpha}=\bar{Y}_{\boldsymbol{\omega}(\mathbf{1})}-\tilde{\beta} \tilde{\bar{x}} \boldsymbol{\omega}(\boldsymbol{a})
\end{gathered}
$$

sendo que

$$
\bar{Y}_{\boldsymbol{\omega}(\mathbf{1})}=\sum_{i \in I} \boldsymbol{\omega}_{i}(\mathbf{1}) \boldsymbol{Y}_{i}, \tilde{\bar{x}}_{\boldsymbol{\omega}\left(a_{i}\right)}=\sum_{i \in I} \tilde{x}_{i} \omega_{i}\left(\boldsymbol{a}_{i}\right)
$$




$$
\omega_{i}\left(a_{i}\right)=\frac{a_{i}^{\top} \Omega_{i}\left(\rho_{1}\right)^{-1}}{\lambda_{i} \sum_{i \in I}\left(1^{\top} \Omega_{i}\left(\rho_{1}\right)^{-1} \mathbf{1} / \lambda_{i}\right)} .
$$

Os estimadores corrigidos para $\lambda_{i}$ e $\kappa_{i}$ são dados por

$$
\tilde{\lambda}_{i}=\frac{\tilde{\boldsymbol{e}}_{i}^{\top} \boldsymbol{\Omega}_{i}\left(\tilde{\boldsymbol{\rho}}_{1}\right)^{-1} \tilde{\boldsymbol{e}}_{i}}{m} \text { e } \tilde{\kappa}_{i}=\frac{\tilde{\boldsymbol{u}}_{i}^{\top} \boldsymbol{\Omega}_{i}\left(\tilde{\rho}_{2}\right)^{-1} \tilde{\boldsymbol{u}}_{i}}{m}
$$

sendo $\tilde{\boldsymbol{e}}_{i}=\boldsymbol{Y}_{i}-\left(\tilde{\alpha} 1+\tilde{\beta} \boldsymbol{a}_{i} \tilde{x}_{i}\right)$ e $\tilde{\boldsymbol{u}}_{i}=\boldsymbol{X}_{i}-\boldsymbol{b}_{i} \tilde{x}_{i}, \operatorname{com} \boldsymbol{a}_{i}=\left(a_{i 1}, a_{i 2}, \ldots, a_{i m}\right)^{\top} \mathrm{e}$ $\boldsymbol{b}_{i}=\left(b_{i 1}, b_{i 2}, \ldots, b_{i m}\right)^{\top}$. Os estimadores $\tilde{\boldsymbol{\rho}}_{1}$ e $\tilde{\boldsymbol{\rho}}_{2}$ são obtidos iterativamente usando as derivadas corrigidas em (A.1). A correção na matriz de covariâncias assintótica de $(\tilde{\alpha}, \tilde{\beta})$ é dada por

$$
\operatorname{Cov}(\tilde{\alpha}, \tilde{\beta})=\left[\begin{array}{ll}
\sum_{i \in I} \frac{\zeta_{i}^{*}\left(\mathbf{1}, \mathbf{1}, \boldsymbol{a}_{i}, \boldsymbol{b}_{i}\right)}{\lambda_{i}} & \sum_{i \in I} \frac{x_{i} \zeta_{i}^{*}\left(\mathbf{1}, \boldsymbol{a}_{i}, \boldsymbol{a}_{i}, \boldsymbol{b}_{i}\right)}{\lambda_{i}} \\
\sum_{i \in I} \frac{x_{i} \zeta_{i}^{*}\left(\mathbf{1}, \boldsymbol{a}_{i}, \boldsymbol{a}_{i}, \boldsymbol{b}_{i}\right)}{\lambda_{i}} & \sum_{i \in I} \frac{x_{i}^{2} \zeta_{i}^{*}\left(\boldsymbol{a}_{i}, \boldsymbol{a}_{i}, \boldsymbol{a}_{i}, \boldsymbol{b}_{i}\right)}{\lambda_{i}}
\end{array}\right]^{-1}
$$

Fazendo $a_{i}=a_{i} \mathbf{1}$ e $b_{i}=b_{i} \mathbf{1}$ para todo o $i \in I$, voltamos ao caso em que $\boldsymbol{Y}_{i}=\left(\alpha+\beta x_{1 i}\right) \mathbf{1}+\boldsymbol{e}_{i}$ e $\boldsymbol{X}_{i}=x_{2 i} \mathbf{1}+\boldsymbol{u}_{i}$, e fazendo $\boldsymbol{a}_{i}=1$ e $\boldsymbol{b}_{i}=\mathbf{1}$ para todo o $i \in I$ voltamos ao caso mais simples $\boldsymbol{Y}_{i}=\left(\alpha+\beta x_{i}\right) \mathbf{1}+\boldsymbol{e}_{i}$ e $\boldsymbol{X}_{i}=x_{i} \mathbf{1}+\boldsymbol{u}_{i}$.

\subsubsection{Extensão para comparação de várias retas}

Os modelos apresentados nas seções acima consideram uma reta de regressão que depende de uma covariável não observada. Fazendo um paralelo com validação de métodos, uma reta de regressão significa comparar apenas dois métodos. Uma. extensão natural seria incorporar no modelo que vários métodos estão mensurando a mesma quantidade. Às vezes é interessante testar se todos os métodos (instrumentos) de medição mensuram a concentração desejada sem tendência alguma. Esta seção consta apenas como extensão dos modelos e não faremos um estudo de simulação e nem apresentaremos uma aplicação para tal caso. 
Galea-Rojas et al. (2003), Castro et al. (2004) e Galea-Rojas et al. (2005) também estudam metodologias para comparar vários métodos de medição quando não existem replicações. Estes autores consideram o modelo funcional heterocedástico com variâncias conhecidas. Taylor (1973) estudou o modelo funcional homocedástico para comparar vários métodos de medição. No artigo, Taylor considera repetições em cada unidade experimental e propõe uma estatística da razão de verossimilhanças para testar os parâmetros de interesse. Nesta seção derivamos os estimadores de MV e propomos uma estatística de Wald para testar hipóteses sobre os parâmetros.

Suponha que o interesse agora é comparar $r$ instrumentos novos $\left(Y_{1}, \ldots, Y_{r}\right)$ com o instrumento padrão $(X)$ em $n$ concentrações diferentes. Suponha adicionalmente que cada concentração pode ser dividida homogeneamente e nenhuma variável externa interfere na medições.

O modelo que propomos para comparar os $r+1$ métodos de medição considera a dependência das repetições. Se a independência for satisfeita o mesmo modelo pode ser usado com algumas restrições. Abaixo descrevemos o modelo utilizado para comparar os vários instrumentos de interesse

$$
\begin{aligned}
Y_{i l} & =\left(\alpha_{l}+\beta_{l} x_{i}\right) \mathbf{1}+\boldsymbol{e}_{i l} \\
\boldsymbol{X}_{i} & =x_{i} \mathbf{1}+\boldsymbol{u}_{i}
\end{aligned}
$$

sendo $\boldsymbol{e}_{i l}$ e $\boldsymbol{u}_{i}$ vetores aleatórios independentes para todo $i \in I=\{1, \ldots, n\}$ e $l \in L=$ $\{1, \ldots, r\}$ com distribuição normal $m$ - variada, vetor de médias igual a zero e matriz de covariâncias $\lambda_{i l} \Omega_{i l}\left(\rho_{1 l}\right)$ e $\kappa_{i} \Omega_{i}\left(\rho_{2}\right)$, respectivamente, com $\rho_{1 l}=\left(\rho_{11 l}, \ldots, \rho_{1 p l}\right)$ para $l=1,2, \ldots, r$ e $\rho_{2}=\left(\rho_{21}, \ldots, \rho_{2 p}\right)$.

Em (2.28) e (2.29) o interesse principal é verificar se todos os instrumentos estão calibrados conjuntamente com o instrumento de referência $X$, ou seja, testar se $\left(\alpha_{l}, \beta_{l}\right)=(0,1)$ para todo o $l=1,2, \ldots, r$. Assim, se o teste for aceito, concluímos que todos os instrumentos (métodos) são iguais ao instrumento padrão a menos de um erro aleatório que tenha média zero. 
O modelo proposto pode ser escrito da seguinte forma

$$
\left(\begin{array}{c}
\boldsymbol{Y}_{i l} \\
\boldsymbol{X}_{i}
\end{array}\right)=\left(\begin{array}{c}
\left(\alpha_{l}+\beta_{l} x_{i}\right) 1 \\
x_{i} \mathbf{1}
\end{array}\right)+\left(\begin{array}{c}
\boldsymbol{e}_{i l} \\
\boldsymbol{u}_{i}
\end{array}\right) .
$$

Pela suposição de normalidade dos erros, segue que a distribuição de $\left(\boldsymbol{Y}_{i l}, \boldsymbol{X}_{i}\right)$ é uma normal $2 m$-variada dada abaixo

$$
\left(\begin{array}{c}
\boldsymbol{Y}_{i l} \\
\boldsymbol{X}_{i}
\end{array}\right) \sim N_{2 m}\left[\left(\begin{array}{c}
\left(\alpha_{l}+\beta_{l} x_{i}\right) \mathbf{1} \\
x_{i} \mathbf{1}
\end{array}\right) ;\left(\begin{array}{cc}
\lambda_{i l} \Omega_{i l}\left(\rho_{1 l}\right) & 0 \\
\mathbf{0}^{\top} & \kappa_{i} \Omega_{i}\left(\rho_{2}\right)
\end{array}\right)\right] \forall i \in I .
$$

O vetor de parâmetros para o modelo (2.30) é $\boldsymbol{\theta}=\left(\boldsymbol{\alpha}, \boldsymbol{\beta}, \boldsymbol{\lambda}, \boldsymbol{\kappa}, \boldsymbol{\rho}_{1}, \boldsymbol{\rho}_{2}, \boldsymbol{x}\right)$, sendo $\alpha=\left(\alpha_{1}, \ldots, \alpha_{r}\right), \boldsymbol{\beta}=\left(\beta_{1}, \ldots, \beta_{r}\right), \kappa=\left(\kappa_{11}, \ldots, \kappa_{n r}\right), \boldsymbol{\lambda}=\left(\lambda_{11}, \ldots, \lambda_{n r}\right)$ e $\rho_{1}=$ $\left(\rho_{11}, \ldots, \rho_{1 r}\right)$. O logaritmo da função de verossimilhança para o modelo (2.30) é dado por

$$
\ell(\theta)=\sum_{i \in I} \ell_{i}(\theta)
$$

sendo

$$
\begin{aligned}
\ell_{i}(\boldsymbol{\theta})= & -\frac{(r+2 m)}{2} \log (2 \pi)-\frac{m}{2} \log \left(\kappa_{i}\right)-\frac{1}{2} \log \left|\Omega_{i}\left(\rho_{2}\right)\right|-\frac{m}{2} \sum_{l=1}^{r} \log \left(\lambda_{i l}\right)- \\
& -\frac{1}{2} \sum_{l=1}^{r} \log \left|\Omega_{i l}\left(\rho_{1 l}\right)\right|-\frac{1}{2} A_{i}
\end{aligned}
$$

$\mathrm{e}$

$$
\begin{aligned}
A_{i}=\sum_{l=1}^{r}\left\{\frac{\left(\boldsymbol{Y}_{i l}-\left(\alpha_{l}+\beta_{l} x_{i}\right) 1\right)^{\top} \Omega_{i l}\left(\rho_{1 l}\right)^{-1}\left(\boldsymbol{Y}_{i l}-\left(\alpha_{l}+\beta_{l} x_{i}\right) 1\right)}{\lambda_{i l}}\right\} \\
+\frac{\left(\boldsymbol{X}_{i}-x_{i} 1\right)^{\top} \Omega_{i}\left(\rho_{2}\right)^{-1}\left(\boldsymbol{X}_{i}-x_{i} 1\right)}{\kappa_{i}} .
\end{aligned}
$$

As duas primeras derivadas do logaritmo da verossimilhança com respeito a $\boldsymbol{\theta}$ são dadas no Apêndice (A.2). Abaixo mostramos os estimadores de MV para os parâmetros 


$$
\hat{x}_{i}=\frac{\kappa_{i} \sum_{l \in L}\left[\frac{\beta_{l} \mathbf{1}^{\top} \Omega_{i l}\left(\rho_{1 l}\right)^{-1}\left(\boldsymbol{Y}_{i l}-\alpha_{l} \mathbf{1}\right)}{\lambda_{i l}}\right]+\mathbf{1}^{\top} \Omega_{i}\left(\rho_{2}\right)^{-1} \boldsymbol{X}_{i}}{\kappa_{i} \sum_{l \in L}\left[\frac{\beta_{l}^{2} \mathbf{1}^{\top} \Omega_{i l}\left(\rho_{1 l}\right)^{-1} \mathbf{1}}{\lambda_{i l}}\right]+\mathbf{1}^{\top} \Omega_{i}\left(\rho_{2}\right)^{-1} \mathbf{1}} .
$$

O estimador para a $i$-ésima concentração envolve as observações de todos os métodos de medição, é uma média ponderada pelas variâncias de cada método e corrigida pelos respectivos vieses. Temos, também,

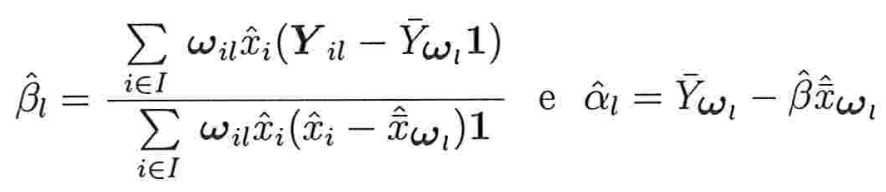

sendo que

$$
\bar{Y}_{\omega_{l}}=\sum_{i \in I} \omega_{i l} Y_{i l}, \hat{\bar{x}}_{\omega_{i l}}=\sum_{i \in I} \hat{x}_{i} \omega_{i l} 1
$$

e

$$
\omega_{i l}=\frac{1^{\top} \Omega_{i l}\left(\rho_{1 l}\right)^{-1}}{\lambda_{i l} \sum_{i \in I}\left(1^{\top} \Omega_{i l}\left(\rho_{1 l}\right)^{-1} 1 / \lambda_{i l}\right)} .
$$

Os estimadores para $\lambda_{i l}$ e $\kappa_{i}$ são dados por

$$
\hat{\lambda}_{i l}=\frac{\hat{e}_{i l}^{\top} \Omega_{i l}\left(\rho_{1 l}\right)^{-1} \hat{e}_{i l}}{m} \text { e } \hat{\kappa}_{i}=\frac{\hat{u}_{i}^{\top} \Omega_{i}\left(\rho_{2}\right)^{-1} \hat{u}_{i}}{m}
$$

sendo $\hat{\boldsymbol{e}}_{i l}=\boldsymbol{Y}_{i l}-\left(\hat{\alpha}_{l}+\hat{\beta}_{l} \hat{x}_{i}\right) \mathbf{1}$ e $\hat{\boldsymbol{u}}_{i}=\boldsymbol{X}_{i}-\hat{x}_{i} 1$. Os estimadores para $\boldsymbol{\rho}_{1 l}$ e $\boldsymbol{\rho}_{2}$ são obtidos de forma iterativa, usando o algoritmo Escore de Fisher dado por

$$
\begin{aligned}
& \hat{\rho}_{1 l}^{(v+1)}=\hat{\rho}_{1 l}^{(v)}+\left.\mathrm{E}\left[I\left(\hat{\boldsymbol{\rho}}_{1 l}^{(v)}\right)\right]^{-1} \frac{\partial \ell}{\partial \rho_{1 l}}\right|_{\rho_{1 l}=\hat{\rho}_{1 l}^{(v)}} \\
& \hat{\rho}_{2}^{(v+1)}=\hat{\rho}_{2}^{(v)}+\left.\mathrm{E}\left[I\left(\hat{\rho}_{2}^{(v)}\right)\right]^{-1} \frac{\partial \ell}{\partial \rho_{2}}\right|_{\rho_{2}=\hat{\rho}_{2}^{(v)}},
\end{aligned}
$$

com os elementos de $\frac{\partial \ell}{\partial \rho_{1 l}}$ e $\frac{\partial \ell}{\partial \rho_{2}}$ dados em (A.22) e (A.23) respectivamente. O elemento $(s, b)$ da matriz $\mathrm{E}\left[\boldsymbol{I}\left(\hat{\boldsymbol{\rho}}_{1 l}^{(v)}\right)\right]$ é dado por 


$$
(1 / 2) \sum_{i=1}^{n} \operatorname{tr}\left\{\Omega_{i l}\left(\rho_{1 l}\right)^{-1} J_{i l}\left(\rho_{1 l}\right)_{s} \Omega_{i l}\left(\rho_{1 l}\right)^{-1} J_{i l}\left(\rho_{1 l}\right)_{b}\right\}
$$

e o elemento $(s, b)$ da matriz $\mathrm{E}\left[\boldsymbol{I}\left(\hat{\boldsymbol{\rho}}_{2}^{(v)}\right)\right]$ é dado por

$$
(1 / 2) \sum_{i=1}^{n} \operatorname{tr}\left\{\Omega_{i}\left(\rho_{2}\right)^{-1} \boldsymbol{J}_{i}\left(\rho_{2}\right)_{s} \Omega_{i}\left(\rho_{2}\right)^{-1} \boldsymbol{J}_{i}\left(\rho_{2}\right)_{b}\right\},
$$

onde $\boldsymbol{J}_{i l}\left(\rho_{1 l}\right)_{s}$ e $\boldsymbol{J}_{i}\left(\rho_{2}\right)_{s}$ são as matrizes de derivadas de $\Omega_{i l}\left(\rho_{1 l}\right)$ em relação a $\rho_{1 l s}$ e $\Omega_{i l}\left(\rho_{1 l}\right)$ em relação a $\rho_{2 s}$ respectivamente. O processo iterativo para calcular as estimativas dos parâmetros se completa usando o tipo-EM seguindo os passos abaixo.

1. Inicie o procedimento iterativo com $v=0$ e considere os valores iniciais

$$
\begin{gathered}
\boldsymbol{\alpha}^{(0)}=0, \boldsymbol{\beta}^{(0)}=1 \\
\lambda_{i}^{(0)}=\frac{\sum_{j \in J}\left(Y_{i j}-\bar{Y}_{i}\right)^{2}}{m}, \kappa_{i}^{(0)}=\frac{\sum_{j \in J}\left(X_{i j}-\bar{X}_{i}\right)^{2}}{m} \mathrm{e} \\
\Omega_{i l}\left(\rho_{1 l}^{(0)}\right)=\Omega_{i}\left(\rho_{2}^{(0)}\right)=I, \forall l \in\{1,2, \ldots, p\}
\end{gathered}
$$

para todo $i=\{1, \ldots, n\}$.

2. Calcule $\hat{x}_{i}^{(v)}$ em (2.23) .

3. Calcule $\hat{\boldsymbol{\beta}}^{(v)}, \hat{\boldsymbol{\alpha}}^{(v)}, \hat{\lambda}_{i}^{(v)}, \hat{\kappa}_{i}^{(v)}, \hat{\boldsymbol{\rho}}_{1 l}^{(v)}$ e ${\hat{\rho_{2}}}^{(v)}$ para todo $i=\{1, \ldots, n\}$ em (2.33)(2.36).

4. Incremente uma unidade a $v$.

5. Repita os passos 2, 3 e 4 até a convergência.

O critério de parada é baseado nas estimativas dos vieses aditivos e multiplicativos, das variâncias e das correlações. 
Os estimadores para o caso em que as repetições são independentes em todos os métodos são obtidos fazendo: $\Omega_{i l}\left(\rho_{1 l}\right)=\Omega_{i}\left(\rho_{2}\right)=I$ (matriz identidade) para todo $i=1, \ldots, n$ e $l=1,2, \ldots, p$. Dessa forma os estimadores para o caso de independência das repetições são dados por

$$
\begin{gathered}
\hat{x}_{i}=\frac{\kappa_{i} \sum_{l \in L}\left[\frac{\beta_{l}\left(\bar{Y}_{i l}-\alpha_{l}\right)}{\lambda_{i l}}\right]+\bar{X}_{i}}{\kappa_{i} \sum_{l \in L}\left[\frac{\beta_{l}^{2}}{\lambda_{i l}}\right]+1}, \\
\hat{\beta}_{l}=\frac{\sum_{i \in I} \omega_{i l} \hat{x}_{i}\left(\bar{Y}_{i l}-\bar{Y}_{\omega_{l}}\right)}{\sum_{i \in I} \omega_{i l} \hat{x}_{i}\left(\hat{x}_{i}-\hat{\bar{x}}_{\omega_{l}}\right)} \text { e } \hat{\alpha}_{l}=\bar{Y}_{\omega_{l}}-\hat{\beta} \hat{\bar{x}}_{\omega_{l}}
\end{gathered}
$$

sendo que

$$
\bar{Y}_{\omega_{l}}=\sum_{i \in I} \omega_{i l} \bar{Y}_{i l}, \hat{\bar{x}}_{\omega_{i l}}=\sum_{i \in I} \hat{x}_{i} \omega_{i l}
$$

$\mathrm{e}$

$$
\omega_{i l}=\frac{1}{\lambda_{i l} \sum_{i \in I}\left(1 / \lambda_{i l}\right)} .
$$

Os estimadores para $\lambda_{i l}$ e $\kappa_{i}$ são dados por

$$
\hat{\lambda}_{i l}=\frac{\hat{\boldsymbol{e}}_{i l}^{\top} \hat{\boldsymbol{e}}_{i l}}{m} \text { e } \hat{\kappa}_{i}=\frac{\hat{\boldsymbol{u}}_{i}^{\top} \hat{\boldsymbol{u}}_{i}}{m},
$$

sendo $\hat{\boldsymbol{e}}$ e $\hat{\boldsymbol{u}}$ dados em (2.34). O algoritmo do tipo EM para encontrar as estimativas é o mesmo, apenas não é necessário encontrar as estimativas para $\rho_{1 l}$ e $\rho_{2}$, e o critério de parada só depende dos vieses e das variâncias.

Para usar o modelo que supõe independência entre as réplicas, devemos testar a hipótese $H_{0}: \Omega_{i l}\left(\rho_{1 l}\right)=\Omega_{i}\left(\rho_{2}\right)=I$ (matriz identidade) contra $H_{1}$ : pelo menos um diferente, para todo $i \in I$ e $l \in L$. Assim definiremos o vetor $d$ tal que $\Omega(d)=I$, então testar se $\rho_{1 l}=\rho_{2}=d$ para todo o $l=1,2, \ldots, r$ é equivalente a testar a não correlação entre as repetições de todos os métodos. A estatística de Wald para 
verificar a independência das repetições é dada por

$$
\xi_{W} \rho=\sum_{l=1}^{r}\left(\hat{\rho}_{1 l}-d\right)^{\top} \mathrm{E}\left[\boldsymbol{I}\left(\hat{\boldsymbol{\rho}}_{1 l}\right)\right]\left(\hat{\boldsymbol{\rho}}_{1 l}-d\right)+\left(\hat{\boldsymbol{\rho}}_{2}-\boldsymbol{d}\right)^{\top} \mathrm{E}\left[\boldsymbol{I}\left(\hat{\boldsymbol{\rho}}_{2}\right)\right]\left(\hat{\boldsymbol{\rho}}_{2}-\boldsymbol{d}\right) .
$$

As matrizes de informação de Fisher esperadas E $\left[\boldsymbol{I}\left(\hat{\boldsymbol{\rho}}_{1 l}\right)\right]$ e $\mathrm{E}\left[\boldsymbol{I}\left(\hat{\boldsymbol{\rho}}_{2}\right)\right]$ são dadas no Apêndice (A.2) em (A.24) - (A.27). Pelas propriedades dos estimadores de MV, a distribuição assintótica de $\xi_{W} \rho$ é qui-quadrado com $p(r+1)$ graus de liberdade.

O interesse principal é verificar se existe viés entre os instrumentos de medição. Portanto desejamos testar $H_{0}:\left(\alpha_{l}, \beta_{l}\right)=(0,1), \forall l=1,2, \ldots, r$ contra $H_{1}:\left(\alpha_{l}, \beta_{l}\right) \neq$ $(0,1)$, para pelo menos um $l$. A estatística de Wald para o teste é dada por

$$
\xi_{W} \boldsymbol{\alpha}=\left(\begin{array}{c}
\hat{\boldsymbol{\alpha}} \\
\hat{\boldsymbol{\beta}}-\mathbf{1}
\end{array}\right)^{\top}\left[\begin{array}{cc}
\sum_{i \in I}\left\{\boldsymbol{F}_{i}-\frac{\boldsymbol{D}_{i} \boldsymbol{D}_{i}^{\top}}{a_{i}}\right\} & \sum_{i \in I} x_{i}\left\{\boldsymbol{F}_{i}-\frac{\boldsymbol{D}_{i} \boldsymbol{D}_{i}^{\top}}{a_{i}}\right\} \\
. & \sum_{i \in I} x_{i}^{2}\left\{\boldsymbol{F}_{i}-\frac{\boldsymbol{D}_{i} \boldsymbol{D}_{i}^{\top}}{a_{i}}\right\}
\end{array}\right]\left(\begin{array}{c}
\hat{\boldsymbol{\alpha}} \\
\hat{\boldsymbol{\beta}}-\mathbf{1}
\end{array}\right)
$$

sendo

$$
\begin{gathered}
\boldsymbol{D}_{i}^{\top}=\left[\frac{\beta_{1}}{\lambda_{i 1}}\left(\mathbf{1}^{\top} \Omega_{i 1}\left(\rho_{11}\right)^{-1} \mathbf{1}\right), \ldots, \frac{\beta_{r}}{\lambda_{i r}}\left(\mathbf{1}^{\top} \Omega_{i r}\left(\rho_{1 r}\right)^{-1} \mathbf{1}\right)\right] \\
\boldsymbol{F}_{i}=\operatorname{diag}\left\{\frac{\beta_{1}}{\lambda_{i 1}}\left(\mathbf{1}^{\top} \Omega_{i 1}\left(\rho_{11}\right)^{-1} 1\right), \ldots, \frac{\beta_{r}}{\lambda_{i r}}\left(1^{\top} \Omega_{i r}\left(\rho_{1 r}\right)^{-1} \mathbf{1}\right)\right\}
\end{gathered}
$$

e

$$
a_{i}=\sum_{q=1}^{r} \frac{\beta_{q}^{2} \kappa_{i} \mathbf{1}^{\top} \Omega_{i q}\left(\rho_{1 q}\right)^{-1} \mathbf{1}+\lambda_{i q} \mathbf{1}^{\top} \Omega_{i}\left(\rho_{2}\right)^{-1} \mathbf{1}}{\lambda_{i q} \kappa_{i}}
$$

e para o caso em que as réplicas são independentes teremos

$$
\begin{gathered}
D_{i}^{\top}=m\left[\frac{\beta_{1}}{\lambda_{i 1}}, \ldots, \frac{\beta_{r}}{\lambda_{i r}}\right], \\
\boldsymbol{F}_{i}=m \operatorname{diag}\left\{\frac{\beta_{1}}{\lambda_{i 1}}, \ldots, \frac{\beta_{r}}{\lambda_{i r}}\right\}
\end{gathered}
$$

e

$$
a_{i}=m \sum_{q=1}^{r} \frac{\beta_{q}^{2} \kappa_{i}+\lambda_{i q}}{\lambda_{i q} \kappa_{i}}
$$


Para mais detalhes em como obter as matrizes de covariâncias assintóticas veja o Apêndice (A.2). Podemos reescrever o modelo (2.30) da mesma forma que em (2.27). E para que os estimadores de máxima verossimilhança tenham as propriedades assintóticas válidas, é necessário que as estruturas escolhidas para $\Omega_{i l}($.) e $\Omega_{i}($.$) sejam tais que, \operatorname{Cov}\left(e_{i l j}, e_{i l(j+g)}\right)$ e $\operatorname{Cov}\left(u_{i j}, u_{i(j+g)}\right)$ tendem a zero na ordem de $\exp (-|j-g|)$ quando $g$ tende ao infinto, ver Lehmann (1998). Assim, a distribuição assintótica de $\xi_{W} \boldsymbol{\alpha} \boldsymbol{\beta}$ é quiquadrado com $2 r$ graus de liberdade.

\subsection{Resumo do capítulo}

Com os procedimentos apresentados neste capítulo é possível tratar diversos tipos de situações na validação de métodos:

1) Comparar dois instrumentos de medição quando não se tem repetições.

- No caso homocedástico é necessário supor que um dos métodos tem variância conhecida, ou que a razão das variâncias é conhecida;

- No caso heterocedástico é necessário supor variâncias, de ambos os métodos, conhecidas;

2) Comparar dois instrumentos de medição quando repetições são feitas em cada unidade experimental.

- No caso em que as repetições são independentes, apresentamos um procedimento para calcular as estimativas de MV para os parâmetros do modelo e testes para detectar a tendência do instrumento;

- No caso em que as repetições não são independentes, apresentamos um procedimento para calcular as estimativas de MV para os parâmetros do modelo, 
testes para detectar a tendência do instrumento e testes para verificar se realmente existe alguma correlação entre as observações replicadas (supondo que a estrutura de correlação está correta);

- Estendemos os resultados acima para o caso em que variáveis externas influenciam no procedimento de medição.

3) Comparar vários instrumentos de medição quando repetições são feitas em cada unidade experimental.

- No caso em que as repetições são independentes, apresentamos um procedimento para calcular as estimativas de MV para os parâmetros do modelo e testes para detectar a tendência do instrumento;

- No caso em que as repetições não são independentes, apresentamos um procedimento para calcular as estimativas de MV para os parâmetros do modelo, testes para detectar a tendência do instrumento e testes para verificar se realmente existe alguma correlação entre as observações replicadas (supondo que a estrutura de correlação está correta); 


\section{Capítulo 3}

\section{Simulação}

Neste capítulo faremos uso de simulações para comparar os modelos apresentados nesta dissertação. Técnicas de Monte Carlo são utilizadas para calcular o erro quadrático médio (EQM), viés dos estimadores e nível de significância dos testes de forma empírica. A idéia é estudar o comportamento dos estimadores (viés e EQM) e testes de hipóteses (Erros tipo I e II) apresentados no capítulo anterior quando o número de repetições é pequeno. É importante saber qual o número de repetições necessário para obter resultados confiáveis.

Nas Seções (3.1) e (3.2) constam, respectivamente, as simulações para o caso em que as repetições são independentes e dependentes com estrutura de correlação autoregressiva de ordem 1. Em cada seção consideramos concentrações simétricas e assimétricas. A probabilidade do Erro tipo I (nível de significância) será estimada gerando um modelo sob a hipótese $H_{0}$ e calculando a proporção de simulações em que o teste rejeitou a hipótese nula. A probabilidade do Erro tipo II será estimada gerando um modelo sob a hipótese $H_{1}$ e calculando a proporção de simulações que o teste aceitou a hipótese nula. Neste capítulo não iremos considerar a metodologia de mínimos quadrados ordinários ou ponderado, pois Galea-Rojas et al. (2003) mostraram que os estimadores de máxima verossimilhança do modelo funcional heterocedástico considerado em (2.3) são menos viesados, possuem EQM menores e 
nível de significância empírico bem mais próximo do teórico. Também não iremos considerar o modelo homocedástico apresentado na seção (2.2), pois o enfoque principal deste trabalho são os modelos heterocedásticos. Neste capítulo substituiremos a letra $m$ pela letra $r$ para indicar o índice das repetições. As simulações apresentadas nas seções abaixo foram feitas utilizando o software estatístico R Development Core Team (2006).

\subsection{Réplicas independentes}

\section{Concentrações simétricas - Uniformes}

A fim de comparar os modelos (2.7) e (2.15) das Seções (2.3) e (2.5.1), respectivamente, geramos 2000 simulações de Monte Carlo considerando $x_{i} \sim U(2,40)$, $\kappa_{i}=\left(0,21 x_{i}+0,05\right)^{2}, \lambda_{i}=\left(0,28 E\left(Y_{i}\right)+0,01\right)^{2}, \alpha=0$ e $\beta=1$, o número de concentrações $(n)$ simuladas são $10(i=1,2, \ldots, 10), 15(i=1,2, \ldots, 15)$ e 50 $(i=1,2, \ldots, 50)$ e para cada uma das concentrações replicou-se $15,25,50,100$ e 150 vezes.

Nas Tabelas 3.1 e 3.2 mostramos o comportamento do estimadores de $\alpha$ e $\beta$ usando o modelo (2.15) (Modelo 1) que estima as variâncias via MV e o modelo (2.7) (Modelo 2) que estima as variâncias pelo método dos momentos, lembrando que no Modelo 2 as variâncias são consideradas conhecidas, mas na prática essa suposição não é sempre satisfeita e as variâncias são estimadas usando o método dos momentos . Para cada simulação feita calculamos o EQM, viés e verificamos se o teste $H_{0}:(\alpha, \beta)=(0,1)$ é aceito ou não a $5 \%$ de significância. Como estamos gerando as observações sob a hipótese $H_{0}$ esperamos que, em apenas $5 \%$ das simulações, a hipótese $H_{0}$ seja rejeitada. As formas para as variâncias e concentrações definidas nesta seção seguem a mesma escolha feita por Galea-Rojas et al. (2003) para fins de comparação. 
Tabela 3.1: EQM e Viés estimados (concentração simétrica)

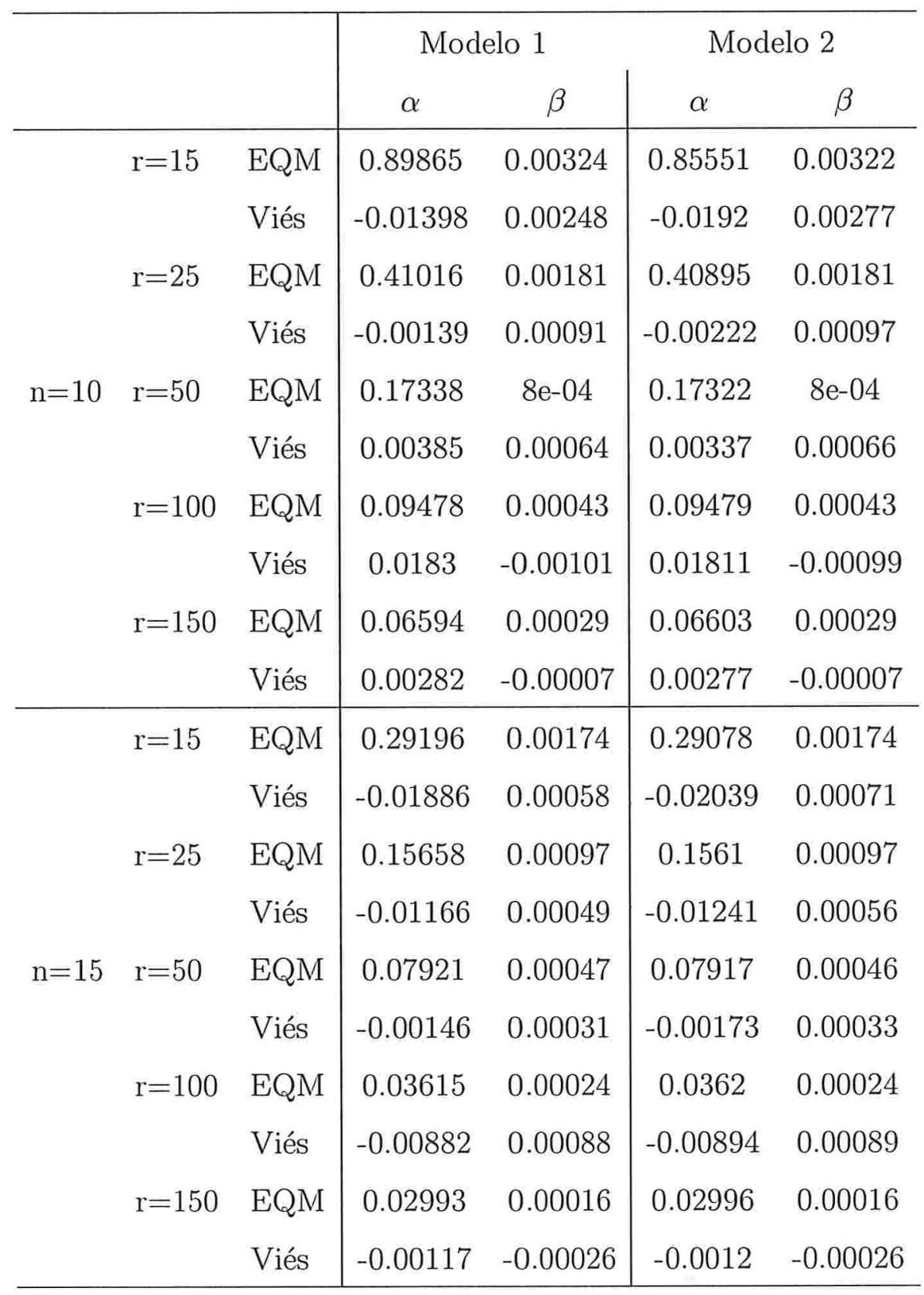


Tabela 3.2: EQM e Viés estimados (concentração simétrica)

\begin{tabular}{|c|c|c|c|c|c|c|}
\hline & & & \multicolumn{2}{|c|}{ Modelo 1} & \multicolumn{2}{|c|}{ Modelo 2} \\
\hline & & & $\alpha$ & $\beta$ & $\alpha$ & $\beta$ \\
\hline \multirow{10}{*}{$\mathrm{n}=50$} & \multirow[t]{2}{*}{$\mathrm{r}=15$} & EQM & 0.03676 & 0.00038 & 0.03652 & 0.00038 \\
\hline & & Viés & 0.00081 & 0.00016 & -0.00126 & 0.00041 \\
\hline & \multirow[t]{2}{*}{$\mathrm{r}=25$} & EQM & 0.02059 & 0.00022 & 0.0207 & 0.00022 \\
\hline & & Viés & 0.00302 & -0.00019 & 0.00252 & -0.00011 \\
\hline & \multirow[t]{2}{*}{$\mathrm{r}=50$} & EQM & 0.01009 & 0.00011 & 0.01011 & 0.00011 \\
\hline & & Viés & -0.00155 & -0.0001 & -0.00178 & -0.00008 \\
\hline & \multirow[t]{2}{*}{$r=100$} & EQM & 0.00561 & 0.00006 & 0.00561 & 0.00006 \\
\hline & & Viés & -0.00078 & 0.00007 & -0.00084 & 0.00008 \\
\hline & \multirow[t]{2}{*}{$\mathrm{r}=150$} & EQM & 0.00335 & 0.00003 & 0.00335 & 0.00003 \\
\hline & & Viés & 0.00182 & -0.00018 & 0.0018 & -0.00018 \\
\hline
\end{tabular}

Nas Tabelas 3.1-3.2 verificamos que o comportamento do erro quadrático médio e do viés tanto no Modelo 1 quanto para o Modelo 2 são semelhantes, não conseguimos afirmar qual deles é o melhor, aparentemente os dois modelos têm o mesmo comportamento. A Figura 3.1 destaca a semelhança dos vieses dos parâmetros de ambos os modelos, a linha pontilhada se refere ao Modelo 1 e a linha tracejada se refere ao Modelo 2. O nível descritivo estimado para os modelos 1 e 2 são obtidos testando os parâmetros sob a hipótese $H_{0}$ (sem viés entre os métodos) a $5 \%$ de significância. 
Figura 3.1: Gráfico do viés dos parâmetros dos Modelos 1 e 2 (concentração simétrica)
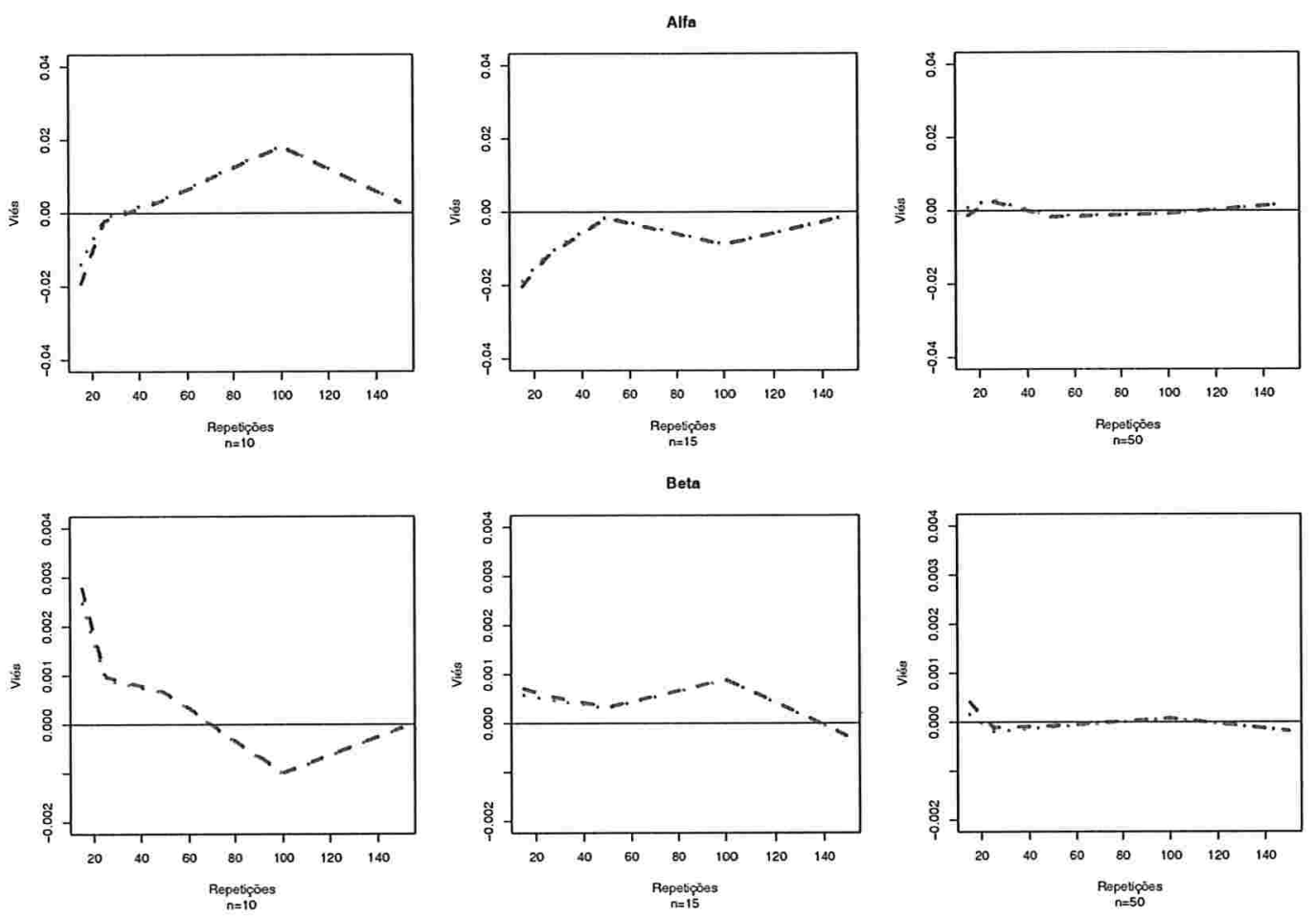
Tabela 3.3: Nível de significância estimado (concentração simétrica)

\begin{tabular}{cc|c|c}
\hline & & Modelo 1 & Modelo 2 \\
\hline $\mathrm{n}=10$ & $\mathrm{r}=50$ & 0.076 & 0.070 \\
& $\mathrm{r}=25$ & 0.067 & 0.065 \\
& $\mathrm{r}=100$ & 0.053 & 0.050 \\
& $\mathrm{r}=150$ & 0.053 & 0.052 \\
\hline $\mathrm{r}=15$ & 0.083 & 0.076 \\
$\mathrm{n}=15$ & $\mathrm{r}=50$ & 0.061 & 0.059 \\
& $\mathrm{r}=100$ & 0.053 & 0.052 \\
$\mathrm{r}=150$ & 0.051 & 0.051 \\
\hline & $\mathrm{r}=15$ & 0.076 & 0.072 \\
$\mathrm{r}=25$ & 0.061 & 0.060 \\
$\mathrm{n}=50$ & $\mathrm{r}=50$ & 0.054 & 0.052 \\
$\mathrm{r}=100$ & 0.056 & 0.057 \\
$\mathrm{r}=150$ & 0.047 & 0.046 \\
\hline
\end{tabular}


Na Tabela 3.3 observamos que, na maioria dos casos, o nível de significância estimado no Modelo 2 se comporta mais próximo do teórico do que no Modelo 1. É importante notar que as variâncias estimadas no Modelo 2 são as variâncias estimadas pelo método dos momentos, ou seja,

$$
\hat{\lambda}_{i}=(1 / r) S_{Y_{i}}^{2}=(1 / r) \sum_{j=1}^{r} \frac{\left(Y_{i j}-\bar{Y}_{i}\right)^{2}}{r-1} \text { e } \hat{\kappa}_{i}=(1 / r) S_{X_{i}}^{2}=(1 / r) \sum_{j=1}^{r} \frac{\left(X_{i j}-\bar{X}_{i}\right)^{2}}{r-1} .
$$

Lembramos que dividimos $S_{Y_{i}}$ por $r$, pois quando utilizamos o Modelo 2 as observações são as médias das réplicas feitas para a $i$-ésima concentração $\left(\bar{Y}_{i}\right)$.

Figura 3.2: Gráfico do nível de significância estimado dos modelos 1 e 2 (concentração simétrica)
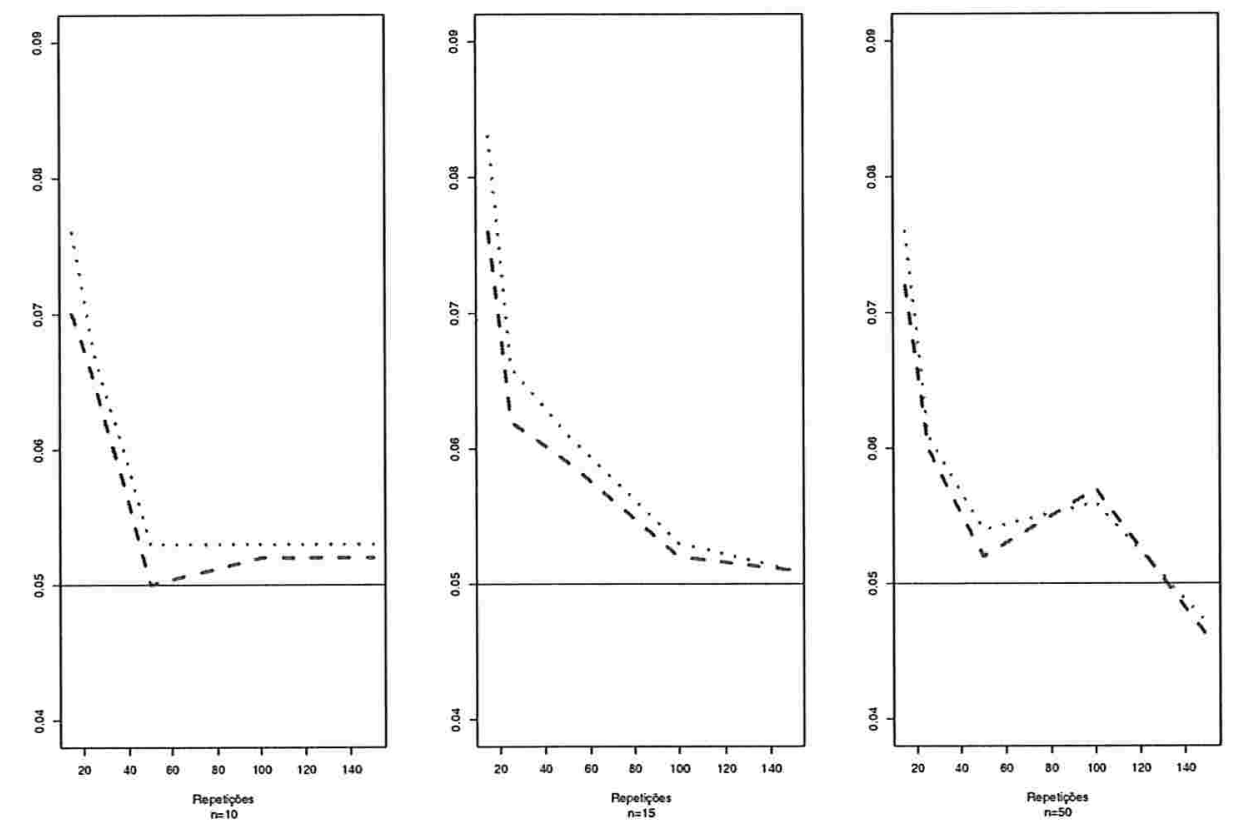

A Figura 3.2 mostra o nível de significância estimado dos Modelos 1 e 2, notase que o Modelo 2 (usual) tem nível de significância mais próximo do teórico que o 
Modelo 1 (proposto). Se corrigirmos as variâncias do Modelo 1 fazendo

$$
\hat{\lambda}_{i}=\sum_{j=1}^{r} \frac{\left(Y_{i j}-\hat{\alpha}-\hat{\beta} \hat{x}_{i}\right)^{2}}{r-3} \text { e } \hat{\kappa}_{i}=\sum_{j=1}^{r} \frac{\left(X_{i j}-\hat{x}_{i}\right)^{2}}{r-1}
$$

teremos resultados mais próximos do esperado. A tabela com os EQM e Viés, usando os estimadores corrigidos para as variâncias, é dada abaixo. 
Tabela 3.4: EQM e Viés estimados, usando o modelo com variâncias corrigidas (concentração simétrica), para $n=10,15$

\begin{tabular}{|c|c|c|c|c|c|c|}
\hline & & & \multicolumn{2}{|c|}{ Modelo 1} & \multicolumn{2}{|c|}{ Modelo 2} \\
\hline & & & $\alpha$ & $\beta$ & $\alpha$ & $\beta$ \\
\hline \multirow{10}{*}{$\mathrm{n}=10$} & \multirow[t]{2}{*}{$\mathrm{r}=15$} & EQM & 0.76831 & 0.00345 & 0.75704 & 0.00342 \\
\hline & & Viés & 0.01065 & -0.00072 & -0.00223 & 0.00041 \\
\hline & \multirow[t]{2}{*}{$\mathrm{r}=25$} & EQM & 0.34243 & 0.00179 & 0.33865 & 0.00178 \\
\hline & & Viés & -0.02153 & 0.00233 & -0.02541 & 0.00267 \\
\hline & \multirow[t]{2}{*}{$\mathrm{r}=50$} & EQM & 0.18377 & 0.00089 & 0.18379 & 0.00089 \\
\hline & & Viés & -0.00762 & 0.00089 & -0.00903 & 0.00101 \\
\hline & \multirow[t]{2}{*}{$\mathrm{r}=100$} & EQM & 0.08479 & 0.00038 & 0.08496 & 0.00038 \\
\hline & & Viés & -0.01071 & 0.00017 & -0.01099 & 0.00019 \\
\hline & \multirow[t]{2}{*}{$\mathrm{r}=150$} & EQM & 0.05911 & 0.0003 & 0.05915 & 0.0003 \\
\hline & & Viés & -0.00319 & 0.00012 & -0.00328 & 0.00013 \\
\hline \multirow{10}{*}{$\mathrm{n}=15$} & \multirow[t]{2}{*}{$\mathrm{r}=15$} & EQM & 0.25795 & 0.00155 & 0.25738 & 0.00155 \\
\hline & & Viés & -0.00186 & -0.00022 & -0.01076 & 0.00072 \\
\hline & \multirow[t]{2}{*}{$\mathrm{r}=25$} & EQM & 0.15608 & 0.00102 & 0.15464 & 0.00102 \\
\hline & & Viés & 0.01864 & -0.00114 & 0.01635 & -0.00083 \\
\hline & \multirow[t]{2}{*}{$\mathrm{r}=50$} & EQM & 0.07659 & 0.00047 & 0.0766 & 0.00047 \\
\hline & & Viés & -0.00087 & 0.00033 & -0.00171 & 0.00042 \\
\hline & \multirow[t]{2}{*}{$\mathrm{r}=100$} & EQM & 0.04651 & 0.00024 & 0.04651 & 0.00024 \\
\hline & & Viés & -0.00238 & 0.00038 & -0.00254 & 0.0004 \\
\hline & \multirow[t]{2}{*}{$\mathrm{r}=150$} & EQM & 0.0229 & 0.00014 & 0.02293 & 0.00014 \\
\hline & & Viés & -0.0033 & 0.0001 & -0.00336 & 0.00011 \\
\hline
\end{tabular}


Tabela 3.5: EQM e Viés estimados, usando o modelo com variâncias corrigidas (concentração simétrica), para $n=50$

\begin{tabular}{|c|c|c|c|c|c|c|}
\hline & & & \multicolumn{2}{|c|}{ Modelo 1} & \multicolumn{2}{|c|}{ Modelo 2} \\
\hline & & & $\alpha$ & $\beta$ & $\alpha$ & $\beta$ \\
\hline \multirow{10}{*}{$\mathrm{n}=50$} & \multirow{2}{*}{$\mathrm{r}=15$} & EQM & 0.03699 & 0.00038 & 0.03681 & 0.00038 \\
\hline & & Viés & 0.00529 & -0.00089 & -0.00139 & -0.00008 \\
\hline & \multirow[t]{2}{*}{$\mathrm{r}=25$} & EQM & 0.02262 & 0.00022 & 0.0227 & 0.00022 \\
\hline & & Viés & 0.00377 & -0.00074 & 0.00129 & -0.00043 \\
\hline & \multirow[t]{2}{*}{$\mathrm{r}=50$} & EQM & 0.00999 & 0.00011 & 0.01 & 0.00011 \\
\hline & & Viés & -0.00291 & 0.0006 & -0.00347 & 0.00068 \\
\hline & \multirow[t]{2}{*}{$\mathrm{r}=100$} & EQM & 0.00543 & 0.00006 & 0.00544 & 0.00006 \\
\hline & & Viés & -0.00037 & 0.00005 & -0.0005 & 0.00007 \\
\hline & \multirow[t]{2}{*}{$r=150$} & EQM & 0.00328 & 0.00003 & 0.00328 & 0.00003 \\
\hline & & Viés & 0.00055 & -0.00012 & 0.00051 & -0.00012 \\
\hline
\end{tabular}

Aparentemente o EQM dos estimadores obtidos com as variâncias corrigidas continuam com o mesmo comportamento dos estimadores obtidos sem a correção. A figura abaixo nos mostra graficamente o comportamento do viés para ambos os modelos, a linha pontilhada refere-se ao Modelo 1 e a linha tracejada refere-se ao Modelo 2. 
Figura 3.3: Gráfico do viés dos parâmetros dos Modelos 1 e 2, usando o modelo com variância corrigida
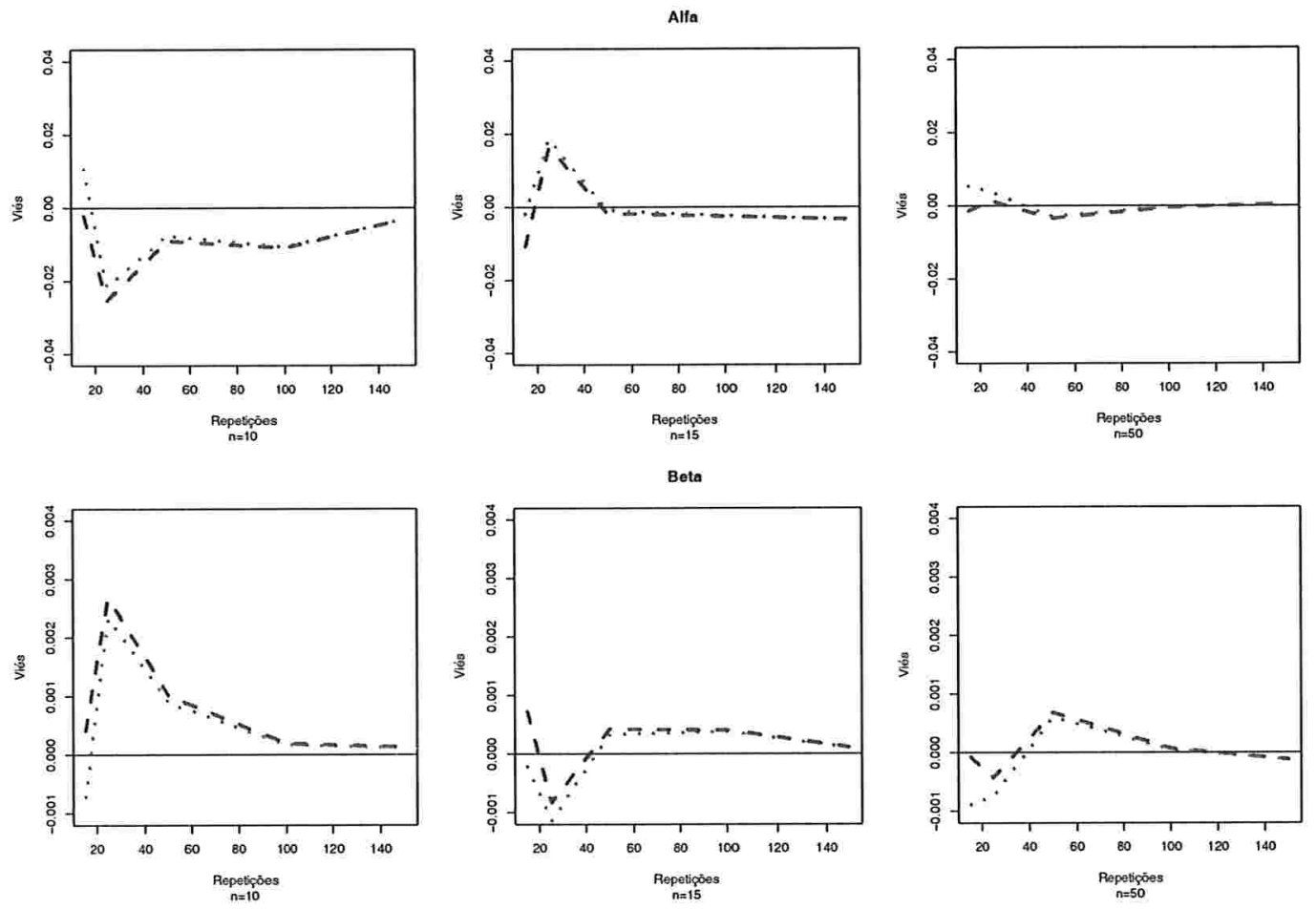

Percebe-se na Figura 3.3 que o viés do Modelo 1 com as variâncias corrigidas é menor que o viés do Modelo 2, quando o número de concentrações é pequena. O comportamento dos dois modelos vai convergindo, desde que o número de réplicas cresça. Abaixo mostramos como se comporta o nível de significância para os modelos em questão. 
Tabela 3.6: Nível de significância estimado, usando o modelo com variâncias corrigidas (concentração simétrica)

\begin{tabular}{cc|c|c}
\hline & & Modelo 1 & Modelo 2 \\
\hline & $\mathrm{r}=15$ & 0.046 & 0.066 \\
$\mathrm{n}=10$ (0) & $\mathrm{r}=50$ & 0.052 & 0.062 \\
& $\mathrm{r}=100$ & 0.049 & 0.051 \\
& $\mathrm{r}=150$ & 0.048 & 0.050 \\
\hline & $\mathrm{r}=15$ & 0.045 & 0.065 \\
$\mathrm{n}=15$ & $\mathrm{r}=50$ & 0.050 & 0.059 \\
& $\mathrm{r}=100$ & 0.047 & 0.052 \\
$\mathrm{r}=150$ & 0.051 & 0.051 \\
\hline & $\mathrm{r}=15$ & 0.048 & 0.066 \\
$\mathrm{r}=25$ & 0.061 & 0.074 \\
$\mathrm{n}=50$ & $\mathrm{r}=50$ & 0.050 & 0.055 \\
$\mathrm{r}=100$ & 0.053 & 0.056 \\
$\mathrm{r}=150$ & 0.046 & 0.048 \\
\hline
\end{tabular}

Com as correções feitas nas variâncias, conseguimos estabilizar o nível de significância para um valor bem próximo do valor teórico no Modelo 1. Não há alterações no Modelo 2 (usual). A figura abaixo evidencia a grande melhoria do nível de significância empírico no Modelo 1 (proposto). 
Figura 3.4: Gráfico do nível de significância estimado dos modelos 1 e 2, usando modelo com variância corrigida (concentração simétrica)
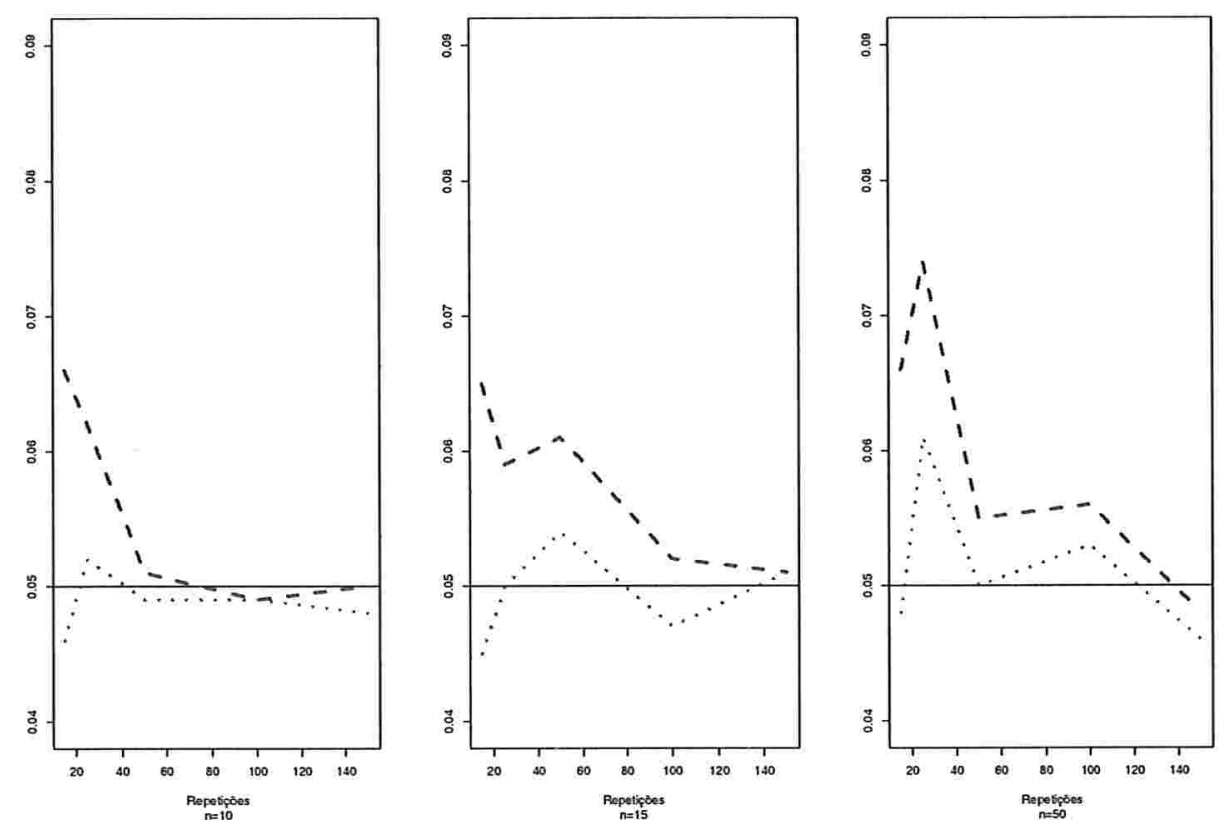

A linha pontilhada se refere ao Modelo 1 (proposto) e a linha tracejada se refere ao Modelo 2 (usual). Os resultados apresentados nesta seção indicam que o Modelo 1 com as variâncias corrigidas deve ser adotado, pois tem menor viés e o nível de significância empírico é muito próximo do teórico, mesmo para amostras pequenas. O Modelo 2 se mostrou pouco confiável para réplicas menores que 25. Todas as comparações a partir deste ponto são feitas usando as variâcnias corrigidas no Modelo 2.

A próxima etapa é estudar o erro tipo II, ou seja, aceitar a hipótese $H_{0}$ sendo ela falsa. O mesmo procedimento para simular as observações é feito, a menos do parâmetro $\beta$. Nas tabelas abaixo mostramos a probabilidade de ocorrência do Erro tipo II quando $\beta$ varia de 1.01 a 1.1 para 10,15 e 50 concentrações e 15, 25, 50, 100 e 150 réplicas por concentração. 
Tabela 3.7: Probabilidade de ocorrer o Erro tipo II nos Modelos 1 e 2, para $n=$ 10, 15, 50 e $r=15,25$ (concentração simétrica)

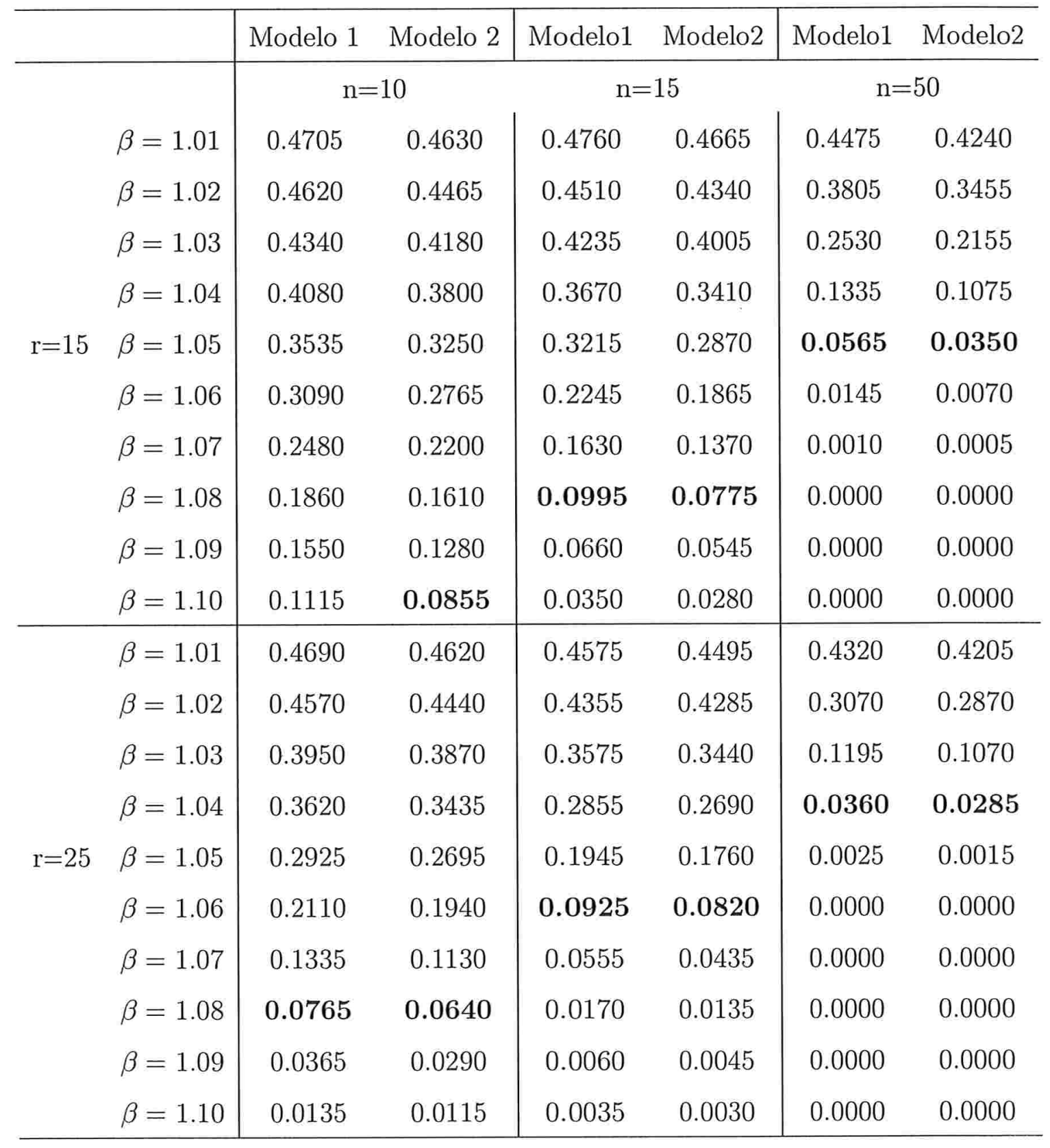


Tabela 3.8: Probabilidade de ocorrer o Erro tipo II nos Modelos 1 e 2, para $n=$ 10,15, 50 e $r=50,100$ (concentração simétrica)

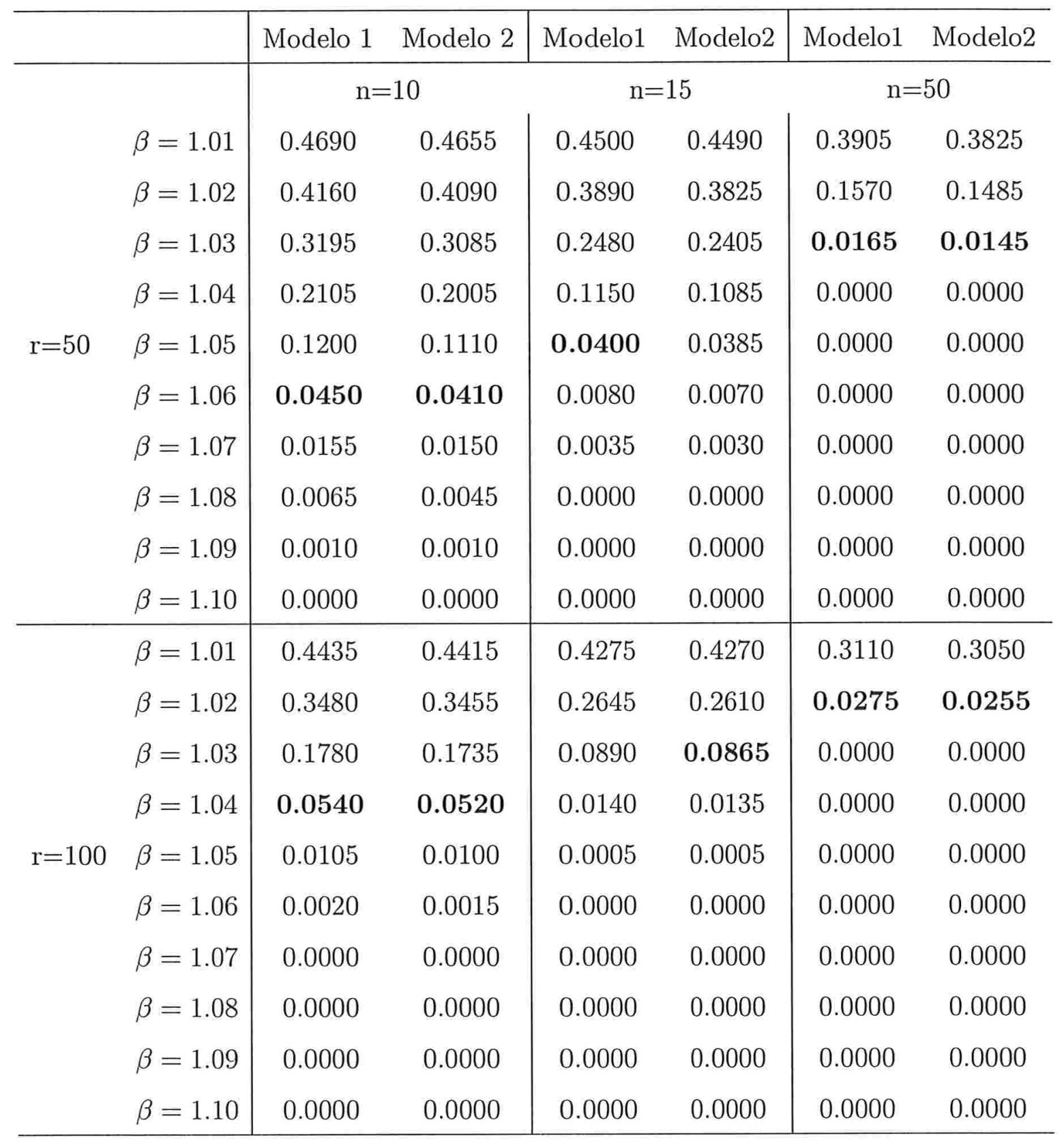


Tabela 3.9: Probabilidade de ocorrer o Erro tipo II nos Modelos 1 e 2, para $n=$ 10, 15, 50 e $r=150$ (concentração simétrica)

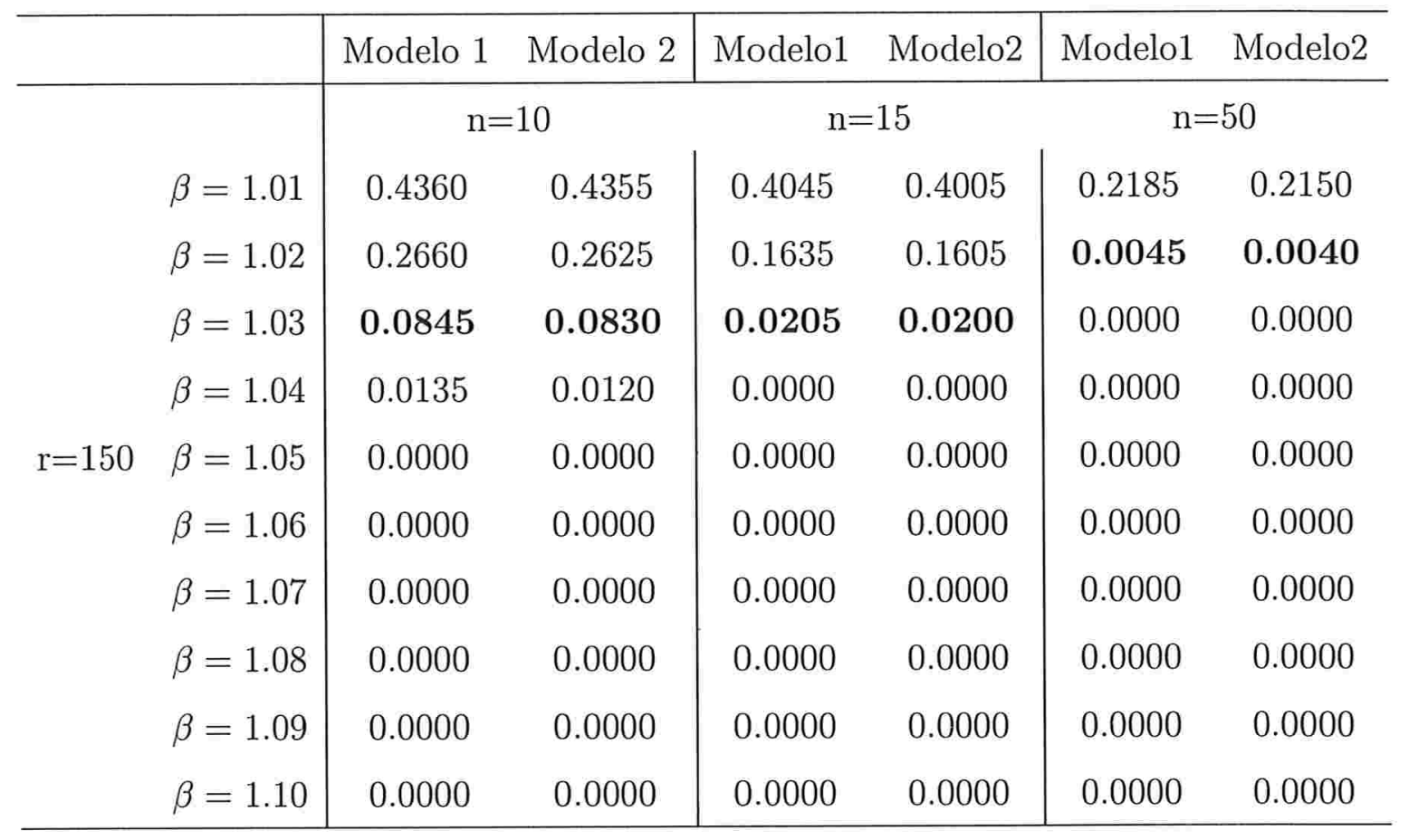

Mostramos nas Tabelas 3.7-3.9, em negrito, o ponto a partir do qual as probabilidades de ocorrência do Erro tipo II estimadas começam a ficar menores do que $10 \%$. As Tabelas 3.7-3.9 nos mostram que o Modelo 2 (usual) tem o poder do teste um pouco maior que o Modelo 1 (proposto). Verificamos também que para 15 concentrações são necessárias 15 réplicas para detectar um $\beta=1.08$, assumindo um erro de 10\%, tanto para o Modelo 1 quanto para o Modelo 2. Para essa combinação de número de concentrações e réplicas aconselhamos usar o Modelo 1, pois a Tabela 3.6 nos mostra que o nível de significância estimado do Modelo 1 é mais próximo do teórico (0.045) que no Modelo 2 (0.065). Note que o poder do teste é afetado pelas diferenças entre os níveis de significância dos Modelos, por isso é importante escolher o Modelo que tem o nível de significância mais próximo do esperado.

Percebemos também que se o número de réplicas e/ou concentrações aumenta 
então o poder do teste aumenta (a probabilidade do Erro tipo II diminui), se o número de concentrações aumenta o poder do teste também aumenta, para os dois Modelos estudados (1 e 2). A figura abaixo nos mostra graficamente esse comportamento.

Figura 3.5: Gráfico do poder do teste dos Modelos 1 e 2, usando modelo com variância corrigida
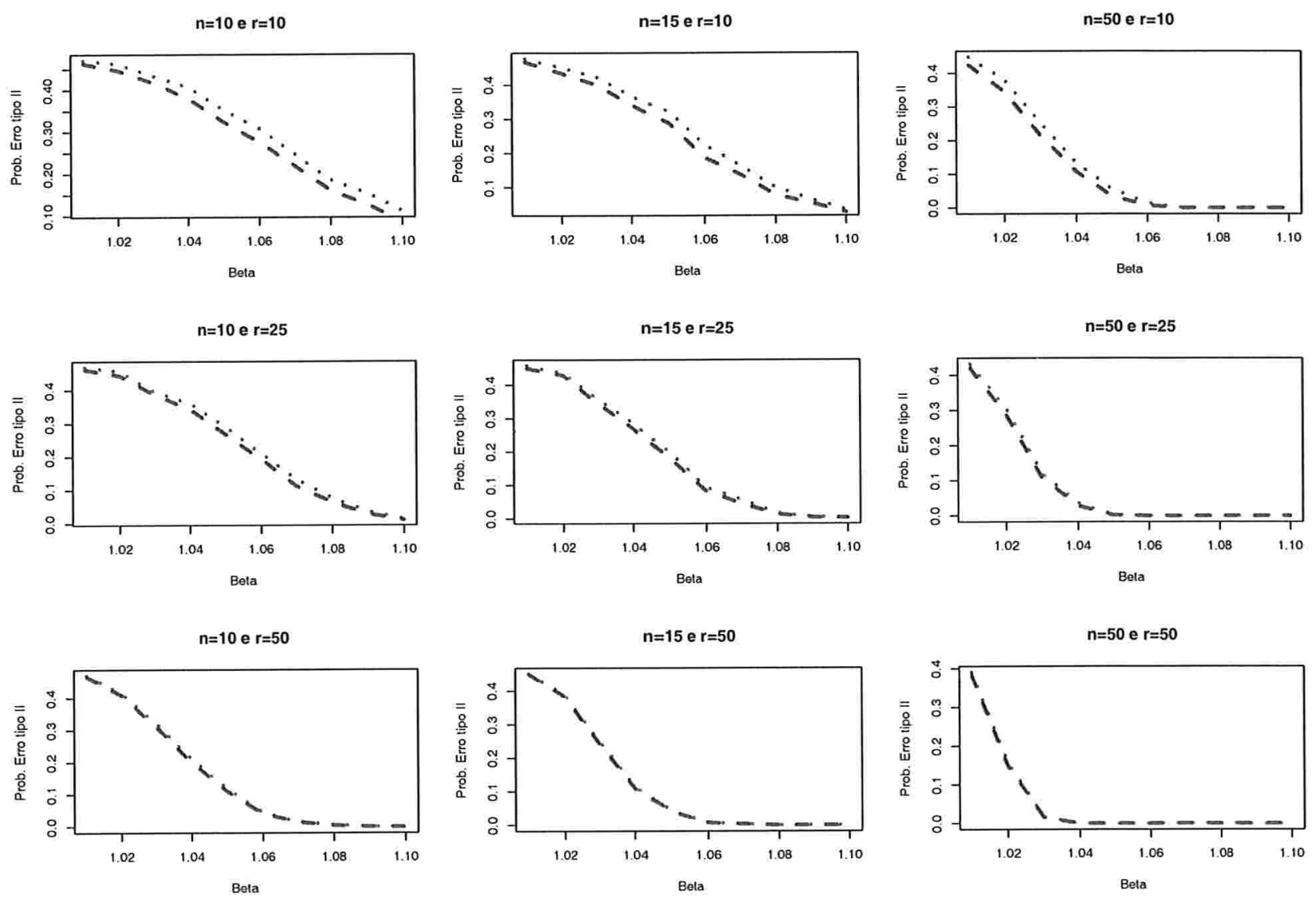
Figura 3.6: Gráfico do poder do teste dos Modelos 1 e 2, usando modelo com variância corrigida
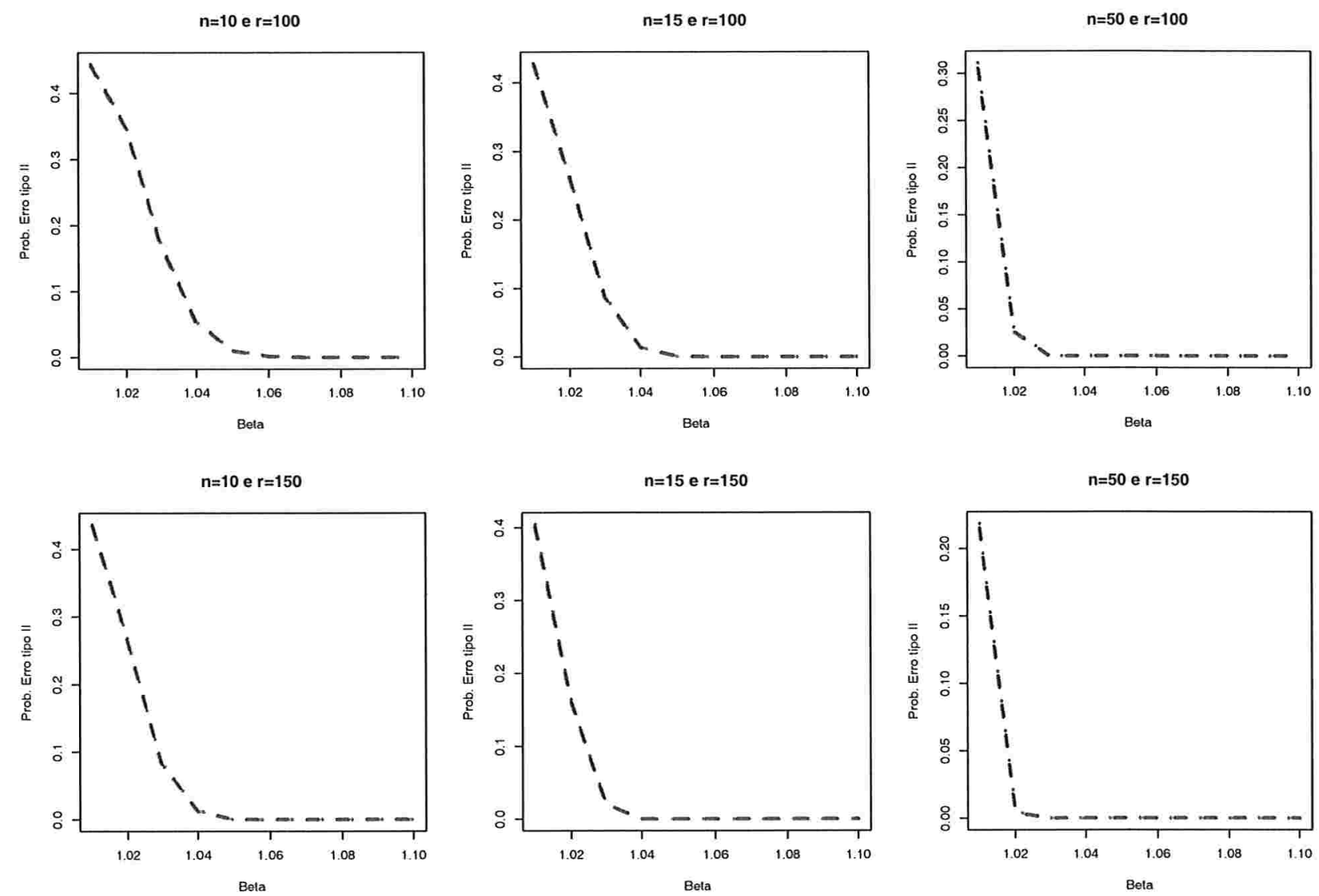

Nas figuras 3.5 e 3.6 notamos que o poder do teste dos modelos vão convergindo. A linha pontilhada se refere ao Modelo 1 e a linha tracejada se refere ao Modelo 2. 
Concentrações assimétricas - Gama

Estudamos o comportamento dos estimadores e testes dos Modelos 1 e 2 quando as concentrações não observadas eram simétricas - uniformes. Nesta etapa do trabalho consideramos que a concentração não observada é assimétrica. É de se esperar que os resultados obtidos sejam parecidos para o caso de concentrações que assumem valores simétricos, pois estamos considerando que as concentrações não observadas são parâmetros do modelo.

Abaixo estão os resultados das simulações de Monte Carlo considerando $x_{i} \sim$ Gama $($ escala $=0.54$, forma $=4.76)$, ou seja, uma distribuição assimétrica para a verdadeira concentração. Os parâmetros neste estágio são iguais aos das simulações quando as concentrações são simétricas - uniformes. A Tabela 3.10 mostra o erro quadrático médio e o viés estimados para os parâmetros do Modelo 1 e do Modelo 2, percebe-se que quando aumentamos o número de réplicas nas concentrações o erro quadrático médio e o viés diminuem, o que é uma característica de estimadores consistentes. Lembramos que as comparações são feitas usando as variâncias corrigidas no Modelo 1. 
Tabela 3.10: EQM e Viés estimados (concentração assimétrica)

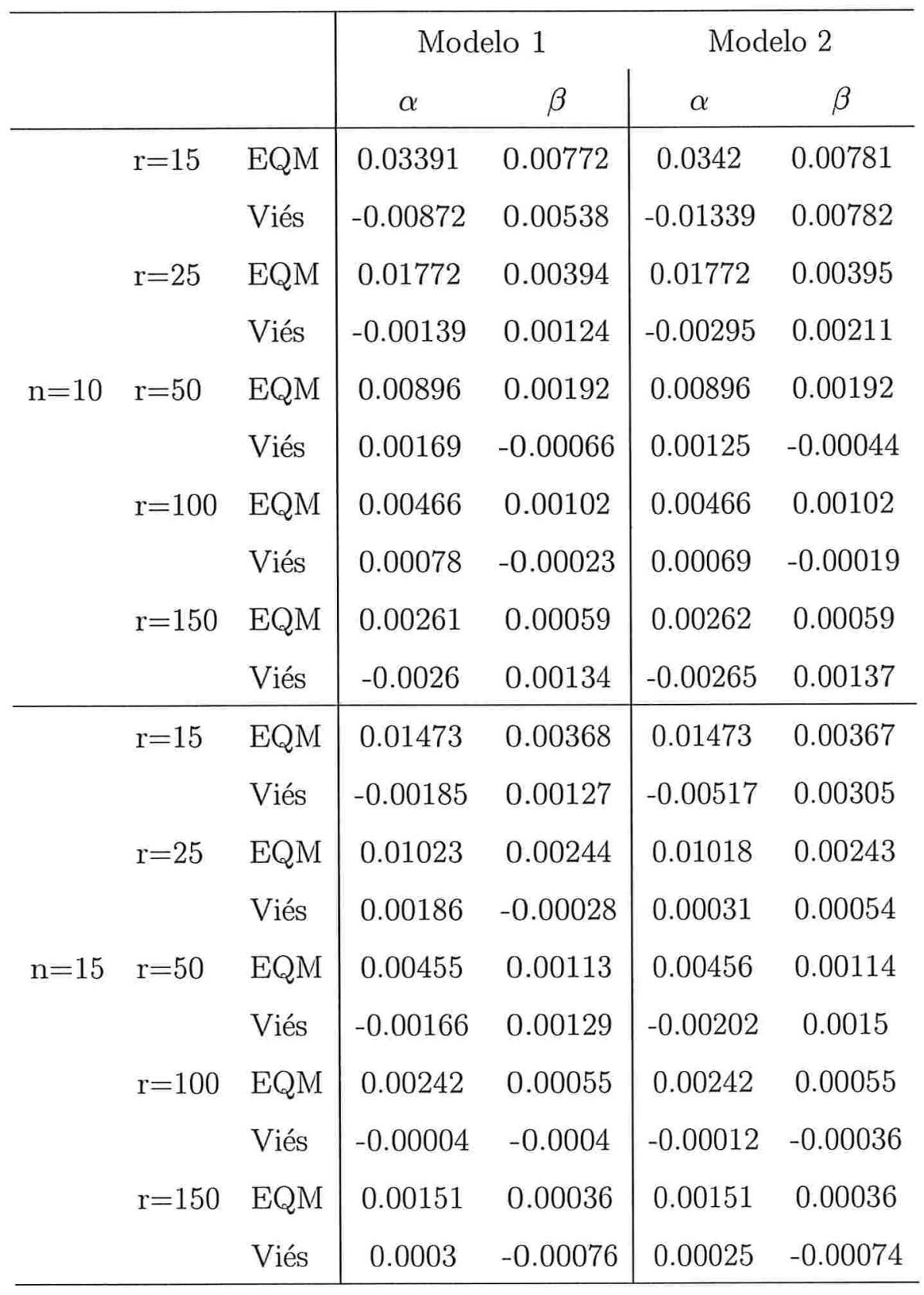


Tabela 3.11: EQM e Viés estimados (concentração assimétrica)

\begin{tabular}{|c|c|c|c|c|c|c|}
\hline & & & \multicolumn{2}{|c|}{ Modelo 1} & \multicolumn{2}{|c|}{ Modelo 2} \\
\hline & & & $\alpha$ & $\beta$ & $\alpha$ & $\beta$ \\
\hline \multirow{10}{*}{$\mathrm{n}=50$} & \multirow{2}{*}{$\mathrm{r}=15$} & EQM & 0.00325 & 0.00091 & 0.00321 & 0.0009 \\
\hline & & Viés & 0.0036 & -0.00174 & 0.00072 & 0.00002 \\
\hline & \multirow[t]{2}{*}{$\mathrm{r}=25$} & EQM & 0.00196 & 0.00053 & 0.00195 & 0.00053 \\
\hline & & Viés & 0.00196 & -0.00132 & 0.00087 & -0.00068 \\
\hline & \multirow[t]{2}{*}{$\mathrm{r}=50$} & EQM & 0.00087 & 0.00024 & 0.00087 & 0.00024 \\
\hline & & Viés & 0.00075 & -0.00036 & 0.00048 & -0.00021 \\
\hline & \multirow[t]{2}{*}{$\mathrm{r}=100$} & EQM & 0.00047 & 0.00013 & 0.00047 & 0.00013 \\
\hline & & Viés & -0.00009 & -0.00024 & -0.00015 & -0.0002 \\
\hline & \multirow[t]{2}{*}{$\mathrm{r}=150$} & EQM & 0.00031 & 0.00009 & 0.00031 & 0.00009 \\
\hline & & Viés & -0.00001 & 0.00019 & -0.00004 & 0.00021 \\
\hline
\end{tabular}

As Tabelas 3.10-3.11 mostram que para as concentrações assimétricas o erro quadrático médio e o viés dos estimadores diminuem conforme o número de réplicas aumenta. Não é possível afirmar qual dos Modelos se comporta melhor, pois em alguns casos o Modelo 1 se comporta melhor que o Modelo 2 e em outros casos o Modelo 2 se comporta melhor que o Modelo 1. A figura 3.7 mostra o viés empírico das estimativas dos parâmetros para uma comparação gráfica desse efeito. 
Figura 3.7: Gráfico do viés dos parâmetros dos Modelos 1 e 2, usando o modelo com variância corrigida (concentração assimétrica)
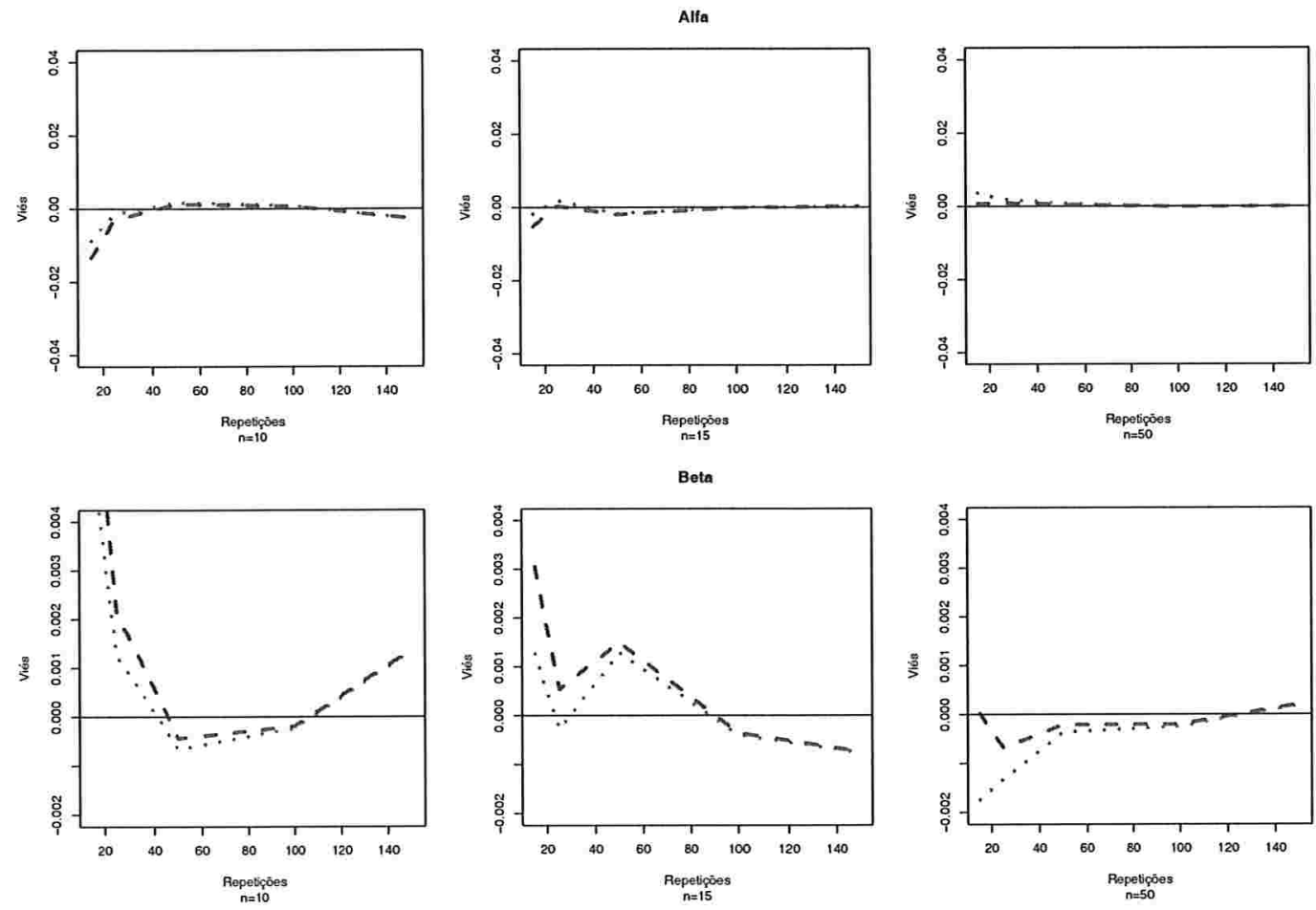

A Figura 3.7 mostra graficamente o comportamento do viés, visto na Tabela 3.10. A linha pontilhada indica o viés para os parâmetros do Modelo 1 e a linha tracejada indica o viés para os parâmetros do Modelo 2. Verificamos claramente que, quando o número de repetições é pequeno e $n=10,15$, o Modelo 1 estima ambos parâmetros com menos viés que o Modelo 2, já quando $n=50$ o Modelo 2 mensura com menos viés. A explicação para esse fato é que no Modelo 1 a teoria assintótica se baseia no número de repetições, portanto se o número de concentrações aumenta a instabilidade na estimação dos parâmetros também aumenta, já no Modelo 2 a teoria assintótica se baseia no número de concentrações, dessa forma quanto maior o número de concentrações mais estável e precisa será a estimação. 
Tabela 3.12: Nível de significância estimado (concentração assimétrica)

\begin{tabular}{cc|c|c}
\hline & & Modelo 1 & Modelo 2 \\
\hline & $\mathrm{r}=15$ & 0.054 & 0.069 \\
$\mathrm{n}=10$ & $\mathrm{r}=50$ & 0.046 & 0.058 \\
& $\mathrm{r}=100$ & 0.049 & 0.052 \\
& $\mathrm{r}=150$ & 0.048 & 0.060 \\
& $\mathrm{r}=15$ & 0.058 & 0.081 \\
$\mathrm{n}=15$ & $\mathrm{r}=50$ & 0.057 & 0.063 \\
& $\mathrm{r}=100$ & 0.052 & 0.055 \\
$\mathrm{r}=150$ & 0.055 & 0.057 \\
\hline & $\mathrm{r}=15$ & 0.054 & 0.077 \\
$\mathrm{n}=50$ & $\mathrm{r}=50$ & 0.048 & 0.049 \\
$\mathrm{r}=100$ & 0.053 & 0.056 \\
$\mathrm{r}=150$ & 0.048 & 0.050 \\
\hline
\end{tabular}

Notamos na Tabela 3.12 que o Modelo 1 se comporta de forma mais adequada que o Modelo 2, mesmo para repetições pequenas e grande número de concentrações. Com isso concluímos que para concentrações simétricas e assimétricas o Modelo 1, que considera as repetições no processo de estimação, se comporta mais próximo do esperado do que o Modelo 2. A figura abaixo mostra a Tabela 3.12 graficamente. 
Figura 3.8: Gráfico do nível de significância estimado dos modelos 1 e 2, usando modelo com variância corrigida (concentração assimétrica)
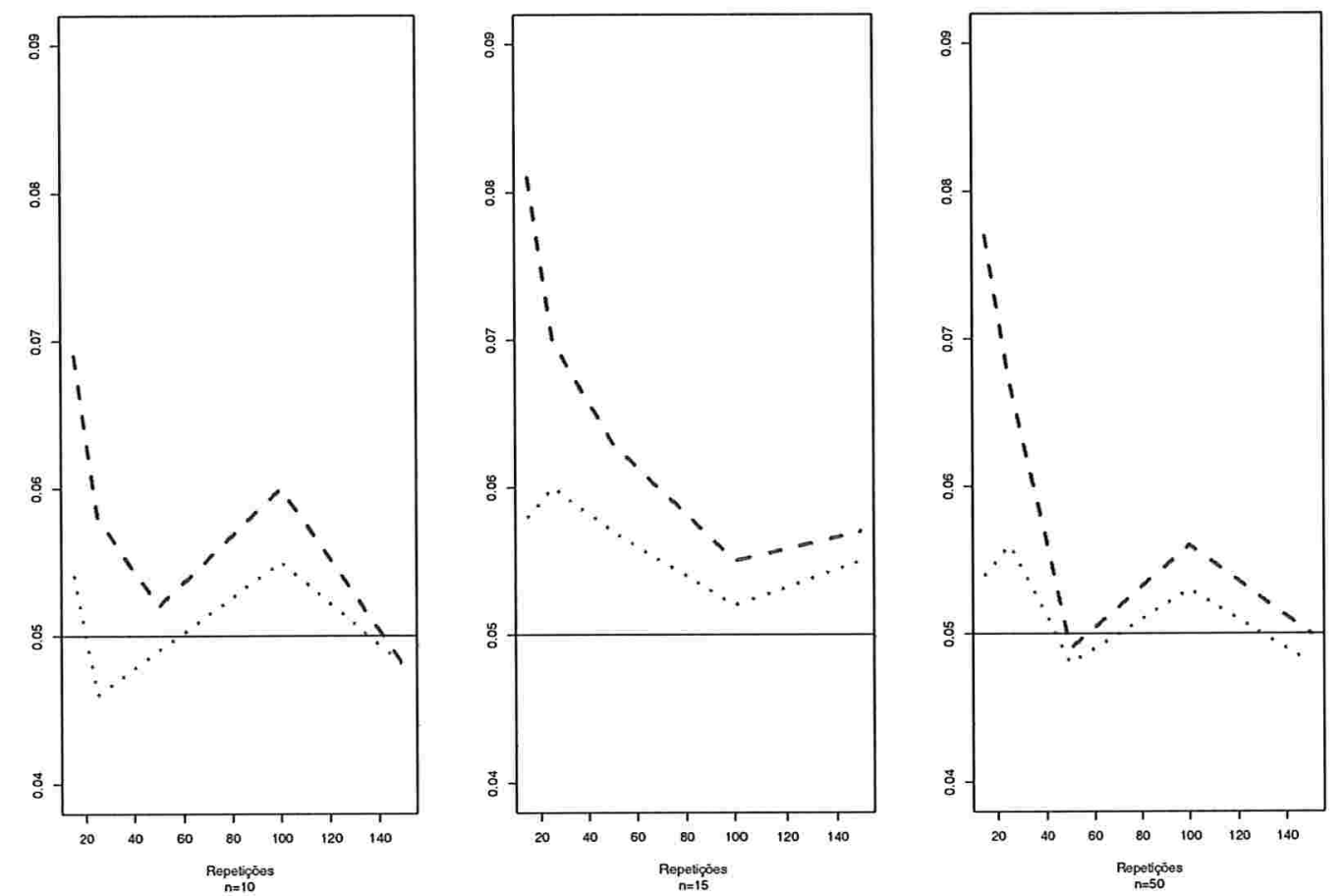

A Figura 3.8 mostra o quão melhor é o Modelo 1 (proposto) em relação ao Modelo 2 (usual). A linha pontilhada se refere ao Modelo 1 e a linha tracejada se refere ao Modelo 2. Fazemos um estudo do poder do teste para cada modelo, da mesma forma que foi feita no caso em que as concentrações eram simétricas, as tabelas abaixo mostram os resultados. 
Tabela 3.13: Probabilidade de ocorrer o Erro tipo II nos modelos 1 e 2, para $n=$ 10, 15, 50 e $r=15,25$ (concentração assimétrica)

\begin{tabular}{|c|c|c|c|c|c|c|c|}
\hline & & $\begin{array}{r}\text { Modelo } 1 \\
n=\end{array}$ & $\begin{array}{l}\text { Modelo } 2 \\
0\end{array}$ & $\begin{array}{r}\text { Modelo1 } \\
\qquad \mathrm{n}=\end{array}$ & $\begin{array}{l}\text { Modelo2 } \\
15\end{array}$ & Modelo1 & $\begin{array}{l}\text { Modelo2 } \\
50\end{array}$ \\
\hline & $\beta=1.01$ & 0.4720 & 0.4660 & 0.4705 & 0.4560 & 0.4490 & 0.4285 \\
\hline & $\beta=1.02$ & 0.4575 & 0.4425 & 0.4655 & 0.4490 & 0.3735 & 0.3430 \\
\hline & $\beta=1.03$ & 0.4440 & 0.4300 & 0.4225 & 0.4030 & 0.2685 & 0.2375 \\
\hline & $\beta=1.04$ & 0.4135 & 0.3845 & 0.3645 & 0.3415 & 0.1550 & 0.1225 \\
\hline $\mathrm{r}=15$ & $\beta=1.05$ & 0.3685 & 0.3450 & 0.3125 & 0.2760 & 0.0585 & 0.0410 \\
\hline & $\beta=1.06$ & 0.3330 & 0.3015 & 0.2640 & 0.2295 & 0.0210 & 0.0125 \\
\hline & $\beta=1.07$ & 0.2665 & 0.2410 & 0.1890 & 0.1535 & 0.0040 & 0.0030 \\
\hline & $\beta=1.08$ & 0.2225 & 0.1965 & 0.1185 & 0.0915 & 0.0010 & 0.0005 \\
\hline & $\beta=1.09$ & 0.1740 & 0.1400 & 0.0725 & 0.0620 & 0.0000 & 0.0000 \\
\hline & $\beta=1.10$ & 0.1245 & 0.1005 & 0.0445 & 0.0310 & 0.0000 & 0.0000 \\
\hline & $\beta=1.01$ & 0.4730 & 0.4660 & 0.4635 & 0.4570 & 0.4330 & 0.4210 \\
\hline & $\beta=1.02$ & 0.4485 & 0.4430 & 0.4350 & 0.4230 & 0.3025 & 0.2845 \\
\hline & $\beta=1.03$ & 0.4220 & 0.4070 & 0.3805 & 0.3700 & 0.1615 & 0.1480 \\
\hline & $\beta=1.04$ & 0.3575 & 0.3430 & 0.3115 & 0.2920 & 0.0460 & 0.03650 \\
\hline$r=25$ & $\beta=1.05$ & 0.2860 & 0.2705 & 0.2110 & 0.1920 & 0.0070 & 0.0055 \\
\hline & $\beta=1.06$ & 0.2130 & 0.1980 & 0.1270 & 0.1130 & 0.0005 & 0.0005 \\
\hline & $\beta=1.07$ & 0.1415 & 0.1290 & 0.0650 & 0.0565 & 0 & 0 \\
\hline & $\beta=1.08$ & 0.0920 & 0.0785 & 0.0245 & 0.0180 & 0 & 0 \\
\hline & $\beta=1.09$ & 0.0510 & 0.0445 & 0.0100 & 0.0100 & 0 & 0 \\
\hline & $\beta=1.10$ & 0.0260 & 0.0215 & 0.0055 & 0.0030 & 0 & 0 \\
\hline
\end{tabular}


Tabela 3.14: Probabilidade de ocorrer o Erro tipo II nos modelos 1 e 2, para $n=$ 10, 15, 50 e $r=50,100$ (concentração assimétrica)

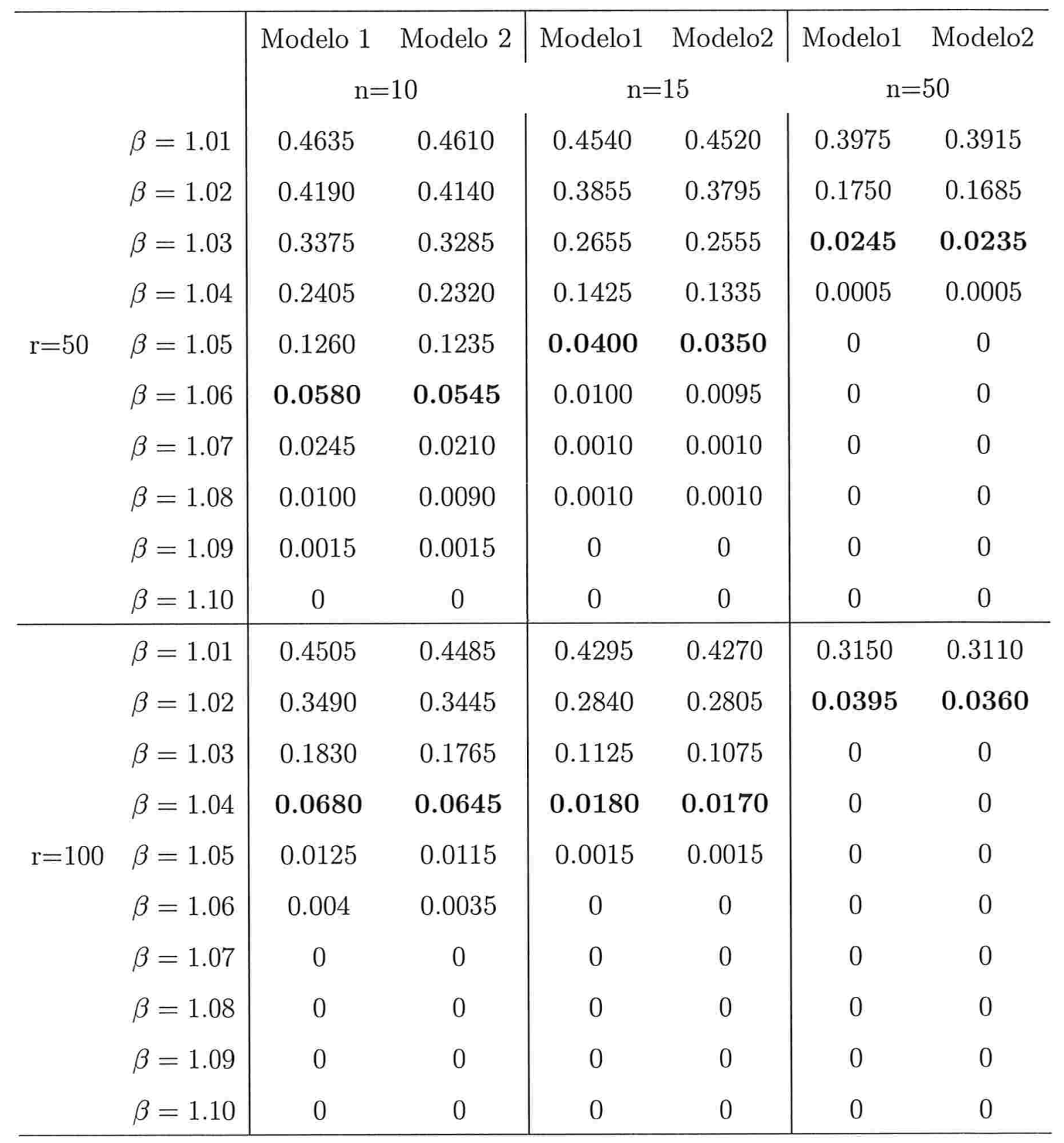


Tabela 3.15: Probabilidade de ocorrer o Erro tipo II nos modelos 1 e 2, para $n=$ $10,15,50$ e $r=150$ (concentração assimétrica)

\begin{tabular}{r|cc|cc|ccc}
\hline & Modelo 1 & Modelo 2 & Modelo1 & Modelo2 & Modelo1 & Modelo2 \\
& \multicolumn{2}{|c|}{$\mathrm{n}=10$} & \multicolumn{2}{|c}{$\mathrm{n}=15$} & \multicolumn{2}{c}{$\mathrm{n}=50$} \\
$\beta=1.01$ & 0.4400 & 0.4395 & 0.4105 & 0.4090 & 0.2440 & 0.2425 \\
$\beta=1.02$ & 0.2900 & 0.2870 & 0.1945 & 0.1930 & $\mathbf{0 . 0 0 4 5}$ & $\mathbf{0 . 0 0 4 5}$ \\
$\beta=1.03$ & 0.104 & 0.1020 & $\mathbf{0 . 0 2 9 0}$ & $\mathbf{0 . 0 2 8 5}$ & 0 & 0 \\
$\beta=1.04$ & 0.020 & $\mathbf{0 . 0 1 9 5}$ & 0.0010 & 0.0005 & 0 & 0 \\
$\mathrm{r}=150$ & $\beta=1.05$ & 0.0020 & 0.0020 & 0 & 0 & 0 & 0 \\
$\beta=1.06$ & 0 & 0 & 0 & 0 & 0 & 0 \\
$\beta=1.07$ & 0 & 0 & 0 & 0 & 0 & 0 \\
$\beta=1.08$ & 0 & 0 & 0 & 0 & 0 & 0 \\
$\beta=1.09$ & 0 & 0 & 0 & 0 & 0 & 0 \\
$\beta=1.10$ & 0 & 0 & 0 & 0 & 0 & 0 \\
\hline
\end{tabular}

Mostramos nas Tabelas 3.13-3.15, em negrito, o ponto a partir do qual as probabilidades de ocorrência do Erro tipo II estimadas começam a ficar menores do que $10 \%$. Percebe-se que com o aumento das réplicas e/ou das concentrações os testes ficam mais poderosos. Para a configuração $n=10$ e $r=15$ é necessário aumentar o valor de $\beta$ para saber qual o ponto que o teste começa a rejeitar a hipótese $H_{0}$ tal que a probabilidade do Erro tipo II seja menor que 10\%, é bem provável que para $\beta=1.12$ a probabilidade desse erro seja satisfatória. Fixando o $n=10$, ou seja, o número de concentrações igual a 10, teremos que, para detectar um $\beta=1.04$ será necessário replicar aproximadamente 100 vezes cada concentração, no entanto para detectar um $\beta=1.08$ é preciso replicar 25 vezes cada concentração. A figura abaixo mostra o efeito do aumento das réplicas e concentrações no poder do teste. 
Figura 3.9: Gráfico do poder do teste dos modelos 1 e 2, (concentração assimétrica)
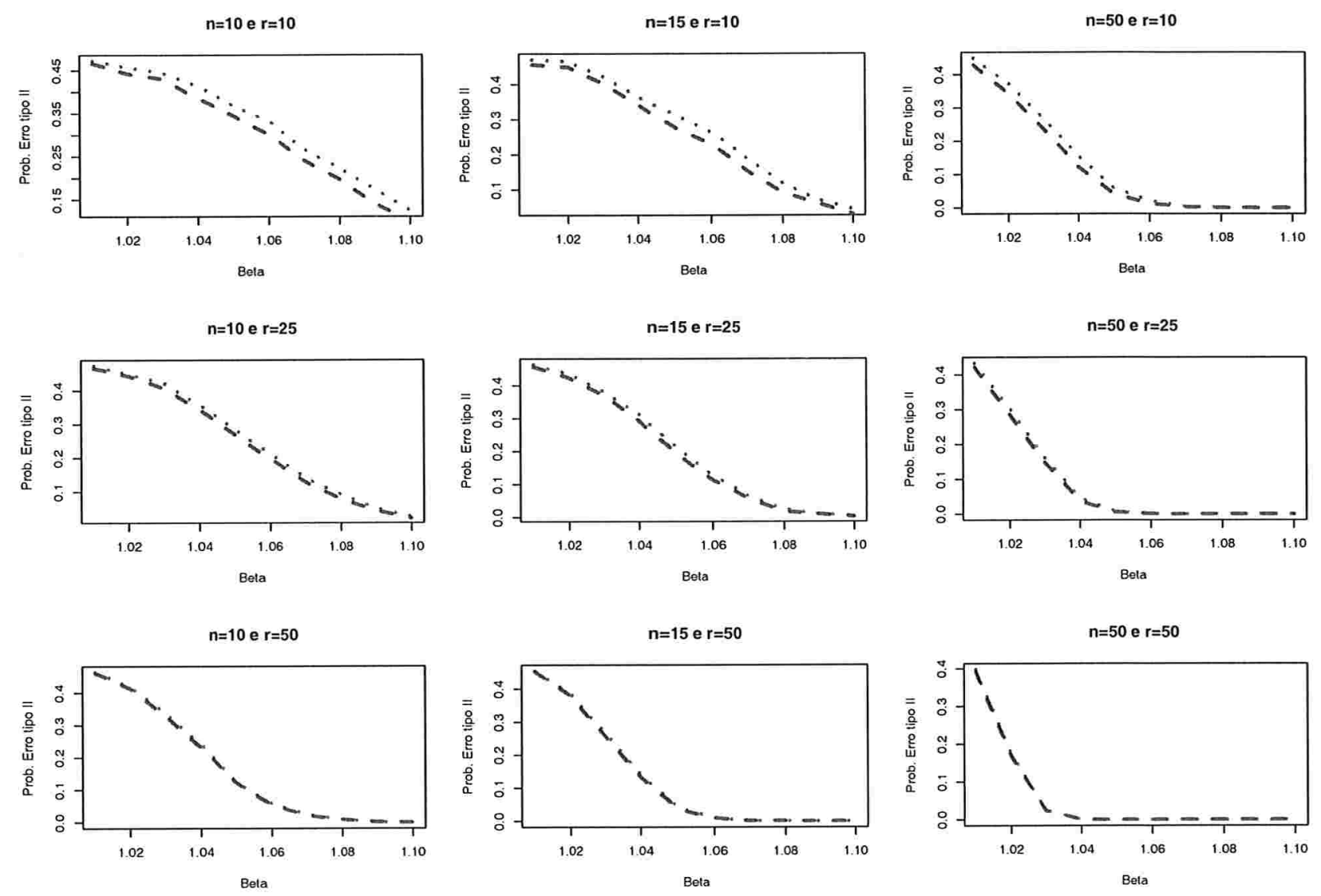
Figura 3.10: Gráfico do poder do teste dos modelos 1 e 2, (concentração assimétrica)
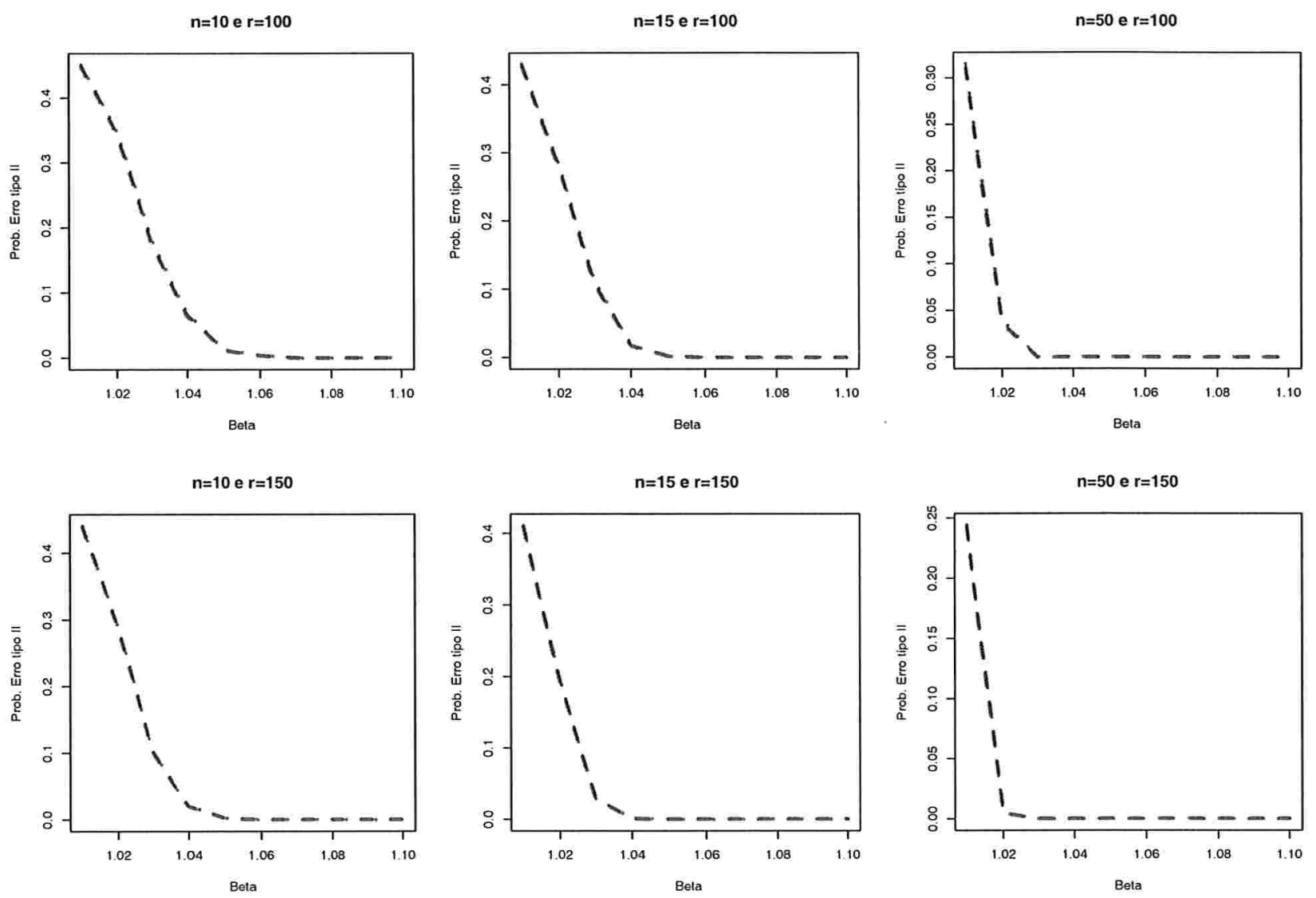
A linha pontilhada se refere ao Modelo 1 (proposto) e a linha tracejada ao Modelo 2 (usual).

\subsection{Réplicas dependentes}

\section{concentração simétrica - uniforme}

Nesta seção iremos considerar repetições dependentes, com a estrutura de correlação autoregressiva de ordem 1. Foram geradas 2000 simulações de Monte carlo considerando $x_{i} \sim U(2,40), \kappa_{i}=\left(0,21 x_{i}+0,05\right)^{2}, \lambda_{i}=\left(0,28 E\left(Y_{i}\right)+0,01\right)^{2}$, $\rho_{1}=0.3, \rho_{2}=0.2, \alpha=0$ e $\beta=1$, o número de concentrações simuladas são 10 $(i=1,2, \ldots, 10), 15(i=1,2, \ldots, 15)$ e $50(i=1,2, \ldots, 50)$ e para cada uma das concentrações replicou-se 15, 25, 50, 100 e 150 vezes. Manteremos a mesma terminologia para os Modelos, o modelo dado em (2.15) (Modelo 1) que estima as variâncias via MV, o modelo dado em (2.7) (Modelo 2) que estima as variâncias pelo método dos momentos e o modelo dado em (2.21) (Modelo 3) que considera a estrutura de dependência autoregressiva para as repetições. Aqui estamos interessados em verificar o comportamento dos modelos quando uma estrutura de correlação simples é imposta nas repetições de cada concentração. As tabelas 3.16 e 3.17 nos retornam o EQM e vieses empíricos dos estimadores dos três modelos. 
Tabela 3.16: EQM e Viés estimados para os Modelos 1, 2 e 3 (concentração simétrica)

\begin{tabular}{|c|c|c|c|c|c|c|c|c|}
\hline & & & \multicolumn{2}{|c|}{ Modelo 1} & \multicolumn{2}{|c|}{ Modelo 2} & \multicolumn{2}{|c|}{ Modelo 3} \\
\hline & & & $\alpha$ & $\beta$ & $\alpha$ & $\beta$ & $\alpha$ & $\beta$ \\
\hline \multirow{10}{*}{$\mathrm{n}=10$} & \multirow[t]{2}{*}{$\mathrm{r}=15$} & EQM & 0.88688 & 0.00504 & 0.89834 & 0.00509 & 0.86996 & 0.00497 \\
\hline & & Viés & 0.03247 & -0.00158 & 0.01501 & 0.00034 & 0.04131 & -0.00217 \\
\hline & \multirow{2}{*}{$\mathrm{r}=25$} & EQM & 0.70200 & 0.00309 & 0.69407 & 0.00308 & 0.68731 & 0.00305 \\
\hline & & Viés & 0.03582 & -0.00154 & 0.02573 & -0.0007 & 0.04301 & -0.00245 \\
\hline & \multirow[t]{2}{*}{$\mathrm{r}=50$} & EQM & 0.31342 & 0.00151 & 0.31113 & 0.0015 & 0.31205 & 0.0015 \\
\hline & & Viés & -0.01062 & 0.00042 & -0.0124 & 0.00059 & -0.00454 & -0.00006 \\
\hline & \multirow[t]{2}{*}{$\mathrm{r}=100$} & EQM & 0.15834 & 0.00078 & 0.15817 & 0.00078 & 0.15802 & 0.00078 \\
\hline & & Viés & 0.01553 & -0.00056 & 0.01492 & -0.00052 & 0.0184 & -0.00078 \\
\hline & \multirow[t]{2}{*}{$\mathrm{r}=150$} & EQM & 0.10244 & 0.00046 & 0.10258 & 0.00046 & 0.1024 & 0.00046 \\
\hline & & Viés & -0.00937 & 0.00058 & -0.00946 & 0.0006 & -0.00683 & 0.00037 \\
\hline \multirow{10}{*}{$\mathrm{n}=15$} & \multirow[t]{2}{*}{$\mathrm{r}=15$} & EQM & 0.41385 & 0.00258 & 0.41357 & 0.00262 & 0.41959 & 0.00257 \\
\hline & & Viés & -0.02974 & 0.00323 & -0.04802 & 0.00522 & -0.02088 & 0.0027 \\
\hline & \multirow[t]{2}{*}{$\mathrm{r}=25$} & EQM & 0.23157 & & 0.23313 & 0.00149 & 0.23099 & 0.00148 \\
\hline & & Viés & 0.00366 & -0.00029 & -0.00568 & 0.00059 & 0.00784 & -0.00087 \\
\hline & \multirow[t]{2}{*}{$\mathrm{r}=50$} & EQM & 0.12669 & 0.0008 & 0.12688 & 0.0008 & 0.12588 & 0.0008 \\
\hline & & Viés & 0.00034 & -0.00074 & -0.00053 & -0.0006 & 0.00498 & -0.00125 \\
\hline & \multirow[t]{2}{*}{$\mathrm{r}=100$} & EQM & 0.06412 & 0.00039 & 0.06424 & 0.0004 & 0.06498 & 0.00039 \\
\hline & & Viés & -0.01747 & 0.00193 & -0.01795 & 0.00198 & -0.01544 & 0.00169 \\
\hline & \multirow[t]{2}{*}{$\mathrm{r}=150$} & EQM & 0.05196 & 0.00028 & 0.05201 & 0.00028 & 0.05189 & 0.00028 \\
\hline & & Viés & -0.00625 & 0.00031 & -0.00629 & 0.00031 & -0.00435 & 0.00014 \\
\hline
\end{tabular}


Tabela 3.17: EQM e Viés estimados para os Modelos 1, 2 e 3 (concentração simétrica)

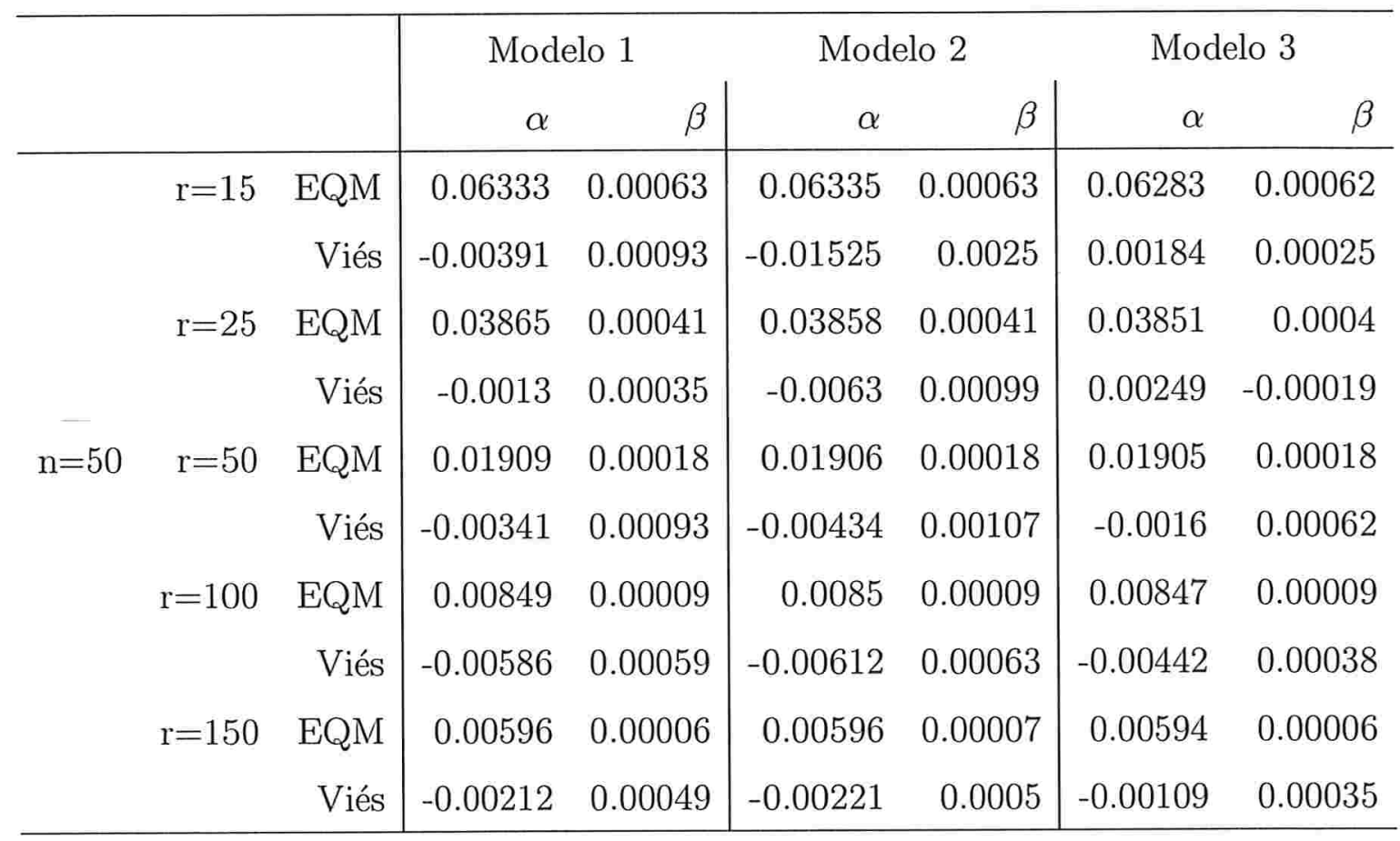


As tabelas acima nos mostram que o EQM do Modelo 3 é sempre inferior que os outros dois modelos, a menos das combinações $(n=15$ e $r=15)$ e $(n=15$ e $r=100$ ). A figura 3.11 nos mostra graficamente o comportamento dos vieses em cada modelo.

Figura 3.11: Gráfico do viés dos parâmetros dos Modelos 1, 2 e 3 (concentração simétrica)
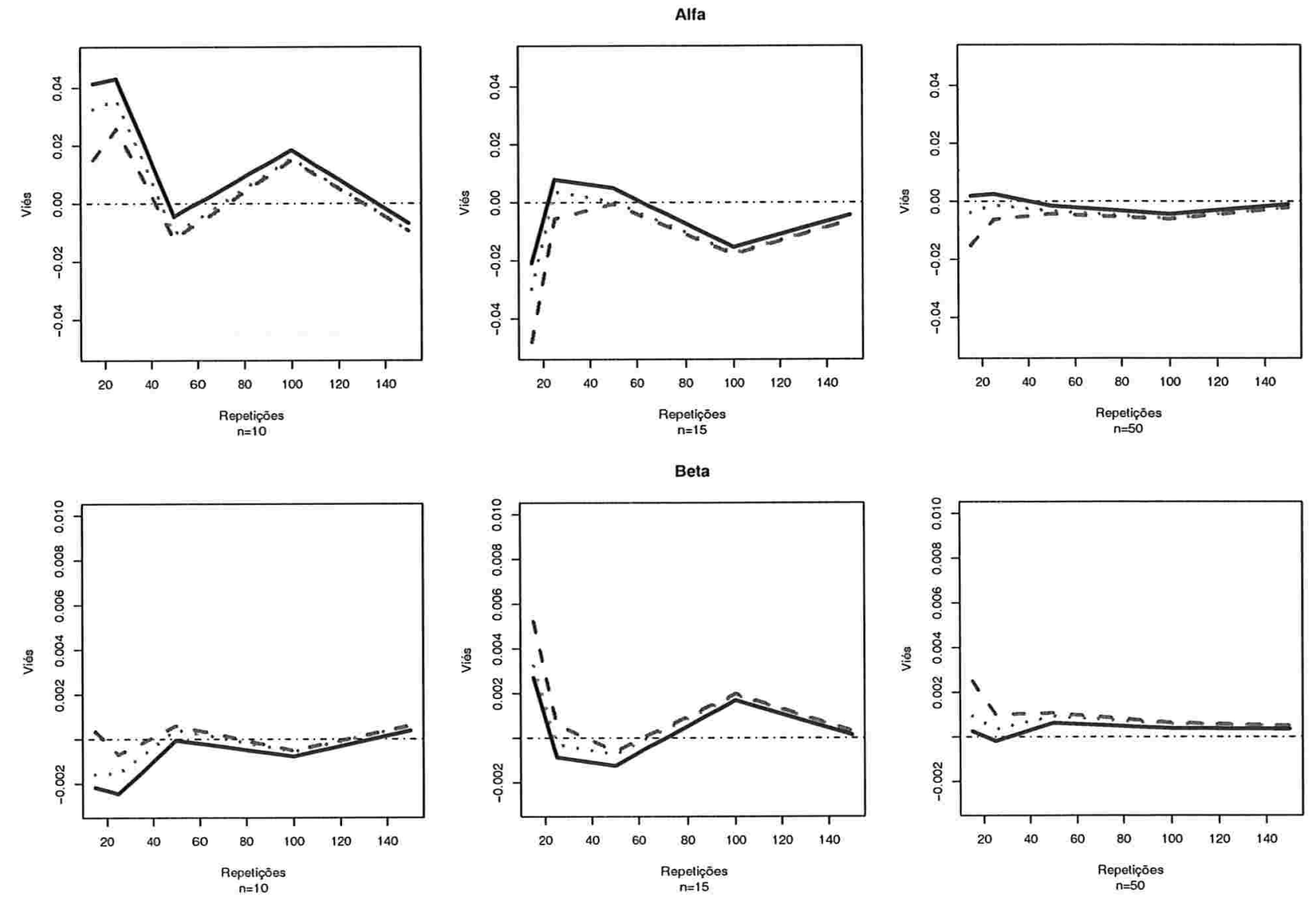

A linha pontilhada se refere ao Modelo 1, a linha tracejada ao Modelo 2 e a linha cheia ao Modelo 3. Pela Figura 3.11 notamos que no caso de 10 concentrações, os parâmetros estimados do Modelo 3 possuem mais viés que os parâmetros estimados 
dos Modelos 1 e 2. Abaixo a tabela do nível de significância estimado.

Tabela 3.18: Nível de significância estimado para os Modelos 1, 2 e 3 (concentração simétrica)

\begin{tabular}{cc|ccc}
\hline & & Modelo 1 & Modelo 2 & Modelo 3 \\
\hline & $\mathrm{r}=15$ & 0.195 & 0.23 & 0.123 \\
$\mathrm{n}=10$ & $\mathrm{r}=25$ & 0.172 & 0.199 & 0.083 \\
& $\mathrm{r}=100$ & 0.181 & 0.196 & 0.064 \\
& $\mathrm{r}=150$ & 0.186 & 0.191 & 0.063 \\
& $\mathrm{r}=15$ & 0.165 & 0.217 & 0.094 \\
$\mathrm{n}=15$ & $\mathrm{r}=25$ & 0.16 & 0.182 & 0.082 \\
& $\mathrm{r}=100$ & 0.179 & 0.189 & 0.066 \\
& $\mathrm{r}=150$ & 0.178 & 0.185 & 0.055 \\
\hline & $\mathrm{r}=15$ & 0.175 & 0.226 & 0.105 \\
$\mathrm{n}=50$ & $\mathrm{r}=50$ & 0.168 & 0.178 & 0.059 \\
$\mathrm{r}=100$ & 0.18 & 0.192 & 0.065 \\
$\mathrm{r}=150$ & 0.193 & 0.198 & 0.061 \\
\hline
\end{tabular}

Notamos claramente que os Modelos 1 e 2 não são adequados, pois o nível de significância estimado não se aproxima do teórico nem para grandes amostras, no entanto o Modelo 3 se comporta adequadamente. A figura 3.14 mostra esse efeito graficamente. 
Figura 3.12: Gráfico do nível de significância estimado dos modelos 1, 2 e 3 (concentração simétrica)
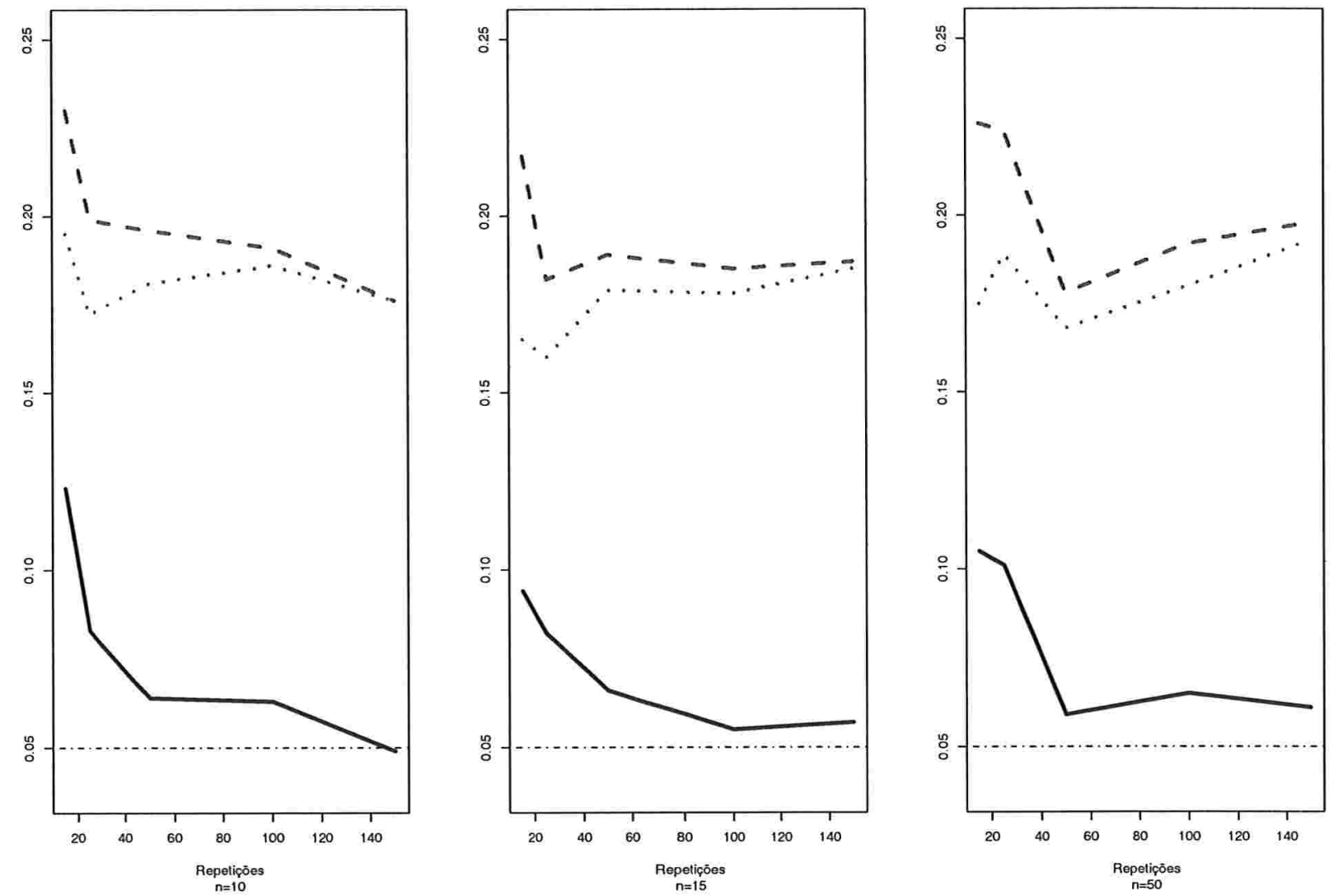


\section{concentração assimétrica - gama}

Consideramos os mesmos parâmetros da simulação passada (caso em que a concentração é simétrica). Aqui iremos adotar uma distribuição assimétrica para a concentração, a fim de verificar se o comportamento na estimação dos parâmetros e nos testes permanece o mesmo onde a concentração é simétrica. Abaixo estão os resultados das simulações de Monte Carlo considerando $x_{i} \sim$ Gama(escala $=$ 0.54 , forma $=4.76)$, ou seja, uma distribuição assimétrica para a verdadeira concentração,

Tabela 3.19: EQM e Viés estimados para os Modelos 1, 2 e 3 (concentração assimétrica)

\begin{tabular}{|c|c|c|c|c|c|c|c|c|}
\hline & & & \multicolumn{2}{|c|}{ Modelo 1} & \multicolumn{2}{|c|}{ Modelo 2} & \multicolumn{2}{|c|}{ Modelo 3} \\
\hline & & & $\alpha$ & $\beta$ & $\alpha$ & $\beta$ & $\alpha$ & $\beta$ \\
\hline \multirow{10}{*}{$\mathrm{n}=10$} & \multirow[t]{2}{*}{$\mathrm{r}=15$} & EQM & 0.05202 & 0.01187 & 0.05164 & 0.0118 & 0.05215 & 0.01195 \\
\hline & & Viés & -0.00486 & 0.00276 & -0.01417 & 0.00775 & -0.00305 & 0.00205 \\
\hline & \multirow[t]{2}{*}{$\mathrm{r}=25$} & EQM & 0.02788 & 0.00635 & 0.02803 & 0.00636 & 0.02774 & 0.00632 \\
\hline & & Viés & -0.00415 & 0.00181 & -0.0076 & 0.00347 & -0.00145 & 0.00025 \\
\hline & \multirow[t]{2}{*}{$r=50$} & EQM & 0.01527 & 0.00338 & 0.01524 & 0.00337 & 0.01497 & 0.00332 \\
\hline & & Viés & -0.00139 & 0.00188 & -0.0021 & 0.0022 & 0.00031 & 0.0009 \\
\hline & \multirow[t]{2}{*}{$r=100$} & EQM & 0.00722 & 0.00162 & 0.00722 & 0.00161 & 0.00726 & 0.00162 \\
\hline & & Viés & 0.00182 & -0.0003 & 0.00166 & -0.00021 & 0.00314 & -0.00104 \\
\hline & \multirow[t]{2}{*}{$r=150$} & EQM & 0.00509 & 0.0011 & 0.00509 & 0.0011 & 0.00505 & 0.0011 \\
\hline & & Viés & -0.00103 & 0.00042 & -0.00112 & 0.00046 & -0.00016 & $-4 e-05$ \\
\hline
\end{tabular}


Tabela 3.20: EQM e Viés estimados para os Modelos 1, 2 e 3 (concentração assimétrica)

\begin{tabular}{|c|c|c|c|c|c|c|c|c|}
\hline & & & \multicolumn{2}{|c|}{ Modelo 1} & \multicolumn{2}{|c|}{ Modelo 2} & \multicolumn{2}{|c|}{ Modelo 3} \\
\hline & & & $\alpha$ & $\beta$ & $\alpha$ & $\beta$ & $\alpha$ & $\beta$ \\
\hline \multirow{9}{*}{$\mathrm{n}=15$} & \multirow[t]{2}{*}{$\mathrm{r}=15$} & EQM & 0.02678 & 0.00709 & 0.02664 & 0.007 & 0.02677 & 0.00707 \\
\hline & & Viés & -0.00344 & 0.0032 & -0.01153 & 0.0076 & -0.00063 & 0.00182 \\
\hline & \multirow[t]{2}{*}{$\mathrm{r}=25$} & EQM & 0.01658 & 0.00398 & 0.01661 & 0.004 & 0.01631 & 0.00393 \\
\hline & & Viés & 0.00266 & -0.00094 & -0.00061 & 0.00072 & 0.00509 & -0.00219 \\
\hline & \multirow[t]{2}{*}{$\mathrm{r}=50$} & EQM & 0.00809 & 0.00189 & 0.00806 & 0.00188 & 0.00808 & 0.00188 \\
\hline & & Viés & 0.00228 & -0.00092 & 0.0017 & -0.00064 & 0.00401 & -0.00193 \\
\hline & \multirow[t]{2}{*}{$\mathrm{r}=100$} & EQM & 0.00356 & 0.00088 & 0.00356 & 0.00088 & 0.00354 & 0.00087 \\
\hline & & Viés & -0.00375 & 0.00203 & -0.00394 & 0.00215 & -0.00259 & 0.00138 \\
\hline & \multirow[t]{2}{*}{$r=150$} & EQM & 0.00256 & 0.00061 & 0.00256 & 0.00061 & 0.00256 & 0.00061 \\
\hline \multirow{11}{*}{$\mathrm{n}=50$} & & Viés & -0.00058 & 0.00022 & -0.00067 & 0.00027 & 0.00007 & -0.00015 \\
\hline & \multirow[t]{2}{*}{$\mathrm{r}=15$} & EQM & 0.00509 & 0.00142 & 0.00524 & 0.00147 & 0.00507 & 0.00141 \\
\hline & & Viés & -0.00325 & 0.00308 & -0.00937 & 0.00706 & -0.00105 & 0.00187 \\
\hline & \multirow[t]{2}{*}{$\mathrm{r}=25$} & EQM & 0.00329 & 0.00092 & 0.00328 & 0.00092 & 0.00327 & 0.00091 \\
\hline & & Viés & -0.00384 & 0.00201 & -0.00569 & 0.00318 & -0.00158 & 0.00065 \\
\hline & \multirow[t]{2}{*}{$\mathrm{r}=50$} & EQM & 0.00162 & 0.00045 & 0.00163 & 0.00045 & 0.0016 & 0.00044 \\
\hline & & Viés & -0.00156 & 0.00085 & -0.00213 & 0.00117 & -0.00016 & 0 \\
\hline & \multirow[t]{2}{*}{$r=100$} & EQM & 0.00078 & 0.00023 & 0.00078 & 0.00023 & 0.00077 & 0.00023 \\
\hline & & Viés & -0.00191 & 0.00115 & -0.00205 & 0.00123 & -0.00115 & 0.00069 \\
\hline & \multirow[t]{2}{*}{$\mathrm{r}=150$} & EQM & 0.00054 & 0.00015 & 0.00054 & 0.00015 & 0.00053 & 0.00015 \\
\hline & & Viés & -0.00051 & 0.00043 & -0.00056 & 0.00046 & 0.00009 & 0.00007 \\
\hline
\end{tabular}


$\mathrm{Na}$ maioria das combinações o Modelo 3 apresentou EQM menor, em alguns casos (nas combinações $n=10$ e $r=15 ; n=15$ e $r=15 ; n=15$ e $r=50$ ) o Modelo 2 apresentou menor EQM. Apesar das diferenças os resultados são muito próximos.

Figura 3.13: Gráfico do viés dos parâmetros dos Modelos 1, 2 e 3 (concentração assimétrica)
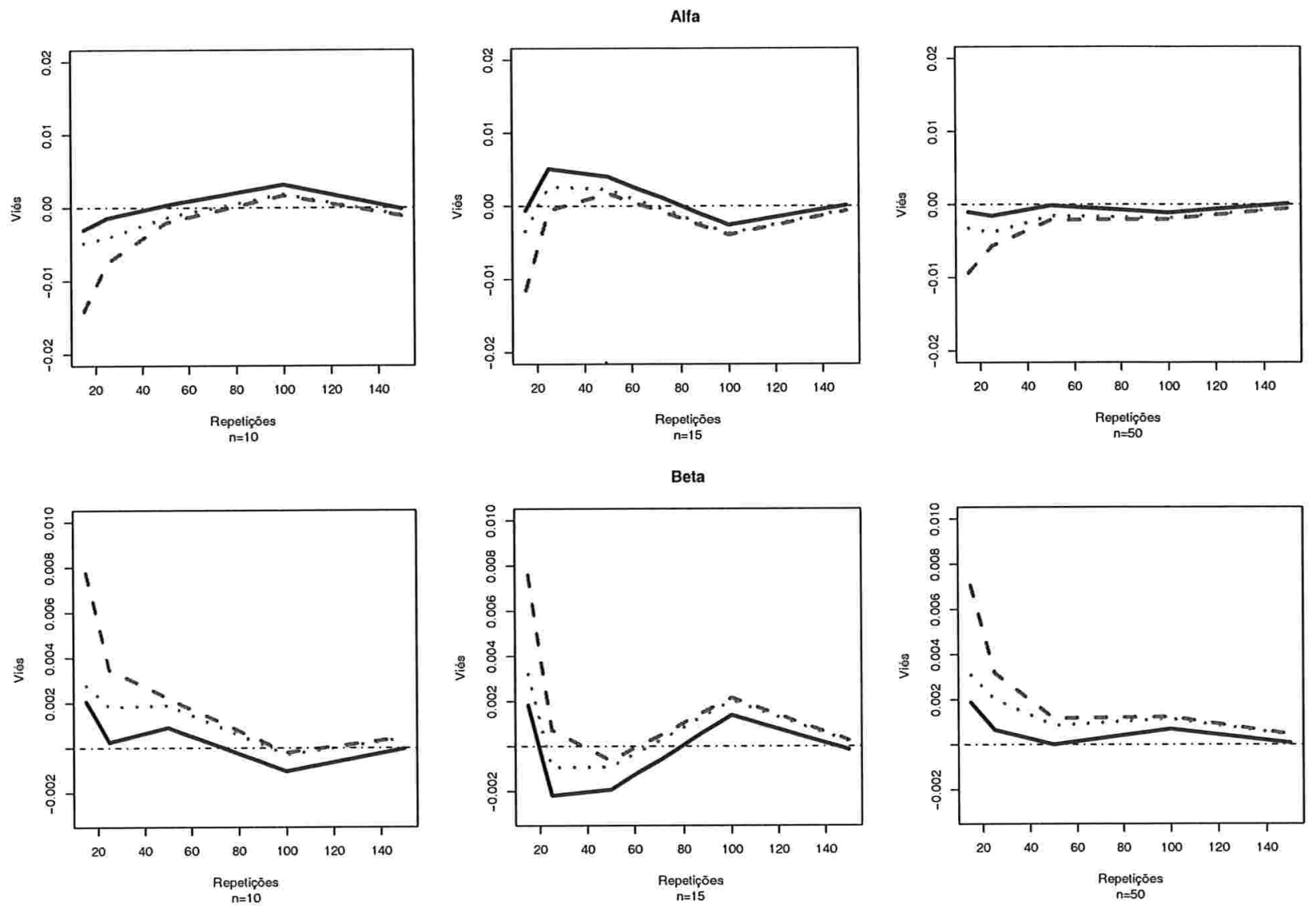

O comportamento dos vieses dos parâmetros para três Modelos propostos não diferem muito entre si, apenas para $n=50$ o Modelo 3 tem menos viés que os demais para todas as repetições. A tabela abaixo nos mostra o nível de significância estimado para os três modelos quando a concentração é assimétrica. 
Tabela 3.21: Nível de significância estimado para os Modelos 1, 2 e 3 (concentração assimétrica)

\begin{tabular}{cc|ccc}
\hline & & Modelo 1 & Modelo 2 & Modelo 3 \\
\hline & $\mathrm{r}=15$ & 0.199 & 0.231 & 0.117 \\
$\mathrm{n}=10$ & $\mathrm{r}=50$ & 0.177 & 0.196 & 0.07 \\
& $\mathrm{r}=100$ & 0.182 & 0.195 & 0.067 \\
& $\mathrm{r}=150$ & 0.181 & 0.186 & 0.06 \\
\hline & $\mathrm{r}=15$ & 0.179 & 0.212 & 0.125 \\
$\mathrm{n}=15$ & $\mathrm{r}=50$ & 0.194 & 0.211 & 0.085 \\
& $\mathrm{r}=100$ & 0.183 & 0.193 & 0.07 \\
& $\mathrm{r}=150$ & 0.157 & 0.166 & 0.046 \\
& $\mathrm{r}=15$ & 0.17 & 0.223 & 0.105 \\
& $\mathrm{r}=25$ & 0.193 & 0.219 & 0.087 \\
$\mathrm{n}=50$ & $\mathrm{r}=50$ & 0.18 & 0.194 & 0.066 \\
$\mathrm{r}=100$ & 0.172 & 0.178 & 0.052 \\
$\mathrm{r}=150$ & 0.177 & 0.181 & 0.056 \\
\hline
\end{tabular}

A Tabela 3.21 indica que para as concentrações assimétricas o nível de significância estimado se compara ao da Tabela 3.18, ou seja, os Modelos 1 e 2 não são adequados nem para grandes amostras, já o Modelo 3 é adequado (para grandes amostras), pois seu nível de significância estimado converge para o teórico. 
Figura 3.14: Gráfico do nível de significância estimado dos modelos 1, 2 e 3 (concentração assimétrica)
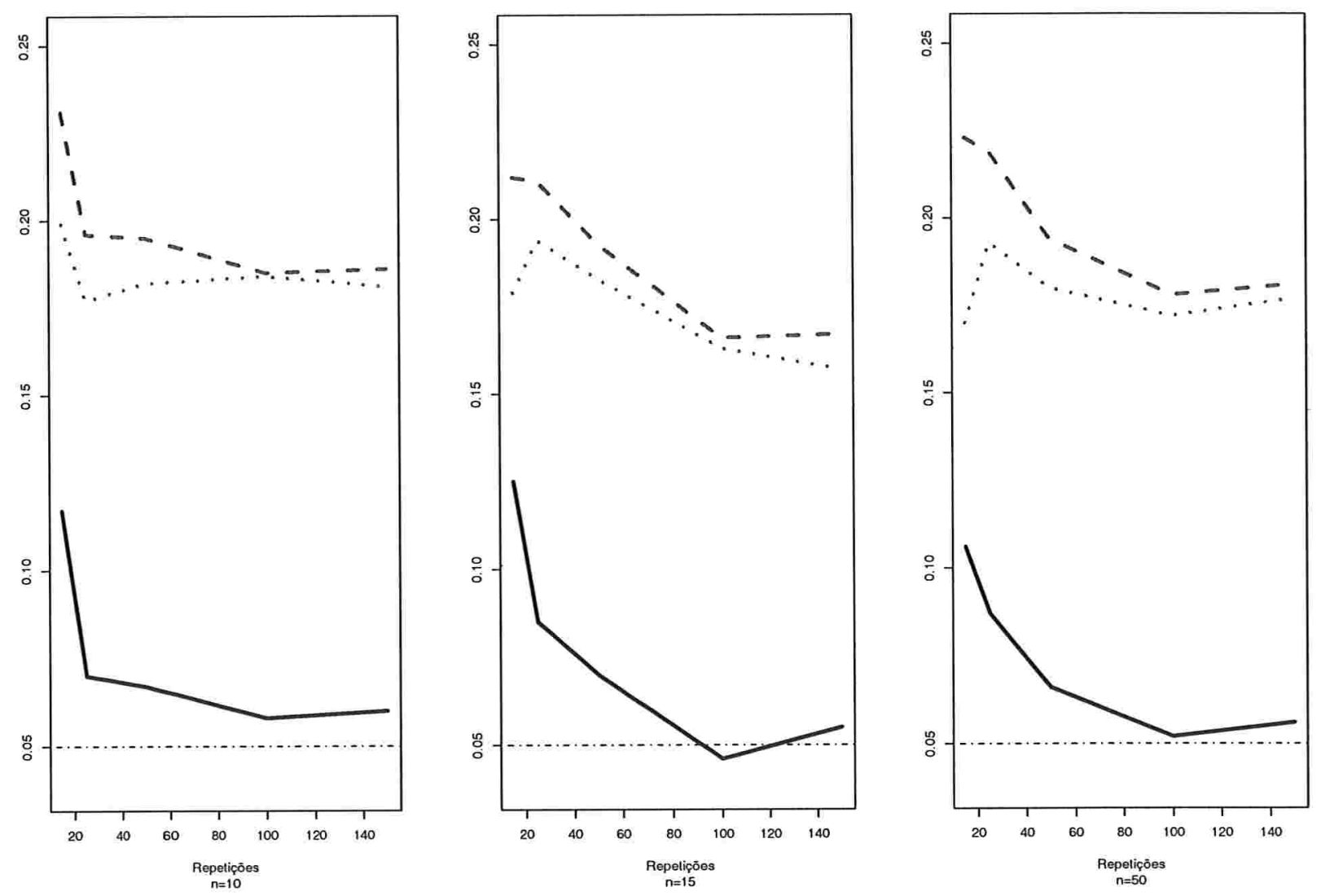

Nas seções acima concluímos que os Modelos 1 e 2 não são adequados quando uma estrutura autoregressiva de ordem 1 é imposta nas repetições de cada concentração. Para obter testes mais confiáveis, quando o número de repetições é pequeno, seria interessante corrigir, com alguma metodologia apropriada, os testes propostos para testar a tendência do método novo no no modelo que comporta a correlação entre as réplicas. 


\section{Capítulo 4}

\section{Aplicação}

Neste capítulo analisamos um conjunto de dados reais da companhia Vale do Rio Doce. Comparamos apenas dois instrumentos de medição. Utilizamos as duas abordagens estudadas neste trabalho: ambas medem a concentração com repetições, mas a diferença essencial entre as duas abordagens é que uma usa as repetições apenas para obter os valores das variâncias, após isso o modelo apresentado em (2.3) é utilizado para analisar o vetor observado $\left(\bar{Y}_{1}, \bar{X}_{1}\right), \ldots,\left(\bar{Y}_{n}, \bar{X}_{n}\right)$. A outra abordagem, apresentada em (2.5), trata todo o processo inferencial levando em conta as medidas repetidas da amostra.

\subsection{Análise de cobalto em jazidas}

O Cobalto é um elemento químico que na temperatura ambiente se encontra no estado sólido. Ele é utilizado para a produção de superligas usadas em turbinas de gás de aviões, ligas resistentes a corrosão e ferramentas de diamante. O metal não é encontrado em estado nativo, mas em diversos minerais, razão pela qual é extraído normalmente junto com outros produtos, especialmente como subproduto do níquel e do cobre. Utilizamos os dados coletados pela companhia Vale do Rio Doce do teor 
de cobalto em amostras retiradas de jazidas e avaliadas em 8 concentrações diferentes e 20 repetições para cada concentração. Três métodos de medição foram utilizados, a saber

GQ6 : Abertura ácida por água régia em $6 \mathrm{~g}$ de amostra, com leitura por espectrometria de plasma;

PLAA: Abertura total por fusão alcalina, com leitura por espectrometria de plasma;

RX : As amostras foram preparadas por pulverização, prensagem e leitura por espectrometria de raios X fluorescentes dispersivos em comprimento de onda.

Dentre os três métodos escolhemos GQ6 e PLAA, pois ambos apresentam características de uma distribuição normal. O método RX foi descartado por apresentar muitos valores discrepantes. Foi necessário retirar algumas unidades experimentais (concentrações) do conjunto de dados para que pudéssemos utilizar as metodologias apresentadas neste trabalho. Possivelmente as u.e. retiradas foram influenciadas por variáveis externas, pois os dados estavam com muitos pontos discrepantes. O método de referência (padrão) é o PLAA, portanto desejamos verificar se existe viés na medida da concentração de cobalto quando usamos o método GQ6, para isso propusemos quatro modelos funcionais heterocedásticos com erro nas variáveis. Aplicamos o logaritmo natural nas observações, pois algumas concentrações tinham o valor muito alto e variabilidade muito alta, o que ocasionava a instabilidade dos algoritmos.

Modelo 1 - Estimação das variâncias pelo método do momentos.

Este modelo trata as repetições apenas como auxílio para obter os valores das variâncias. O modelo é dado abaixo 


$$
\begin{aligned}
\bar{Y}_{i} & =\alpha+\beta x_{i}+e_{i} \\
\bar{X}_{i} & =x_{i}+u_{i}
\end{aligned}
$$

para $i=1,2, \ldots, 8 \mathrm{e}$

$\bar{Y}_{i}$ : Valor médio observado do logaritmo da $i$-ésima concentração de cobalto medida pelo método GQ6;

$\bar{X}_{i}$ : Valor médio observado do logaritmo da $i$-ésima concentração de cobalto medida pelo método PLAA;

$x_{i}$ : Logaritmo da $i$-ésima concentração de cobalto (não observada);

$\alpha$ : Viés aditivo do método GQ6;

$\beta$ : Viés multiplicativo do método GQ6;

$e_{i} \sim N\left(0, \lambda_{i}\right)$ e $u_{i} \sim N\left(0, \kappa_{i}\right)$. Os valores para $\lambda_{i}$ e $\kappa_{i}$ são obtidos da seguinte forma:

$$
\lambda_{i}=(1 / 20) \sum_{j=1}^{20} \frac{\left(Y_{i j}-\bar{Y}_{i}\right)^{2}}{19} \text { e } \kappa_{i}=(1 / 20) \sum_{j=1}^{20} \frac{\left(X_{i j}-\bar{X}_{i}\right)^{2}}{19}
$$

Modelo 2 - Estima as variâncias usando o método da máxima verossimilhança, supondo que as réplicas são não correlacionadas.

$$
\begin{aligned}
Y_{i j} & =\alpha+\beta x_{i}+e_{i j} \\
X_{i j} & =x_{i}+u_{i j}
\end{aligned}
$$

para $i=1,2, \ldots, 8 ; j=1,2, \ldots, 20 \mathrm{e}$

$Y_{i j}$ : Valor observado do logaritmo da $j$-ésima repetição na $i$-ésima concentração de cobalto medida pelo método GQ6;

$X_{i j}$ : Valor observado do logaritmo da $j$-ésima repetição na $i$-ésima concentração de cobalto medida pelo método PLAA;

$x_{i}$ : Logaritmo da $i$-ésima concentração de cobalto (não observada); 
$\alpha$ : Viés aditivo do método GQ6;

$\beta$ : Viés multiplicativo do método GQ6;

$e_{i j} \sim N\left(0, \lambda_{i}\right)$ e $u_{i j} \sim N\left(0, \kappa_{i}\right)$.

Modelo 3 - Estima as variâncias usando o método da máxima verossimilhança, supondo que as réplicas são correlacionadas. A estrutura de correlação considerada para as repetições é a $\mathrm{AR}(1)$ autoregressiva de ordem 1.

$$
\begin{aligned}
& \boldsymbol{Y}_{i}=\left(\alpha+\beta x_{i}\right) 1+\boldsymbol{e}_{i} \\
& \boldsymbol{X}_{i}=x_{i} \mathbf{1}+\boldsymbol{u}_{i}
\end{aligned}
$$

para $i=1,2, \ldots, 8 \mathrm{e}$

$\boldsymbol{Y}_{i}$ : Vetor observado do logaritmo da $i$-ésima concentração de cobalto medida pelo método GQ6;

$\boldsymbol{X}_{i}$ : Vetor observado do logaritmo da $i$-ésima concentração de cobalto medida pelo método PLAA;

$x_{i}$ : Logaritmo da $i$-ésima concentração de cobalto (não observada);

$\alpha$ : Viés aditivo do método GQ6;

$\beta$ : Viés multiplicativo do método GQ6;

$e_{i} \sim N_{20}\left(0, \lambda_{i} \Omega\left(\rho_{1}\right)\right)$ e $u_{i} \sim N_{20}\left(0, \kappa_{i} \Omega\left(\rho_{2}\right)\right)$ com

$$
\Omega\left(\rho_{k}\right)=\left[\begin{array}{ccccc}
1 & \rho_{k} & \rho_{k}^{2} & \ldots & \rho_{k}^{19} \\
\rho_{k} & 1 & \rho_{k} & \ldots & \rho_{k}^{18} \\
\rho_{k}^{2} & \rho_{k} & 1 & \ldots & \rho_{k}^{17} \\
\vdots & \vdots & \vdots & \ddots & \vdots \\
\rho_{k}^{19} & \rho_{k}^{18} & \rho^{17} & \ldots & 1
\end{array}\right] \text {, para } k=1,2
$$

Modelo 4 - Estima as variâncias usando o método da máxima verossimilhança, supondo que as réplicas são correlacionadas. A estrutura de correlação considerada 
para as repetições é a MA(1) média móvel de ordem 1.

O Modelo 4 é idêntico ao Modelo 3 a menos da matriz de correlação, neste Modelo a matriz de correlação é dada por

$$
\Omega\left(\rho_{k}\right)=\left[\begin{array}{ccccc}
1 & \rho_{k} & 0 & \ldots & 0 \\
\rho_{k} & 1 & \rho_{k} & \ldots & 0 \\
0 & \rho_{k} & 1 & \ldots & 0 \\
\vdots & \vdots & \vdots & \ddots & \vdots \\
0 & 0 & 0 & \ldots & 1
\end{array}\right], \text { para } k=1,2
$$

\subsection{Análise descritiva}

Faremos uma breve análise descritiva, para identificar o comportamento das concentrações mensuradas. Na tabela abaixo mostramos as médias de cada log-concentração medida pelos métodos PLAA e GQ6.

Tabela 4.1: Médias em cada log-concentração

\begin{tabular}{rrrrrrrrr}
\hline \multicolumn{8}{c}{$\log$ das Concentrações } \\
& $\log x_{1}$ & $\log x_{2}$ & $\log x_{3}$ & $\log x_{4}$ & $\log x_{5}$ & $\log x_{6}$ & $\log x_{7}$ & $\log x_{8}$ \\
\hline PLAA & 4.14 & 4.58 & 8.82 & 4.30 & 4.15 & 4.39 & 4.28 & 4.94 \\
GQ6 & 3.53 & 4.64 & 8.86 & 3.69 & 3.34 & 3.88 & 3.15 & 4.18 \\
\hline
\end{tabular}

Percebe-se na Tabela (4.1) que, aparentemente, existe diferenças entre as medidas dos métodos. Na maioria das concentrações o método GQ6 parece subestimar a verdadeira log-concentração. Na tabela abaixo mostramos as variâncias de cada log-concentração medida pelos métodos PLAA e GQ6. 
Tabela 4.2: Variâncias em cada log-concentração

\begin{tabular}{rrrrrrrrr}
\hline & & \multicolumn{8}{c}{$\log$ das concentrações } \\
& $\log x_{1}$ & $\log x_{2}$ & $\log x_{3}$ & $\log x_{4}$ & $\log x_{5}$ & $\log x_{6}$ & $\log x_{7}$ & $\log x_{8}$ \\
\hline PLAA & 0.0017 & 0.0012 & 0.0001 & 0.0018 & 0.0048 & 0.0083 & 0.0076 & 0.0038 \\
GQ6 & 0.0010 & 0.0030 & 0.0006 & 0.0005 & 0.0009 & 0.0005 & 0.0006 & 0.0003 \\
\hline
\end{tabular}

Nota-se que as variâncias dependem da concentração mensurada, caracterizando dados heterocedásticos. Na maioria das concentrações a variabilidade do método GQ6 é menor que a de PLAA.

Figura 4.1: Boxplot para cada concentração medida por PLAA
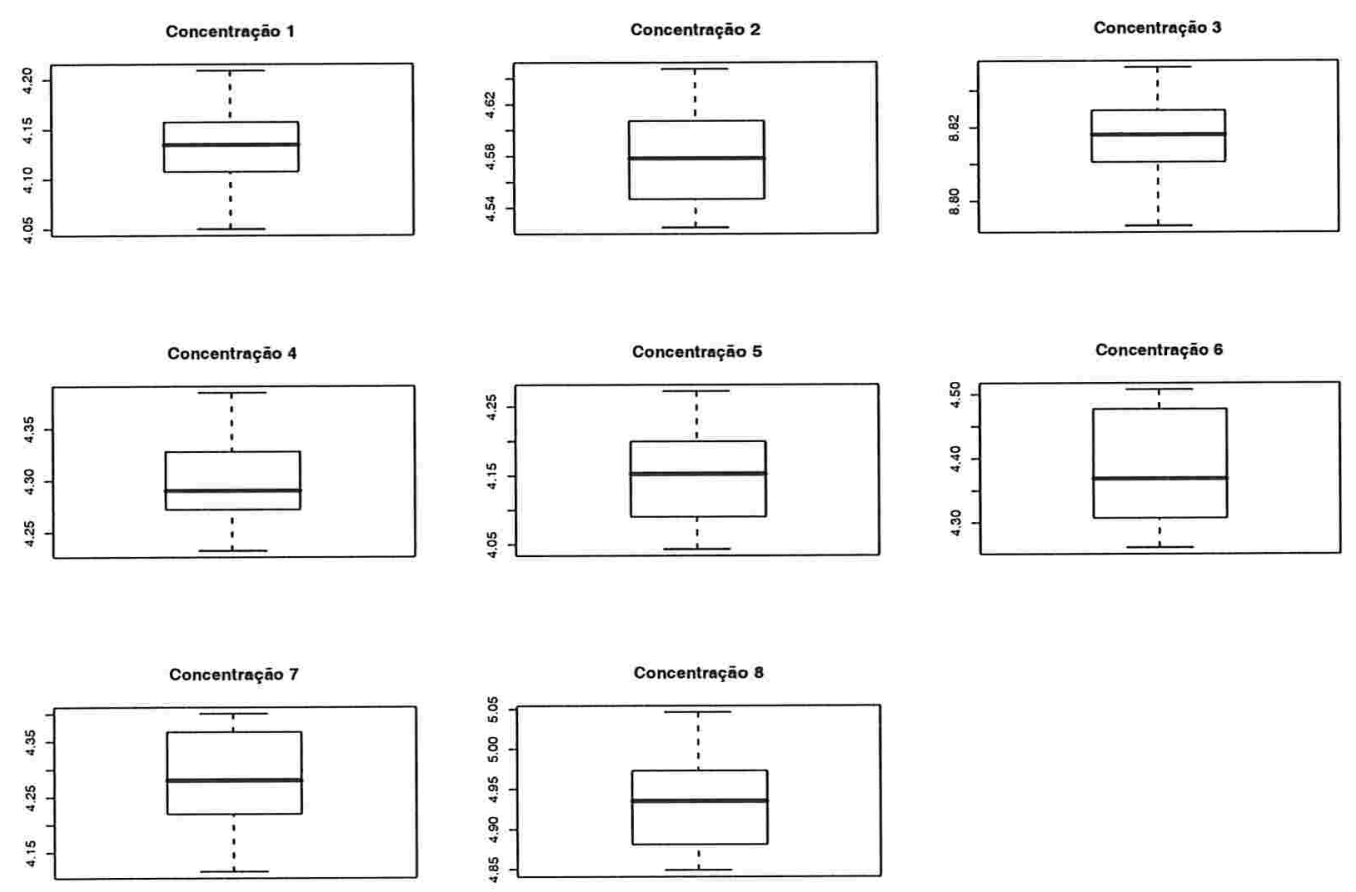
Figura 4.2: Boxplot para cada concentração medida por GQ6
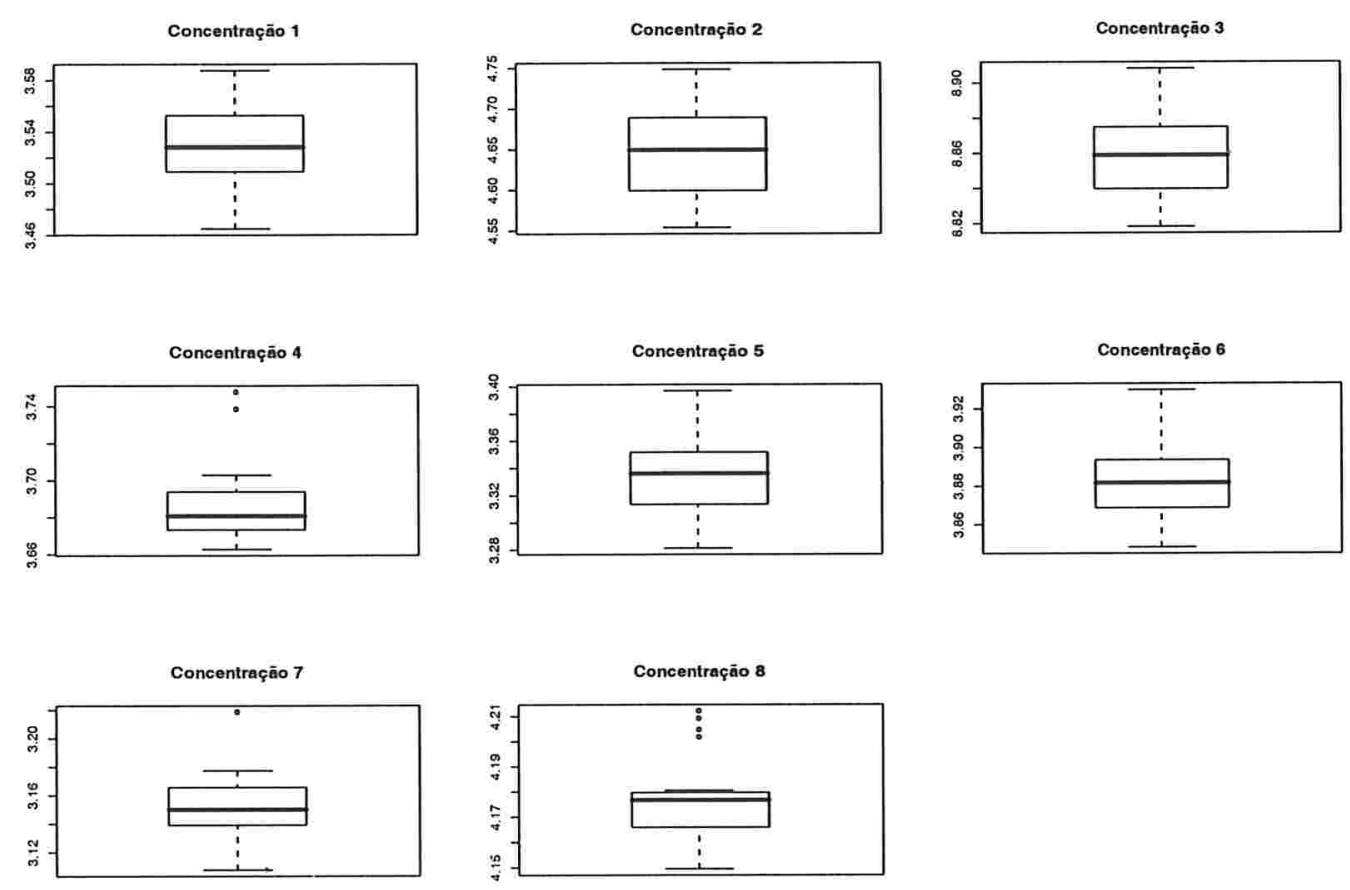

As Figuras 4.1 e 4.2 mostram os boxplot para os métodos em questão, percebemos que as observações são simétricas para cada concentração, mas para as concentrações 4 e 8 do método GQ6 observamos alguns pontos discrepantes. Abaixo mostramos os gráficos de autocorrelação e autocorrelação parcial das observações para cada método. 
Figura 4.3: Gráficos de Autocorrelações para PLAA
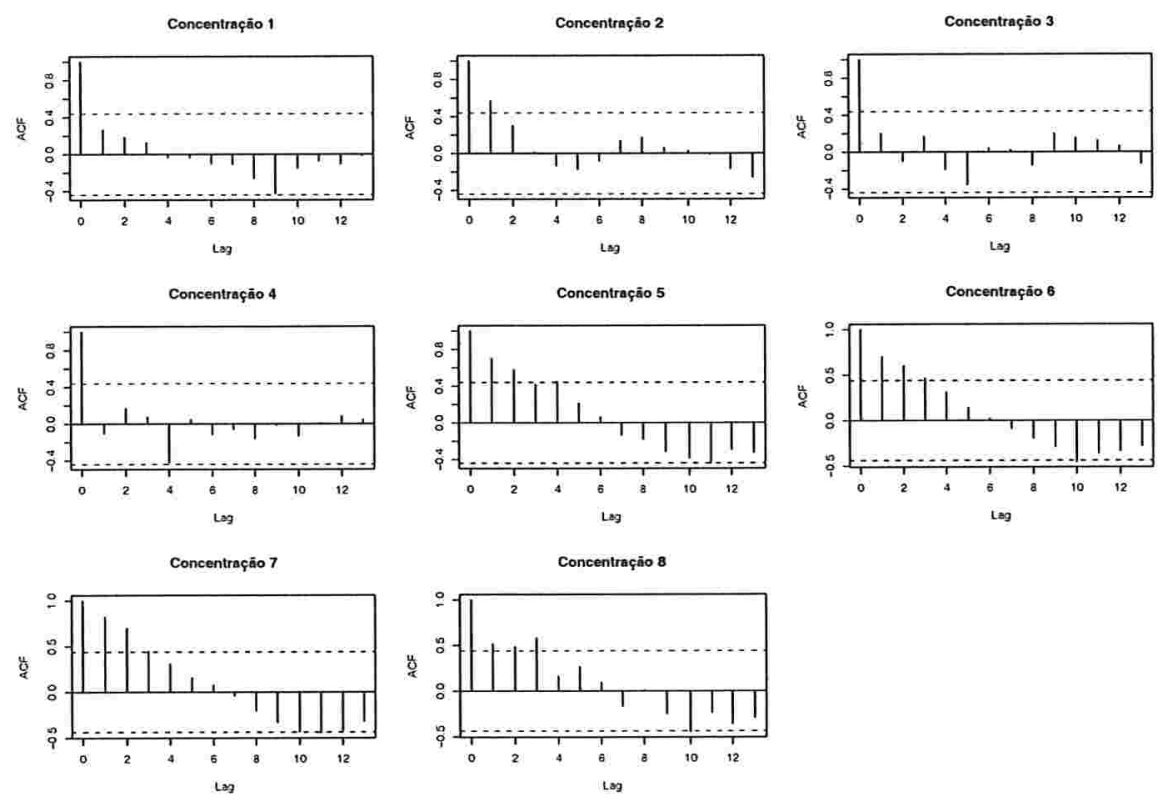

Figura 4.4: Gráficos de Autocorrelações parciais para PLAA
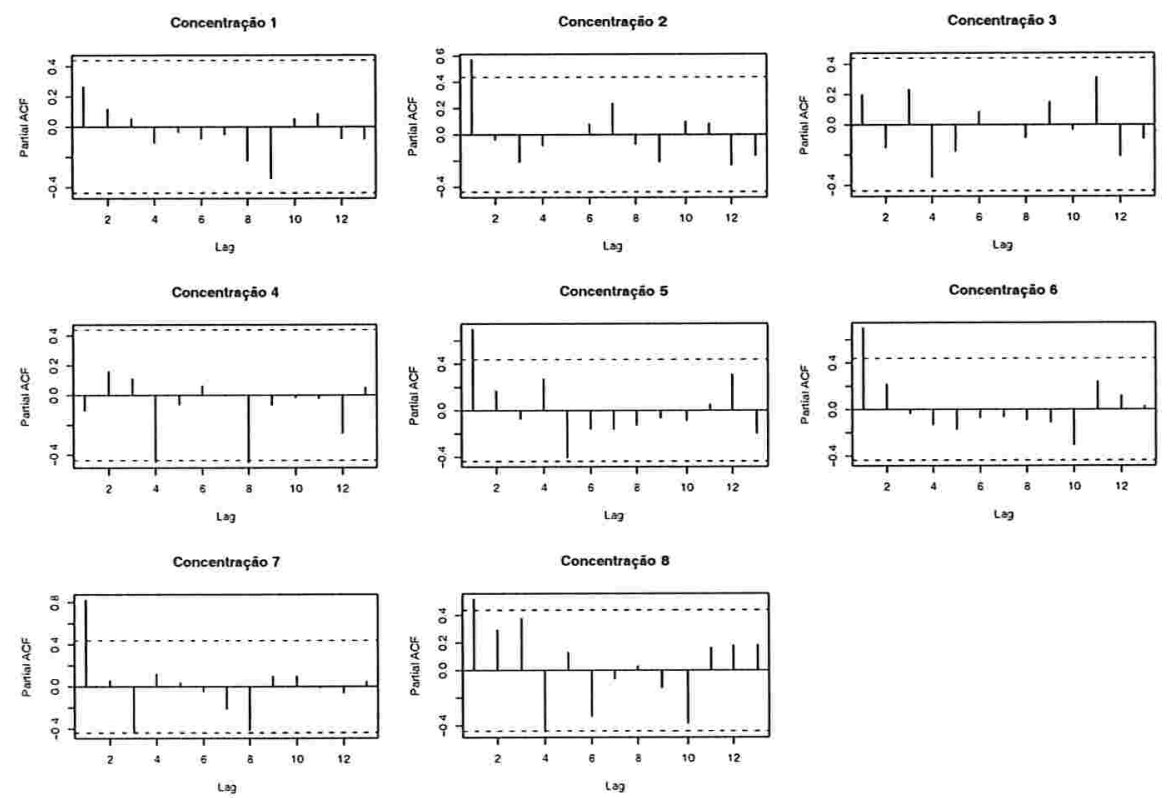
Figura 4.5: Gráficos de Autocorrelações para GQ6
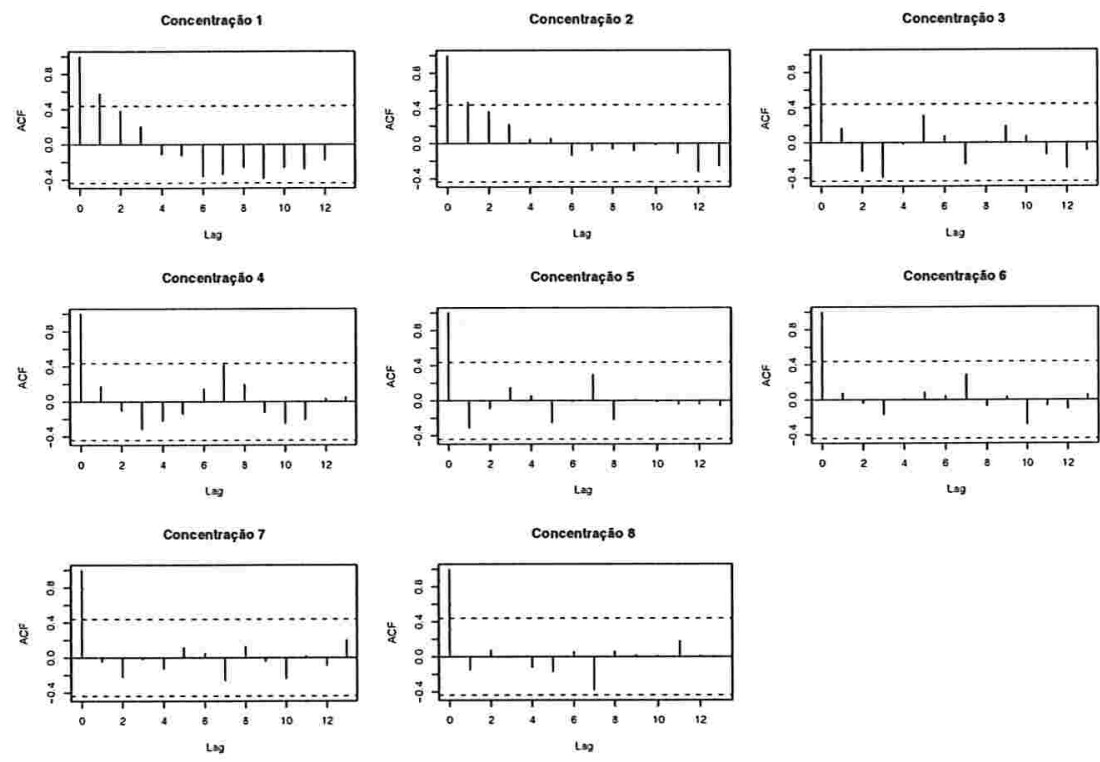

Figura 4.6: Gráficos de Autocorrelações parciais para GQ6
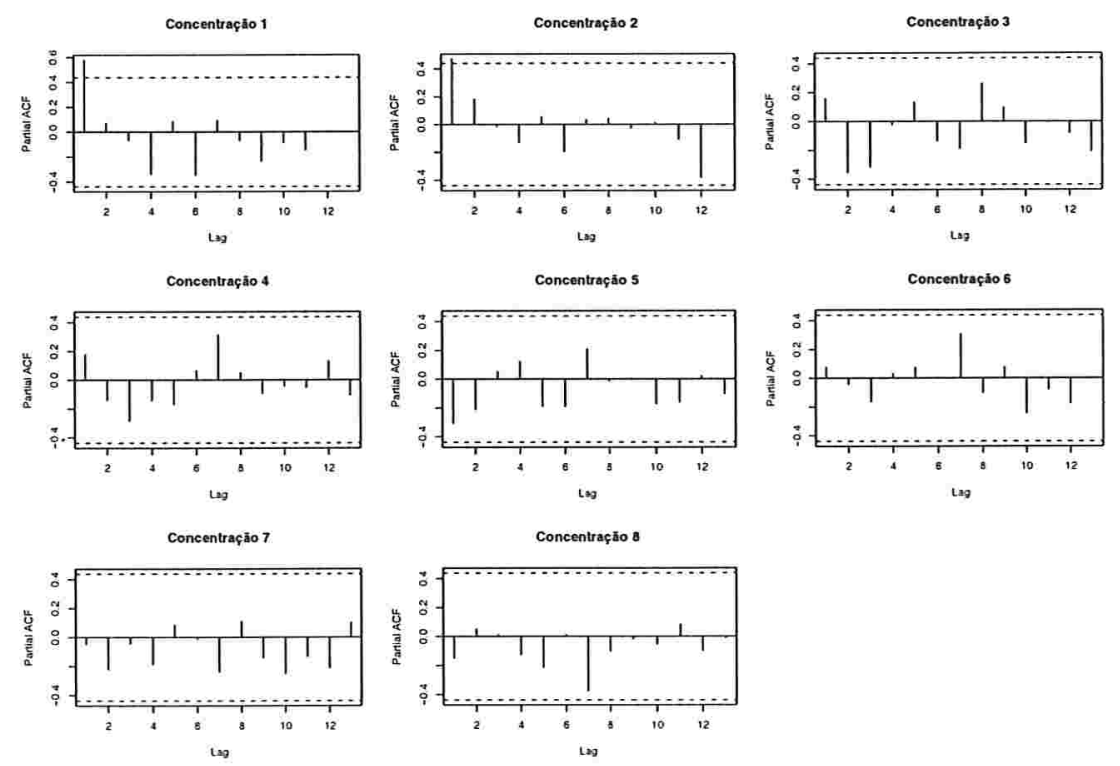
Notamos nas Figuras 4.3-4.6 que existe alguma correlação entre as observações de algumas concentrações, principalmente no método padrão PLAA. Percebemos que nas concentrações $1,2,4,5,6,7$ e 8 existe alguma evidência de autocorrelação (e autocorrelação parcial, a menos na primeira concentração) entre as repetições no método PLAA, já no método GQ6 as concentrações 1, 2 e 4 apresentaram autocorrelação significativa e 1 e 2 autorrelação parcial significativa. Abaixo mostramos o gráfico das observações.

Figura 4.7: Gráficos das observações
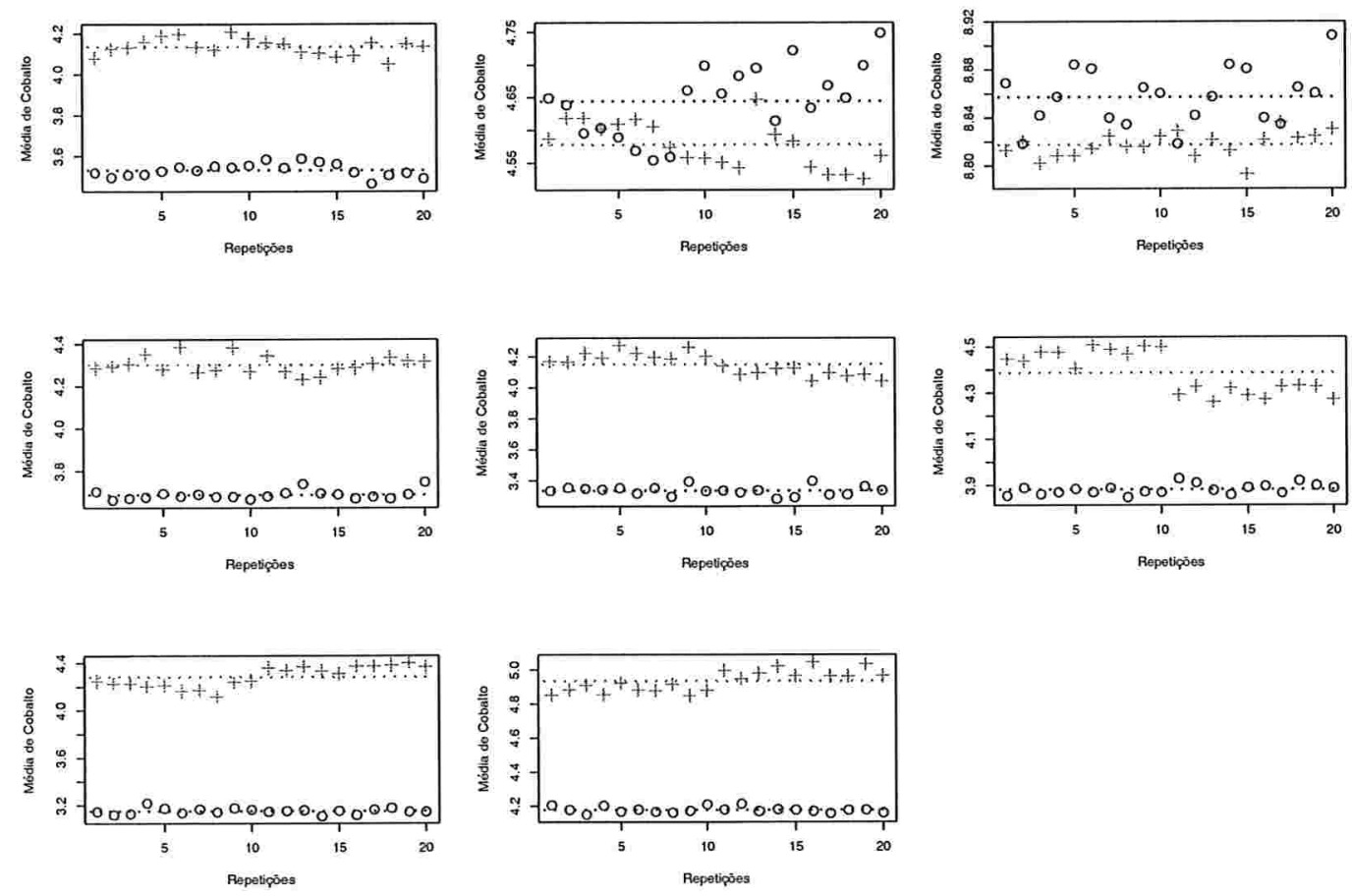

Na figura 4.8, consideramos bola para as observações mensuradas usando o GQ6 e cruz para as observações mensuradas usando o PLAA. Notamos, como visto na Tabela (4.1) a diferença entre os métodos. 
Figura 4.8: Gráfico das Médias

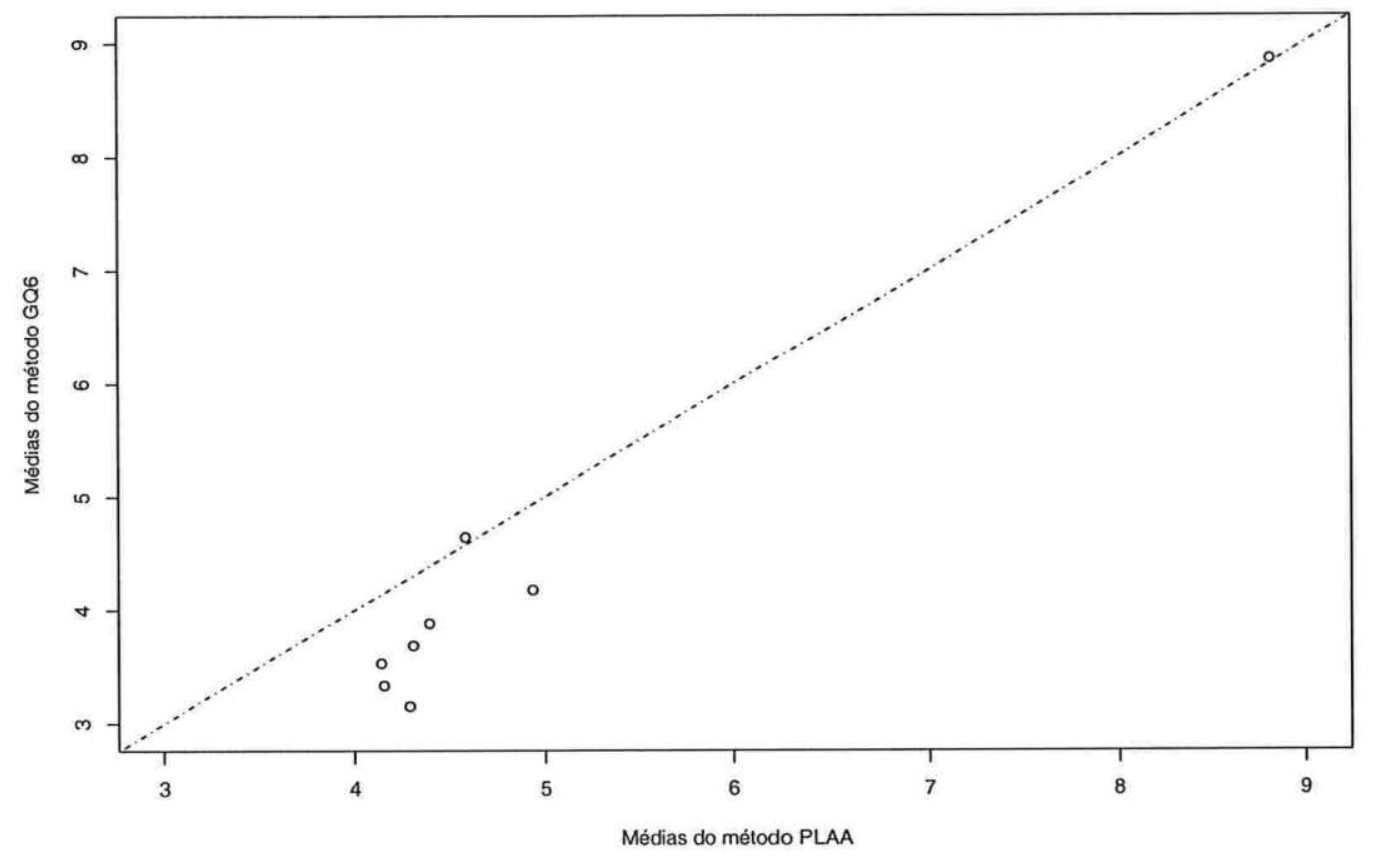

O gráfico acima, obtido plotando as médias de cada concentração, nos dá uma idéia do viés do método GQ6. A linha pontilhada indica a situação ideal, ou seja, a situação em que não existe viés.

\subsection{Análise inferencial}

Nesta seção faremos uma análise inferencial usando os quatro modelos propostos. Abaixo estão as estimativas para os parâmetros dos modelos. 
Tabela 4.3: Estimativas (e erro-padrão) dos vieses para cada modelo

\begin{tabular}{r|r|r|r|r}
\hline & $\begin{array}{r}\text { Modelo 1 } \\
\text { s/ réplicas }\end{array}$ & $\begin{array}{r}\text { Modelo 2 } \\
\text { réplicas ind. }\end{array}$ & $\begin{array}{r}\text { Modelo 3 } \\
\text { AR(1) }\end{array}$ & $\begin{array}{r}\text { Modelo 4 } \\
\text { MA(1) }\end{array}$ \\
\hline \hline$\alpha$ & $-1.22(0.0131)$ & $-1.22(0.0172)$ & $-1.34(0.2506)$ & $-1.25(0.0272)$ \\
$\beta$ & $1.14(0.0019)$ & $1.14(0.0023)$ & $1.16(0.0469)$ & $1.15(0.0034)$ \\
$\rho_{1}$ & - & - & $0.99(0.423)$ & $0.16(0.0736)$ \\
$\rho_{2}$ & - & - & $0.60(0.087)$ & $0.46(0.0176)$ \\
Wald & 9905.2 & 5380.8 & 61.6 & 2140.2 \\
Valor-P & $<0.001$ & $<0.001$ & $<0.001$ & $<0.001$ \\
AIC & - & -79.2 & -555.4 & -115.2 \\
Log-Lik & - & 77.6 & 317.7 & 97.6 \\
\hline
\end{tabular}

Concluímos que o método GQ6 mensura a concentração com viés, tanto aditivo $(\alpha)$ quanto multiplicativo $(\beta)$. A análise descritiva já apontava esse resultado. Os 4 modelos retornam resultados bem próximos para as estimativas dos vieses, porém verificamos que as repetições são correlacionadas, pois os parâmetros autoregressivo (Modelo 3) e de média móvel (Modelo 4) são significativos. Com os resultados das simulações feitas na seção passada verificamos que os modelos 1 e 2 não são adequados para tratar dados com repetições correlacionadas, por isso aconselhamos usar um modelo que leve em conta a correlação entre as réplicas. O Modelo 3 é escolhido como mais adequado dentre os modelos apresentados, pois é o que possui maior Log-lik e menor AIC.

Poderíamos formular outro modelo em que a estrutura de correlação seja flexível para cada concentração, assim, seria possível admitir que as repetições em algumas concentrações são independentes ou estruturas de correlação diferentes para as repetições de cada concentração, bastaria considerar $\Omega_{i}($.$) para i=1,2, \ldots, n$. A tabela abaixo nos retorna as estimativas para as variâncias e log das concentrações. 
Tabela 4.4: Estimativas das variâncias e concentrações para cada modelo

\begin{tabular}{lllll}
\hline & Modelo 1 & Modelo 2 & Modelo 3 & Modelo 4 \\
\hline$\lambda_{1}$ & 0.0010 & 0.0012 & 0.0384 & 0.0009 \\
$\lambda_{1}$ & 0.0030 & 0.4716 & 0.1611 & 0.0026 \\
$\lambda_{1}$ & 0.0006 & 0.0006 & 0.0398 & 0.0005 \\
$\lambda_{1}$ & 0.0005 & 0.0005 & 0.0294 & 0.0004 \\
$\lambda_{1}$ & 0.0009 & 0.0010 & 0.1206 & 0.0010 \\
$\lambda_{1}$ & 0.0005 & 0.0005 & 0.0420 & 0.0005 \\
$\lambda_{1}$ & 0.0006 & 0.0007 & 0.0776 & 0.0006 \\
$\lambda_{1}$ & 0.0003 & 0.0003 & 0.0341 & 0.0003 \\
\hline$\kappa_{1}$ & 0.0017 & 0.0019 & 0.0015 & 0.0034 \\
$\kappa_{1}$ & 0.0012 & 0.0013 & 0.0007 & 0.1699 \\
$\kappa_{1}$ & 0.0001 & 0.0001 & 0.0001 & 0.0001 \\
$\kappa_{1}$ & 0.0018 & 0.0018 & 0.0023 & 0.0046 \\
$\kappa_{1}$ & 0.0048 & 0.0316 & 0.0020 & 0.0164 \\
$\kappa_{1}$ & 0.0083 & 0.0145 & 0.0035 & 0.0125 \\
$\kappa_{1}$ & 0.0076 & 0.2264 & 0.0024 & 0.1130 \\
$\kappa_{1}$ & 0.0038 & 0.0509 & 0.0024 & 0.0254 \\
\hline $\log \left(x_{1}\right)$ & 4.1459 & 4.1505 & 4.1325 & 4.1648 \\
$\log \left(x_{2}\right)$ & 4.7694 & 4.5796 & 4.5784 & 5.1368 \\
$\log \left(x_{3}\right)$ & 8.8155 & 8.8174 & 8.8180 & 8.8172 \\
$\log \left(x_{4}\right)$ & 4.2900 & 4.2960 & 4.3040 & 4.3064 \\
$\log \left(x_{5}\right)$ & 4.0010 & 3.9902 & 4.1435 & 4.0033 \\
$\log \left(x_{6}\right)$ & 4.4552 & 4.4632 & 4.3876 & 4.4744 \\
$\log \left(x_{7}\right)$ & 3.8465 & 3.8271 & 4.2809 & 3.8400 \\
$\log \left(x_{8}\right)$ & 4.7281 & 4.7240 & 4.9274 & 4.7347 \\
\hline & & & & \\
\hline
\end{tabular}




\section{Capítulo 5}

\section{Conclusões e comentários finais}

\subsection{Conclusões}

Neste trabalho propomos alguns modelos para tratar uma relação linear simples onde a verdadeira covariável não é observada e repetições são feitas em cada inidades experimental. Consideramos repetições correlacionadas ou não. Uma aplicação destes modelos é a validação de métodos de medição. Concluímos, via simulação, que o modelo que incorpora as repetições no processo de estimação (2.15) é mais adequado que o modelo usual (2.7). Esse resultado só é válido para os parâmetros fixados e se as suposições estiverem satisfeitas. Vimos também na Tabela 3.18 que quando as réplicas são dependentes o modelo usual e o modelo (2.15) não são adequados. Portanto é importante fazer uma análise descritiva e, de preferência, usar um modelo que considere repetições correlacionadas para testar se a correlação é significativa.

Disponibilizamos os algoritmos utilizados neste trabalho no seguinte endereço http://www.ime.usp.br/ patriota/Dissertacao/Algoritmo.R

\subsection{Pesquisas futuras}

Existem diversas extensões que podem ser feitas, a saber

- Modelar uma possível correlação entre os métodos de mensuração; 
- Flexibilizar a distribuição dos erros para comportar dados discrepantes;

- Considerar uma distribuição assimétrica para a verdadeira concentração;

- Flexibilizar a estrutura de correlação para as repetições de cada concentração;

- Verificar como se comporta o modelo se a estrutura de correlação for mal especificada e/ou distribuição para os erros diferente da gaussiana;

- Fazer um estudo de simulação dos parâmetros que modelam a covariância das repetições $\left(\rho_{1}\right.$ e $\left.\rho_{2}\right)$ para algumas estruturas $\Omega($.$) conhecidas e analisar o nível$ de significância empírico do teste $\Omega\left(\rho_{1}\right)=\Omega\left(\rho_{2}\right)=\boldsymbol{I}$.

- Estender os resultados obtidos neste trabalho para dados desbalanceados. 


\section{Apêndice A}

\section{Resultados}

\section{A.1 Dois métodos}

Considerando os vetores aleatórios $\left(\boldsymbol{Y}_{1}, \boldsymbol{X}_{1}\right), \ldots,\left(\boldsymbol{Y}_{n}, \boldsymbol{X}_{n}\right)$ com distribuição dada em (2.21), demonstraremos alguns resultados importantes que foram utilizados neste trabalho. Assim,

$$
Z_{i}=\left(\begin{array}{c}
Y_{i} \\
X_{i}
\end{array}\right) \sim N_{2 m}\left[\left(\begin{array}{c}
\left(\alpha 1+\beta \boldsymbol{a}_{i} x_{i}\right) \\
\boldsymbol{b}_{i} x_{i}
\end{array}\right) ;\left(\begin{array}{cc}
\lambda_{i} \Omega_{i}\left(\rho_{1}\right) & 0 \\
0^{\top} & \kappa_{i} \Omega_{i}\left(\rho_{2}\right)
\end{array}\right)\right] \forall i \in I
$$

com $\boldsymbol{a}_{i}=\left(a_{i 1}, a_{i 2}, \ldots, a_{i m}\right)^{\top}$ e $\boldsymbol{b}_{i}=\left(b_{i 1}, b_{i 2}, \ldots, b_{i m}\right)^{\top}$. O log da função de verossimilhança é dada abaixo

$$
\ell(\theta)=\sum_{i \in I} \ell_{i}(\theta)
$$

sendo

$$
\ell_{i}(\boldsymbol{\theta})=-m \log (2 \pi)-\frac{m}{2} \log \left(\kappa_{i}\right)-\frac{m}{2} \log \left(\lambda_{i}\right)-\frac{1}{2} \log \left|\Omega_{i}\left(\rho_{1}\right)\right|-\frac{1}{2} \log \left|\Omega_{i}\left(\rho_{2}\right)\right|-\frac{1}{2} A_{i}
$$

e 
$A_{i}=\frac{\left(\left(\boldsymbol{Y}_{i}-\alpha \mathbf{1}\right)+\beta \boldsymbol{a}_{i} x_{i}\right)^{\top} \boldsymbol{\Omega}_{i}\left(\boldsymbol{\rho}_{1}\right)^{-1}\left(\left(\boldsymbol{Y}_{i}-\alpha \mathbf{1}\right)+\beta \boldsymbol{a}_{i} x_{i}\right)}{\lambda_{i}}+\frac{\left(\boldsymbol{X}_{i}-\boldsymbol{b}_{i} x_{i}\right)^{\top} \boldsymbol{\Omega}_{i}\left(\rho_{2}\right)^{-1}\left(\boldsymbol{X}_{i}-\boldsymbol{b}_{i} x_{i}\right)}{\kappa_{i}}$,

com o vetor $\boldsymbol{\theta}=(\alpha, \beta, \boldsymbol{\lambda}, \boldsymbol{\kappa}, \boldsymbol{x})^{\top}$.

$$
\frac{\partial \ell}{\partial x_{i}}=\boldsymbol{q}_{i x_{i}}
$$

sendo

$$
\begin{gathered}
\boldsymbol{q}_{i x_{i}}=-\frac{1}{2}\left\{\frac{-2 \beta \boldsymbol{a}_{i}^{\top} \boldsymbol{\Omega}_{i}\left(\boldsymbol{\rho}_{1}\right)^{-1}\left(\boldsymbol{Y}_{i}-\alpha \mathbf{1}\right)+2 \beta^{2} x_{i} \boldsymbol{a}_{i}^{\top} \boldsymbol{\Omega}_{i}\left(\rho_{1}\right)^{-1} \boldsymbol{a}_{i}}{\lambda_{i}}+\right. \\
\left.+\frac{2 x_{i} \boldsymbol{b}_{i}^{\top} \Omega_{i}\left(\rho_{2}\right)^{-1} \boldsymbol{b}_{i}-2 \boldsymbol{b}_{i}^{\top} \boldsymbol{\Omega}_{i}\left(\boldsymbol{\rho}_{2}\right)^{-1} \boldsymbol{X}_{i}}{\kappa_{i}}\right\} \\
\frac{\partial \ell}{\partial \alpha}=\sum_{i \in I} \boldsymbol{q}_{i \alpha},
\end{gathered}
$$

sendo $\boldsymbol{q}_{i \alpha}=\frac{\partial \ell_{i}}{\partial \alpha}=\frac{1}{\lambda_{i}}\left(\mathbf{1}^{\top} \boldsymbol{\Omega}_{i}\left(\rho_{1}\right)^{-1} \boldsymbol{Y}_{i}-\alpha \mathbf{1}^{\top} \boldsymbol{\Omega}_{i}\left(\rho_{1}\right)^{-1} \mathbf{1}-\beta x_{i} \boldsymbol{a}_{i}^{\top} \boldsymbol{\Omega}_{i}\left(\rho_{1}\right)^{-1} \mathbf{1}\right)$.

$$
\frac{\partial \ell}{\partial \beta}=\sum_{i \in I} \boldsymbol{q}_{i \beta}
$$

sendo $\boldsymbol{q}_{i \beta}=\frac{\partial \ell_{i}}{\partial \beta}=\frac{1}{\lambda_{i}}\left(x_{i} \boldsymbol{a}_{i}^{\top} \boldsymbol{\Omega}_{i}\left(\rho_{1}\right)^{-1} \boldsymbol{Y}_{i}-x_{i} \alpha \boldsymbol{a}_{i}^{\top} \boldsymbol{\Omega}_{i}\left(\rho_{1}\right)^{-1} \mathbf{1}-\beta x_{i}^{2} \boldsymbol{a}_{i}^{\top} \boldsymbol{\Omega}_{i}\left(\rho_{1}\right)^{-1} \boldsymbol{a}_{i}\right)$.

Fazendo $e_{i}=\boldsymbol{Y}_{i}-\alpha \mathbf{1}-\beta \boldsymbol{a}_{i} x_{i}, \boldsymbol{u}_{i}=\boldsymbol{X}_{i}-\boldsymbol{b}_{i} x_{i}$ e derivando em relação a $\lambda_{i}$ e $\kappa_{i}$ para todo $i=\{1, \ldots, n\}$, teremos

$$
\frac{\partial \ell}{\partial \lambda_{i}}=q_{i \lambda_{i}}
$$

sendo $\boldsymbol{q}_{i \lambda_{i}}=\frac{\partial \ell_{i}}{\partial \lambda_{i}}=-\frac{m}{2 \lambda_{i}}+\frac{1}{2}\left\{\frac{\boldsymbol{e}_{i}^{\top} \Omega_{i}\left(\rho_{1}\right)^{-1} e_{i}}{\lambda_{i}^{2}}\right\} \mathrm{e}$

$$
\frac{\partial \ell}{\partial \kappa_{i}}=q_{i \kappa_{i}}
$$

sendo $\boldsymbol{q}_{i \kappa_{i}}=\frac{\partial \ell_{i}}{\partial \kappa_{i}}=-\frac{m}{2 \kappa_{i}}+\frac{1}{2}\left\{\frac{\boldsymbol{u}_{i}^{\top} \Omega_{i}\left(\rho_{1}\right)^{-1} \boldsymbol{u}_{i}}{\kappa_{i}^{2}}\right\}$. 
Derivando em relação a $\rho_{1 j}$ e $\rho_{2 j}$ para todo $j=1, \ldots, p$ teremos

$$
\frac{\partial \ell}{\partial \rho_{1 j}}=\sum_{i=1}^{n} \boldsymbol{q}_{i \rho_{1 j}}
$$

sendo

$$
\begin{gathered}
\boldsymbol{q}_{i \rho_{1 j}}=-\frac{1}{2} \operatorname{tr}\left\{\Omega_{i}\left(\rho_{1}\right)^{-1} J_{1 j}\right\}+\frac{1}{2 \lambda_{i}} \operatorname{tr}\left\{\Omega_{i}\left(\rho_{1}\right)^{-1} J_{1 j} \Omega_{i}\left(\rho_{1}\right)^{-1} e_{i} e_{i}^{\top}\right\} . \\
\frac{\partial \ell}{\partial \rho_{2 j}}=\sum_{i=1}^{n} \boldsymbol{q}_{i \rho_{2 j}}, \\
\boldsymbol{q}_{i \rho_{2 j}}=-\frac{1}{2} \operatorname{tr}\left\{\Omega_{i}\left(\rho_{2}\right)^{-1} \boldsymbol{J}_{2 j}\right\}+\frac{1}{2 \kappa_{i}} \operatorname{tr}\left\{\Omega_{i}\left(\rho_{2}\right)^{-1} \boldsymbol{J}_{2 j} \Omega_{i}\left(\rho_{2}\right)^{-1} \boldsymbol{u}_{i} \boldsymbol{u}_{i}^{\top}\right\} .
\end{gathered}
$$

A matriz $\boldsymbol{J}_{k j}$, é a matriz de derivadas de $\boldsymbol{\Omega}_{i}\left(\boldsymbol{\rho}_{k}\right)$ em relação a $\rho_{k j}$ para $k=1,2$. Igualando (A.1)-(A.7) a zero obtemos os estimadores abaixo:

Caso 1: Dependência

$$
\begin{aligned}
& \hat{x}_{i}=\frac{\beta \kappa_{i} \boldsymbol{a}_{i}^{\top} \Omega_{i}\left(\rho_{1}\right)^{-1}\left(\boldsymbol{Y}_{i}-\alpha \mathbf{1}\right)+\lambda_{i} \boldsymbol{b}_{i}^{\top} \boldsymbol{\Omega}_{i}\left(\rho_{2}\right)^{-1} \boldsymbol{X}_{i}}{\beta^{2} \kappa_{i} \boldsymbol{a}_{i}^{\top} \Omega_{i}\left(\rho_{1}\right)^{-1} \boldsymbol{a}_{i}+\lambda_{i} \boldsymbol{b}_{i}^{\top} \Omega_{i}\left(\rho_{2}\right)^{-1} \boldsymbol{b}_{i}}, \quad i=1,2, \ldots, n \\
& \hat{\beta}=\frac{\sum_{i \in I} \omega_{i}\left(a_{i}\right) \hat{x}_{i}\left(\boldsymbol{Y}_{i}-\mathbf{1} \bar{Y}_{\boldsymbol{\omega}(\mathbf{1})}\right)}{\sum_{i \in I} \omega_{i}\left(a_{i}\right) \hat{x}_{i}\left(a_{i} \hat{x}_{i}-\mathbf{1} \hat{\bar{x}} \boldsymbol{\omega}(a)\right)}, \hat{\alpha}=\bar{Y}_{\boldsymbol{\omega}(\mathbf{1})}-\hat{\beta} \hat{\bar{x}} \boldsymbol{\omega}(\boldsymbol{a})
\end{aligned}
$$

sendo que

$$
\bar{Y}_{\boldsymbol{\omega}(\mathbf{1})}=\sum_{i \in I} \boldsymbol{\omega}_{i}(\mathbf{1}) \boldsymbol{Y}_{i}, \hat{\bar{x}}_{\boldsymbol{\omega}\left(\boldsymbol{a}_{i}\right)}=\sum_{i \in I} \hat{x}_{i} \boldsymbol{\omega}_{i}\left(\boldsymbol{a}_{i}\right)
$$

e

$$
\omega_{i}\left(a_{i}\right)=\frac{a_{i}^{\top} \Omega_{i}\left(\rho_{1}\right)^{-1}}{\lambda_{i} \sum_{i \in I}\left(1^{\top} \Omega_{i}\left(\rho_{1}\right)^{-1} 1 / \lambda_{i}\right)} .
$$


Os estimadores para $\lambda_{i}$ e $\kappa_{i}$ são dados por

$$
\hat{\lambda}_{i}=\frac{\hat{\boldsymbol{e}}_{i}^{\top} \boldsymbol{\Omega}_{i}\left(\hat{\boldsymbol{\rho}}_{1}\right)^{-1} \hat{\boldsymbol{e}}_{i}}{m} \text { e } \hat{\kappa}_{i}=\frac{\hat{\boldsymbol{u}}_{i}^{\top} \Omega_{i}\left(\hat{\boldsymbol{\rho}}_{2}\right)^{-1} \hat{u}_{i}}{m}
$$

Os estimadores para $\rho_{1}$ e $\rho_{2}$ são obtidos de forma iterativa, usando o algotimo de Escore de Fisher chegamos as seguintes expressões

$$
\begin{aligned}
& \hat{\boldsymbol{\rho}}_{1}^{(v+1)}=\hat{\boldsymbol{\rho}}_{1}^{(v)}+\left.\mathrm{E}\left[\boldsymbol{I}\left(\hat{\boldsymbol{\rho}}_{1}^{(v)}\right)\right]^{-1} \frac{\partial \ell}{\partial \rho_{1}}\right|_{\rho_{1 l}=\hat{\boldsymbol{\rho}}_{1}^{(v)}} \\
& \hat{\boldsymbol{\rho}}_{2}^{(v+1)}=\hat{\boldsymbol{\rho}}_{2}^{(v)}+\left.\mathrm{E}\left[\boldsymbol{I}\left(\hat{\boldsymbol{\rho}}_{2}^{(v)}\right)\right]^{-1} \frac{\partial \ell}{\partial \rho_{2}}\right|_{\rho_{2}=\hat{\boldsymbol{\rho}}_{2}^{(v)}}
\end{aligned}
$$

onde os elementos de $\mathrm{E}\left[\boldsymbol{I}\left(\hat{\boldsymbol{\rho}}_{1}^{(v)}\right)\right]$ e $\mathrm{E}\left[\boldsymbol{I}\left(\hat{\boldsymbol{\rho}}_{2}^{(v)}\right)\right]$ são dados em (A.8) - (A.11). Se exite correlação entre as repetições, mas a verdadeira concentração pode ser replicada identicamente, então os estimadores são obtidos fazendo $a_{i}=b_{i}=1$ nos estimadores do Caso 1. Na situação de independência das repetições, ou seja, $\Omega_{i}\left(\rho_{1}\right)=\Omega_{i}\left(\rho_{2}\right)=$ $\boldsymbol{I}$ e a verdadeira concentração é replicada com erro conhecido, os estimadores são dados por

\section{Caso 2: Independência}

$$
\begin{gathered}
\hat{x}_{i}=\frac{\beta \kappa_{i} \boldsymbol{a}_{i}^{\top}\left(\boldsymbol{Y}_{i}-\alpha \mathbf{1}\right)+\lambda_{i} \boldsymbol{b}_{i}^{\top} \boldsymbol{X}_{i}}{\beta^{2} \kappa_{i} \boldsymbol{a}_{i}^{\top} \boldsymbol{a}_{i}+\lambda_{i} \boldsymbol{b}_{i}^{\top} \boldsymbol{b}_{i}}, \quad i=1,2, \ldots, n \\
\hat{\beta}=\frac{\sum_{i \in I} \boldsymbol{\omega}_{i}\left(\boldsymbol{a}_{i}\right) \hat{x}_{i}\left(\boldsymbol{Y}_{i}-\mathbf{1} \bar{Y}_{\boldsymbol{\omega}(\mathbf{1})}\right)}{\sum_{i \in I} \boldsymbol{\omega}_{i}\left(\boldsymbol{a}_{i}\right) \hat{x}_{i}\left(\boldsymbol{a}_{i} \hat{x}_{i}-\mathbf{1} \hat{\bar{x}} \boldsymbol{\omega}(\boldsymbol{a})\right)}, \hat{\alpha}=\bar{Y}_{\boldsymbol{\omega}(\mathbf{1})}-\hat{\beta} \hat{\bar{x}} \boldsymbol{\omega}(\boldsymbol{a})
\end{gathered}
$$

sendo que

$$
\bar{Y}_{\boldsymbol{\omega}(\mathbf{1})}=\sum_{i \in I} \boldsymbol{\omega}_{i}(\mathbf{1}) Y_{i}, \hat{\bar{x}} \boldsymbol{\omega}\left(a_{i}\right)=\sum_{i \in I} \hat{x}_{i} \boldsymbol{\omega}_{i}\left(a_{i}\right)
$$

$\mathrm{e}$

$$
\boldsymbol{\omega}_{i}\left(\boldsymbol{a}_{i}\right)=\frac{\boldsymbol{a}_{i}^{\top}}{\lambda_{i} \sum_{i \in I}\left(m / \lambda_{i}\right)}
$$


Os estimadores para $\lambda_{i}$ e $\kappa_{i}$ são dados por

$$
\hat{\lambda}_{i}=\frac{\hat{\boldsymbol{e}}_{i}^{\top} \hat{\boldsymbol{e}}_{i}}{m} \text { e } \hat{\kappa}_{i}=\frac{\hat{\boldsymbol{u}}_{i}^{\top} \hat{\boldsymbol{u}}_{i}}{m}
$$

Se além de repetições independentes a verdadeira concentração puder ser replicada identicamente, voltamos ao caso mais simples e os estimadores são obtidos fazendo $a_{i}=b_{i}=1$ no Caso 2 .

As derivadas segundas são dadas logo abaixo

$$
\begin{aligned}
& \frac{\partial^{2} \ell}{\partial \alpha^{2}}=-\sum_{i \in I}\left(1^{\top} \Omega_{i}\left(\rho_{1}\right)^{-1} 1 / \lambda_{i}\right) \Rightarrow \mathrm{E}\left(-\frac{\partial^{2} \ell}{\partial \alpha^{2}}\right)=\sum_{i \in I}\left(1^{\top} \Omega_{i}\left(\rho_{1}\right)^{-1} 1 / \lambda_{i}\right) \\
& \frac{\partial^{2} \ell}{\partial \alpha \partial \beta}=-\sum_{i \in I}\left(\boldsymbol{a}_{i}^{\top} \boldsymbol{\Omega}_{i}\left(\rho_{1}\right)^{-1} \mathbf{1} x_{i} / \lambda_{i}\right) \Rightarrow \mathrm{E}\left(-\frac{\partial^{2} \ell}{\partial \alpha \partial \beta}\right)=\sum_{i \in I}\left(\boldsymbol{a}_{i}^{\top} \boldsymbol{\Omega}_{i}\left(\boldsymbol{\rho}_{1}\right)^{-1} \mathbf{1} x_{i} / \lambda_{i}\right) \\
& \frac{\partial^{2} \ell}{\partial \alpha \partial \lambda_{i}}=-\left(1 / \lambda_{i}^{2}\right) \mathbf{1}^{\top} \boldsymbol{\Omega}_{i}\left(\rho_{1}\right)^{-1}\left(\boldsymbol{Y}_{i}-\alpha \mathbf{1}-\beta \boldsymbol{a}_{i} x_{i}\right) \Rightarrow \mathrm{E}\left(-\frac{\partial^{2} \ell}{\partial \alpha \partial \lambda_{i}}\right)=0 \\
& \frac{\partial^{2} \ell}{\partial \alpha \partial \kappa_{i}}=0 \Rightarrow \mathrm{E}\left(-\frac{\partial^{2} \ell}{\partial \alpha \partial \kappa_{i}}\right)=0 \\
& \frac{\partial^{2} \ell}{\partial \alpha \partial x_{i}}=\beta \boldsymbol{a}_{i}^{\top} \Omega_{i}\left(\rho_{1}\right)^{-1} \mathbf{1} / \lambda_{i} \Rightarrow \mathrm{E}\left(-\frac{\partial^{2} \ell}{\partial \alpha \partial x_{i}}\right)=\beta \boldsymbol{a}_{i}^{\top} \boldsymbol{\Omega}_{i}\left(\rho_{1}\right)^{-1} \mathbf{1} / \lambda_{i} \\
& \frac{\partial^{2} \ell}{\partial \alpha \partial \rho_{1_{j}}}=-\sum_{i \in I} \frac{1}{\lambda_{i}}\left\{\operatorname{tr}\left[\Omega_{i}\left(\rho_{1}\right)^{-1} J_{1 j} \Omega_{i}\left(\rho_{1}\right)^{-1}\left(Y_{i} 1^{\top}-\alpha 11^{\top}-\beta x_{i} a_{i} 1^{\top}\right)\right]\right\} \\
& \Rightarrow E\left(-\frac{\partial^{2} \ell}{\partial \alpha \partial \rho_{1_{j}}}\right)=0 \\
& \frac{\partial^{2} \ell}{\partial \alpha \partial \rho_{2_{j}}}=0 \Rightarrow E\left(-\frac{\partial^{2} \ell}{\partial \alpha \partial \rho_{2_{j}}}\right)=0
\end{aligned}
$$




$$
\begin{aligned}
& \frac{\partial^{2} \ell}{\partial \beta^{2}}=-\sum_{i \in I}\left(\boldsymbol{a}_{i}^{\top} \boldsymbol{\Omega}_{i}\left(\rho_{1}\right)^{-1} \boldsymbol{a}_{i} x_{i}^{2} / \lambda_{i}\right) \Rightarrow \mathrm{E}\left(-\frac{\partial^{2} \ell}{\partial \beta^{2}}\right)=\sum_{i \in I}\left(\boldsymbol{a}_{i}^{\top} \Omega_{i}\left(\boldsymbol{\rho}_{1}\right)^{-1} \boldsymbol{a}_{i} x_{i}^{2} / \lambda_{i}\right) \\
& \frac{\partial^{2} \ell}{\partial \beta \partial \lambda_{i}}=-\left(1 / \lambda_{i}^{2}\right) x_{i} \boldsymbol{a}_{i}^{\top} \boldsymbol{\Omega}_{i}\left(\boldsymbol{\rho}_{1}\right)^{-1}\left(\boldsymbol{Y}_{i}-\alpha \mathbf{1}-\beta \boldsymbol{a}_{i} x_{i}\right) \Rightarrow \mathrm{E}\left(-\frac{\partial^{2} \ell}{\partial \beta \partial \lambda_{i}}\right)=0 \\
& \frac{\partial^{2} \ell}{\partial \beta \partial \kappa_{i}}=0 \Rightarrow \mathrm{E}\left(-\frac{\partial^{2} \ell}{\partial \beta \partial \kappa_{i}}\right)=0 \\
& \frac{\partial^{2} \ell}{\partial \beta \partial x_{i}}=\frac{1}{\lambda_{i}}\left(\boldsymbol{a}_{i}^{\top} \boldsymbol{\Omega}_{i}\left(\boldsymbol{\rho}_{1}\right)^{-1} \boldsymbol{Y}_{i}-\alpha \boldsymbol{a}_{i}^{\top} \boldsymbol{\Omega}_{i}\left(\boldsymbol{\rho}_{1}\right)^{-1} \mathbf{1}-2 \beta x_{i} \boldsymbol{a}_{i}^{\top} \boldsymbol{\Omega}_{i}\left(\rho_{1}\right)^{-1} \boldsymbol{a}_{i}\right) \\
& \Rightarrow \mathrm{E}\left(-\frac{\partial^{2} \ell}{\partial \beta \partial \kappa_{i}}\right)=\frac{1}{\lambda_{i}} \beta x_{i} \boldsymbol{a}_{i}^{\top} \Omega_{i}\left(\rho_{1}\right)^{-1} \boldsymbol{a}_{i} \\
& \frac{\partial^{2} \ell}{\partial \beta \partial \rho_{1_{j}}}=-\sum_{i \in I} \frac{x_{i}}{\lambda_{i}}\left\{\operatorname{tr}\left[\boldsymbol{\Omega}_{i}\left(\rho_{1}\right)^{-1} \boldsymbol{J}_{1 j} \boldsymbol{\Omega}_{i}\left(\rho_{1}\right)^{-1}\left(\boldsymbol{Y}_{i} \mathbf{1}^{\top}-\alpha \mathbf{1} \mathbf{1}^{\top}-\beta x_{i} \boldsymbol{a}_{i} \mathbf{1}^{\top}\right)\right]\right\} \\
& \Rightarrow \mathrm{E}\left(-\frac{\partial^{2} \ell}{\partial \beta \partial \rho_{1_{j}}}\right)=0 \\
& \frac{\partial^{2} \ell}{\partial \beta \partial \rho_{2_{j}}}=0 \Rightarrow \mathrm{E}\left(-\frac{\partial^{2} \ell}{\partial \beta \partial \rho_{2_{j}}}\right)=0 \\
& \frac{\partial^{2} \ell}{\partial \lambda_{i}^{2}}=-\left(1 / \lambda_{i}^{3}\right) \boldsymbol{e}_{i}^{\top} \boldsymbol{\Omega}_{i}\left(\rho_{1}\right)^{-1} \boldsymbol{e}_{i}+m /\left(2 \lambda_{i}^{2}\right) \Rightarrow \mathrm{E}\left(-\frac{\partial^{2} \ell}{\partial \lambda_{i}^{2}}\right)=m /\left(2 \lambda_{i}^{2}\right) \\
& \frac{\partial^{2} \ell}{\partial \lambda_{i} \partial \kappa_{i}}=0 \Rightarrow E\left(-\frac{\partial^{2} \ell}{\partial \lambda_{i} \partial \kappa_{i}}\right)=0 \\
& \frac{\partial^{2} \ell}{\partial \lambda_{i} \partial x_{i}}=\frac{1}{2 \lambda_{i}^{2}}\left[-2 \beta \boldsymbol{a}_{i}^{\top} \Omega_{i}\left(\rho_{1}\right)^{-1}\left(Y_{i}-\alpha 1\right)+2 \beta^{2} x_{i} \boldsymbol{a}_{i}^{\top} \boldsymbol{\Omega}_{i}\left(\rho_{1}\right)^{-1} \boldsymbol{a}_{i}\right]
\end{aligned}
$$




$$
\begin{aligned}
& \Rightarrow \mathrm{E}\left(-\frac{\partial^{2} \ell}{\partial \lambda_{i} \partial x_{i}}\right)=0 \\
& \frac{\partial^{2} \ell}{\partial \lambda_{i} \partial \rho_{1_{j}}}=-\frac{1}{2 \lambda_{i}^{2}} \operatorname{tr}\left[\Omega_{i}\left(\rho_{1}\right)^{-1} \boldsymbol{J}_{1 j} \Omega_{i}\left(\rho_{1}\right)^{-1} \boldsymbol{e}_{i} \boldsymbol{e}_{i}^{\top}\right] \Rightarrow \mathrm{E}\left(-\frac{\partial^{2} \ell}{\partial \lambda_{i} \partial \rho_{1_{j}}}\right)=\frac{1}{2 \lambda_{i}} \operatorname{tr}\left[\Omega_{i}\left(\rho_{1}\right)^{-1} \boldsymbol{J}_{1 j}\right] \\
& \frac{\partial^{2} \ell}{\partial \lambda_{i} \partial \rho_{2_{j}}}=0 \Rightarrow \mathrm{E}\left(-\frac{\partial^{2} \ell}{\partial \lambda_{i} \partial \rho_{2_{j}}}\right)=0 \\
& \frac{\partial^{2} \ell}{\partial \kappa_{i}^{2}}=-\left(1 / \kappa_{i}^{3}\right) \boldsymbol{u}_{i}^{\top} \boldsymbol{\Omega}_{i}\left(\boldsymbol{\rho}_{\mathbf{2}}\right)^{-1} \boldsymbol{u}_{i}+m /\left(2 \kappa_{i}^{2}\right) \Rightarrow \mathrm{E}\left(-\frac{\partial^{2} \ell}{\partial \kappa_{i}^{2}}\right)=m /\left(2 \kappa_{i}^{2}\right) \\
& \frac{\partial^{2} \ell}{\partial \kappa_{i} \partial x_{i}}=\frac{1}{2 \kappa_{i}^{2}}\left[2 x_{i} \boldsymbol{b}_{i}^{\top} \boldsymbol{\Omega}_{i}\left(\boldsymbol{\rho}_{2}\right)^{-1} \boldsymbol{b}_{i}-2 \boldsymbol{b}_{i}^{\top} \boldsymbol{\Omega}_{i}\left(\boldsymbol{\rho}_{2}\right)^{-1} \boldsymbol{X}_{i}\right] \\
& \Rightarrow \mathrm{E}\left(-\frac{\partial^{2} \ell}{\partial \kappa_{i} \partial x_{i}}\right)=0 \\
& \frac{\partial^{2} \ell}{\partial \kappa_{i} \partial \rho_{1_{j}}}=0 \Rightarrow \mathrm{E}\left(-\frac{\partial^{2} \ell}{\partial \kappa_{i} \partial \rho_{1_{j}}}\right)=0 \\
& \frac{\partial^{2} \ell}{\partial \kappa_{i} \partial \rho_{2_{j}}}=-\frac{1}{2 \kappa_{i}^{2}} \operatorname{tr}\left[\Omega_{i}\left(\rho_{2}\right)^{-1} \boldsymbol{J}_{2 j} \Omega_{i}\left(\rho_{2}\right)^{-1} \boldsymbol{u}_{i} \boldsymbol{u}_{i}^{\top}\right] \Rightarrow \mathrm{E}\left(-\frac{\partial^{2} \ell}{\partial \kappa_{i} \partial \rho_{2_{j}}}\right)=\frac{1}{2 \kappa_{i}} \operatorname{tr}\left[\Omega_{i}\left(\rho_{2}\right)^{-1} \boldsymbol{J}_{2 j}\right] \\
& \frac{\partial^{2} \ell}{\partial \rho_{1_{j}}^{2}}=-\frac{1}{2} \sum_{i=1}^{n} \operatorname{tr}\left[\Omega_{i}\left(\rho_{1}\right)^{-1} \boldsymbol{J}_{1 j} \Omega_{i}\left(\rho_{1}\right)^{-1} \boldsymbol{J}_{1 j}\right] \\
& \Rightarrow \mathrm{E}\left(-\frac{\partial^{2} \ell}{\partial \rho_{1_{j}}^{2}}\right)=\frac{1}{2} \sum_{i=1}^{n} \operatorname{tr}\left[\Omega_{i}\left(\rho_{1}\right)^{-1} J_{1 j} \Omega_{i}\left(\rho_{1}\right)^{-1} J_{1 j}\right] \\
& \frac{\partial^{2} \ell}{\partial \rho_{1_{1}} \partial \rho_{1_{j_{2}}}}=-\frac{1}{2} \sum_{i=1}^{n} \operatorname{tr}\left[\Omega_{i}\left(\rho_{1}\right)^{-1} \boldsymbol{J}_{1 j_{1}} \Omega_{i}\left(\rho_{1}\right)^{-1} J_{1 j_{1}}\right] \\
& \Rightarrow \mathrm{E}\left(-\frac{\partial^{2} \ell}{\partial \rho_{1_{j_{1}}} \partial \rho_{1_{j_{2}}}}\right)=\frac{1}{2} \sum_{i=1}^{n} \operatorname{tr}\left[\Omega_{i}\left(\rho_{1}\right)^{-1} \boldsymbol{J}_{1 j_{1}} \Omega_{i}\left(\rho_{1}\right)^{-1} \boldsymbol{J}_{1 j_{1}}\right] \\
& \frac{\partial^{2} \ell}{\partial \rho_{2_{j}}^{2}}=-\frac{1}{2} \sum_{i=1}^{n} \operatorname{tr}\left[\Omega_{i}\left(\rho_{2}\right)^{-1} \boldsymbol{J}_{2 j} \Omega_{i}\left(\rho_{2}\right)^{-1} \boldsymbol{J}_{2 j}\right]
\end{aligned}
$$




$$
\begin{array}{r}
\Rightarrow \mathrm{E}\left(-\frac{\partial^{2} \ell}{\partial \rho_{2_{j}}^{2}}\right)=\frac{1}{2} \sum_{i=1}^{n} \operatorname{tr}\left[\Omega_{i}\left(\rho_{2}\right)^{-1} \boldsymbol{J}_{2 j} \Omega_{i}\left(\rho_{2}\right)^{-1} \boldsymbol{J}_{2 j}\right] \\
\frac{\partial^{2} \ell}{\partial \rho_{2_{j_{1}}} \partial \rho_{2_{j_{2}}}}=-\frac{1}{2} \sum_{i=1}^{n} \operatorname{tr}\left[\Omega_{i}\left(\boldsymbol{\rho}_{2}\right)^{-1} \boldsymbol{J}_{2 j_{1}} \Omega_{i}\left(\rho_{2}\right)^{-1} \boldsymbol{J}_{2 j_{1}}\right] \\
\Rightarrow \mathrm{E}\left(-\frac{\partial^{2} \ell}{\partial \rho_{2_{j_{1}}} \partial \rho_{{j_{2}}_{2}}}\right)=\frac{1}{2} \sum_{i=1}^{n} \operatorname{tr}\left[\Omega_{i}\left(\boldsymbol{\rho}_{2}\right)^{-1} \boldsymbol{J}_{2 j_{1}} \Omega_{i}\left(\boldsymbol{\rho}_{2}\right)^{-1} \boldsymbol{J}_{2 j_{1}}\right] \\
\frac{\partial^{2} \ell}{\partial x_{i}^{2}}=-\frac{1}{2}\left[2 \beta^{2} \boldsymbol{a}_{i}^{\top} \boldsymbol{\Omega}_{i}\left(\boldsymbol{\rho}_{1}\right)^{-1} \boldsymbol{a}_{i} / \lambda_{i}+2 \boldsymbol{b}_{i}^{\top} \boldsymbol{\Omega}_{i}\left(\boldsymbol{\rho}_{2}\right)^{-1} \boldsymbol{b}_{i} / \kappa_{i}\right] \\
\Rightarrow \mathrm{E}\left(-\frac{\partial^{2} \ell}{\partial x_{i}^{2}}\right)=\frac{\beta^{2} \kappa_{i} \boldsymbol{a}_{i}^{\top} \boldsymbol{\Omega}_{i}\left(\boldsymbol{\rho}_{1}\right)^{-1} \boldsymbol{a}_{i}+\lambda_{i} \boldsymbol{b}_{i}^{\top} \Omega_{i}\left(\boldsymbol{\rho}_{2}\right)^{-1} \boldsymbol{b}_{i}}{\lambda_{i} \kappa_{i}}
\end{array}
$$

Caso exista independência entre as réplicas, todas as derivadas acima são válidas, basta apenas tomar $\Omega_{i}\left(\rho_{1}\right)=\Omega_{i}\left(\rho_{2}\right)=\boldsymbol{I}$ (matriz identidade) e retirar as derivadas em relação a $\rho_{1}$ e $\rho_{2}$. Se não existir erro na replicação da verdadeira concentração, basta tomar $\boldsymbol{a}_{i}=\boldsymbol{b}_{i}=1$. A inversa da matriz de covariâncias para o vetor $\hat{\boldsymbol{\theta}}$ é dada por

$$
\Pi=\left[\begin{array}{lll}
\boldsymbol{A} & \mathbf{0}_{2 \times 2(n+p)} & \boldsymbol{B} \\
\mathbf{0}_{2(n+p) \times 2} & \boldsymbol{C} & \mathbf{0}_{2(n+p) \times n} \\
\boldsymbol{B}^{\top} & \mathbf{0}_{n \times 2(n+p)} & \boldsymbol{D}
\end{array}\right]
$$

sendo

$$
\begin{gathered}
\boldsymbol{A}=\left[\begin{array}{cc}
\sum_{i \in I}\left(\mathbf{1}^{\top} \boldsymbol{\Omega}_{i}\left(\boldsymbol{\rho}_{1}\right)^{-1} \mathbf{1} / \lambda_{i}\right) & \sum_{i \in I}\left(\boldsymbol{a}_{i}^{\top} \boldsymbol{\Omega}_{i}\left(\rho_{1}\right)^{-1} \mathbf{1} x_{i} / \lambda_{i}\right) \\
\sum_{i \in I}\left(\boldsymbol{a}_{i}^{\top} \Omega_{i}\left(\rho_{1}\right)^{-1} 1 x_{i} / \lambda_{i}\right) & \sum_{i \in I}\left(\boldsymbol{a}_{i}^{\top} \boldsymbol{\Omega}_{i}\left(\rho_{1}\right)^{-1} \boldsymbol{a}_{i} x_{i}^{2} / \lambda_{i}\right)
\end{array}\right], \\
\boldsymbol{B}=\beta\left[\begin{array}{lll}
\boldsymbol{a}_{1}^{\top} \boldsymbol{\Omega}_{i}\left(\rho_{1}\right)^{-1} 1 / \lambda_{1} & \ldots & \boldsymbol{a}_{n}^{\top} \boldsymbol{\Omega}_{i}\left(\rho_{1}\right)^{-1} \mathbf{1} / \lambda_{n} \\
x_{1} \boldsymbol{a}_{1}^{\top} \Omega_{i}\left(\rho_{1}\right)^{-1} \boldsymbol{a}_{1} / \lambda_{1} & \ldots & x_{n} \boldsymbol{a}_{n}^{\top} \boldsymbol{\Omega}_{i}\left(\rho_{1}\right)^{-1} \boldsymbol{a}_{n} / \lambda_{n}
\end{array}\right],
\end{gathered}
$$

$C$ é a matriz de informação esperada de Fisher para os parâmetros $\left(\lambda, \kappa, \rho_{1}, \rho_{2}\right)$, não será necessário explicita-la, pois veremos que a matriz de covariâncias de $(\hat{\alpha}, \hat{\beta})$ 
não depende dessa matriz. Se houver independência das réplicas basta fazer $p=0$ na matriz (A.12)

e

$\boldsymbol{D}=\operatorname{diag}\left(\frac{\beta^{2} \kappa_{1} \boldsymbol{a}_{1}^{\top} \Omega_{i}\left(\rho_{1}\right)^{-1} \boldsymbol{a}_{1}+\lambda_{1} \boldsymbol{b}_{1}^{\top} \Omega_{i}\left(\rho_{2}\right)^{-1} \boldsymbol{b}_{1}}{\lambda_{1} \kappa_{1}}, \ldots, \frac{\beta^{2} \kappa_{n} \boldsymbol{a}_{n}^{\top} \Omega_{i}\left(\rho_{1}\right)^{-1} \boldsymbol{a}_{n}+\lambda_{n} \boldsymbol{b}_{n}^{\top} \Omega_{i}\left(\rho_{2}\right)^{-1} \boldsymbol{b}_{n}}{\lambda_{n} \kappa_{n}}\right)$

Poderemos particionar a matriz $\Pi$ da seguinte forma

$$
\Pi=\left[\begin{array}{ll}
\Pi_{11} & \Pi_{12} \\
\Pi_{12}^{\top} & \Pi_{22}
\end{array}\right]
$$

sendo

$$
\Pi_{11}=\left[\begin{array}{ll}
\boldsymbol{A} & \mathbf{0}_{2 \times 2(n+p)} \\
\mathbf{0}_{2(n+p) \times 2} & \boldsymbol{C}
\end{array}\right] \quad, \quad \boldsymbol{\Pi}_{12}^{\top}=\left[\begin{array}{ll}
\boldsymbol{B}^{\top} & \mathbf{0}_{n \times 2(n+p)}
\end{array}\right] \quad \text { e } \quad \boldsymbol{\Pi}_{22}=\boldsymbol{D}
$$

Para encontrarmos a matriz de covariâncias de $(\hat{\alpha}, \hat{\beta})$, precisamos inverter a matriz $\Pi$ e depois selecionar a submatriz referente as duas primeiras linha e duas primeiras colunas, para isso definimos a inversa da matriz $\Pi$ da seguinte forma

$$
\Pi^{-1}=\left[\begin{array}{ll}
\Pi_{11}^{*} & \Pi_{12}^{*} \\
\Pi_{12}^{* \top} & \Pi_{22}^{*}
\end{array}\right]
$$

Estamos interessado somente em $\Pi_{11}^{*}$, fazendo as devidas multiplicações matriciais chegamos a expressão

$$
\Pi_{11}^{*}=\left[\Pi_{11}-\Pi_{12} \Pi_{22}^{-1} \Pi_{12}^{\top}\right]^{-1}
$$




$$
\begin{aligned}
\Pi_{11}^{*} & =\left\{\left[\begin{array}{ll}
A & 0_{2 \times 2(n+p)} \\
0_{2(n+p) \times 2} & C
\end{array}\right]-\left[\begin{array}{l}
B \\
0_{2(n+p) \times n}
\end{array}\right] D^{-1}\left[\begin{array}{ll}
B^{\top} & 0_{n \times 2(n+p)}
\end{array}\right]\right\}^{-1} \\
& =\left\{\left[\begin{array}{ll}
A & 0_{2 \times 2(n+p)} \\
0_{2(n+p) \times 2} & C
\end{array}\right]-\left[\begin{array}{ll}
B D^{-1} B^{\top} & 0_{2 \times 2(n+p)} \\
0_{2(n+p) \times 2} & 0_{2(n+p) \times 2(n+p)}
\end{array}\right]\right\}^{-1} \\
& =\left[\begin{array}{ll}
A-B D^{-1} B^{\top} & 0_{2 \times 2(n+p)} \\
0_{2(n+p) \times 2} & C
\end{array}\right]^{-1}
\end{aligned}
$$

para facilitar a notação fazemos

$\boldsymbol{B} \boldsymbol{D}^{-1} \boldsymbol{B}^{\top}=\sum_{i \in I}\left[\begin{array}{ll}\frac{\mathbf{1}^{\top} \boldsymbol{\Omega}_{i}\left(\boldsymbol{\rho}_{1}\right)^{-1} \mathbf{1}-\zeta_{i}^{*}\left(\mathbf{1}, \mathbf{1}, \boldsymbol{a}_{i}, \boldsymbol{b}_{i}\right)}{\lambda_{i}} & \frac{x_{i}\left(\mathbf{1}^{\top} \Omega_{i}\left(\boldsymbol{\rho}_{1}\right)^{-1} \boldsymbol{a}_{i}-\zeta_{i}^{*}\left(\mathbf{1}, \boldsymbol{a}_{i}, \boldsymbol{a}_{i}, \boldsymbol{b}_{i}\right)\right)}{\lambda_{i}} \\ \frac{x_{i}\left(\mathbf{1}^{\top} \Omega_{i}\left(\boldsymbol{\rho}_{1}\right)^{-1} \boldsymbol{a}_{i}-\zeta_{i}^{*}\left(\mathbf{1}, \boldsymbol{a}_{i}, \boldsymbol{a}_{i}, \boldsymbol{b}_{i}\right)\right)}{\lambda_{i}} & \frac{x_{i}^{2}\left(\boldsymbol{a}_{i}^{\top} \Omega_{i}\left(\rho_{1}\right)^{-1} \boldsymbol{a}_{i}-\zeta_{i}^{*}\left(\boldsymbol{a}_{i}, \boldsymbol{a}_{i}, \boldsymbol{a}_{i}, \boldsymbol{b}_{i}\right)\right)}{\lambda_{i}}\end{array}\right]$

sendo $\zeta_{i}^{*}\left(\boldsymbol{q}_{1}, \boldsymbol{q}_{2}, \boldsymbol{q}_{3}, \boldsymbol{q}_{4}\right)=\boldsymbol{q}_{1}^{\top} \Omega_{i}\left(\rho_{1}\right)^{-1} \boldsymbol{q}_{2}-\frac{\beta^{2} \kappa_{i}\left(\boldsymbol{q}_{3}^{\top} \Omega_{i}\left(\rho_{1}\right)^{-1} \boldsymbol{q}_{1}\right)\left(\boldsymbol{q}_{3}^{\top} \Omega_{i}\left(\rho_{1}\right)^{-1} \boldsymbol{q}_{2}\right)}{\beta^{2} \kappa_{i} \boldsymbol{q}_{3}^{\top} \Omega_{i}\left(\rho_{1}\right)^{-1} \boldsymbol{q}_{3}+\lambda_{i} \boldsymbol{q}_{4}^{\top} \Omega_{i}\left(\rho_{2}\right)^{-1} \boldsymbol{q}_{4}}$, uma função dos vetores $\boldsymbol{q}_{1}, \boldsymbol{q}_{2}, \boldsymbol{q}_{3}, \boldsymbol{q}_{4}$. Assim, fazendo a subtração $\boldsymbol{A}-\boldsymbol{B} \boldsymbol{D}^{-1} \boldsymbol{B}^{\top}$ teremos que a matriz de covariâncias de $(\hat{\alpha}, \hat{\beta})$ dada por

$$
\operatorname{Cov}(\hat{\alpha}, \hat{\beta})=\left[\begin{array}{cc}
\sum_{i \in I} \frac{\zeta_{i}^{*}\left(\mathbf{1}, \mathbf{1}, \boldsymbol{a}_{i}, \boldsymbol{b}_{i}\right)}{\lambda_{i}} & \sum_{i \in I} \frac{x_{i} \zeta_{i}^{*}\left(\mathbf{1}, \boldsymbol{a}_{i}, \boldsymbol{a}_{i}, \boldsymbol{b}_{i}\right)}{\lambda_{i}} \\
\sum_{i \in I} \frac{x_{i} \zeta_{i}^{*}\left(\mathbf{1}, \boldsymbol{a}_{i}, \boldsymbol{a}_{i}, \boldsymbol{b}_{i}\right)}{\lambda_{i}} & \sum_{i \in I} \frac{x_{i}^{2} \zeta_{i}^{*}\left(\boldsymbol{a}_{i}, \boldsymbol{a}_{i}, \boldsymbol{a}_{i}, \boldsymbol{b}_{i}\right)}{\lambda_{i}}
\end{array}\right]^{-1}
$$

Se $\Omega_{i}\left(\rho_{2}\right)=\Omega_{i}\left(\rho_{1}\right)=I$ (identidade) a matriz de covariâncias de $(\hat{\alpha}, \hat{\beta})$ é dada substituindo $\zeta_{i}^{*}\left(\boldsymbol{q}_{1}, \boldsymbol{q}_{2}, \boldsymbol{q}_{3}, \boldsymbol{q}_{4}\right)$ por $\zeta_{i}\left(\boldsymbol{q}_{1}, \boldsymbol{q}_{2}, \boldsymbol{q}_{3}, \boldsymbol{q}_{4}\right)=\boldsymbol{q}_{1}^{\top} \boldsymbol{q}_{2}-\frac{\beta^{2} \kappa_{i}\left(\boldsymbol{q}_{3}^{\top} \boldsymbol{q}_{1}\right)\left(\boldsymbol{q}_{3}^{\top} \boldsymbol{q}_{2}\right)}{\beta^{2} \kappa_{i} \boldsymbol{q}_{3}^{\top} \boldsymbol{q}_{3}+\lambda_{i} \boldsymbol{q}_{4}^{\top} \boldsymbol{q}_{4}}$. Se além de não houver correlação entre as réplicas $\boldsymbol{a}_{i}=\boldsymbol{b}_{i}=\mathbf{1}$ para todo $i=1,2, \ldots, n$ teremos

$$
\operatorname{Cov}(\hat{\alpha}, \hat{\beta})=\left[\begin{array}{cc}
\sum_{i \in I} \frac{\zeta_{i}(1,1,1,1)}{\lambda_{i}} & \sum_{i \in I} \frac{x_{i} \zeta_{i}(1,1,1,1)}{\lambda_{i}} \\
\sum_{i \in I} \frac{x_{i} \zeta_{i}(1,1,1,1)}{\lambda_{i}} & \sum_{i \in I} \frac{x_{i}^{2} \zeta_{i}(1,1,1,1)}{\lambda_{i}}
\end{array}\right]^{-1}
$$


$\operatorname{assim} \zeta_{i}(1,1,1,1)=m\left(1-\frac{\beta^{2} \kappa_{i}}{\beta^{2} \kappa_{i}+\lambda_{i}}\right)=c_{i}$ e chegamos ao caso mais simples. No caso em que $\boldsymbol{a}_{i}=\boldsymbol{b}_{i}=1$ para todo $i=1,2, \ldots, n$ mas existe correlação entre as observações, então a matriz de covariâncias será dada por

$$
\operatorname{Cov}(\hat{\alpha}, \hat{\beta})=\left[\begin{array}{cc}
\sum_{i \in I} \frac{\zeta_{i}^{*}(\mathbf{1}, \mathbf{1}, \mathbf{1}, \mathbf{1})}{\lambda_{i}} & \sum_{i \in I} \frac{x_{i} \zeta_{i}^{*}(\mathbf{1}, \mathbf{1}, \mathbf{1}, \mathbf{1})}{\lambda_{i}} \\
\sum_{i \in I} \frac{x_{i} \zeta_{i}^{*}(\mathbf{1}, \mathbf{1}, \mathbf{1}, \mathbf{1})}{\lambda_{i}} & \sum_{i \in I} \frac{x_{i}^{2} \zeta_{i}^{*}(\mathbf{1}, \mathbf{1}, \mathbf{1}, \mathbf{1})}{\lambda_{i}}
\end{array}\right]^{-1}
$$

sendo $\zeta_{i}^{*}(\mathbf{1}, \mathbf{1}, \mathbf{1}, \mathbf{1})=\left(\mathbf{1}^{\top} \Omega_{i}\left(\rho_{1}\right)^{-1} \mathbf{1}\right)\left\{1-\frac{\beta^{2} \mathbf{1}^{\top} \Omega_{i}\left(\rho_{1}\right)^{-1} \mathbf{1} \kappa_{i}}{\beta^{2} \mathbf{1}^{\top} \Omega_{i}\left(\rho_{1}\right)^{-1} \mathbf{1} \kappa_{i}+\mathbf{1}^{\top} \Omega_{i}\left(\rho_{2}\right)^{-1} \mathbf{1} \lambda_{i}}\right\}=$ $c_{i}^{*}$

Finalmente, se existe correlação entre as observações, $\boldsymbol{a}_{i}=a_{i} \mathbf{1}$ e $\boldsymbol{b}_{i}=b_{i} \mathbf{1}$ a matriz de covariâncias é dada por

$$
\operatorname{Cov}(\hat{\alpha}, \hat{\beta})=\left[\begin{array}{ll}
\sum_{i \in I} \frac{\zeta_{i}^{*}\left(\mathbf{1}, \mathbf{1}, a_{i} \mathbf{1}, b_{i} \mathbf{1}\right)}{\lambda_{i}} & \sum_{i \in I} \frac{x_{i} \zeta_{i}^{*}\left(\mathbf{1}, a_{i} \mathbf{1}, a_{i} \mathbf{1}, b_{i} \mathbf{1}\right)}{\lambda_{i}} \\
\sum_{i \in I} \frac{x_{i} \zeta_{i}^{*}\left(\mathbf{1}, a_{i} \mathbf{1}, a_{i} \mathbf{1}, b_{i} \mathbf{1}\right)}{\lambda_{i}} & \sum_{i \in I} \frac{x_{i}^{2} \zeta_{i}^{*}\left(a_{i} 1, a_{i} 1, a_{i} \mathbf{1}, b_{i} \mathbf{1}\right)}{\lambda_{i}}
\end{array}\right]^{-1}
$$




\section{A.2 Vários métodos}

No caso em que o interesse é verificar se vários métodos de mensuração estão calibrados com um método padrão, observaremos os vetores $\left\{\left(\boldsymbol{Y}_{11}, \boldsymbol{Y}_{12} \ldots, \boldsymbol{Y}_{1 r}, \boldsymbol{X}_{1}\right), \ldots,\left(\boldsymbol{Y}_{n 1}, \boldsymbol{Y}_{n 2} \ldots, \boldsymbol{Y}_{n r}, \boldsymbol{X}_{n}\right)\right\}$. Supondo que todos os instrumentos são mutuamente independentes e assumindo erros normais teremos

$$
\left(\begin{array}{c}
\boldsymbol{Y}_{i l} \\
\boldsymbol{X}_{i}
\end{array}\right) \sim N_{2 m}\left[\left(\begin{array}{c}
\left(\alpha_{l}+\beta_{l} x_{i}\right) \mathbf{1} \\
x_{i} \mathbf{1}
\end{array}\right) ;\left(\begin{array}{cc}
\lambda_{i l} \Omega_{i l}\left(\rho_{1 l}\right) & \mathbf{0} \\
\mathbf{0}^{\top} & \kappa_{i} \Omega_{i}\left(\rho_{2}\right)
\end{array}\right)\right] \forall i \in I .
$$

$\mathrm{O}$ vetor de parâmetros é $\boldsymbol{\theta}=\left(\boldsymbol{\alpha}, \boldsymbol{\beta}, \boldsymbol{\lambda}, \boldsymbol{\kappa}, \boldsymbol{\rho}_{\mathbf{1}}, \boldsymbol{\rho}_{2}, \boldsymbol{x}\right)$, sendo $\boldsymbol{\alpha}=\left(\alpha_{1}, \ldots, \alpha_{r}\right)$, $\boldsymbol{\beta}=\left(\beta_{1}, \ldots, \beta_{r}\right), \boldsymbol{x}=\left(x_{1}, \ldots, x_{n}\right), \boldsymbol{\kappa}=\left(\kappa_{11}, \ldots, \kappa_{n r}\right), \boldsymbol{\lambda}=\left(\lambda_{11}, \ldots, \lambda_{n r}\right)$, $\rho_{1 l}=\left(\rho_{11 l}, \rho_{12 l}, \ldots, \rho_{1 p l}\right)$ e $\rho_{2}=\left(\rho_{21}, \rho_{22}, \ldots, \rho_{2 p}\right)$. Assim o logaritmo da função de verossimilhança é dado por

$$
\ell(\theta)=\sum_{i \in I} \ell_{i}(\theta)
$$

sendo

$$
\begin{aligned}
\ell_{i}(\boldsymbol{\theta})= & -\frac{(r+2 m)}{2} \log (2 \pi)-\frac{m}{2} \log \left(\kappa_{i}\right)-\frac{1}{2} \log \left|\Omega_{i}\left(\rho_{2}\right)\right|-\frac{m}{2} \sum_{l=1}^{r} \log \left(\lambda_{i l}\right)- \\
& -\frac{1}{2} \sum_{l=1}^{r} \log \left|\Omega_{i l}\left(\rho_{1 l}\right)\right|-\frac{1}{2} A_{i}
\end{aligned}
$$

e

$$
\begin{gathered}
A_{i}=\sum_{l=1}^{r}\left\{\frac{\left(\boldsymbol{Y}_{i l}-\left(\alpha_{l}+\beta_{l} x_{i}\right) \mathbf{1}\right)^{\top} \Omega_{i l}\left(\rho_{1 l}\right)^{-1}\left(\boldsymbol{Y}_{i l}-\left(\alpha_{l}+\beta_{l} x_{i}\right) \mathbf{1}\right)}{\lambda_{i l}}\right\} \\
+\frac{\left(\boldsymbol{X}_{i}-x_{i} 1\right)^{\top} \boldsymbol{\Omega}_{i}\left(\rho_{2}\right)^{-1}\left(\boldsymbol{X}_{i}-x_{i} \mathbf{1}\right)}{\kappa_{i}} \\
\frac{\partial \ell}{\partial x_{i}}=\boldsymbol{q}_{i x_{i}}
\end{gathered}
$$


sendo

$$
\begin{gathered}
\boldsymbol{q}_{i x_{i}}=-\frac{1}{2}\left\{\frac{-2 \beta \sum_{l=1}^{r} \mathbf{1}^{\top} \Omega_{i l}\left(\rho_{1 l}\right)^{-1}\left(\boldsymbol{Y}_{i l}-\alpha_{l} \mathbf{1}+2 \beta_{l}^{2} x_{i} \mathbf{1}\right)}{\lambda_{i l}}+\right. \\
\left.+\frac{2 x_{i} \mathbf{1}^{\top} \boldsymbol{\Omega}_{i}\left(\rho_{2}\right)^{-1} \mathbf{1}-2 \mathbf{1}^{\top} \Omega_{i}\left(\rho_{2}\right)^{-1} \boldsymbol{X}_{i}}{\kappa_{i}}\right\} \\
\frac{\partial \ell}{\partial \alpha_{l}}=\sum_{i \in I} \boldsymbol{q}_{i \alpha_{l}},
\end{gathered}
$$

sendo $\boldsymbol{q}_{i \alpha_{l}}=\frac{\partial \ell_{i}}{\partial \alpha_{l}}=\frac{1}{\lambda_{i l}}\left(\mathbf{1}^{\top} \boldsymbol{\Omega}_{i l}\left(\boldsymbol{\rho}_{1 l}\right)^{-1} \boldsymbol{Y}_{i l}-\alpha_{l} \mathbf{1}^{\top} \boldsymbol{\Omega}_{i l}\left(\rho_{1 l}\right)^{-1} \mathbf{1}-\beta_{l} x_{i} \mathbf{1}^{\top} \boldsymbol{\Omega}_{i l}\left(\boldsymbol{\rho}_{1 l}\right)^{-1} \mathbf{1}\right)$.

$$
\frac{\partial \ell}{\partial \beta_{l}}=\sum_{i \in I} \boldsymbol{q}_{i \beta_{l}}
$$

sendo $\boldsymbol{q}_{i \beta_{l}}=\frac{\partial \ell_{i}}{\partial \beta_{l}}=\frac{1}{\lambda_{i l}}\left(x_{i} \mathbf{1}^{\top} \boldsymbol{\Omega}_{i l}\left(\rho_{1 l}\right)^{-1} \boldsymbol{Y}_{i l}-x_{i} \alpha_{l} \mathbf{1}^{\top} \boldsymbol{\Omega}_{i l}\left(\boldsymbol{\rho}_{1 l}\right)^{-1} \mathbf{1}-\beta_{l} x_{i}^{2} \mathbf{1}^{\top} \boldsymbol{\Omega}_{i l}\left(\boldsymbol{\rho}_{1 l}\right)^{-1} \mathbf{1}\right)$.

Fazendo $\boldsymbol{e}_{i l}=\boldsymbol{Y}_{i l}-\alpha_{l} \mathbf{1}-\beta_{l} 1 x_{i}, \boldsymbol{u}_{i}=\boldsymbol{X}_{i}-\mathbf{1} x_{i}$ e derivando em relação a $\lambda_{i l}$ e $\kappa_{i}$ para todo $i=\{1, \ldots, n\}$ e $l=\{1, \ldots, r\}$, teremos

$$
\frac{\partial \ell}{\partial \lambda_{i l}}=\boldsymbol{q}_{i \lambda_{i l}}
$$

sendo $\boldsymbol{q}_{i \lambda_{i l}}=\frac{\partial \ell_{i}}{\partial \lambda_{i l}}=-\frac{m}{2 \lambda_{i l}}+\frac{1}{2}\left\{\frac{\boldsymbol{e}_{i l}^{\top} \Omega_{i l}\left(\rho_{1 l}\right)^{-1} \boldsymbol{e}_{i l}}{\lambda_{i l}^{2}}\right\} \mathrm{e}$

$$
\frac{\partial \ell}{\partial \kappa_{i}}=q_{i \kappa_{i}}
$$

sendo $\boldsymbol{q}_{i \kappa_{i}}=\frac{\partial \ell_{i}}{\partial \kappa_{i}}=-\frac{m}{2 \kappa_{i}}+\frac{1}{2}\left\{\frac{\boldsymbol{u}_{i}^{\top} \Omega_{i}\left(\boldsymbol{\rho}_{1}\right)^{-1} \boldsymbol{u}_{i}}{\kappa_{i}^{2}}\right\}$.

Derivando em relação a $\rho_{1 j l}$ e $\rho_{2 j}$ para todo $j=1, \ldots, p$ teremos

$$
\frac{\partial \ell}{\partial \rho_{1 j l}}=\sum_{i=1}^{n} \boldsymbol{q}_{i \rho_{1 j l}}
$$

sendo 


$$
\begin{gathered}
\boldsymbol{q}_{i \rho_{1 j l}}=-\frac{1}{2} \operatorname{tr}\left\{\Omega_{i l}\left(\rho_{1 l}\right)^{-1} \boldsymbol{J}_{1 j l}\right\}+\frac{1}{2 \lambda_{i l}} \operatorname{tr}\left\{\Omega_{i l}\left(\rho_{1 l}\right)^{-1} \boldsymbol{J}_{1 j l} \Omega_{i l}\left(\rho_{1 l}\right)^{-1} \boldsymbol{e}_{i l} \boldsymbol{e}_{i l}^{\top}\right\} . \\
\frac{\partial \ell}{\partial \rho_{2 j}}=\sum_{i=1}^{n} \boldsymbol{q}_{i \rho_{2 j}}, \\
\boldsymbol{q}_{i \rho_{2 j}}=-\frac{1}{2} \operatorname{tr}\left\{\Omega_{i}\left(\boldsymbol{\rho}_{2}\right)^{-1} \boldsymbol{J}_{2 j}\right\}+\frac{1}{2 \kappa_{i}} \operatorname{tr}\left\{\Omega_{i}\left(\boldsymbol{\rho}_{2}\right)^{-1} \boldsymbol{J}_{2 j} \Omega_{i}\left(\rho_{2}\right)^{-1} \boldsymbol{u}_{i} \boldsymbol{u}_{i}^{\top}\right\} .
\end{gathered}
$$

As matrizes $\boldsymbol{J}_{1 j l}$ e $\boldsymbol{J}_{2 j}$ são as matrizes de derivadas de $\Omega_{i l}\left(\boldsymbol{\rho}_{1 l}\right)$ em relação ao $j$-ésimo de $\rho_{1 l}$ e $\Omega_{i}\left(\rho_{2}\right)$ em relação ao $j$-ésimo de $\rho_{2}$. Igualando (A.17)-(A.23) encontramos os estimadores abaixo

$$
\begin{aligned}
& \hat{x}_{i}=\frac{\kappa_{i} \sum_{l \in L}\left[\frac{\beta_{l} \mathbf{1}^{\top} \Omega_{i l}\left(\rho_{1 l}\right)^{-1}\left(\boldsymbol{Y}_{i l}-\alpha_{l} \mathbf{1}\right)}{\lambda_{i l}}\right]+\mathbf{1}^{\top} \boldsymbol{\Omega}_{i}\left(\rho_{2}\right)^{-1} \boldsymbol{X}_{i}}{\kappa_{i} \sum_{l \in L}\left[\frac{\beta_{l}^{2} \mathbf{1}^{\top} \Omega_{i l}\left(\rho_{1 l}\right)^{-1} \mathbf{1}}{\lambda_{i l}}\right]+\mathbf{1}^{\top} \Omega_{i}\left(\rho_{2}\right)^{-1} \mathbf{1}}, \\
& \hat{\beta}_{l}=\frac{\sum_{i \in I} \boldsymbol{\omega}_{i l} \hat{x}_{i}\left(\boldsymbol{Y}_{i l}-\bar{Y} \boldsymbol{\omega}_{l} \mathbf{1}\right)}{\sum_{i \in I} \boldsymbol{\omega}_{i l} \hat{x}_{i}\left(\hat{x}_{i}-\hat{\bar{x}}_{\left.\boldsymbol{\omega}_{l}\right) \mathbf{1}}\right.} \text { e } \hat{\alpha}_{l}=\bar{Y} \boldsymbol{\omega}_{l}-\hat{\beta} \hat{\bar{x}}_{\boldsymbol{\omega}_{l}}
\end{aligned}
$$

sendo que

$$
\bar{Y}_{\boldsymbol{\omega}_{l}}=\sum_{i \in I} \boldsymbol{\omega}_{i l} \boldsymbol{Y}_{i l}, \hat{\bar{x}}_{\boldsymbol{\omega}_{i l}}=\sum_{i \in I} \hat{x}_{i} \boldsymbol{\omega}_{i l} \mathbf{1}
$$

e

$$
\omega_{i l}=\frac{1^{\top} \Omega_{i l}\left(\rho_{1 l}\right)^{-1}}{\lambda_{i l} \sum_{i \in I}\left(1^{\top} \Omega_{i l}\left(\rho_{1 l}\right)^{-1} 1 / \lambda_{i l}\right)} .
$$

Os estimadores para $\lambda_{i l}$ e $\kappa_{i}$ são dados por

$$
\hat{\lambda}_{i l}=\frac{\hat{e}_{i l}^{\top} \Omega_{i l}\left(\rho_{1 l}\right)^{-1} \hat{\boldsymbol{e}}_{i l}}{m} \text { e } \hat{\kappa}_{i}=\frac{\hat{\boldsymbol{u}}_{i}^{\top} \boldsymbol{\Omega}_{i}\left(\rho_{2}\right)^{-1} \hat{\boldsymbol{u}}_{i}}{m}
$$


sendo $\hat{\boldsymbol{e}}_{i l}=\boldsymbol{Y}_{i l}-\left(\hat{\alpha}_{l}+\hat{\beta}_{l} \hat{x}_{i}\right) \mathbf{1}$ e $\hat{\boldsymbol{u}}_{i}=\boldsymbol{X}_{i}-\hat{x}_{i} \mathbf{1}$. Os estimadores para $\boldsymbol{\rho}_{1 l}$ e $\boldsymbol{\rho}_{2}$ são obtidos de forma iterativa, usando o algoritmo Escore de Fisher dado por

$$
\begin{aligned}
& \hat{\boldsymbol{\rho}}_{1 l}^{(v+1)}=\hat{\boldsymbol{\rho}}_{1 l}^{(v)}+\left.\mathrm{E}\left[\boldsymbol{I}\left(\hat{\boldsymbol{\rho}}_{1 l}^{(v)}\right)\right]^{-1} \frac{\partial \ell}{\partial \rho_{1 l}}\right|_{\rho_{1 l}=\hat{\boldsymbol{\rho}}_{1 l}^{(v)}} \\
& \hat{\boldsymbol{\rho}}_{2}^{(v+1)}=\hat{\boldsymbol{\rho}}_{2}^{(v)}+\left.\mathrm{E}\left[\boldsymbol{I}\left(\hat{\boldsymbol{\rho}}_{2}^{(v)}\right)\right]^{-1} \frac{\partial \ell}{\partial \rho_{2}}\right|_{\rho_{2}=\hat{\boldsymbol{\rho}}_{2}^{(v)}}
\end{aligned}
$$

onde os elementos de $\mathrm{E}\left[\boldsymbol{I}\left(\hat{\boldsymbol{\rho}}_{1 l}^{(v)}\right)\right]$ e $\mathrm{E}\left[\boldsymbol{I}\left(\hat{\boldsymbol{\rho}}_{2}^{(v)}\right)\right]$ são dados em (A.24) - (A.27). Os estimadores dos parâmetros $x_{i}, \alpha, \beta, \lambda_{i}$ e $\kappa_{i}$ no caso em que temos independências das réplicas em cada método de medição são dados fazendo $\Omega_{i l}\left(\rho_{1 l}\right)=\Omega_{i}\left(\rho_{2}\right)=I$ para todo $l=1,2, \ldots, r$.

$$
\begin{gathered}
\hat{x}_{i}=\frac{\kappa_{i} \sum_{l \in L}\left[\frac{\beta_{l}\left(\bar{Y}_{i l}-\alpha_{l}\right)}{\lambda_{i l}}\right]+\bar{X}_{i}}{\kappa_{i} \sum_{l \in L}\left[\frac{\beta_{l}^{2}}{\lambda_{i l}}\right]+1}, \\
\hat{\beta}_{l}=\frac{\sum_{i \in I} \omega_{i l} \hat{x}_{i}\left(\bar{Y}_{i l}-\bar{Y}_{\omega_{l}}\right)}{\sum_{i \in I} \omega_{i l} \hat{x}_{i}\left(\hat{x}_{i}-\hat{\bar{x}}_{\omega_{l}}\right) \mathbf{1}} \text { e } \hat{\alpha}_{l}=\bar{Y}_{\omega_{l}}-\hat{\beta} \hat{\bar{x}}_{\omega_{l}}
\end{gathered}
$$

sendo que

$$
\bar{Y}_{\omega_{l}}=\sum_{i \in I} \omega_{i l} \bar{Y}_{i l}, \hat{\bar{x}}_{\omega_{i l}}=\sum_{i \in I} \hat{x}_{i} \omega_{i l}, \omega_{i l}=\frac{1}{\lambda_{i l} \sum_{i \in I}\left(1 / \lambda_{i l}\right)} .
$$

e

$$
\hat{\lambda}_{i l}=\frac{\hat{e}_{i l}^{\top} \hat{e}_{i l}}{m} \text { e } \hat{\kappa}_{i}=\frac{\hat{u}_{i}^{\top} \hat{u}_{i}}{m}
$$

As derivadas segundas são dadas logo abaixo

$$
\frac{\partial^{2} \ell}{\partial \alpha_{l}^{2}}=-\sum_{i \in I}\left(1^{\top} \Omega_{i l}\left(\rho_{1 l}\right)^{-1} 1 / \lambda_{i l}\right) \Rightarrow \mathrm{E}\left(-\frac{\partial^{2} \ell}{\partial \alpha_{l}^{2}}\right)=\sum_{i \in I}\left(1^{\top} \Omega_{i l}\left(\rho_{1 l}\right)^{-1} 1 / \lambda_{i l}\right)
$$




$$
\begin{aligned}
& \frac{\partial^{2} \ell}{\partial \alpha_{l} \partial \beta_{l}}=-\sum_{i \in I}\left(\mathbf{1}^{\top} \boldsymbol{\Omega}_{i l}\left(\rho_{1 l}\right)^{-1} \mathbf{1} x_{i} / \lambda_{i l}\right) \Rightarrow \mathrm{E}\left(-\frac{\partial^{2} \ell}{\partial \alpha_{l} \partial \beta_{l}}\right)=\sum_{i \in I}\left(\mathbf{1}^{\top} \boldsymbol{\Omega}_{i l}\left(\rho_{1 l}\right)^{-1} \mathbf{1} x_{i} / \lambda_{i l}\right) \\
& \frac{\partial^{2} \ell}{\partial \alpha_{l} \partial \lambda_{i l}}=-\left(1 / \lambda_{i l}^{2}\right) 1^{\top} \Omega_{i l}\left(\rho_{1 l}\right)^{-1}\left(\boldsymbol{Y}_{i l}-\alpha_{l} \mathbf{1}-\beta_{l} \mathbf{1} x_{i}\right) \Rightarrow \mathrm{E}\left(-\frac{\partial^{2} \ell}{\partial \alpha_{l} \partial \lambda_{i l}}\right)=0 \\
& \frac{\partial^{2} \ell}{\partial \alpha_{l} \partial \kappa_{i}}=0 \Rightarrow \mathrm{E}\left(-\frac{\partial^{2} \ell}{\partial \alpha_{l} \partial \kappa_{i}}\right)=0 \\
& \frac{\partial^{2} \ell}{\partial \alpha_{l} \partial x_{i}}=\beta \mathbf{1}^{\top} \Omega_{i l}\left(\rho_{1 l}\right)^{-1} \mathbf{1} / \lambda_{i l} \Rightarrow \mathrm{E}\left(-\frac{\partial^{2} \ell}{\partial \alpha_{l} \partial x_{i}}\right)=\beta_{l} \mathbf{1}^{\top} \boldsymbol{\Omega}_{i l}\left(\rho_{1 l}\right)^{-1} \mathbf{1} / \lambda_{i l} \\
& \frac{\partial^{2} \ell}{\partial \alpha_{l} \partial \rho_{1 j l}}=-\sum_{i \in I} \frac{1}{\lambda_{i l}}\left\{\operatorname{tr}\left[\Omega_{i l}\left(\rho_{1 l}\right)^{-1} J_{1 j l} \Omega_{i l}\left(\rho_{1 l}\right)^{-1}\left(\boldsymbol{Y}_{i l} \mathbf{1}^{\top}-\alpha_{l} \mathbf{1} \mathbf{1}^{\top}-\beta_{l} x_{i} \mathbf{1} 1^{\top}\right)\right]\right\} \\
& \Rightarrow \mathrm{E}\left(-\frac{\partial^{2} \ell}{\partial \alpha_{l} \partial \rho_{1 l_{j}}}\right)=0 \\
& \frac{\partial^{2} \ell}{\partial \alpha_{l} \partial \rho_{2_{j}}}=0 \Rightarrow \mathrm{E}\left(-\frac{\partial^{2} \ell}{\partial \alpha_{l} \partial \rho_{2_{j}}}\right)=0 \\
& \frac{\partial^{2} \ell}{\partial \beta_{l}^{2}}=-\sum_{i \in I}\left(1^{\top} \Omega_{i l}\left(\rho_{1 l}\right)^{-1} 1 x_{i}^{2} / \lambda_{i l}\right) \Rightarrow \mathrm{E}\left(-\frac{\partial^{2} \ell}{\partial \beta_{l}^{2}}\right)=\sum_{i \in I}\left(1^{\top} \Omega_{i l}\left(\rho_{1 l}\right)^{-1} 1 x_{i}^{2} / \lambda_{i l}\right) \\
& \frac{\partial^{2} \ell}{\partial \beta_{l} \partial \lambda_{i l}}=-\left(1 / \lambda_{i l}^{2}\right) x_{i} \mathbf{1}^{\top} \boldsymbol{\Omega}_{i l}\left(\rho_{1 l}\right)^{-1}\left(\boldsymbol{Y}_{i l}-\alpha_{l} \mathbf{1}-\beta_{l} \mathbf{1} x_{i}\right) \Rightarrow \mathrm{E}\left(-\frac{\partial^{2} \ell}{\partial \beta_{l} \partial \lambda_{i l}}\right)=0 \\
& \frac{\partial^{2} \ell}{\partial \beta_{l} \partial \kappa_{i}}=0 \Rightarrow \mathrm{E}\left(-\frac{\partial^{2} \ell}{\partial \beta_{l} \partial \kappa_{i}}\right)=0 \\
& \frac{\partial^{2} \ell}{\partial \beta_{l} \partial x_{i}}=\frac{1}{\lambda_{i l}}\left(\mathbf{1}^{\top} \boldsymbol{\Omega}_{i l}\left(\rho_{1 l}\right)^{-1} \boldsymbol{Y}_{i l}-\alpha_{l} \mathbf{1}^{\top} \boldsymbol{\Omega}_{i l}\left(\rho_{1 l}\right)^{-1} \mathbf{1}-2 \beta_{l} x_{i} \mathbf{1}^{\top} \boldsymbol{\Omega}_{i l}\left(\rho_{1 l}\right)^{-1} \mathbf{1}\right)
\end{aligned}
$$




$$
\Rightarrow \mathrm{E}\left(-\frac{\partial^{2} \ell}{\partial \beta_{l} \partial \kappa_{i}}\right)=\frac{1}{\lambda_{i l}} \beta_{l} x_{i} \mathbf{1}^{\top} \boldsymbol{\Omega}_{i l}\left(\rho_{1 l}\right)^{-1} \mathbf{1}
$$

$$
\begin{aligned}
& \frac{\partial^{2} \ell}{\partial \beta_{l} \partial \rho_{1 j l}}=-\sum_{i \in I} \frac{x_{i}}{\lambda_{i l}}\left\{\operatorname{tr}\left[\Omega_{i l}\left(\rho_{1 l}\right)^{-1} J_{1 j l} \Omega_{i l}\left(\rho_{1 l}\right)^{-1}\left(\boldsymbol{Y}_{i l} \mathbf{1}^{\top}-\alpha_{l} \mathbf{1} \mathbf{1}^{\top}-\beta_{l} x_{i} \mathbf{1} \mathbf{1}^{\top}\right)\right]\right\} \\
& \Rightarrow \mathrm{E}\left(-\frac{\partial^{2} \ell}{\partial \beta_{l} \partial \rho_{1 j l}}\right)=0 \\
& \frac{\partial^{2} \ell}{\partial \beta_{l} \partial \rho_{2 j}}=0 \Rightarrow \mathrm{E}\left(-\frac{\partial^{2} \ell}{\partial \beta_{l} \partial \rho_{2 j}}\right)=0 \\
& \frac{\partial^{2} \ell}{\partial \lambda_{i l}^{2}}=-\left(1 / \lambda_{i l}^{3}\right) \boldsymbol{e}_{i l}^{\top} \boldsymbol{\Omega}_{i l}\left(\rho_{1 l}\right)^{-1} \boldsymbol{e}_{i l}+m /\left(2 \lambda_{i l}^{2}\right) \Rightarrow \mathrm{E}\left(-\frac{\partial^{2} \ell}{\partial \lambda_{i l}^{2}}\right)=m /\left(2 \lambda_{i l}^{2}\right) \\
& \frac{\partial^{2} \ell}{\partial \lambda_{i l} \partial \kappa_{i}}=0 \Rightarrow \mathrm{E}\left(-\frac{\partial^{2} \ell}{\partial \lambda_{i l} \partial \kappa_{i}}\right)=0 \\
& \frac{\partial^{2} \ell}{\partial \lambda_{i l} \partial x_{i}}=\frac{1}{2 \lambda_{i l}^{2}}\left[-2 \beta \mathbf{1}^{\top} \Omega_{i l}\left(\rho_{1 l}\right)^{-1}\left(\boldsymbol{Y}_{i l}-\alpha_{l} \mathbf{1}\right)+2 \beta_{l}^{2} x_{i} \mathbf{1}^{\top} \boldsymbol{\Omega}_{i l}\left(\rho_{1 l}\right)^{-1} \mathbf{1}\right] \\
& \Rightarrow E\left(-\frac{\partial^{2} \ell}{\partial \lambda_{i l} \partial x_{i}}\right)=0 \\
& \frac{\partial^{2} \ell}{\partial \lambda_{i l} \partial \rho_{1 j l}}=-\frac{1}{2 \lambda_{i l}^{2}} \operatorname{tr}\left[\Omega_{i l}\left(\rho_{1 l}\right)^{-1} \boldsymbol{J}_{1 j l} \Omega_{i l}\left(\boldsymbol{\rho}_{1 l}\right)^{-1} \boldsymbol{e}_{i l} \boldsymbol{e}_{i l}^{\top}\right] \Rightarrow \mathrm{E}\left(-\frac{\partial^{2} \ell}{\partial \lambda_{i l} \partial \rho_{1 j l}}\right)=\frac{1}{2 \lambda_{i l}} \operatorname{tr}\left[\Omega_{i l}\left(\rho_{1 l}\right)^{-1} \boldsymbol{J}_{1 j l}\right] \\
& \frac{\partial^{2} \ell}{\partial \lambda_{i l} \partial \rho_{2 j}}=0 \Rightarrow \mathrm{E}\left(-\frac{\partial^{2} \ell}{\partial \lambda_{i l} \partial \rho_{2 j}}\right)=0
\end{aligned}
$$

$$
\begin{gathered}
\frac{\partial^{2} \ell}{\partial \kappa_{i}^{2}}=-\left(1 / \kappa_{i}^{3}\right) \boldsymbol{u}_{i}^{\top} \boldsymbol{\Omega}_{i}\left(\boldsymbol{\rho}_{2}\right)^{-1} \boldsymbol{u}_{i}+m /\left(2 \kappa_{i}^{2}\right) \Rightarrow \mathrm{E}\left(-\frac{\partial^{2} \ell}{\partial \kappa_{i}^{2}}\right)=m /\left(2 \kappa_{i}^{2}\right) \\
\frac{\partial^{2} \ell}{\partial \kappa_{i} \partial x_{i}}=\frac{1}{2 \kappa_{i}^{2}}\left[2 x_{i} 1^{\top} \Omega_{i}\left(\rho_{2}\right)^{-1} \mathbf{1}-21^{\top} \boldsymbol{\Omega}_{i}\left(\boldsymbol{\rho}_{2}\right)^{-1} \boldsymbol{X}_{i}\right] \\
\Rightarrow \mathrm{E}\left(-\frac{\partial^{2} \ell}{\partial \kappa_{i} \partial x_{i}}\right)=0
\end{gathered}
$$




$$
\begin{aligned}
& \frac{\partial^{2} \ell}{\partial \kappa_{i} \partial \rho_{1 j l}}=0 \Rightarrow \mathrm{E}\left(-\frac{\partial^{2} \ell}{\partial \kappa_{i} \partial \rho_{1 j l}}\right)=0 \\
& \frac{\partial^{2} \ell}{\partial \kappa_{i} \partial \rho_{2 j}}=-\frac{1}{2 \kappa_{i}^{2}} \operatorname{tr}\left[\Omega_{i}\left(\rho_{2}\right)^{-1} \boldsymbol{J}_{2 j} \Omega_{i}\left(\boldsymbol{\rho}_{2}\right)^{-1} \boldsymbol{u}_{i} \boldsymbol{u}_{i}^{\top}\right] \Rightarrow \mathrm{E}\left(-\frac{\partial^{2} \ell}{\partial \kappa_{i} \partial \rho_{2 j}}\right)=\frac{1}{2 \kappa_{i}} \operatorname{tr}\left[\Omega_{i}\left(\boldsymbol{\rho}_{2}\right)^{-1} \boldsymbol{J}_{2 j}\right] \\
& \frac{\partial^{2} \ell}{\partial \rho_{1 j l}^{2}}=-\frac{1}{2} \sum_{i=1}^{n} \operatorname{tr}\left[\Omega_{i l}\left(\rho_{1 l}\right)^{-1} \boldsymbol{J}_{1 j l} \Omega_{i l}\left(\rho_{1 l}\right)^{-1} \boldsymbol{J}_{1 j l}\right] \\
& \Rightarrow \mathrm{E}\left(-\frac{\partial^{2} \ell}{\partial \rho_{1 j l}^{2}}\right)=\frac{1}{2} \sum_{i=1}^{n} \operatorname{tr}\left[\Omega_{i l}\left(\rho_{1 l}\right)^{-1} J_{1 j l} \Omega_{i l}\left(\rho_{1 l}\right)^{-1} J_{1 j l}\right] \\
& \frac{\partial^{2} \ell}{\partial \rho_{1 j_{1} l} \partial \rho_{1 j_{2} l}}=-\frac{1}{2} \sum_{i=1}^{n} \operatorname{tr}\left[\Omega_{i l}\left(\rho_{1 l}\right)^{-1} \boldsymbol{J}_{1 j_{1} l} \Omega_{i l}\left(\rho_{1 l}\right)^{-1} \boldsymbol{J}_{1 j_{2} l}\right] \\
& \Rightarrow \mathrm{E}\left(-\frac{\partial^{2} \ell}{\partial \rho_{1 j_{1} l} \partial \rho_{1 j_{2} l}}\right)=\frac{1}{2} \sum_{i=1}^{n} \operatorname{tr}\left[\Omega_{i l}\left(\rho_{1 l}\right)^{-1} J_{1 j_{1} l} \Omega_{i l}\left(\rho_{1 l}\right)^{-1} J_{1 j_{2} l}\right] \\
& \frac{\partial^{2} \ell}{\partial \rho_{2 j}^{2}}=-\frac{1}{2} \sum_{i=1}^{n} \operatorname{tr}\left[\Omega_{i}\left(\rho_{2}\right)^{-1} J_{2 j} \Omega_{i}\left(\rho_{2}\right)^{-1} J_{2 j}\right] \\
& \Rightarrow \mathrm{E}\left(-\frac{\partial^{2} \ell}{\partial \rho_{2 j}^{2}}\right)=\frac{1}{2} \sum_{i=1}^{n} \operatorname{tr}\left[\Omega_{i}\left(\rho_{2}\right)^{-1} J_{2 j} \Omega_{i}\left(\rho_{2}\right)^{-1} J_{2 j}\right] \\
& \frac{\partial^{2} \ell}{\partial \rho_{2 j_{1}} \partial \rho_{2 j_{2}}}=-\frac{1}{2} \sum_{i=1}^{n} \operatorname{tr}\left[\Omega_{i}\left(\rho_{2}\right)^{-1} J_{2 j_{1}} \Omega_{i}\left(\rho_{2}\right)^{-1} J_{2 j_{2}}\right] \\
& \Rightarrow \mathrm{E}\left(-\frac{\partial^{2} \ell}{\partial \rho_{2_{j_{1}}} \partial \rho_{2_{j_{2}}}}\right)=\frac{1}{2} \sum_{i=1}^{n} \operatorname{tr}\left[\Omega_{i}\left(\rho_{2}\right)^{-1} \boldsymbol{J}_{2 j_{1}} \Omega_{i}\left(\rho_{2}\right)^{-1} \boldsymbol{J}_{2 j_{1}}\right] \\
& \frac{\partial^{2} \ell}{\partial x_{i}^{2}}=\sum_{l=1}^{r}-\frac{1}{2}\left[2 \beta_{l}^{2} 1^{\top} \Omega_{i l}\left(\rho_{1 l}\right)^{-1} 1 / \lambda_{i l}+21^{\top} \Omega_{i}\left(\rho_{2}\right)^{-1} 1\right] \\
& \Rightarrow \mathrm{E}\left(-\frac{\partial^{2} \ell}{\partial x_{i}^{2}}\right)=\sum_{l=1}^{r} \frac{\beta_{l}^{2} \kappa_{i} \mathbf{1}^{\top} \Omega_{i l}\left(\rho_{1 l}\right)^{-1} \mathbf{1}+\lambda_{i l} \mathbf{1}^{\top} \Omega_{i}\left(\rho_{2}\right)^{-1} \mathbf{1}}{\lambda_{i l} \kappa_{i}}
\end{aligned}
$$


Para encontrar a matriz de covariâncias assintótica de $(\hat{\boldsymbol{\alpha}}, \hat{\boldsymbol{\beta}})$, utilizamos o mesmo procedimento adotado no caso de dois métodos. A inversa da matriz de covariâncias do vetor $\hat{\boldsymbol{\theta}}$ é dada por

$$
\Pi=\left[\begin{array}{lll}
\boldsymbol{A}^{*} & \boldsymbol{0}_{2 r \times(r+1)(n+p)} & \boldsymbol{B}^{*} \\
\boldsymbol{0}_{(r+1)(n+p) \times 2 r} & \boldsymbol{C}^{*} & \boldsymbol{0}_{(r+1)(n+p) \times n} \\
\boldsymbol{B}^{* \top} & \boldsymbol{0}_{n \times(r+1)(n+p)} & \boldsymbol{D}^{*}
\end{array}\right]
$$

sendo

$$
A^{*}=\left[\begin{array}{cc}
\boldsymbol{A}^{*}{ }_{11} & \boldsymbol{A}^{*}{ }_{12} \\
\boldsymbol{A}^{*}{ }_{12}^{\mathrm{T}} & \boldsymbol{A}^{*}{ }_{22}
\end{array}\right]
$$

com

$$
\begin{gathered}
\boldsymbol{A}^{*}{ }_{11}=\sum_{i \in I} \operatorname{diag}\left(\frac{\mathbf{1}^{\top} \Omega_{i 1}\left(\rho_{11}\right) \mathbf{1}}{\lambda_{i 1}}, \ldots \frac{1^{\top} \Omega_{i r}\left(\rho_{1 r}\right) \mathbf{1}}{\lambda_{i r}}\right) \\
A^{*}{ }_{12}=\sum_{i \in I} \operatorname{diag}\left(\frac{x_{i} \mathbf{1}^{\top} \Omega_{i 1}\left(\rho_{11}\right) \mathbf{1}}{\lambda_{i 1}}, \ldots \frac{x_{i} \mathbf{1}^{\top} \Omega_{i r}\left(\rho_{1 r}\right) \mathbf{1}}{\lambda_{i r}}\right) \\
A^{*}{ }_{22}=\sum_{i \in I} \operatorname{diag}\left(\frac{x_{i}^{2} \mathbf{1}^{\top} \Omega_{i 1}\left(\rho_{11}\right) \mathbf{1}}{\lambda_{i 1}}, \ldots \frac{x_{i}^{2} 1^{\top} \Omega_{i r}\left(\rho_{1 r}\right) \mathbf{1}}{\lambda_{i r}}\right) \\
B^{* \top}=\left[\begin{array}{ll}
B^{* \top}{ }_{1} & B^{* \top}{ }_{2}^{\top}
\end{array}\right]
\end{gathered}
$$

com

$$
B_{1}^{* \top}=\left[\begin{array}{ccc}
\beta_{1} \frac{1^{\top} \Omega_{i 1}\left(\rho_{11}\right)^{-1} \mathbf{1}}{\lambda_{11}} & \ldots & \beta_{r} \frac{\mathbf{1}^{\top} \Omega_{i r}\left(\rho_{1 r}\right)^{-1} \mathbf{1}}{\lambda_{1 r}} \\
\beta_{1} \frac{\mathbf{1}^{\top} \Omega_{i 1}\left(\rho_{11}\right)^{-1} 1}{\lambda_{21}} & \ldots & \beta_{r} \frac{\mathbf{1}^{\top} \Omega_{i r}\left(\rho_{1 r}\right)^{-1} \mathbf{1}}{\lambda_{2 r}} \\
\vdots & \vdots & \vdots \\
\beta_{1} \frac{\mathbf{1}^{\top} \Omega_{i 1}\left(\rho_{11}\right)^{-1} \mathbf{1}}{\lambda_{n 1}} & \ldots & \beta_{r} \frac{\mathbf{1}^{\top} \Omega_{i r}\left(\rho_{1 r}\right)^{-1} \mathbf{1}}{\lambda_{n r}}
\end{array}\right]
$$

e 
E finalmente

$$
\boldsymbol{B}_{2}^{* \top}=\left[\begin{array}{ccc}
\beta_{1} \frac{x_{1} \mathbf{1}^{\top} \Omega\left({ }_{i 1} \rho_{11}\right)^{-1} \mathbf{1}}{\lambda_{11}} & \ldots & \beta_{r} \frac{x_{1} \mathbf{1}^{\top} \Omega_{i r}\left(\rho_{1 r}\right)^{-1} \mathbf{1}}{\lambda_{1 r}} \\
\beta_{1} \frac{x_{2} \mathbf{1}^{\top} \Omega_{i 1}\left(\rho_{11}\right)^{-1} \mathbf{1}}{\lambda_{21}} & \ldots & \beta_{r} \frac{x_{2} \mathbf{1}^{\top} \Omega_{i r}\left(\rho_{1 r}\right)^{-1} \mathbf{1}}{\lambda_{2 r}} \\
\vdots & \vdots & \vdots \\
\beta_{1} \frac{x_{n} \mathbf{1}^{\top} \boldsymbol{\Omega}_{i 1}\left(\rho_{11}\right)^{-1} \mathbf{1}}{\lambda_{n 1}} & \ldots & \beta_{r} \frac{x_{n} \mathbf{1}^{\top} \Omega_{i r}\left(\rho_{1 r}\right)^{-1} \mathbf{1}}{\lambda_{n r}}
\end{array}\right]
$$

$$
\boldsymbol{D}^{*}=\sum_{l=1}^{r} \operatorname{diag}\left(\frac{\beta_{l}^{2} \kappa_{1} \mathbf{1}^{\top} \Omega_{i l}\left(\rho_{1 l}\right)^{-1} \mathbf{1}+\lambda_{1 l} \mathbf{1}^{\top} \boldsymbol{\Omega}_{i}\left(\rho_{2}\right)^{-1} \mathbf{1}}{\lambda_{1 l} \kappa_{1}}, \ldots, \frac{\beta_{l}^{2} \kappa_{n} \mathbf{1}^{\top} \Omega_{i l}\left(\rho_{1 l}\right)^{-1} \mathbf{1}+\lambda_{n l} \mathbf{1}^{\top} \Omega_{i}\left(\rho_{2}\right)^{-1} \mathbf{1}}{\lambda_{n l} \kappa_{n}}\right)
$$

Assim particionamos a matriz $\Pi$ da mesma forma que no caso anterior teremos

$$
\Pi^{-1}=\left[\begin{array}{ll}
\Pi_{11} & \Pi_{12} \\
\Pi_{12}^{\top} & \Pi_{22}
\end{array}\right]^{-1}=\left[\begin{array}{ll}
\Pi_{11}^{*} & \Pi_{12}^{*} \\
\Pi_{12}^{* \top} & \Pi_{22}^{*}
\end{array}\right]
$$

sendo

$$
\Pi_{11}=\left[\begin{array}{ll}
A^{*} & 0_{2 r \times(r+1)(n+p)} \\
0_{(r+1)(n+p) \times 2 r} & C^{*}
\end{array}\right] \quad, \quad \Pi_{12}^{\top}=\left[\begin{array}{ll}
B^{* \top} & 0_{n \times(r+1)(n+p)}
\end{array}\right] \quad \text { e } \quad \Pi_{22}=D^{*}
$$

Da mesma forma que no caso anterior estamos interessandos apenas em $\Pi_{11}^{*}$, e sabemos que

$$
\Pi_{11}^{*}=\left[\Pi_{11}-\Pi_{12} \Pi_{22}^{-1} \Pi_{12}^{\top}\right]^{-1}
$$

Assim substituindo teremos

$$
\Pi_{11}^{*}=\left[\begin{array}{ll}
A^{*}-B^{*} D^{*-1} B^{* \top} & 0_{2 r \times(r+1)(n+p)} \\
0_{(r+1)(n+p) \times 2 r} & C^{*}
\end{array}\right]^{-1}
$$

Portanto a matriz de covariâncias de $(\hat{\boldsymbol{\alpha}}, \hat{\boldsymbol{\beta}})$ é 


$$
\begin{aligned}
\operatorname{Cov}(\hat{\boldsymbol{\alpha}}, \hat{\boldsymbol{\beta}}) & =\left[\boldsymbol{A}^{*}-\boldsymbol{B}^{*} \boldsymbol{D}^{*-1} \boldsymbol{B}^{* \top}\right]^{-1} \\
& =\left[\begin{array}{cc}
\sum_{i \in I}\left\{\boldsymbol{F}_{i}-\frac{\boldsymbol{D}_{i} \boldsymbol{D}_{i}^{\top}}{a_{i}}\right\} & \sum_{i \in I} x_{i}\left\{\boldsymbol{F}_{i}-\frac{\boldsymbol{D}_{i} \boldsymbol{D}_{i}^{\top}}{a_{i}}\right\} \\
\cdot & \sum_{i \in I} x_{i}^{2}\left\{\boldsymbol{F}_{i}-\frac{\boldsymbol{D}_{i} \boldsymbol{D}_{i}^{\top}}{a_{i}}\right\}
\end{array}\right]^{-1}
\end{aligned}
$$

sendo

$$
\begin{gathered}
\boldsymbol{D}_{i}^{\top}=\left[\frac{\beta_{1}}{\lambda_{i 1}}\left(\mathbf{1}^{\top} \boldsymbol{\Omega}_{i 1}\left(\rho_{11}\right)^{-1} \mathbf{1}\right), \ldots, \frac{\beta_{r}}{\lambda_{i r}}\left(\mathbf{1}^{\top} \Omega_{i r}\left(\rho_{1 r}\right)^{-1} \mathbf{1}\right)\right], \\
\boldsymbol{F}_{i}=\operatorname{diag}\left\{\frac{\beta_{1}}{\lambda_{i 1}}\left(\mathbf{1}^{\top} \boldsymbol{\Omega}_{i 1}\left(\rho_{11}\right)^{-1} \mathbf{1}\right), \ldots, \frac{\beta_{r}}{\lambda_{i r}}\left(\mathbf{1}^{\top} \Omega_{i r}\left(\rho_{1 r}\right)^{-1} \mathbf{1}\right)\right\}
\end{gathered}
$$

e

$$
a_{i}=\sum_{q=1}^{r} \frac{\beta_{q}^{2} \kappa_{i} \mathbf{1}^{\top} \boldsymbol{\Omega}_{i q}\left(\rho_{1 q}\right)^{-1} \mathbf{1}+\lambda_{i q} \mathbf{1}^{\top} \boldsymbol{\Omega}_{i}\left(\rho_{2}\right)^{-1} \mathbf{1}}{\lambda_{i q} \kappa_{i}}
$$

e para o caso em que as réplicas são independentes teremos

$$
\begin{gathered}
\boldsymbol{D}_{i}^{\top}=m\left[\frac{\beta_{1}}{\lambda_{i 1}}, \ldots, \frac{\beta_{r}}{\lambda_{i r}}\right], \\
\boldsymbol{F}_{i}=m \operatorname{diag}\left\{\frac{\beta_{1}}{\lambda_{i 1}}, \ldots, \frac{\beta_{r}}{\lambda_{i r}}\right\}
\end{gathered}
$$

e

$$
a_{i}=m \sum_{q=1}^{r} \frac{\beta_{q}^{2} \kappa_{i}+\lambda_{i q}}{\lambda_{i q} \kappa_{i}} .
$$




\section{Referências Bibliográficas}

Adcock, R. J. (1878). The problem in least squares. The Analyst, v. 5, p. 53.

Barnett, R. N. (1965). A scheme for the comparison of quantitative methods. The American Journal of Clinical Pathology, v. 43, p. 562-569.

Barnett, V. D. (1970). Fitting straight lines-the linear functional relationship with replicated observations. Applied Statistics, v. 19, p. 135-144.

Castilho, M. V. (2004). A comparison os statistical techniques for detecting analytical bias in geoanalysis. Geostandards and Geoanalytical research, v. 28, p. 277-290.

Castro, M.; Castilho, M. V. and Bolfarine, H. (2004). Consistent estimation and testing in comparing analytical bias models. [S.1.].

Castro, M. et al. (2005). Detection of analytical bias when comparing two or more measuring methods. Journal of Chemometrics, v. 19, p. 1-10.

Cheng, C. L. and Van Ness, J. W. (1991). On the unreplicated ultrastructural model. Biometrika, v. 78 , p. 442-445.

Dolby, G. R.; Cormack, R. M. and Sinclair, D. F. (1987). On fitting bivariate functional relationships to unpaired and unequally replicated data. Biometrika, v. 74, p. $393-399$. 
Dolby, G. R. and Lipton, S. (1972). Maximum likelihood estimation of the general nonlinear functional relationship with replicated observations and correlated erros. Biometrika, v. 59, p. 121-129.

Fuller, W. A. (1987). Measurement Error Models. New York, USA: John Wiley. Galea-Rojas, M. et al. (2003). Detection of analytical bias. Analyst, v. 128, p. 1073-1081.

Galea-Rojas, M. et al. (2005). On analytical bias methods using errors-in-variables models.

Gimenez, P. and Bolfarine, H. (1997). Corrected score functions in classical errors-in-variables and incidental parameter models. The Australian Journal of Statistics, v. 39, p. 325-344.

Kimura, D. (1992). Functional comparative calibration using an em algorithm. Biometrics, v. 48, p. 1263-1271.

Lehmann, E. L. (1998). Elements of Large-Sample Theory. New York, USA: Springer.

Mak, T. K. (1982). Estimation in the presence of incidental parameters. Canadian Journal Statistics, v. 10, p. 121.

Patefield, W. M. (1977). On the information matrix in the linear function relationship problem. Applied Statistics, v. 26, p. 69-70.

Patefield, W. M. (1978). The unreplicated ultrastructural relation: Large sample properties. Biometrika, v. 65 , p. 535-540.

R Development Core Team. (2006). R: A Language and Environment for Statistical Computing. Vienna, Austria. ISBN 3-900051-07-0. Disponível em: $<$ http://www.R-project.org > 
Ripley, B. D. and Thompson, M. (1987). Regression techniques for the detection of analytical bias. Analyst, v. 112, p. 377-383.

Riu, J. and Rius, X. F. (1996). Assenssing the accuracy of analytical methods using linear regression with errors in both axes. Analytical Chemistry, v. 68, p. 1851-1857.

Stefanski, L. A. (2000). Measurement error models. Journal of the American Statistical Association, v. 95, p. 1353-1358.

Taylor, J. (1973). A method of fitting several linear functinal relationship and of testing of differences between them. Applied Statistics, v. 22, p. 239-248.

Westgard, J. O. and Hunt, M. R. (1973). Use and interpretation of common statistical tests in method-comparison studies. Clinical Chemistry, v. 19, p. 49-57.

Williamson, J. (1968). Least squares fitting of a straight line. Canadian Journal of Physics, v. 46, p. 1845-1847.

York, D. (1966). Least squares fitting of a straight line. Canadian Journal of Physics, v. 44, p. 1079-1086. 